\title{
Monte Carlo dose calculations for breast and lung permanent implant brachytherapy
}

\author{
by \\ Justin G. H. Sutherland \\ A thesis submitted to the Faculty of Graduate and Postdoctoral Affairs \\ in partial fulfillment of the requirements for the degree of
}

\section{Doctor of Philosophy}

in

\author{
Physics \\ Specialization in Medical Physics \\ Ottawa-Carleton Institute for Physics \\ Department of Physics \\ Carleton University \\ Ottawa, Ontario, Canada
}

September 2013

(C) 2013 Justin G. H. Sutherland 


\section{Abstract}

Current clinical practice for dose calculations for brachytherapy utilizes the TG43 formalism where absorbed dose is calculated in a homogeneous water environment. The formalism does not account for the effect of tissue heterogeneities, interseed attenuation, and the finite dimensions of patients causing significant errors in calculated doses for low-energy permanent implant brachytherapy. As an alternative, Monte Carlo (MC) dose calculations model radiation transport and dose deposition in nonwater media but have only seen recent application to brachytherapy; issues relating to accurate MC calculations for permanent brachytherapy are unresolved and, in some cases, are completely unexplored. This thesis investigates the accurate application of MC dose calculations for permanent implant breast and lung brachytherapy.

MC calculations of permanent implant breast brachytherapy have commonly used a single tissue with a composition averaging that of fibroglandular and adipose tissues. Changes in dose due to segmenting gland and adipose tissues and calcifications are determined and results suggest that averaged tissues produce inaccurate photon energy fluences and thus, in most cases, are unsuitable for calculating doses.

The first investigation of patient-specific MC dose calculations for permanent implant ${ }^{125} \mathrm{I}$ lung brachytherapy is presented where the modelling choices that significantly affect doses are determined and deviations of up to $36 \%$ from TG-43 calculated doses are found. CT image artifact reduction and organ-constrained tissue assignment are studied to find improved techniques for modelling patients. MC calculations are used to determine that current lung brachytherapy treatment planning practices are insufficient for producing dose distributions in patients since implant deformation and patient tissues significantly change planning dose distributions. Finally, current and novel radionuclides for permanent implant lung brachytherapy are explored through a comprehensive study of doses to treatment volumes and critical organs. 


\section{Acknowledgments}

I would like to express my sincere gratitude towards my supervisors, Dr. Rowan Thomson and Dr. Dave Rogers. Together, they have provided me with immeasurable guidance, direction, and expertise. Dave sets a high standard of excellence that will surely affect me throughout my future endeavours. Additionally, his open-door policy and cheerful insights have always been greatly appreciated. Rowan's tireless involvement with the work of her students shows in her enthusiasm and willingness to always provide valuable guidance. The decision for Rowan to be my co-supervisor when she became a faculty member was easily one of the most advantageous events of my time at Carleton. I hope that she considers taking me on as her first student to be a fraction as positive a milestone as being her student has been for me.

I would like to thank Dr. Keith Furutani of the Mayo Clinic for his highly important insights and perspective on our collaborative work. The majority of this thesis would not have been possible without him. I would also like to thank a number of my colleagues at Carleton including Bryan Muir, Nelson Miksys, Elsayed Ali, Marielle Lesperance, Martin Martinov, and Manuel Rodriguez for their help and friendship.

I'm always deeply grateful to my parents for their love and support. Their encouragement and enthusiasm for my achievemen ts have been invaluable. To my wife, Becky: thank you for your support, love, encouragement, and most importantly your patience! You've always been there for me and I can't thank you enough for the irreplaceable role you've played in my life. Finally, I want to thank my one-year-old son, Owen, for the endless joy he's added to our lives.

This work has been supported by an Ontario Graduate Scholarship in science

and technology, an Ontario Graduate Scholarship, as well as the Kiwanis Club of Ottawa Medical Foundation and Dr. Kanta Marwah Scholarship in Medical Physics. 


\section{Statement of originality}

This thesis is a summary of the most significant portion of the author's work during the course of his Ph.D. program at Carleton University. It is based on the journal papers listed below. Most of the results have or will also be presented at national and international conferences.

Dr. Dave Rogers and Dr. Rowan Thomson supervised the project and provided input on all of its components, including the publications. Otherwise, the author of this thesis performed all of the computational work, and drafted and revised all of the manuscripts. A description of where the results of each publication can be found in the thesis is given below.

\section{Peer-reviewed papers}

I. J. G. H. Sutherland and D. W. O. Rogers, "Monte Carlo calculated absorbed-dose energy dependence of EBT and EBT2 film", Med. Phys. 37, 1110 - 1116 (2010).

- The results of this paper are unrelated to the topic of the thesis and are not included. The paper is listed to provide a complete view of the author's publications.

II. J. G. H. Sutherland, R. M. Thomson, and D. W. O. Rogers, "Changes in dose with segmentation of breast tissues in Monte Carlo calculations for low-energy brachytherapy", Med. Phys. 38, 4858 - 4865 (2011).

- The results of this paper appear in chapter 3 when discussing segmentation of breast tissues for Monte Carlo calculations of low-energy brachytherapy.

- The results of this paper were presented as a poster at the Joint AAPM/COMP Meeting in Vancouver, BC (2011). 
III. J. G. H. Sutherland, K. M. Furutani, Y. I. Garces, and R. M. Thomson, "Modelbased dose calculations for ${ }^{125}$ I lung brachytherapy", Med. Phys. 39, 4365 - 4377 (2012).

- The results of this paper appear in chapter 4 when discussing Monte Carlo calculations for ${ }^{125} \mathrm{I}$ lung brachytherapy.

- The results of this paper were presented as an oral presentation at the Joint AAPM/COMP Meeting in Vancouver, BC (2011).

IV. J. G. H. Sutherland, K. M. Furutani, and R. M. Thomson, "A Monte Carlo investigation of lung brachytherapy treatment planning", Phys. Med. Biol., 58, 4763 4780 (2013).

- The results of this paper appear in chapter 6 when discussing a Monte Carlo investigation of lung brachytherapy treatment planning.

- The results of this paper were presented as an oral presentation at the COMP Annual Scientific Meeting in Halifax, NS (2012).

V. J. G. H. Sutherland, N. Miksys, K. M. Furutani, and R. M. Thomson, "Metallic artifact mitigation and organ-constrained tissue assignment for Monte Carlo calculations of lung brachytherapy", Med. Phys., submitted.

- The results of this paper appear in chapter 5 when discussing the comparison of metallic artifact reduction techniques and organ-constrained tissue assignments for Monte Carlo of permanent implant lung brachytherapy.

- The results of this paper were presented as an oral presentation at the AAPM Annual Meeting \& Exhibition in Indianapolis, IN (2013).

VI. J. G. H. Sutherland, K. M. Furutani, and R. M. Thomson, "Monte Carlo calculated doses to treatment volumes and organs at risk for lung brachytherapy", Phys. Med. Biol., submitted.

- The results of this paper appear in chapter 7 when discussing the Monte Carlo calculated doses to treatment volumes and organs at risk using various radionuclides for permanent implant lung brachytherapy.

- The results of this paper will be presented as a poster at the CARO-COMP Joint Scientific Meeting in Montreal QC (2013). 


\section{Contents}

Abstract

Acknowledgements

Statement of originality iv

Contents vi vi v v v

List of Tables $\quad \mathrm{x}$

List of Figures xii

$\begin{array}{lll}1 & \text { Introduction } & 1\end{array}$

1.1 Brachytherapy . . . . . . . . . . . . . . . . . . . . . . . 2

1.1.1 Permanent breast seed implant brachytherapy (PBSI) . . . . . 2

1.1.2 Intraoperative brachytherapy for stage I non-small cell lung carcinoma ..................... . . 4

1.2 Current clinical brachytherapy dosimetry practice: TG-43 . . . . . . 5

1.3 Model-based dose calculations for brachytherapy . . . . . . . . . . . . . 7

1.4 Monte Carlo simulations . . . . . . . . . . . . . . . . . . . . . . . . . 8

1.5 Outline of thesis . . . . . . . . . . . . . . . . . . . 11

\begin{tabular}{|lll}
2 & Model-based dose calculations for brachytherapy & 13
\end{tabular} 


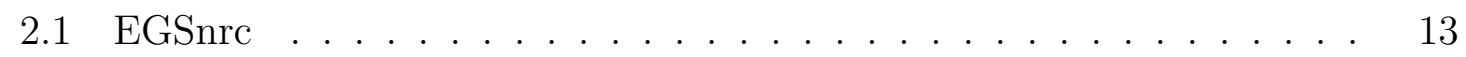

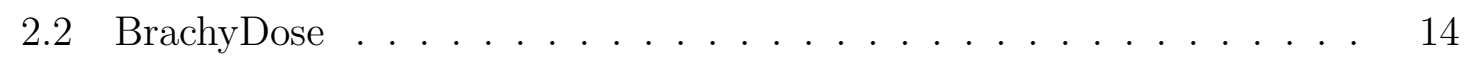

$2.3 \quad$ Calculating absolute dose $\ldots \ldots \ldots \ldots \ldots \ldots$

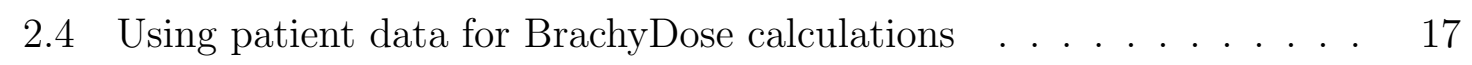

$2.4 .1 \quad$ Elemental composition of voxels $\ldots \ldots \ldots \ldots$

2.4 .2 Tools for files in DICOM format . . . . . . . . . . . . 18

2.5 Simulation parameters $\ldots \ldots \ldots \ldots \ldots \ldots$

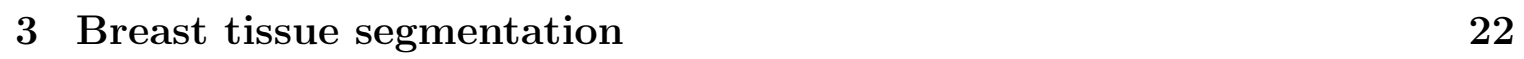

3.1 Methods . . . . . . . . . . . . . . . . . . . . . . . . . . . . . . . . . . . . 23

3.1.1 Dose to gland and adipose versus dose to an averaged tissue . 25

$3.1 .2 \quad$ Effect of realistic segmentation on photon energy fluence . . . . . . . . . . . . . . . . 27

3.1 .3 The effect of calcifications on photon energy fluence . . . . . 28

3.2 Results and Discussion . . . . . . . . . . . . . . . . . . . . . . . . . . . . . $\quad 29$

$3.2 .1 \quad$ Dose to gland and adipose versus dose to an averaged tissue . 29

$\begin{array}{lll}3.2 .2 & \text { Realistic segmentation and its effect on photon energy fluence } & 32\end{array}$

3.2 .3 The effect of calcifications $\ldots \ldots \ldots \ldots \ldots$

3.3 Conclusions $\ldots \ldots \ldots \ldots$

4 Exploring model-based dose calculations for ${ }^{125}$ I lung brachytherapy 43

4.1 Methods . . . . . . . . . . . . . . . . . . . . . . . . . 45

4.1 .1 Correcting metallic artifacts . . . . . . . . . . 46

4.1 .2 Tissue assignment schemes . . . . . . . . . . . . . 50

$4.1 .3 \quad$ Comparison with TG-43 $\ldots \ldots \ldots \ldots$

4.2 Results . . . . . . . . . . . . . . . . . . . . . . . . . . . . . 55 
$4.2 .1 \quad$ Correcting metallic artifacts . . . . . . . . . . . . . . 55

4.2 .2 Tissue assignment schemes . . . . . . . . . . . . . . . . . . . . 59

4.2 .3 Comparison with TG-43 . . . . . . . . . . . . . . . 62

4.3 Discussion . . . . . . . . . . . . . . . . . . . . . . . . 65

4.4 Conclusions $\ldots \ldots \ldots$. . . . . . . . . . . . . . . . . . . . . . . . . 70

5 Metallic artifact mitigation and organ-constrained tissue assignment $\begin{array}{ll}\text { for lung brachytherapy } & 72\end{array}$

5.1 Methods . . . . . . . . . . . . . . . . . . . . . . . . 74

$5.1 .1 \quad$ Metallic artifact reduction . . . . . . . . . . . . . . . . . 74

5.1 .2 Tissue assignment schemes and development of computational phantoms ....................... 75

5.2 Results and Discussion . . . . . . . . . . . . . . . . . . . . 78

5.2 .1 Metallic artifact reduction . . . . . . . . . . . . . . . 78

5.2 .2 Tissue assignment schemes and dose . . . . . . . . . . . . . 83

5.3 Conclusions . . . . . . . . . . . . . . . . . . . . . . . . 91

6 A Monte Carlo investigation of lung brachytherapy treatment planning $\quad 95$

6.1 Methods . . . . . . . . . . . . . . . . . . . 96

$6.1 .1 \quad$ Effect of non-water media on prescription dose . . . . . . . . . 97

6.1.2 Effect of seed grid deformation and patient-defined media. . . 99

6.2 Results and discussion . . . . . . . . . . . . . . . . . . . . 103

6.2 .1 Effect of non-water media on prescription dose . . . . . . . . . 103

6.2.2 Effect of seed grid deformation and patient-defined media . . . 109

6.3 Conclusions . . . . . . . . . . . . . . . . . . . . . . . 114 
7 Monte Carlo calculated doses to treatment volumes and organs at $\begin{array}{ll}\text { risk for permanent implant lung brachytherapy } & 117\end{array}$

7.1 Methods . . . . . . . . . . . . . . . . . . . . . 119

7.2 Results and Discussion . . . . . . . . . . . . . . . . . . . . . . . . . . 124

7.2 .1 Doses to treatment volumes . . . . . . . . . . . . . . . . . 124

7.2 .2 Doses to the right lung . . . . . . . . . . . . . . 130

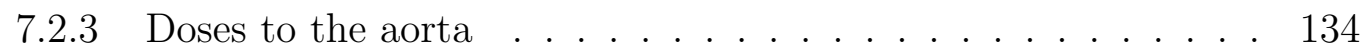

7.2 .4 Doses to the heart . . . . . . . . . . . . . . . . . . . . . . 138

7.3 Conclusions $\ldots \ldots \ldots$. . . . . . . . . . . . . . . . . . . . . . . . . 140

$\begin{array}{lll}8 & \text { Conclusions } & 142\end{array}$

8.1 Overview. . . . . . . . . . . . . . . . . . . . . . . . . . 142

8.2 Summary of conclusions . . . . . . . . . . . . . . . . . 143

8.3 Impact and future work . . . . . . . . . . . . . . . . . . 145

\begin{tabular}{ll}
\hline References & 147
\end{tabular} 


\section{List of Tables}

2.1 Composition of tissues used in calculations . . . . . . . . . . . . . 19

$4.1 \quad$ Mass attenuation and energy absorption coefficients for $28 \mathrm{keV}$ photons 51

4.2 CT number / mass density calibration data . . . . . . . . . . 52

4.3 Dose metrics of lung brachytherapy patients for artifact corr. methods 59

4.4 Dose metrics of lung brachytherapy patients for tissue assign. schemes 61

4.5 Dose metrics of lung brachytherapy patients using TG-43 and MBDCs 65

$5.1 \quad$ Summary of metallic artifact reduction techniques . . . . . . . . . . . 76

$5.2 \quad$ Dose metrics for various phantoms of lung brachytherapy patient 1 . 84

$5.3 \quad$ Dose metrics for various phantoms of lung brachytherapy patient $2 \quad$. $\quad 85$

$5.4 \quad$ Dose metrics for various phantoms of lung brachytherapy patient $5 \quad$. 86

$5.5 \quad$ Percentage of voxels assigned to bone for phantoms . . . . . . . . . . 87

6.1 Mass attenuation and energy absorption coefficients for 28 and $30 \mathrm{keV}$ photons ........................... 97

6.2 Lung brachytherapy treatment planning nomogram seed configurations 98

6.3 Notation and description of dose calculation for prescription dose analysis 99

6.4 Monte Carlo calculated nomograms for TG-43 $3_{\text {point }}$ and $\mathrm{D}_{1,1(0.26)}$. . . . 108

$6.5 \mathrm{~V}_{100}$ for PTV05 and PTV20 for virtual implants . . . . . . . . . . . . 111

$6.6 \quad \mathrm{D}_{90}$ for PTV05 and PTV20 for virtual implants . . . . . . . . . . . . 112 
7.1 Mass attenuation and energy absorption coefficient ratios of water to tissues . . . . . . . . . . . . . . . . . . 120

7.2 Mean and standard deviations of $\mathrm{D}_{90} \ldots \ldots \ldots \ldots$. . . . . . . . . 125

7.3 Mean and standard deviations of $\mathrm{V}_{100}$ and $\mathrm{V}_{200}$. . . . . . . . . . . . 128

7.4 Mean dose to lung for fissure implant . . . . . . . . . . . . . . . . . . 132 


\section{List of Figures}

1.1 Diagram of PBSI implant procedure . . . . . . . . . . . . . . 3

1.2 Diagram of lung brachytherapy procedure $\ldots \ldots \ldots \ldots$

1.3 Diagram of TG-43 coordinate system . . . . . . . . . . . . . 7

1.4 Mass attenuation and energy absorption ratios $\ldots \ldots \ldots \ldots$

$2.1 \quad$ Diagram depicting calculation of dose to a fictitious voxel medium . . 17

3.1 Diagrams of various breast phantoms $\ldots \ldots \ldots \ldots \ldots$

3.2 DVHs for dose to gland in averaged tissue and randomly segmented

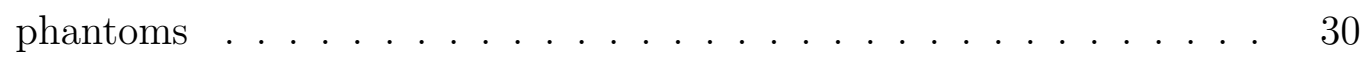

$3.3 \quad$ DVHs for entire phantoms in averaged tissue and randomly segmented

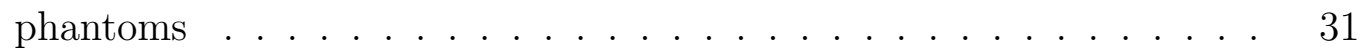

3.4 Dose profiles for various breast phantoms . . . . . . . . . . . . . . 32

$3.5 \quad$ DVHs for dose to various tissues in realistically segmented and averaged tissue phantoms . . . . . . . . . . . . . . . . . 33

$3.6 \quad$ DVHs for entire phantoms for realistically segmented and averaged

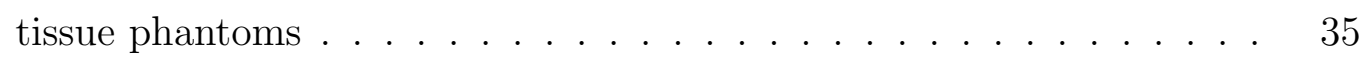

$3.7 \quad$ DVHs for dose to gland voxels in phantoms with calcification . . . . 38

3.8 DVHs for entire phantoms for phantoms with calcification . . . . . 39

3.9 DVHs for dose to non-calcification media for phantoms with calcification 41 
4.1 CT profiles for uncorrected and vsMAR corrected data for a lung brachytherapy patient . . . . . . . . . . . . . . . . . . . . 48

$4.2 \quad$ A portion of a CT image showing artifact reduction methods . . . . . 49

4.3 Histogram showing the frequency of CT numbers in several lung brachytherapy patient CT phantoms . . . . . . . . . . . . . . . . . . . 52

4.4 Tissue assignment schemes . . . . . . . . . . . . . . . . . . . 54

4.5 Dose profiles showing results of artifact reduction in a lung brachytherapy patient . . . . . . . . . . . . . . 56

4.6 CT image showing line used for profile in figure 4.5 . . . . . . . . . 56

4.7 DVHs showing result of metallic artifact reduction in a lung brachytherapy patient . . . . . . . . . . . . . . . . . . . 60

4.8 DVHs showing result of tissue assignment schemes in a lung brachytherapy patient . . . . . . . . . . . . . . . . . 62

4.9 Dose profile showing result of tissue assignment schemes in a lung brachytherapy patient ................ 63

4.10 CT image showing the line used for the dose profile in figure 4.9 . . . 63

4.11 DVHs comparing TG-43 and MBDCs for two lung brachytherapy patients 66

4.12 Dose profiles comparing TG-43 and MBDCs for a lung brachytherapy patient . . . . . . . . . . . . . . . . 67

$5.1 \quad$ Diagram showing constrained and unconstrained tissue assign. schemes 77

$5.2 \quad$ Uncorrected CT image for a lung brachytherapy patient. . . . . . . . 78

5.3 CT images and difference images after application of artifact reduction 79

$5.4 \quad$ CT number profiles for artifact reduced CT images . . . . . . . . . . 82

5.5 Dose profiles for phantoms of lung brachytherapy patient 2 . . . . . 89

$5.6 \quad$ Dose and density profiles illustrating effect of using nominal tissue densities . . . . . . . . . . . . . . . . . . . . 91 
5.7 Composite dose and density distributions showing profile lines for figure $5.6 \ldots \ldots \ldots . \ldots \ldots 2 \ldots \ldots . \ldots \ldots$

$6.1 \quad$ Diagram showing nomogram seed configurations B and D . . . . . . . 98

6.2 Illustration showing the 5 virtual implants and contours of right lung 100

6.3 Illustration of the virtual implant creation algorithm . . . . . . . . . 101

6.4 Normalized prescription doses for different dose calculations . . . . . 104

6.5 Isodose contours comparing TG- $43_{\text {sim }}$ to $\mathrm{D}_{1,1(0.26)}$ for planning seed configuration ........................ 106

6.6 Normalized $\mathrm{D}_{1,1(0.26)}$ prescription doses for different seed configurations 107

6.7 TG-43 $3_{\text {sim }}$ DVHs for PTV05s illustrating effects of deformation . . . . 109

6.8 DVHs for PTV05 of "fissure" virtual implant. . . . . . . . . . . . . . 112

6.9 DVHs for PTV05 and PTV20 of "superior" virtual implant . . . . . . 113

6.10 Dose and density profiles for "superior" virtual implant . . . . . . . . 115

$7.1 \quad$ Diagram of tissue assignment scheme for lung doses . . . . . . . . . . 121

$7.2 \quad$ CT image with heart and aorta contours and centres of implants . . . 122

7.3 Illustration of virtual implant no. 1 . . . . . . . . . . . . . . . . 123

$7.4 \quad \mathrm{D}_{90}$ for treatment volumes for source types . . . . . . . . . . . . . . 126

$7.5 \mathrm{~V}_{100}$ for treatment volumes for source types $\ldots$. . . . . . . . . . . . 129

7.6 Mean dose to lung for implants . . . . . . . . . . . . . . . . . . . . . 131

7.7 DVH of dose to lung for fissure implant . . . . . . . . . . . . . . . . . 133

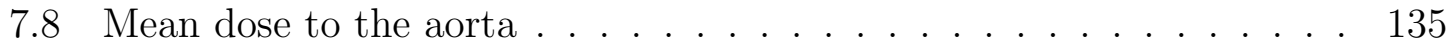

$7.9 \mathrm{~V}_{5}$ of the heart for implants $\ldots \ldots \ldots \ldots$. . . . . . . . . 137

7.10 Mean dose to the heart for implants . . . . . . . . . . . . . . . . . . . 139 


\section{Chapter 1}

\section{Introduction}

According to the Canadian Cancer Society, approximately $40 \%$ of Canadians will develop cancer in their lifetimes and $25 \%$ will die of the disease ${ }^{1}$. Common cancer treatments are surgery, chemotherapy, immunotherapy, monoclonal antibodies, and radiation therapy. Radiation therapy is the medical use of ionizing radiation to control malignant tumour cells. Often used in concert with surgery and/or chemotherapy both curatively and palliatively, the objective of radiation therapy is to destroy malignant cells by damaging their genetic material while simultaneously sparing as much healthy tissue as possible.

The three principal modes of radiation therapy are systemic radiation therapy, external beam radiation therapy, and brachytherapy ${ }^{2}$. Systemic radiation therapy targets cancer cells by injecting a liquid radiation source into the body. External beam radiation therapy directs radiation at a patient's body from an external machine. Common sources for external beam radiation therapy are $\gamma$ rays produced by radionuclides such as ${ }^{60} \mathrm{Co}$ and $\mathrm{x}$ rays, electrons, and heavier charged particles such as protons produced by linear accelerators (linacs) ${ }^{3}$. Brachytherapy, the focus of this thesis, is described in the following section. 


\subsection{Brachytherapy}

Brachytherapy is a form of radiation therapy where a radiation source is placed next to or inside a treatment site and is used as a treatment for cervical, prostate, skin, ocular, breast and lung cancers among other sites. Brachytherapy treatments are commonly divided into two forms (see eg. Podgorsak et al. ${ }^{4}$ ). In high-dose rate (HDR) brachytherapy, the rate of dose delivery is greater than $12 \mathrm{~Gy} \cdot \mathrm{h}^{-1}$ and single sources (commonly ${ }^{192} \mathrm{Ir}$ ) are delivered to the treatment site for a short period of time using catheters and a remote afterloader. Low-dose rate (LDR) treatments have numerous encapsulated sources or seeds (approximately the size of a grain of rice) that emit at a rate of $2 \mathrm{~Gy} \cdot \mathrm{h}^{-1}$ or less to a treatment-site-specific reference point and are surgically implanted either permanently or are removed after several days.

The use of low energy $(\lesssim 50 \mathrm{keV})$ radionuclides for permanent implant LDR brachytherapy began in the late 1960 s with ${ }^{125} \mathrm{I} \mathrm{seeds}^{5}$, followed by the adoption of ${ }^{103} \mathrm{Pd}$ and later ${ }^{131} \mathrm{Cs}$. The ease of manipulating and disposing of these seeds makes them attractive sources and their use has revolutionized the practice of permanent implantation. The most common use of these low energy seeds is permanent seed implantation for the treatment of early stage prostate carcinoma, but permanent seed implantation treatments have also been more recently developed as adjuvant radiation treatments for early stage breast cancer ${ }^{6 / 7}$ and stage I non-small cell lung cancer $^{[8] 9}$ which are of particular interest in this thesis.

\subsubsection{Permanent breast seed implant brachytherapy (PBSI)}

While breast cancer is the most commonly diagnosed cancer in women, the majority of women are diagnosed with early-stage, localized disease due to widespread screen- 


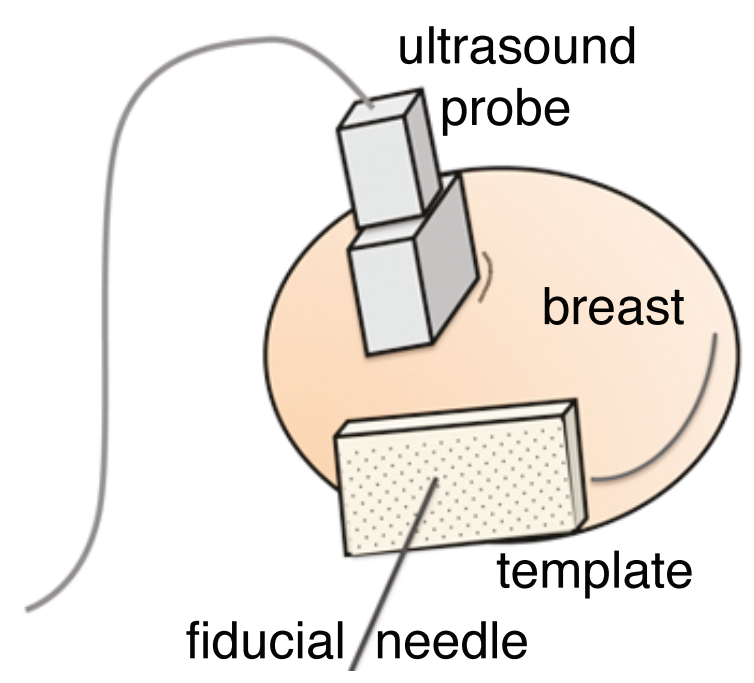

Figure 1.1: Diagram showing permanent breast seed implant procedure

ing mammography. Pignol et al. . $^{[}$developed a permanent seed implant treatment as an alternative to the standard breast radiation in which high-energy $\mathrm{x}$ rays are delivered over a period of 3.5 to 7 weeks $\frac{10}{10}$. With its highly localized dose distributions, brachytherapy is an attractive treatment for early stage cancer.

In this technique ${ }^{6}$, eligible patients with early-stage breast cancer that have received breast-conserving surgery are imaged with breast ultrasound (US) to localize and measure the size of the surgical bed. Computed tomography (CT) imaging is performed so that target volumes and seed placement planning can be performed in a treatment planning system (TPS). A dose of 90 Gy is prescribed to the minimal peripheral dose that covers planning treatment volumes using ${ }^{103} \mathrm{Pd}$ seeds spaced $1 \mathrm{~cm}$ apart in a volume. Seeds are implanted in anesthetized patients using a fiducial needle under ultrasound guidance with the help of a needle spacing template immobilized by a mechanical arm (see figure 1.1). CT images acquired 2 months post-implant are used to identify seed positions and perform quality assurance. 


\subsubsection{Intraoperative brachytherapy for stage I non-small cell lung carcinoma}

In both Canada and the U.S., lung cancer is the leading cause of cancer death 1112 . While the standard treatment for stage I non-small cell lung cancer is lobectomy (i.e., the removal of an entire lobe of the lung) for many patients this treatment poses too great a risk due to poor pulmonary reserve and so sublobar resection (i.e., the removal of only the tumour plus a margin) is used 13 . Unfortunately, limited resections have been associated with a larger incidence of local recurrence ${ }^{14}$. External beam radiotherapy can be used to reduce the higher rate of local recurrence associated with sublobar resection compared to lobectomy, but this can also increase morbidity due to cardiac toxicity, lung fibrosis, and loss of pulmonary function $\frac{15}{}$.

In recent years, ${ }^{125} \mathrm{I}$ brachytherapy has been used in conjunction with sublobar resection to treat stage I non-small cell lung cancer and has been reported to improve disease-free and overall survival rates compared with resection alone. $\frac{8|9| 15 / 20}{20}$ In fact, the use of ${ }^{125} \mathrm{I}$ brachytherapy has been reported to reduce local recurrence of cancer after sublobar resection from $18.6 \%$ to $2 \%$ (Ref. 17) and elsewhere has been described as being a conformal radiation therapy without the concerns of treatment errors due to breathing ${ }^{15}$. While the majority of treatments are performed using ${ }^{125} \mathrm{I}$ seeds, the use of additional radionuclides such as ${ }^{131} \mathrm{Cs}$ (Ref. 21) and ${ }^{169} \mathrm{Yb}$ (Ref. 22) are also under investigation.

In one brachytherapy technique ${ }^{23}$, an implant is created during the surgical procedure by weaving strands of ${ }^{125} \mathrm{I}$ seeds into a vicryl mesh which is then sutured over the resection staple line with the goal of delivering 100 Gy at 5 to $7 \mathrm{~mm}$ along the central axis of the resection margin (see fig. 1.2). Post-treatment CT images are used to identify seed positions and contour suture lines and volumes of interest. 


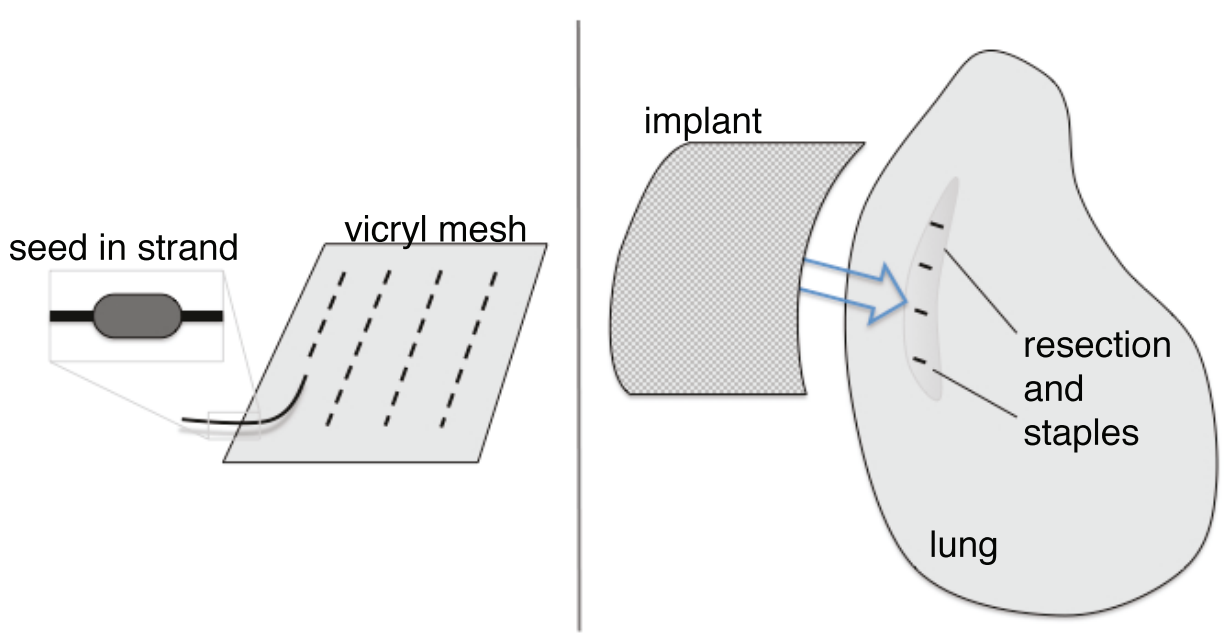

Figure 1.2: Diagram showing lung brachytherapy implant procedure

\subsection{Current clinical brachytherapy dosimetry practice: TG-43}

Dosimetry is the practice of calculating or measuring absorbed-dose (i.e., energy absorbed per unit mass) due to radiation in some medium. In brachytherapy, dosimetry calculation practices are used to determine the distribution of sources in defined volumes to achieve clinically acceptable dose distributions, calculate patient doses, and provide a system for dose prescription 24 .

The current clinical approach for brachytherapy dose calculation is performed using the formalism described by the recommendations of the AAPM Radiation Therapy Committee Task Group No. 43 (TG-43) 25 . The TG-43 formalism uses an analytic expression to calculate the dose in a homogeneous, liquid water environment at any point surrounding a single brachytherapy seed. The expression uses a polar coordinate system along the long axis of sources that are assumed to be cylindrically symmetric. Parameters of the expression are either determined experimentally or using Monte Carlo methods. Dose distributions for implants with multiple seeds are calculated by summing the contribution from each seed individually and, conse- 
quently, this approach does not account for the effect that attenuation and scatter of each seed would have on the dose distributions of other seeds.

Briefly, the TG-43 formalism describes the 2D dose distribution surrounding a source as

$$
\dot{D}(r, \theta)=S_{k} \cdot \Lambda \cdot \frac{G_{L}(r, \theta)}{G_{L}\left(r_{0}, \theta_{0}\right)} \cdot g_{L}(r) \cdot F(r, \theta),
$$

where

$S_{K} \quad$ is the air-kerma strength (units of $\mathrm{U}=\mathrm{cGy} \cdot \mathrm{m}^{2} \mathrm{~h}^{-1}$ ) of the source which is traceable to a primary standards dosimetry laboratory and is determined for specific sources,

$\Lambda \quad$ is the dose rate constant (units of $\mathrm{cGy} \cdot \mathrm{h}^{-1} \mathrm{U}^{-1}=\mathrm{cm}^{-2}$ ) which is the dose rate to water at the reference point per unit air-kerma strength,

$G_{L}(r, \theta)$ is the geometry function which accounts for the dose rate surrounding a seed resulting only from the distribution of the radioactive material within the source,

$g_{L}(r) \quad$ is the radial dose function which represents the fall off of radiation along the transverse axis due to attenuation and scatter in the source and medium,

$F(r, \theta)$ is the anisotropy function which represents the polar variation in dose rate due to attenuation and scatter in the source and medium at a constant radius.

The coordinate system used in the TG-43 formalism can be seen in figure $1.3, r_{0}$ is defined as a distance of $1 \mathrm{~cm}$ from the centre of the seed along the transverse axis and $\theta_{0}$ is defined as an angle of 0 degrees from the transverse axis. Further details of the formalism are described in the 2004 AAPM TG-43U1 report ${ }^{26}$. 


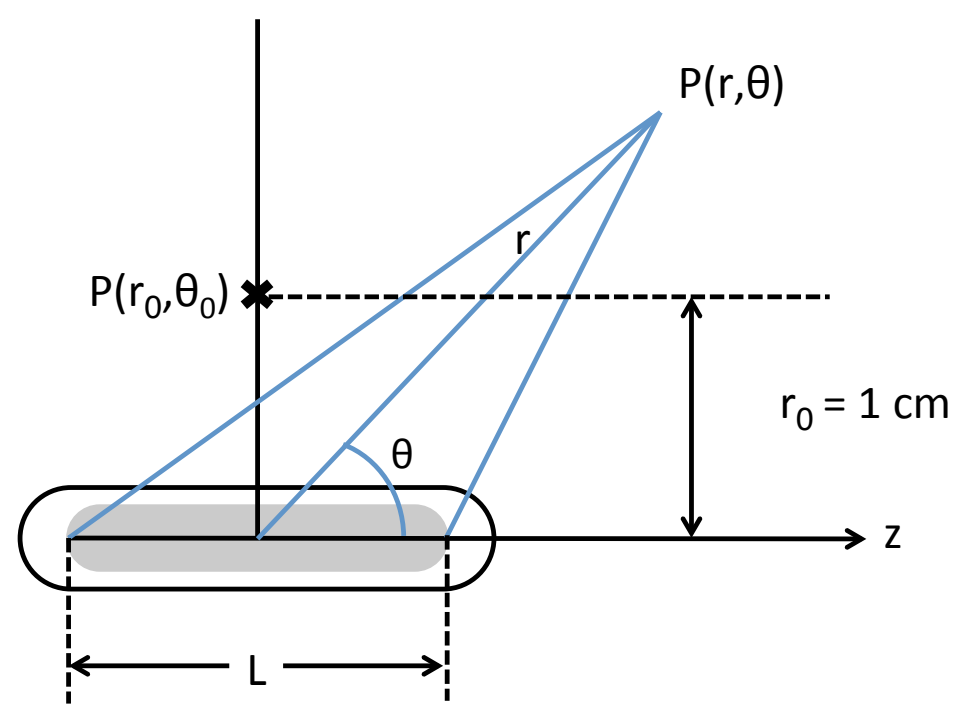

Figure 1.3: Diagram showing the coordinate system used in the TG-43 formalism 26 .

\subsection{Model-based dose calculations for brachytherapy}

While the TG-43 formalism is currently recognized as the world standard for lowenergy brachytherapy dose calculation, it is limited in its ability to produce dose distributions that accurately reconstruct physical dose distributions in patients: the effects of tissue heterogeneities, interseed attenuation, and the finite patient dimensions are all ignored. A review of the literature performed by Rivard et al. concluded that, as a consequence of the limitations of the TG-43 formalism, accepted clinical dose parameters may be over- and under-estimated by as much as $10 \%$ or more 24 . An alternative to water-only calculation geometries is provided by model-based dose calculation algorithms (MBDCA) which model radiation transport and dose deposition in tissues and other non-water media.

Current clinical practices for dose calculation of external beam radiation therapies involve frequent use of MBDCAs and this is now the standard of practice for 
modalities such as intensity modulated radiation therapy (IMRT) 28 . This contrasts the relatively rare use of MBDCAs in the brachytherapy community, where heterogeneity correction algorithms and other MBDCAs have only recently been made available $24 \mid 29$.

Photon interaction processes in the energy range of external beam radiation therapy are dominated by Compton scattering while those for the photon energies of low energy brachytherapy are dominated by the photoelectric effect. Consequently, the differences in the attenuation and scatter of photons and absorbed dose between water and non-water media will be significantly more dramatic for brachytherapy dose calculations. This is illustrated in figure 1.4 which shows the mass attenuation and energy absorption ratios of water to adipose, bone, and muscle as a function of photon energy. The energy range for the common low energy brachytherapy sources is highlighted.

The inability of the water-based TG-43 approach to accurately calculate dose distributions in patient geometries underlines the need for accurate model-based dose calculations for low-energy permanent implant brachytherapy.

\subsection{Monte Carlo simulations}

The Monte Carlo (MC) method of dose calculation, considered to be the current state of the art in computational dosimetry, is based on the sampling of known probability distributions to simulate trajectories and interactions of particle histories to estimate absorbed dose. Consequently, it has the ability to account for interseed effects, calculate absorbed dose to tissue rather than water, simulate dose calculations in heterogeneous media, and to model patient specific geometries.

Recently, Monte Carlo methods have been used to perform explorative and ret- 


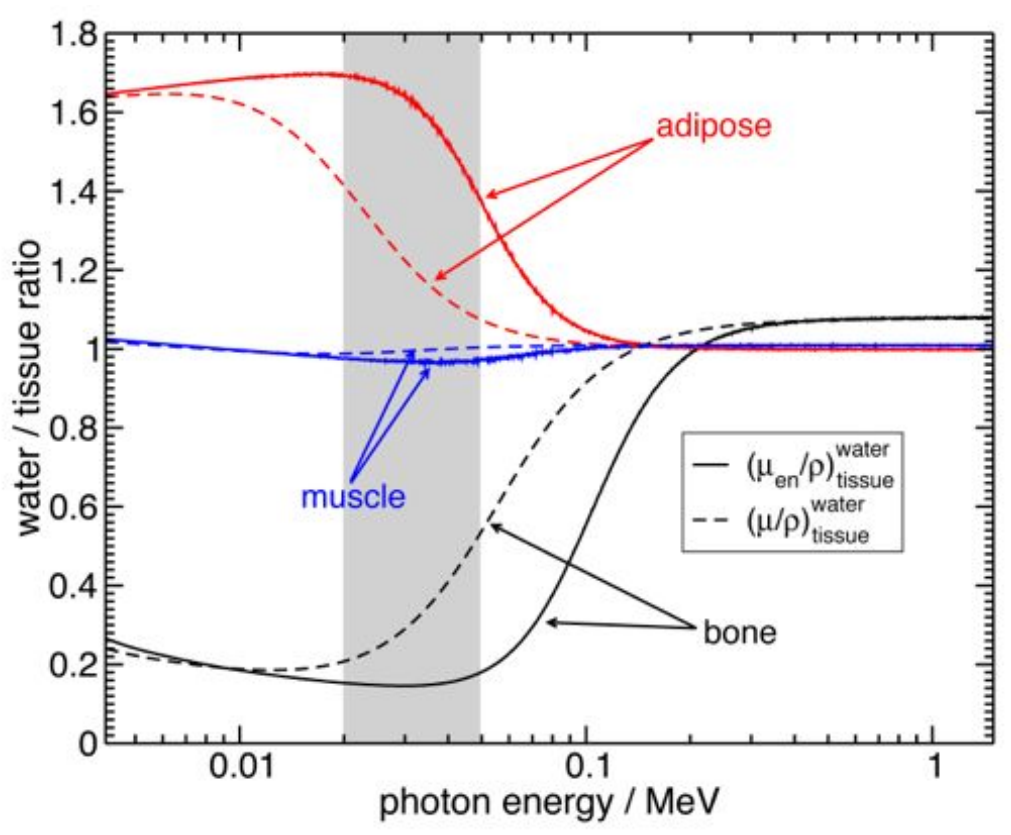

Figure 1.4: Mass attenuation and energy absorption ratios of water to adipose, bone and muscle. The energy range for the common low energy brachytherapy sources is highlighted. Mass attenuation coefficients are taken from the $\mathrm{XCOM}^{30}$ database and mass-energy absorption coefficients are calculated using the EGSnrc user-code $g$.

rospective studies of brachytherapy dosimetry. Interseed attenuation was shown to significantly change dose distributions compared to those calculated by the TG- 43 formalism ${ }^{31} 35$. Numerous studies investigated the effects of non-water tissue compositions and heterogeneities on dose distributions ${ }^{34 \mid 36} 42$. However, despite this significant research, the application of MBDCAs to brachytherapy is still in a state of infancy: a number of issues are unresolved and, in some cases, completely unexplored.

Recent model-based practices in Monte Carlo simulations for brachytherapy have sometimes involved modelling two or more distinct tissue types as a single homogeneous averaged tissue. This has been particularly common in Monte Carlo studies of permanent breast seed implant brachytherapy where averaged tissues have been used to represent different ratios of glandular and adipose tissue ${ }^{40141}$ with CT data 
sometimes being used to assign the mass density to each voxel ${ }^{41}$. The question of the accuracy of using homogeneous averaged tissues in breast calculations (and for low energy brachytherapy in general) and under which conditions their use might be justified in lieu of fully segmented phantoms has not been thoroughly investigated. Additionally, the modelling of calcifications in breast and prostate patient geometries has only seen preliminary treatment in the literature $\sqrt{37 / 43}$.

Patient specific MBDCs generally require that interaction cross sections be assigned on a voxel-by-voxel basis. CT imaging is the standard for fulfilling this need, but unfortunately, provides only voxel electron density which does not relate directly to elemental composition and density. While the assignment of tissues of distinct atomic composition to voxels based on CT number has been investigated in the context of external beam radiation therapy $\underline{44} \underline{47}$, very little focus has been made on the dosimetric results of this practice for the energy ranges of interest in brachytherapy 48 . Additionally, the presence of metallic brachytherapy seeds in CT images creates streaking artifacts and while the correction of these artifacts has been an active area of research $\underline{49}$, 52 , changes in brachytherapy dose distributions as a result of these considerations have not been reported.

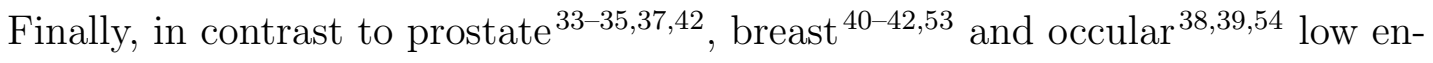
ergy brachytherapy treatments, intraoperative lung brachytherapy has been almost entirely neglected by the MBDCA research community with only one preliminary investigation reported $[55$. Considering the highly heterogeneous treatment sites of lung brachytherapy, the differences between Monte Carlo and TG-43 calculated dose distributions have the potential to be dramatic for these treatments. 


\subsection{Outline of thesis}

The chapters that follow investigate the accurate application of Monte Carlo dose calculations to permanent implant breast and lung brachytherapy. The importance of segmenting tissues in Monte Carlo phantom models is explored. The use of patient CT images for calculating patient specific dose distributions and the challenges that need to be overcome in doing so are presented. Finally, Monte Carlo calculations are used to further the understanding of treatment planning and patient doses for permanent implant lung brachytherapy.

Chapter 2 introduces the EGSnrc Monte Carlo user code BrachyDose used throughout this thesis and then describes the code and methodologies used in this work to produce patient-specific model-based dose calculations.

Chapter 3 begins with a brief review of the literature concerning the application of Monte Carlo dose calculations to permanent breast seed implant brachytherapy and then presents an investigation into the changes of dose due to segmentation of breast tissues. Dose distributions in phantom models with segmented adipose and glandular tissues are compared with those of phantoms consisting of a single averaged tissue. This chapter also investigates the method commonly used in mammography radiation protection calculations to estimate the average dose to glandular tissue by using nonsegmented computational phantoms. Finally, the effect of breast calcifications and the impact that their presence have on the need to segment breast tissues is presented.

Chapter 4 presents the first investigation of patient specific Monte Carlo dose calculations for ${ }^{125} \mathrm{I}$ lung brachytherapy and identifies some of the significant modelling challenges of using CT data to produce computational phantoms. The final effects on dose distributions of metallic artifact correction methods for mitigating streaking 
artifacts in $\mathrm{CT}$ images due to ${ }^{125} \mathrm{I}$ seeds are determined. Additionally, the differences in dose due to the use of different CT to tissue assignment schemes and differences between MC dose distributions and TG-43 calculated doses are investigated.

As a continuation of chapter 4 which identifies the salient modelling challenges, chapter 5 investigates improved techniques for lung brachytherapy patient modelling and dose calculation. A comparison of several metallic artifact reduction techniques and the effect of constraining tissue assignment based on lung contours is described.

Chapter 6 describes an investigation of current lung brachytherapy treatment planning practices which are performed with TG-43 based calculations in a simplified geometry. A method of generating patient-specific virtual implant models is introduced and is used to determine the magnitude of deviations between planned and delivered doses using current treatment planning practices. Calculations are performed using ${ }^{125} \mathrm{I}$ and ${ }^{131} \mathrm{Cs}$ and the effect that source spectra have on doses and dose deviations are discussed.

Using the virtual implant method introduced in chapter 6, Monte Carlo calculated doses to treatment volumes and organs at risk for lung brachytherapy are investigated in chapter $7 .{ }^{125} \mathrm{I},{ }^{103} \mathrm{Pd}$, and ${ }^{131} \mathrm{Cs}$ seeds as well as $50 \mathrm{keV}$ and $100 \mathrm{keV}$ mono-energetic sources are used to identify the relationship between absolute doses, deviations from TG-43 calculated doses and radionuclide spectra.

Finally, concluding remarks are presented in chapter 8. 


\section{Chapter 2}

\section{Model-based dose calculations for brachytherapy}

This chapter presents the Monte Carlo codes and methodologies that are used throughout this thesis to produce patient-specific model-based dose calculations of permanent implant breast and lung brachytherapy.

\subsection{EGSnrc}

EGSnrc (Electron Gamma Shower) ${ }^{[56157}$ is a Fortran/Mortran based Monte Carlo code system, adapted from its predecessor, EGS4 ${ }^{58}$, that simulates coupled photon and electron transport in the $\mathrm{keV}$ to $\mathrm{GeV}$ energy range.

Photon interactions in the EGSnrc code include Rayleigh scattering with atoms (or molecules), photoelectric absorption, Compton scattering, and pair and triplet production. These interactions are described in detail in Attix ${ }^{59}$, Podgorsak ${ }^{60}$, and Johns and Cunningham $\frac{61}{2}$. EGSnrc also simulates the production of characteristic $\mathrm{x}$ rays from photon and electron interactions. The most accurate cross sections are attained by using the NIST XCOM compilation of cross sections ${ }^{62}$. Electrons and positrons are simulated using the condensed history approach $\frac{6364}{6}$, which condenses the cumulative effect of many energy loss interactions by randomly sampling from 
multiple scattering distributions and improves simulation efficiency compared to single scattering calculations. Spin effect, density effects, and electron impact ionization are modelled in addition to basic charged particle interactions $\underline{65}$. By using restricted stopping powers and by including bremsstrahlung photons above a cutoff energy, AP, and secondary electrons above a cutoff energy, AE, simulations account for radiative losses and collision energy loss interactions.

\subsection{BrachyDose}

User codes are written to interface with the EGSnrc code system so that calculations may be performed. BrachyDose ${ }^{\sqrt{66 \mid 67}}$ is a recent EGSnrc user code for brachytherapy calculations and facilitates significant improvements over previous EGS-based brachytherapy calculations $\frac{68}{75}$ that relied on the EGS4 system and did not have a general purpose geometry package. BrachyDose allows for accurate modelling of rectilinear, spherical, cylindrical, and conical geometries by making use of Yegin's multi-geometry package ${ }^{76}$.

To improve calculation times, BrachyDose makes approximations that take advantage of the the low-energy regime of brachytherapy. In addition to calculating deposited energy through the interactions of simulated secondary electrons as is done in most EGSnrc user codes, BrachyDose estimates absorbed-dose as collision kerma (kinetic energy release per mass) in a medium. For the energy range of interest, collision kerma is a good approximation of absorbed dose since the range of secondary electrons (e.g., $\sim 0.013 \mathrm{~mm}$ for $25 \mathrm{keV}$ electrons in water) is much less than typical phantom voxel sizes $\left(\gtrsim 0.5 \mathrm{~mm}^{3}\right)$ and so the energy of these electrons can be considered to be deposited locally and are generally not tracked. BrachyDose uses a 
tracklength estimator to score collision kerma per history;

$$
D^{j}=K_{c o l}^{j}=\sum_{i} E_{i} t_{i}\left(\frac{\mu_{e n}}{\rho}\right)_{i} / V_{j},
$$

where, for photon $i$ and voxel $j, D^{j}$ and $K_{c o l}^{j}$ are the dose and collision kerma of the voxel, $E_{i}$ is the energy of the photon, $t_{i}$ is the track length of the photon in the voxel, $\left(\frac{\mu_{e n}}{\rho}\right)_{i}$ is the mass energy absorption coefficient of energy $E_{i}$, and $V_{j}$ is the volume of the voxel. While scoring collision kerma using a track length estimator is significantly more computationally efficient, BrachyDose is also capable of electron tracking and interaction scoring if desired.

The majority of commercially available brachytherapy seeds, including the seeds used in this thesis, have previously been fully modelled with the multi-geometry package $^{\sqrt{76}}$ using measured dimensions and manufacturer specifications ${ }^{66}$. Extensive benchmarking of seed models and BrachyDose has been performed via calculations and comparison with reported values of the TG-43 dosimetry parameters for these seeds 66 66/77+79.

\subsection{Calculating absolute dose}

To calculate patient specific doses and to compare Monte Carlo calculated and TG-43 derived doses, it is necessary to convert the values of dose per history calculated by BrachyDose into absolute dose. For permanent implant treatments, this conversion is given by

$$
D_{\mathrm{abs}}=D_{\mathrm{BD}} \cdot \frac{1}{s_{K}} \cdot S_{K} \cdot \tau
$$

where $D_{\text {abs }}$ is absolute dose, $D_{\mathrm{BD}}$ is dose per history (given by BrachyDose), $s_{K}$ is 
the air-kerma strength per history, $S_{K}$ is the initial air-kerma strength of the sources in the treatment, and $\tau$ is the mean lifetime of the source radionuclide. The method for calculating the air-kerma strength per history for a particular seed is described by Taylor et al. .66

Doses to various media in heterogeneous geometries differ from doses in homogeneous water geometries as a result of two effects: the differences in photon energy fluence (particle transport) and differences in energy absorbed per unit energy fluence by non-water media (dose scoring). Consequently, it can be instructive to isolate the effect of media heterogeneity on photon energy fluence from the effect of media heterogeneity on absorbed dose by calculating dose to fictitious water (or other media) voxels in a heterogeneous phantom. BrachyDose estimates dose by calculating collision kerma in a medium which, for a monoenergetic beam, is given by

$$
\left(K_{c}\right)_{\mathrm{m}}=\Psi\left(\frac{\mu_{e n}}{\rho}\right)_{\mathrm{m}}
$$

where $\left(K_{c}\right)_{\mathrm{m}}$ is collision kerma (in medium $\left.\mathrm{m}\right), \Psi$ is the photon energy fluence, and $\left(\frac{\mu_{e n}}{\rho}\right)_{\mathrm{m}}$ is the mass energy absorption coefficient for the medium. Thus, one calculates dose to a fictitious voxel medium by substituting the mass energy absorption coefficient for the fictitious medium of the voxel in place of that of the actual voxel medium in equation 2.1. The concept of dose to a fictitious voxel medium is illustrated in figure 2.1 .

The uncertainty on doses calculated by BrachyDose will be affected by the uncertainty of the $\mathrm{XCOM}^{[30}$ cross section uncertainties. Hubbell estimated that the uncertainty on mass attenuation coefficients is $\pm 5 \%$ for photons below $5 \mathrm{keV}$ and $\pm 2 \%$ for photons up to $10 \mathrm{MeV}^{80}$. However, given the assumption that errors in cross sections are correlated, the effect of cross section uncertainty on the results and 


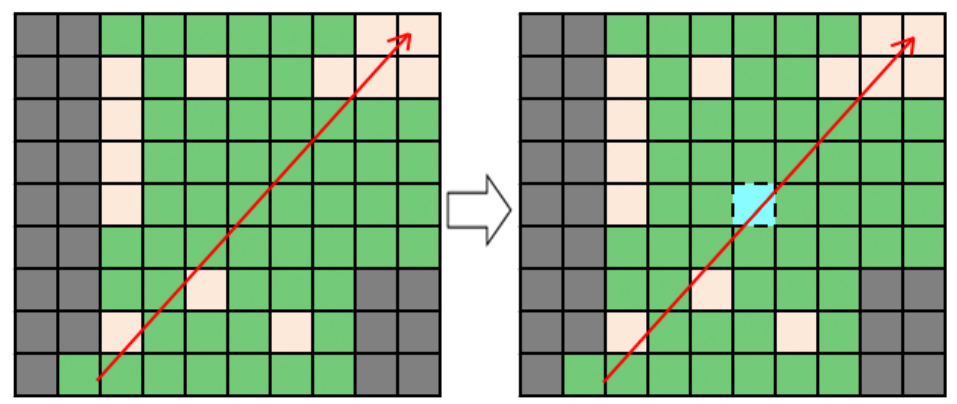

Figure 2.1: Diagram depicting calculation of dose to a fictitious voxel medium. The left panel shows a phantom of various media with a photon track calculated in those media. In the right panel, when the contribution of that photon track to the dose in the voxel (surrounded by dashed lines) is calculated, the mass energy absorption coefficient for another medium is used and so dose to a voxel of that medium is scored.

conclusions presented in this work will be minimal since the main interest is in dose differences rather than absolute dosimetry.

\subsection{Using patient data for BrachyDose calculations}

Patient specific model-based dose calculations are performed by creating a model of the patient geometry. This is most commonly achieved by modelling interaction cross sections on a voxel-by-voxel basis; each voxel is assigned a mass density and a tissue with a particular elemental composition.

CT images are routinely used to create patient specific computational phantoms since they display anatomy and inhomogeneity and pixel values include quantitative information about the radiological properties of tissues. CT image pixel values or CT numbers are expressed in Hounsfield units (HU) and are calculated using

$$
H=1000\left(\frac{\mu}{\mu_{\text {water }}}-1\right)
$$


where $H$ is the CT number, $\mu$ is the linear attenuation coefficient of the voxel, and $\mu_{\text {water }}$ is the linear attenuation coefficient of water. Thus, CT number is defined such that water has a value of 0 and air has a value of -999.2 at $20 \mathrm{keV}$.

While accurate Monte Carlo simulations of higher energy external beam radiation therapy can be achieved by assigning each phantom voxel an electron density determined from the CT number $\underline{8182}$, the considerable influence of the photoelectric effect at the photon energies of low-energy brachytherapy makes it necessary that the mass density and elemental composition of voxels be defined for MBDCs of brachytherapy. The average mass density of a voxel can be determined by using a scanner specific CT to mass density calibration that is determined experimentally $\sqrt[83]{ }$. However, the assignment of voxel elemental compositions based on CT numbers is more challenging. This has commonly been achieved by assigning a small number of discrete tissues with defined elemental compositions based on the mass density (or, equivalently, CT number) of each voxel ${ }^{45|48| 84}$ but a more accurate stoichiometric calibration has also been described $\underline{44}$.

\subsubsection{Elemental composition of voxels}

Table 2.1 lists the tissue compositions taken from Woodard and White ${ }^{\sqrt{5}}$ and White

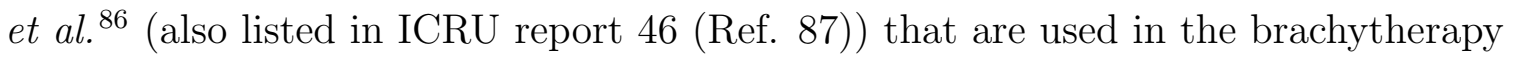
phantoms in this thesis.

\subsubsection{Tools for files in DICOM format}

Clinical patient data files are most often stored in a format that follows the international standard, DICOM (Digital Imaging and Communications in Medicine) ${ }^{88}$ which 
Table 2.1: Elemental compositions and mass densities of tissues (from Woodard and White 1986(Ref. 85), White et al. ${ }^{86}$ ). Composition of air (for $40 \%$ relative humidity) and breast calcification are taken from TG-43(Ref. 26) and ICRU report 46(Ref. 87), respectively.

\begin{tabular}{|c|c|c|c|c|c|c|}
\hline \multirow[b]{2}{*}{ Material } & \multicolumn{5}{|c|}{ Composition (Mass \%) } & \multirow{2}{*}{$\begin{array}{c}\rho \\
\left(\mathrm{g} / \mathrm{cm}^{3}\right)\end{array}$} \\
\hline & $\mathrm{H}$ & $\mathrm{C}$ & $\mathrm{N}$ & $\mathrm{O}$ & Elements with $\mathrm{Z}>8$ & \\
\hline Adipose 1 & 11.2 & 51.7 & 1.3 & 35.5 & $\mathrm{Na}(0.1), \mathrm{S}(0.1), \mathrm{Cl}(0.1)$ & 0.97 \\
\hline Adipose 2 & 11.4 & 59.8 & 0.7 & 27.8 & $\mathrm{Na}(0.1), \mathrm{S}(0.1), \mathrm{Cl}(0.1)$ & 0.95 \\
\hline Adipose 3 & 11.6 & 68.1 & 0.2 & 19.8 & $\mathrm{Na}(0.1), \mathrm{S}(0.1), \mathrm{Cl}(0.1)$ & 0.93 \\
\hline Air (TG-43) & 0.07 & 0.01 & 75.03 & 23.61 & $\operatorname{Ar}(1.27)$ & 0.0012 \\
\hline Aorta & 9.9 & 14.7 & 4.2 & 69.8 & $\mathrm{Na}(0.2), \mathrm{P}(0.4), \mathrm{S}(0.3), \mathrm{K}(0.1), \mathrm{Ca}(0.4)$ & 1.05 \\
\hline Blood - whole & 10.2 & 11.0 & 3.3 & 74.5 & $\begin{array}{c}\mathrm{Na}(0.1), \mathrm{P}(0.1), \mathrm{S}(0.2), \mathrm{Cl}(0.3), \mathrm{K}(0.2), \\
\mathrm{Fe}(0.1)\end{array}$ & 1.06 \\
\hline Breast calcification & 0.3 & 1.6 & 0.5 & 40.7 & $\mathrm{P}(18.7), \mathrm{Ca}(38.2)$ & 3.06 \\
\hline Cartilage & 9.6 & 9.9 & 2.2 & 74.4 & $\mathrm{Na}(0.5), \mathrm{P}(2.2), \mathrm{S}(0.9), \mathrm{Cl}(0.3)$ & 1.1 \\
\hline Cortical bone & 3.4 & 15.5 & 4.2 & 43.5 & $\begin{array}{c}\mathrm{Na}(0.1), \operatorname{Mg}(0.2), \mathrm{P}(10.3), \mathrm{S}(0.3) \\
\mathrm{Ca}(22.5)\end{array}$ & 1.92 \\
\hline Heart 1 & 10.3 & 17.5 & 3.1 & 68.1 & $\mathrm{Na}(0.1), \mathrm{P}(0.2), \mathrm{S}(0.2), \mathrm{Cl}(0.2), \mathrm{K}(0.3)$ & 1.05 \\
\hline Heart 3 & 10.4 & 10.3 & 2.7 & 75.6 & $\mathrm{Na}(0.1), \mathrm{P}(0.2), \mathrm{S}(0.2), \mathrm{Cl}(0.2), \mathrm{K}(0.3)$ & 1.05 \\
\hline $\begin{array}{c}\text { Heart } \\
\text {-blood-filled }\end{array}$ & 10.3 & 12.1 & 3.2 & 73.4 & $\begin{array}{c}\mathrm{Na}(0.1), \mathrm{P}(0.1), \mathrm{S}(0.2), \mathrm{Cl}(0.3), \mathrm{K}(0.2) \\
\mathrm{Fe}(0.1)\end{array}$ & 1.06 \\
\hline $\begin{array}{l}\text { Lung } \\
\text {-blood-filled }\end{array}$ & 10.3 & 10.5 & 3.1 & 74.9 & $\begin{array}{c}\mathrm{Na}(0.2), \mathrm{P}(0.2), \mathrm{S}(0.3), \mathrm{Cl}(0.3) \\
\mathrm{K}(0.2)\end{array}$ & 0.26 \\
\hline Mammary gland 2 & 10.6 & 33.2 & 3.0 & 52.7 & $\mathrm{Na}(0.1), \mathrm{P}(0.1), \mathrm{S}(0.2), \mathrm{Cl}(0.1)$ & 1.02 \\
\hline Muscle 1 & 10.1 & 17.1 & 3.6 & 68.1 & $\mathrm{Na}(0.1), \mathrm{P}(0.2), \mathrm{S}(0.3), \mathrm{Cl}(0.1), \mathrm{K}(0.4)$ & 1.05 \\
\hline Muscle 2 & 10.2 & 14.3 & 3.4 & 71.0 & $\mathrm{Na}(0.1), \mathrm{P}(0.2), \mathrm{S}(0.3), \mathrm{Cl}(0.1), \mathrm{K}(0.4)$ & 1.05 \\
\hline Muscle 3 & 10.2 & 11.2 & 3.0 & 74.5 & $\mathrm{Na}(0.1), \mathrm{P}(0.2), \mathrm{S}(0.3), \mathrm{Cl}(0.1), \mathrm{K}(0.4)$ & 1.05 \\
\hline Red marrow & 10.5 & 41.4 & 3.4 & 43.9 & $\mathrm{P}(0.1), \mathrm{S}(0.2), \mathrm{Cl}(0.2), \mathrm{K}(0.2), \mathrm{Fe}(0.1)$ & 1.03 \\
\hline Ribs (2nd, 6th) & 6.4 & 26.3 & 3.9 & 43.6 & $\begin{array}{c}\mathrm{Na}(0.1), \operatorname{Mg}(0.1), \mathrm{P}(6.0), \mathrm{S}(0.3), \mathrm{Cl}(0.1), \\
\mathrm{K}(0.1), \mathrm{Ca}(13.1)\end{array}$ & 1.41 \\
\hline Ribs (10th) & 5.6 & 23.5 & 4.0 & 43.4 & $\begin{array}{c}\mathrm{Na}(0.1), \operatorname{Mg}(0.1), \mathrm{P}(7.2), \mathrm{S}(0.3), \mathrm{Cl}(0.1), \\
\mathrm{K}(0.1), \mathrm{Ca}(15.6)\end{array}$ & 1.41 \\
\hline Skin 1 & 10.0 & 25.0 & 4.6 & 59.4 & $\mathrm{Na}(0.2), \mathrm{P}(0.1), \mathrm{S}(0.3), \mathrm{Cl}(0.3), \mathrm{K}(0.1)$ & 1.09 \\
\hline Skin 2 & 10.0 & 20.4 & 4.2 & 64.5 & $\mathrm{Na}(0.2), \mathrm{P}(0.1), \mathrm{S}(0.2), \mathrm{Cl}(0.3), \mathrm{K}(0.1)$ & 1.09 \\
\hline Skin 3 & 10.1 & 15.8 & 3.7 & 69.5 & $\mathrm{Na}(0.2), \mathrm{P}(0.1), \mathrm{S}(0.2), \mathrm{Cl}(0.3), \mathrm{K}(0.1)$ & 1.09 \\
\hline Water & 11.22 & 0.0 & 0.0 & 88.78 & & 0.998 \\
\hline Yellow Marrow & 11.5 & 64.4 & 0.7 & 23.1 & $\mathrm{Na}(0.1), \mathrm{S}(0.1), \mathrm{Cl}(0.1)$ & 0.98 \\
\hline
\end{tabular}


has seen widespread adoption in hospitals and vendor software. To facilitate the use of clinical patient files for the calculations in this thesis, $\mathrm{C}++$ tools were developed to interact with four DICOM file types:

CT image files: A tool set named "ct_tools" was developed that reads in DICOM CT images and converts them to an in-house, text-based data structure. The in-house data structure is used to allow the application of image treatment algorithms (see chapter 4) and to change phantom sizes through cropping or resampling. With a user supplied CT mass density calibration and tissue assignment scheme, the CT data is converted to the EGSnrc .egsphant file type which defines phantom voxel boundaries and 3D arrays of voxel tissue assignments and mass densities.

RT dose files: Part of the extension of version 3.0 of the DICOM standard that deals with radiotherapy file types, patient RT dose files contain dose distributions calculated by treatment planning systems. These files are used to read DICOM meta-data that are required when converting BrachyDose output into DICOM format.

RT plan files: These files contain the seed positions, radionuclides, and air-kerma strengths of brachytherapy treatments that are being simulated. This information is used to help generate input files for BrachyDose.

RT structure files: Contours defining patient organs and treatment volumes are defined in this file type. These contours are used to calculate dose volume histograms and clinical dose metrics as well as guiding the assignment of tissues when converting from CT to .egsphant (see chapter 5). 


\subsection{Simulation parameters}

The majority of the simulations in this thesis are performed with the same parameters. Seeds are fully modelled ${ }^{[77}$ and, in most cases, are superimposed on a voxelized geometry specified by EGSnrc phantom (.egsphant) files. Seed models, unless otherwise stated, were previously benchmarked via calculations of the TG-43 dosimetry parameters $\frac{66}{}$. For all calculations, the photon energy cutoff is set to $1 \mathrm{keV}$ and Rayleigh scattering, bound Compton scattering, photoelectric absorption, and fluorescent emission of characteristic $\mathrm{K}$ and L-shell $\mathrm{x}$ rays are modelled. Photon cross sections are taken from the $\mathrm{XCOM}^{30}$ database and mass-energy absorption coefficients are calculated using the EGSnrc user-code $g$. Note that electron transport is not modelled and dose in voxels is approximated by collision kerma. 


\section{Chapter 3}

\section{Breast tissue segmentation}

Breast tissue generally consists of fibroglandular and adipose tissues, possibly with some calcifications. The proportion of each of these tissues in a typical breast has been studied by Yaffe et al. $\frac{89}{6 h}$ wh found that the mean percentage of fibroglandular tissue was $19.3 \%$ by volume. The dose to fibroglandular tissue is the quantity of interest in mammography radiation protection ${ }^{89}$ and may also be relevant for brachytherapy treatments as the linear attenuation coefficients of gland and tumour are similar $\underline{93 \mid 94}$. With the advent of ${ }^{103} \mathrm{Pd}$ treatments, the use of $50 \mathrm{kV}$ electronic brachytherapy sources for partial breast irradiation, and the use of model-based dose calculation algorithms, there is increasing interest in the role of breast tissue composition in brachytherapy $\underline{4014195}$.

At the time of the research presented in this chapter, current model-based practices in Monte Carlo simulations for brachytherapy typically used homogeneous averaged tissues to represent different ratios of glandular and adipose tissue 40411 . Sometimes, CT data were used to assign mass density to each voxel $\stackrel{41}{4}$. Photon transport and energy deposition occured in these averaged tissues. While more recent work began to investigate breast tissue segmentation ${ }^{96}$ and the importance of tissue segmentation for kilovoltage beams ${ }^{97}$, the differences between the dose to the separate glandular and adipose tissues had been largely ignored in treatment planning studies. 
In mammography radiation protection, it is common to estimate the average

dose to the glandular tissue $\mathrm{e}^{82}$ by transporting photons through an averaged tissue and then calculating the portion of energy deposited in the fibroglandular tissue using ratios of mass energy absorption coefficients. In effect, photon transport is modelled in the averaged tissue and energy deposition to gland is calculated.

Prior to the work presented in this chapter, the question of the accuracy of using homogeneous averaged tissues in breast calculations and under which conditions their use might be justified in lieu of fully segmented phantoms had not yet been thoroughly investigated. This chapter presents an investigation of the effects of more realistic segmentation of breast tissues in model-based Monte Carlo breast dosimetry. Possible inaccuracies may occur during the transport of particles through the breast creating differences in the photon energy fluence, and during the deposition of dose because of choices of dose scoring media and incorrect photon energy fluence. In this chapter, the current model-based practices of brachytherapy and mammography radiation protection are investigated and compared to fully segmented calculations. The modelling of calcifications in the breast is also investigated.

\subsection{Methods}

In all calculations, sixty-four fully modelled ${ }^{77}$ TheraSeed (Theragenics Corporation, Buford Georgia USA) $200{ }^{103} \mathrm{Pd}$ brachytherapy seeds (mean emerging photon energy from seed of $20.71 \mathrm{keV}$ (Ref. 98)) are placed in a cube formation centred around the centre of the phantom, $(0,0,0) \mathrm{cm}$, with central $\mathrm{x}, \mathrm{y}$, and $\mathrm{z}$ coordinates of $\pm 1.55 \mathrm{~cm}$ or $\pm 0.55 \mathrm{~cm}$ and axes parallel to the z-axis. The $0.05 \mathrm{~cm}$ offsets were chosen so that the centres of the seeds would not lie on voxel boundaries for $(1 \mathrm{~mm})^{3}$ voxel sizes. A $64 \mathrm{~cm}^{3}$ planning treatment volume (PTV) region is defined as a cube ranging from 
$(2,2,2) \mathrm{cm}$ to $(-2,-2,-2) \mathrm{cm}$. As these dimensions represent a larger PTV and lower

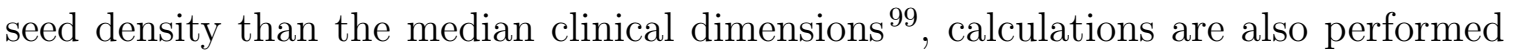
with seed central $\mathrm{x}, \mathrm{y}$, and z coordinates of $\pm 0.92 \mathrm{~cm}$ or $\pm 0.46 \mathrm{~cm}$ with a PTV ranging from $(1.23,1.23,1.23) \mathrm{cm}$ to $(-1.23,-1.23,-1.23) \mathrm{cm}$ to approximate a clinically small, dense treatment.

Simulations of $10^{9}$ histories achieve statistical uncertainties of less than $0.2 \%$ on the dose in voxels in the PTV. These high precision calculations, which each take roughly 4.5 hours of CPU time on a single $3.0 \mathrm{GHz}$ Woodcrest core, are not necessarily needed for the calculation of DVHs as simulations of $10^{7}$ histories produce nearly indistinguishable curves.

Densities and elemental compositions of breast tissues and breast calcifications are taken from Woodard and White (Ref. 85) and ICRU Report 46 (Ref. 87) respectively (table 2.1, page 19]. The averaged breast tissues in this work are specified as percent mixtures by mass. For most calculations, proportions of $25 \%$ fibroglandular tissue and $75 \%$ adipose tissue by mass $(23.7 \%$ and $76.3 \%$ by volume) are used to

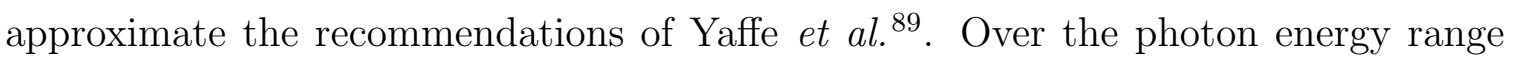
of 10 to $30 \mathrm{keV}$, the mass energy absorption coefficient ratios of fibroglandular tissue, adipose tissue, and a $25 \%$ gland $75 \%$ adipose mixture (by mass) to water are at $0.80 \pm 0.01$ (gland/water), $0.60 \pm 0.01$ (adipose/water), and $0.65 \pm 0.01$ ((25/75 mixture)/water).

Physical dose distributions and dose volume histograms are both calculated. To investigate doses to adipose and fibroglandular tissues separately, an in-house code was developed to allow the calculation of dose volume histograms (DVHs) wherein the volume considered consists only of those voxels within the PTV containing one medium (e.g., DVHs for voxels containing gland only). 


\subsubsection{Dose to gland and adipose versus dose to an averaged tissue}

For this portion of the study a simple geometry configuration defined as a $12 \times 12 \times 12 \mathrm{~cm}^{3}$ phantom with $(1 \mathrm{~mm})^{3}$ voxels is used to approximate a breast brachytherapy treatment; dose distributions are unchanged within statistics with $(2 \mathrm{~mm})^{3}$ voxels. The PTV is at the centre of the phantom. The whole phantom is filled with an averaged tissue of given proportions of gland and adipose or each voxel is randomly assigned a single tissue so as to create a phantom with the same proportion of tissues by mass. For example, one phantom has voxels containing a single averaged tissue ( $25 \%$ gland, $75 \%$ adipose by mass) while the other has each voxel randomly assigned gland or adipose so as to maintain this proportion by mass over the entire phantom. These phantoms are called "25/75-averaged-tissue phantom" and "25/75randomly-segmented phantom" respectively and the averaged tissue is denoted by "25/75-averaged-tissue". In general, the naming scheme used in this work is [proportions of gland/adipose(/calcification) by mass]-[segmentation model]. For the averaged tissue phantoms, dose is scored either in the averaged tissue (to investigate the method often used in brachytherapy calculations) or in fibroglandular tissue (to in-

vestigate the method used for mammography radiation protection studies ${ }^{89}$ albeit with a much different source geometry) (see page 16 for a description of scoring dose to fictitious voxel media). For randomly segmented phantoms, dose is scored in the tissue of each particular voxel.

These phantoms are used to investigate the hypothesis that the photon energy fluence remains relatively unchanged between an averaged tissue phantom and a randomly segmented phantom. Assuming this is the case, these phantoms can provide information concerning dose differences that arise from modelling gland and adipose 


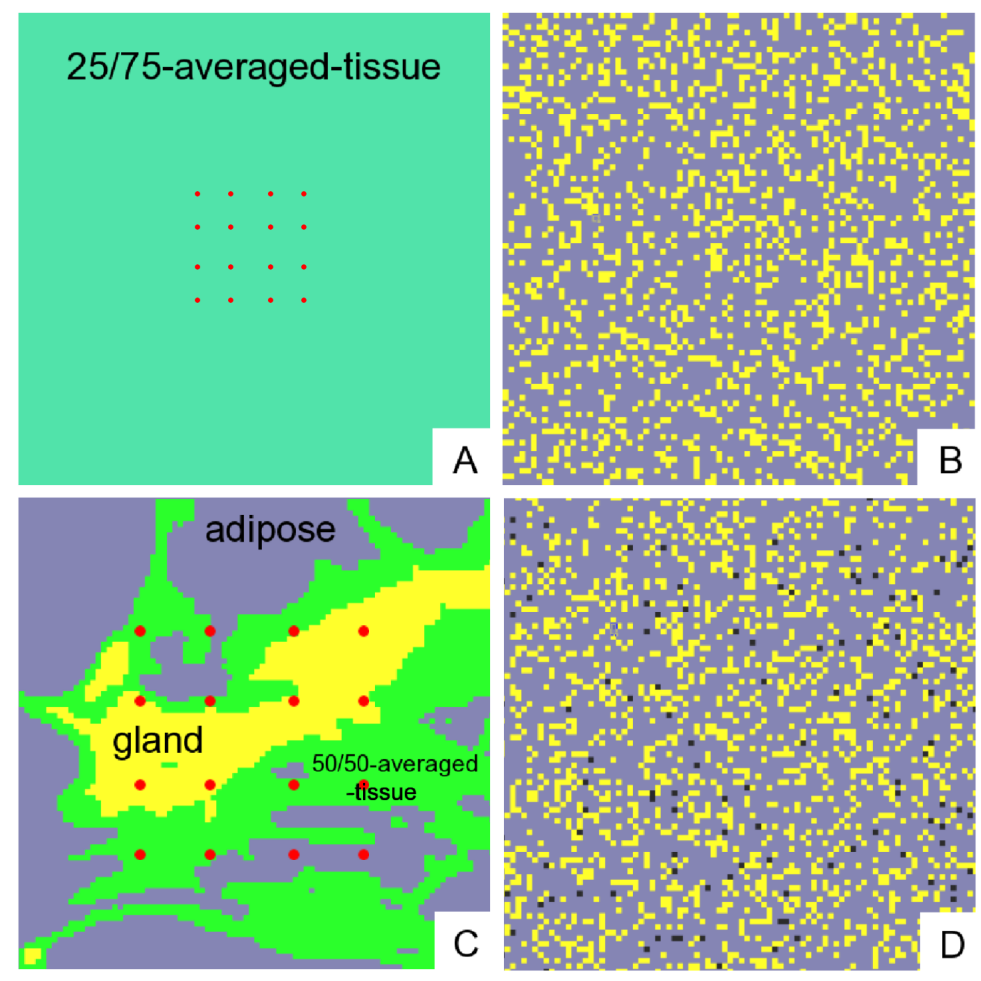

Figure 3.1: Diagrams showing representational $\mathrm{x}-\mathrm{y}$ slices of inner portions of various phantoms. (A) a slice in the $25 / 75$-averaged-tissue phantom. (B) a slice of the 25/75-randomly-segmented phantom, (C) a representational slice from only the detailed inner section of the 26.4/73.6-realistically-segmented phantom and (D) a slice of the 22.5/72.5/5-randomly-segmented phantom. In (B) and (D), yellow (white) represents gland voxels, blue (grey) represents adipose voxels, and black represents calcification. Seed positions are shown in red (dark grey) in (A) and $(\mathrm{C})$ but omitted in (B) and (D) for clarity. The seed positions in (B) and (D) would be the same as in $(A)$.

tissues separately or using an averaged tissue phantom independent of the effects of differing photon energy fluence. Diagrams A and B of figure 3.1 show representational slices of the 25/75-averaged-tissue and 25/75-randomly-segmented phantoms respectively. 


\subsubsection{Effect of realistic segmentation on photon energy fluence}

To approximate a realistically segmented breast, a phantom was created using a numerical breast phantom from the work of Zastrow et al. ${ }^{100}$ Breast phantom 070604PA1 was chosen because its proportions are nearly $25 \%$ gland and $75 \%$ adipose by mass. This phantom contains three classes of fibroglandular and adipose tissues that differ in their dielectric properties. Voxels in the Zastrow phantom containing any class of fibroglandular (adipose) tissue are set to the fibroglandular (adipose) tissue (composition found in table 2.1, page 19) in the phantoms for the present work. The numerical phantom also contains a so-called transitional tissue (having dielectric properties transitioning between gland and adipose) which is approximated in the phantoms for the present work as being $50 \%$ adipose and $50 \%$ fibroglandular tissues by mass. The centre of the phantom for the present work is set to $(0,0,0) \mathrm{cm}$ and a $5 \times 5 \times 5 \mathrm{~cm}^{3}$ centred cube of $0.5 \times 0.5 \times 0.5 \mathrm{~mm}^{3}$ voxels is taken from the numerical phantom of voxels of the same size. The $5 \times 5 \times 5 \mathrm{~cm}^{3}$ detailed cube is surrounded by single, large voxels of adipose tissue that extend to the outer dimensions of the numerical phantom $(\mathrm{x}= \pm 7.5 \mathrm{~cm}, \mathrm{y}= \pm 9.55 \mathrm{~cm}, \mathrm{z}= \pm 6.75 \mathrm{~cm})$. In the detailed cube, $9 \%$ of voxels are gland, $31 \%$ are 50/50 gland/adipose, and $60 \%$ are adipose (see a typical slice in figure 3.1 C). A second phantom is also created that is identical except that the detailed $5 \times 5 \times 5 \mathrm{~cm}^{3}$ cube is filled with voxels of an averaged tissue with the same proportions by mass as the segmented detailed cube (26.4\% fibroglandular and $73.6 \%$ adipose tissue). These phantoms are called "26.4/73.6-realistically-segmented phantom" and "26.4/73.6-averaged-tissue phantom" respectively and the averaged tissue is denoted by "26.4/73.6-averaged-tissue". The position of the seeds and PTV remain the same as those of the randomly segmented phantom as they lie approximately in 
the centre of the distribution of fibroglandular tissue.

To approximate the use of CT data to assign voxel densities, an additional modified averaged tissue phantom is created such that each voxel contains the averaged tissue material but with voxel densities identical to that of the realistically segmented phantom.

A second realistically segmented phantom is also created using another computational phantom from Zastrow et al. to confirm that the general trend of the results found are not dependent on the particular glandular density of the 26.5/73.6realistically-segmented phantom. This denser phantom is composed of approximately $55 \%$ fibroglandular and $45 \%$ adipose tissues.

\subsubsection{The effect of calcifications on photon energy fluence}

A randomly segmented phantom is created composed of $22.5 \%$ fibroglandular tissue, $72.5 \%$ adipose tissue and $5 \%$ calcification by mass $(22.1 \%, 76.3 \%$ and $1.6 \%$ by volume respectively) with calcified $(1 \mathrm{~mm})^{3}$ voxels distributed randomly throughout the entire phantom. The fraction of calcification was chosen based on results found in the literature ${ }^{101 / 102}$. While the dimensions of $1 \times 1 \times 1 \mathrm{~mm}^{3}$ used may be too small to represent the size of an average calcification, it serves well as a limiting case scenario; if small, randomly distributed calcifications significantly effect the ability to approximate the photon energy fluence with an averaged tissue, then larger ones will have an effect. This phantom is compared to an averaged tissue phantom of the same proportions, a second averaged tissue phantom where the density of each voxel matches that of the same voxel in the randomly segmented phantom, and a third averaged tissue phantom composed of $25 \%$ gland, $75 \%$ adipose with the same voxel densities as the randomly segmented phantom (that includes calcifications). The randomly segmented and av- 
eraged tissue phantoms are denoted by "22.5/72.5/5-randomly-segmented phantom" and "22.5/72.5/5-averaged-tissue phantom" respectively and the averaged tissue is denoted by “22.5/72.5/5-averaged-tissue”. Diagram D of figure 3.1 shows a representational slice of the 22.5/72.5/5-randomly-segmented phantom.

\subsection{Results and Discussion}

\subsubsection{Dose to gland and adipose versus dose to an averaged tissue}

Figure 3.2 compares the DVHs for the dose to the gland voxels within the PTV of the 25/75-randomly-segmented phantom to the dose to the voxels corresponding to the same spatial coordinates of the 25/75-averaged-tissue phantom with dose scored in gland. The close agreement between the two curves implies that the media in the 25/75-randomly-segmented phantom are sufficiently uniformly distributed such that, to first order, the photon energy fluence remains the same between the two phantoms. This is confirmed by the fact that the explicitly calculated photon energy fluences at the centre of each phantom agree within $2 \%$. The agreement also implies that, in the very unlikely case that a realistic breast is also sufficiently uniform in its distribution of gland and adipose tissues (i.e., the photon energy fluence of a realistic breast remains unchanged from that of an averaged tissue phantom), the method used in mammography of photon transport in an averaged tissue and dose scoring in a fibroglandular tissue would provide accurate dose volume metrics and mean doses to glandular tissue.

Figure 3.3 compares the DVHs for the dose to the entire PTV for the 25/75randomly-segmented phantom (dose to gland and adipose voxels) and 25/75-averaged- 


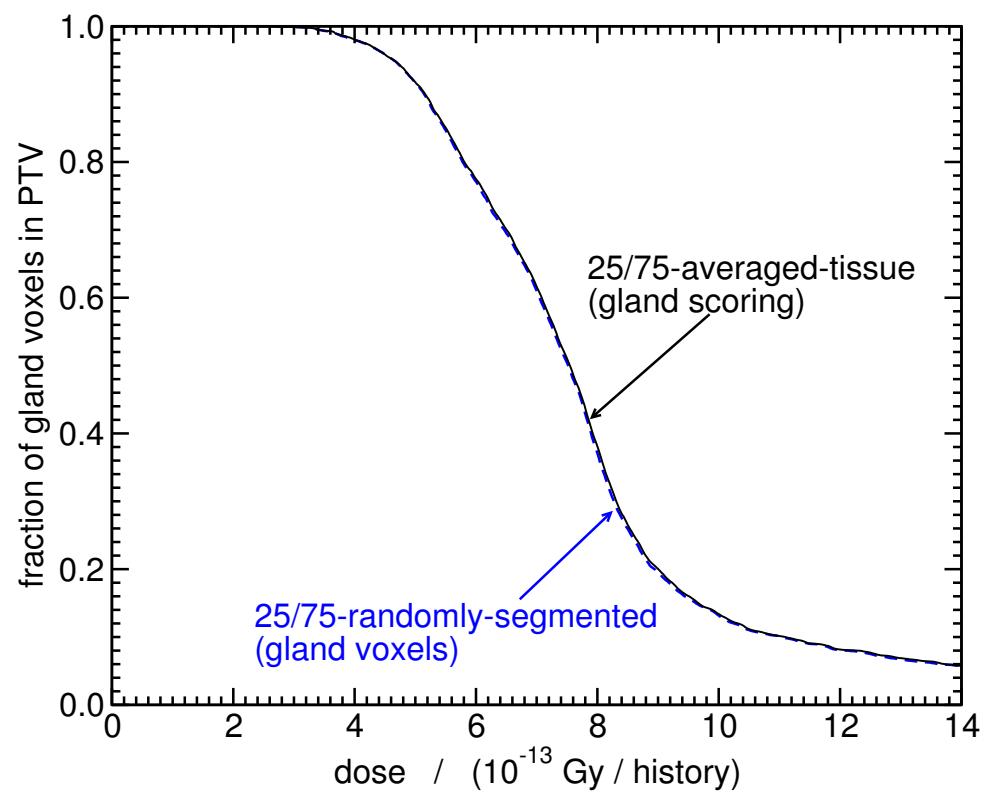

Figure 3.2: The DVHs for the dose to fibroglandular tissue in phantoms of $25 \%$ fibroglandular tissue and $75 \%$ adipose tissue by mass. The DVH for a $25 / 75$ randomly-segmented phantom where the volume considered consists only of those voxels which contain fibroglandular tissue is shown as well as the DVH for a 25/75-averaged-tissue phantom with dose scored in fibroglandular tissue where the volume considered is the same as that of the randomly segmented case.

tissue phantom (with dose scored in 25/75-averaged-tissue) and illustrates that differences in the shape of the DVH can arise when an averaged tissue is used to approximate the dose scored to segmented gland and adipose voxels. A small but clear difference is present between the two curves (similar to the results of Afsharpour et $\left.a l .{ }^{96}\right)$ which shows that, despite a nearly identical photon energy fluence for both calculations, inaccurate doses can occur when scoring in an averaged tissue.

The difference in shape between the two DVHs of figure 3.3 is explained by the differences in mass energy absorption coefficients (and will thus be sensitive to the choice of tissue composition). The mass energy absorption coefficient of gland is higher than that of 25/75-averaged-tissue and so, given the same photon energy fluence, those voxels containing gland will receive a higher dose and are seen in region A of the DVH. Conversely, adipose has a lower mass energy absorption coefficient 


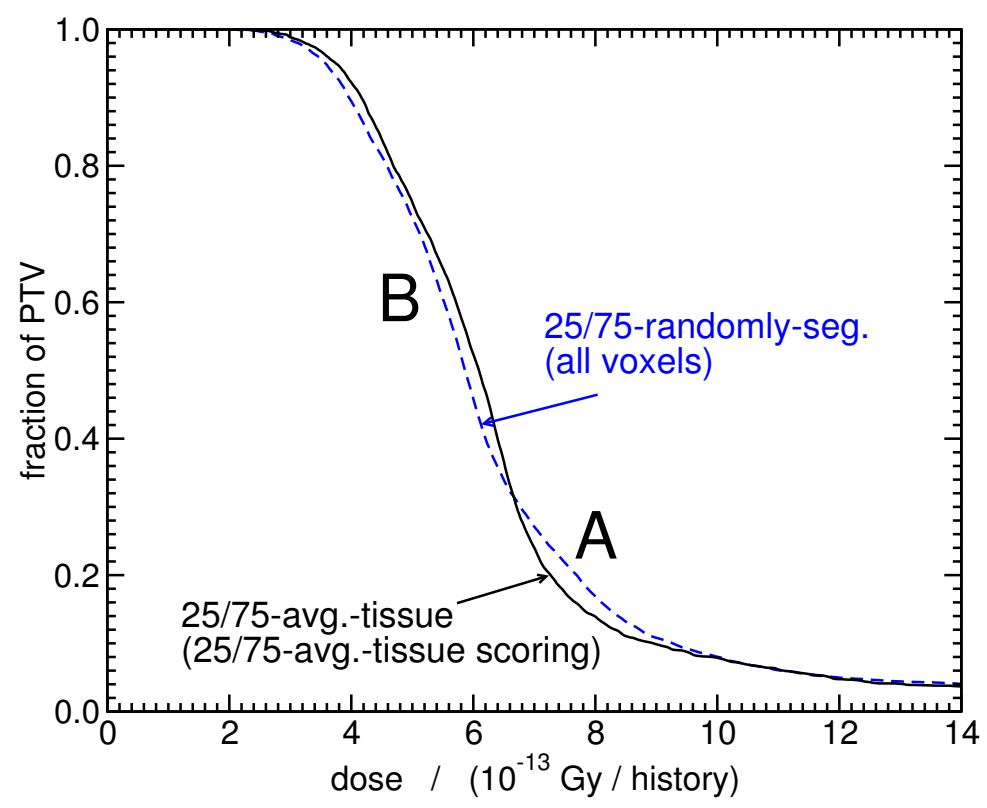

Figure 3.3: DVHs for the entire PTV in phantoms of $25 \%$ fibroglandular tissue and $75 \%$ adipose tissue by mass. Shown is the DVH for a 25/75-randomlysegmented phantom where the volume considered is the entire PTV (both adipose and fibroglandular tissue voxels with the dose scored to individual materials) as well as the DVH for a 25/75-averaged-tissue phantom with dose scored in the 25/75-averaged-tissue where the volume considered is also the entire PTV.

than 25/75-averaged-tissue and so absorbed dose will be lower for the same photon energy fluence as seen in region B in the DVH. These effects are illustrated in figure 3.4 which shows a dose profile in the $\mathrm{y}$-direction of the phantoms at $\mathrm{x}=0.2 \mathrm{~cm}$ and $\mathrm{z}=0.2 \mathrm{~cm}$. The calculated dose in the $25 / 75$-randomly-segmented phantom is higher or lower than that in the 25/75-averaged-tissue (25/75-averaged-tissue scoring) calculation depending on whether the voxel being considered is fibroglandular or adipose tissue. The 25/75-averaged-tissue (gland scoring) and 25/75-averaged-tissue (adipose scoring) results show agreement on the order of $0.5-1 \%$ with the $25 / 75$-randomlysegmented phantom when the voxel being considered is fibroglandular tissue or adipose tissue, respectively, which again is a result of the similar photon energy fluences in both phantoms. 


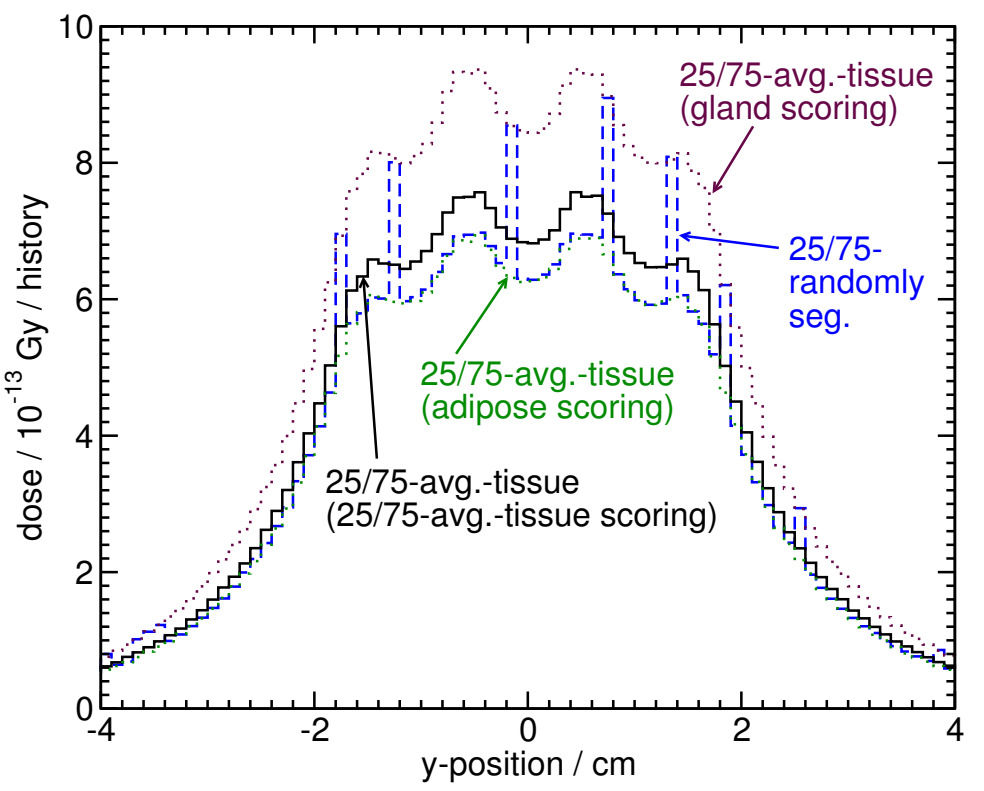

Figure 3.4: Dose vs. position along the $\mathrm{y}$-direction $(\mathrm{x}=0.2 \mathrm{~cm}, \mathrm{z}=0.2 \mathrm{~cm})$ for a $25 \%$ fibroglandular, $75 \%$ adipose tissue mixture by mass. The doses for 25/75-randomly-segmented, 25/75-averaged-tissue (25/75-averaged-tissue scoring), 25/75-averaged-tissue (adipose scoring) and 25/75-averaged-tissue (gland scoring) calculations are shown.

\subsubsection{Realistic segmentation and its effect on photon energy fluence}

Curves $I I$ and $I I I$ in figure 3.5 compare the DVHs for the dose to only the gland voxels within the PTV of the 26.4/73.6-realistically-segmented phantom (curve $I I$ ) to the dose to the voxels corresponding to the same spatial coordinates of the $26.4 / 73.6$ averaged-tissue phantom with dose scored in gland (curve $I I I$ ). It was observed in figure 3.2 that gland scoring resulted in DVHs having no noticeable difference between the 25/75-randomly-segmented and 25/75-averaged-tissue phantoms because on average the photon energy fluences are very close to each other. In figure 3.5 however, the lower gland dose in the 26.4/73.6-realistically-segmented phantom implies that the photon energy fluence within the fibroglandular tissue voxels is lower on average than in those same voxels in the 26.4/73.6-averaged-tissue phantom. This is 


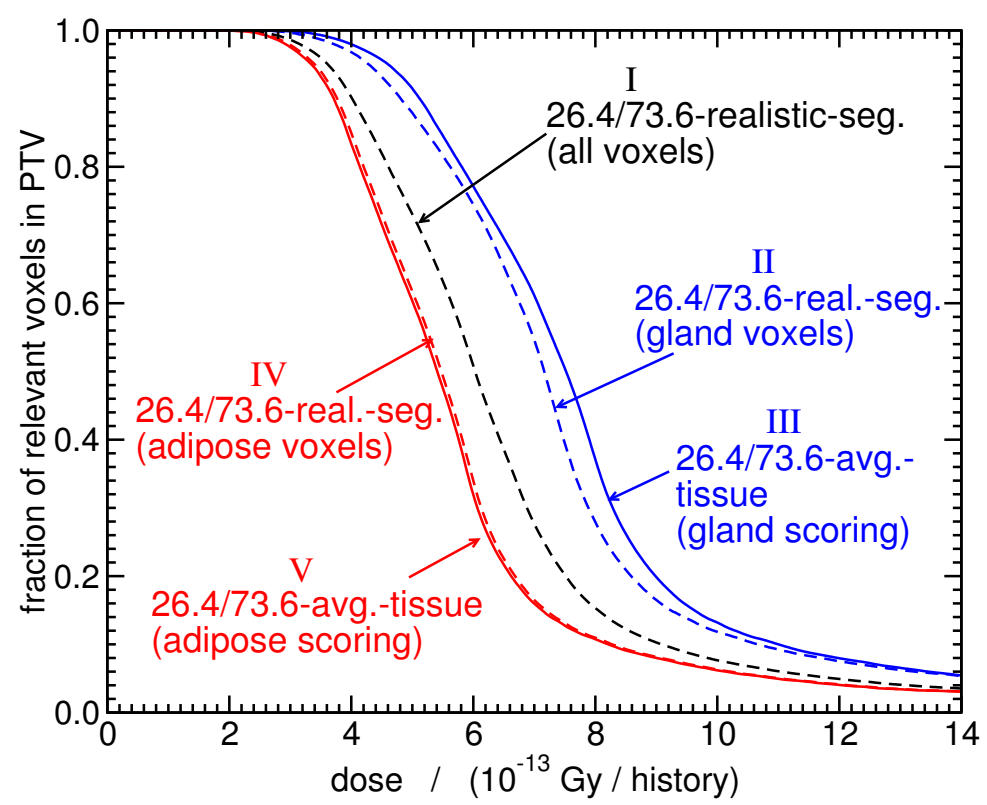

Figure 3.5: The DVHs for the dose to fibroglandular, adipose or all tissues in phantoms of $26.4 \%$ fibroglandular tissue and $73.6 \%$ adipose tissue by mass. Curve $I$ shows the dose to all voxels in a 26.4/73.6-realistically-segmented phantom. Curves $I I$ and $I I I$ show the dose to the gland voxels in a $26.4 / 73.6$-realisticallysegmented phantom and the dose to those same voxels in a 26.4/73.6-averagedtissue (gland scoring) phantom respectively. Curves $I V$ and $V$ show the dose to the adipose voxels in a 26.4/73.6-realistically-segmented phantom and the dose to those same voxels in a 26.4/73.6-averaged-tissue (adipose scoring) phantom respectively.

explained by the fact that a phantom with realistic segmentation has larger groupings of fibroglandular tissue voxels. The voxels towards the centre of these groupings are surrounded by other fibroglandular tissue voxels and photons delivering dose in these voxels will have experienced more attenuation compared to photons in the same voxels of an averaged tissue phantom as a result of the higher density and mass attenuation coefficient of gland. Hence, the dose is lower in these voxels due to reduced photon energy fluence.

Curve $I$ in figure 3.5 shows the DVH for the 26.4/73.6-realistically-segmented phantom where all the voxels are considered and dose is scored in the local medium. The large difference between curve $I$ and the one for only the gland voxels $(I I)$ 
illustrates the need to treat each tissue individually if the dose to a single tissue is of interest.

Curves $I V$ and $V$ in figure 3.5 compare the DVHs for the dose to only the adipose voxels within the PTV of the 26.4/73.6-realistically-segmented phantom $(I V)$ to the dose to the same voxels of the 26.4/73.6-averaged-tissue phantom with dose scored in adipose $(V)$. The larger groupings of adipose tissue (compared to a randomly segmented phantom) will cause the photon energy fluence in those voxels to be higher in a realistically segmented phantom than in the same voxels in an averaged tissue phantom. However, as the averaged tissue is $73.6 \%$ adipose by mass, the differences in photon energy fluence in the adipose tissue groupings will be less dramatic than in the case of gland tissue. This is reflected in the close agreement between curves $I V$ and $V$.

Figure 3.6 compares the DVHs for the dose to the entire PTV for the 26.4/73.6realistically-segmented phantom with the dose scored in each medium and the 26.4/73.6averaged-tissue phantom with dose scored in 26.4/73.6-averaged-tissue. While the differences between the DVHs are qualitatively similar to those of figure 3.3, they are smaller in magnitude. In this case, the change in photon energy fluence implied by figure 3.5 compensates to some degree for the change in scored dose as a result of scoring in gland and adipose versus averaged tissue (regions A and B in figure 3.3. . These competing effects combine to produce a calculation that provides more accurate dose volume histograms when considering the DVH for all PTV voxels.

Additionally, the differences seen in the comparison of curves $I I$ and $I I I$ in figure 3.5 reflect the inaccuracy of using the photon energy fluence in an averaged tissue to approximate the photon energy fluence in the gland voxels of a realistically segmented phantom. The gland voxels in a realistically segmented phantom will have a lower photon energy fluence on average than the same voxels of an averaged 


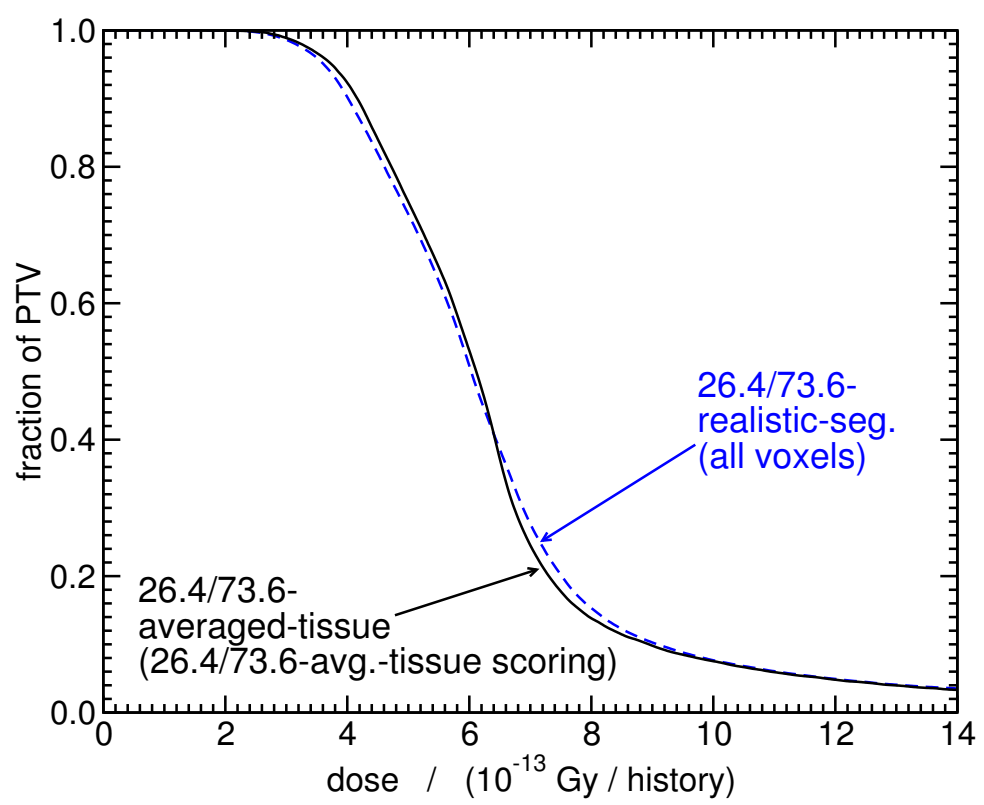

Figure 3.6: The DVHs for the entire PTV in a realistically segmented (26.4/73.6) phantom of $26.4 \%$ fibroglandular tissue and $76.3 \%$ adipose tissue by mass (same proportions as in figure 3.5 and same as curve $I$ in figure 3.5 and for a 26.4/73.6averaged-tissue phantom of the same proportions of tissue by mass with dose scored in the 26.4/73.6-averaged-tissue.

tissue phantom. This result is somewhat ameliorated in the comparison of the DVHs in figure 3.6 (where all voxels are considered) because considering both gland and adipose voxels of a realistically segmented phantom will mean that lower photon energy fluence in gland and higher photon energy fluence in adipose will both be included in the same volume. While photon energy fluence may differ significantly on a voxel by voxel basis, the average photon energy fluence in the entire PTV for an averaged tissue phantom will be closer to the average photon energy fluence in realistically segmented gland and adipose voxels than it will to the photon energy fluence in gland voxels alone. This is reflected as increased agreement in dose volume histograms.

In general, an averaged tissue phantom with individual voxel densities equal to that of a realistically segmented phantom will have a photon energy fluence in closer 
agreement with that of the realistically segmented phantom. However, as the densities of gland and adipose differ to a much smaller degree than the mass attenuation coefficients (approximately 7\% vs. 20\% difference respectively), the modeling of voxels of varying density does not fully overcome the differences in photon energy fluence. For example, in figure 3.5, the difference between the minimum dose that $90 \%$ of the volume receives (D90) for the 26.4/73.6-realistically-segmented (gland voxels) calculation and the 26.4/73.6-averaged-tissue (gland scoring) calculation is approximately 6.3\%. The use of a 26.4/73.6-averaged-tissue phantom with voxel densities equal to that of the 26.4/73.6-realistically-segmented phantom (not shown) only reduces the difference to $4.9 \%$.

It is important to note that the improvement in dose volume metrics that would result from improved simulation of photon energy fluence would only occur if one was considering the dose to either gland or adipose. The inaccuracy of using an averaged tissue phantom when the dose to gland is desired results from inaccurate photon energy fluence alone and so, any improvement in photon energy fluence will lead to improvement in dose volume metrics. In contrast, for dose to an entire volume (both gland and adipose), what accuracy exists in DVHs (figure 3.6 is a result of the competing effects of inaccurate photon energy fluence and inaccuracies from dose scored in averaged tissue rather than gland and adipose (regions $\mathrm{A}$ and $\mathrm{B}$ in figure 3.3. . Consequently, an improvement in photon energy fluence simulation will nullify the competing effect and the differences shown in figure 3.3 will resurface.

A second realistically segmented phantom was created using another computational phantom from Zastrow et al.100. This phantom was composed of approximately $55 \%$ fibroglandular and $45 \%$ adipose tissues. The effects found in figures 3.5 and 3.6 were qualitatively identical but with slightly differing magnitude with this denser phantom, confirming that the results of this work are not unique to the particular 
glandular density in the phantom used.

The results from the simulations with a seed configuration approximating a small, dense treatment (not presented) confirm the general effects and their relative magnitudes discussed above.

In all calculations, the choice of tissue compositions can significantly affect the dose calculated in the breast. For instance, the compositions in Hammerstein et al. $\underline{90}$ could be used instead of those of Woodard and White and this choice would affect the magnitude of the effects discussed in this work (e.g., the mass energy absorption coefficient for gland at $20 \mathrm{keV}$ is $17 \%$ lower for the composition in Woodard and White than that in Hammerstein et al. ${ }^{90}$ ) but would not change the conclusions.

\subsubsection{The effect of calcifications}

A 22.5/72.5/5-randomly-segmented phantom with $22.5 \%$ fibroglandular tissue, $72.5 \%$ adipose tissue and $5 \%$ calcification by mass was created. In figure 3.7, the DVH for the dose to the fibroglandular voxels within the PTV is compared to the DVH of the voxels corresponding to the same spatial coordinates for a 22.5/72.5/5-averaged-tissue phantom (gland scoring). The lower dose for the 22.5/72.5/5-randomly-segmented phantom shows that, despite the media being randomly distributed, calcifications in the breast significantly lower the average photon energy fluence.

Figure 3.8 contains DVHs calculated for the entire PTV. Curve $I$ shows the DVH for the entire PTV for the 22.5/72.5/5-randomly-segmented phantom. The disagreement between curve $I$ and the DVH for the 22.5/72.5/5-averaged-tissue phantom with dose scored in 22.5/72.5/5-averaged-tissue (curve $I I$ ) shows that the difference in photon energy fluence between the 22.5/72.5/5-randomly-segmented phantom and the 22.5/72.5/5-averaged-tissue phantom is too large to be compensated for by changes in 


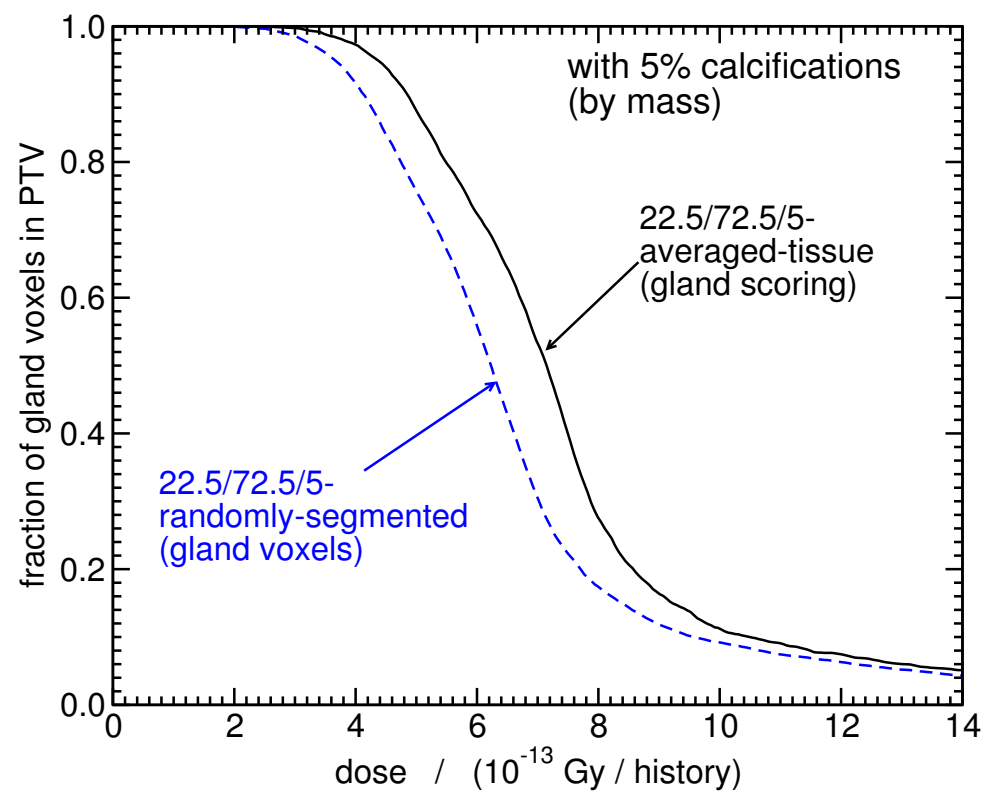

Figure 3.7: DVHs for phantoms composed of $22.5 \%$ fibroglandular tissue, $72.5 \%$ adipose tissue, and 5\% calcification by mass. Shown are the DVH calculated from a 22.5/72.5/5-randomly-segmented phantom (with $(1 \mathrm{~mm})^{3}$ calcifications) considering only those voxels containing fibroglandular tissue as well as the DVH from a 22.5/72.5/5-averaged-tissue (gland scoring) phantom with the same voxels considered as the 22.5/72.5/5-randomly-segmented phantom.

scored dose between gland, adipose, calcification and the 22.5/72.5/5-averaged-tissue of those three media. Curve $I I I$ is for calculations with a phantom that contains a 22.5/72.5/5-averaged-tissue of gland, adipose and calcification but with the density of each voxel assigned to the density of the same voxel in the randomly segmented phantom. The fact that curve $I I I$ closely agrees with curve $I I$ implies that the assignment of accurate voxel densities does not improve the ability of an averaged tissue phantom to approximate the photon energy fluence of a segmented phantom when calcifications are present. The inaccuracy in the photon energy fluence must result almost entirely from the inability of an averaged tissue to model the significant difference between the mass attenuation coefficients of gland and adipose versus calcifications.

Curve $I V$ of figure 3.8 shows the DVH calculated for a phantom containing 


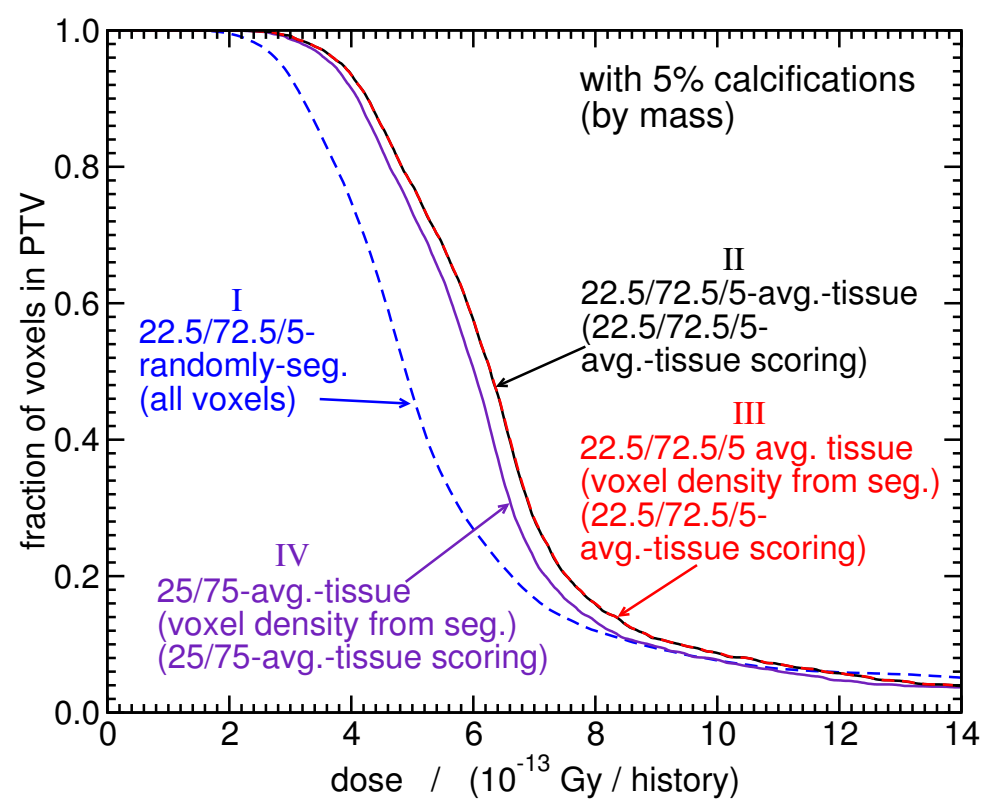

Figure 3.8: DVHs for phantoms composed of $22.5 \%$ fibroglandular tissue, $72.5 \%$ adipose tissue, and $5 \%$ calcification by mass. In all cases, DVHs are calculated considering all voxels in the PTV. Shown are the DVH calculated from a 22.5/72.5/5-randomly-segmented phantom (with $(1 \mathrm{~mm})^{3}$ calcifications) considering every voxel within the PTV $(I)$, the DVH from a $22.5 / 72.5 / 5$-averagedtissue $(22.5 / 72.5 / 5$-averaged-tissue scoring) phantom of a single averaged density (II), the DVH from a 22.5/72.5/5-averaged-tissue) (22.5/72.5/5-averaged-tissue scoring) phantom where voxel densities match those of the $22.5 / 72.5 / 5$-randomlysegmented phantom $(I I I)$, and a 25/75-averaged-tissue (25/75-averaged-tissue scoring) phantom where voxel densities are identical to those of curves $I$ and $I I I$ $(I V)$.

a 25/75-averaged-tissue composed of only gland and adipose where voxel densities are assigned to the density of the same voxel in the $22.5 / 72.5 / 5$-randomly-segmented phantom (curve I) (i.e., some voxels will have the density of calcification). This curve is used to approximate the result one would achieve if the presence of calcifications was not considered in the material composition of the breast but the density of the calcifications was taken from CT data. Although curve $I V$ significantly disagrees with the randomly segmented model, it is slightly closer than curves $I I$ and $I I I$. This shift to the left results from the decrease in mass energy absorption coefficient for 25/75-averaged-tissue when the calcifications are not included. 
Figure 3.9 illustrates the effect of considering the dose to only the gland and adipose in a phantom that contains calcifications. Curves $I$ and $I I$ are identical to curves $I$ and $I I$ of figure 3.8. Curve $I I I$ shows the DVH for only the gland and adipose voxels of the 22.5/72.5/5-randomly-segmented phantom. As the voxels containing calcification only correspond to $1.6 \%$ of total volume, the omission of the dose to the calcifications has little effect on the DVH. This differs from curve $I V$ which shows the DVH for the voxels corresonding the same spatial coordinates of a $22.5 / 72.5 / 5$ averaged-tissue (25/75-averaged-tissue scoring) phantom. In this calculation, photon transport occurs in an averaged tissue containing calcification but dose delivery in an averaged tissue without calcification. While the elimination of calcification (and its high $\mathrm{Z}$ contribution to composition) from the scoring averaged tissue moves curve $I V$ closer to agreement with curve $I I I$ (compared to $I I$ versus $I$ ), the inability of the 22.5/72.5/5-averaged-tissue phantom to approximate the photon energy fluence of the 22.5/72.5/5-randomly-segmented phantom is clear.

\subsection{Conclusions}

The photon energy fluence is not accurately determined if an averaged tissue phantom

is used in Monte Carlo simulations. Given the typical composition of the breast ${ }^{89}$, if the dose to gland tissue within the volume considered is desired, then the phantom must be properly segmented to achieve accurate doses and dose volume metrics. If dose to adipose tissue is desired, then an averaged tissue (adipose scoring) phantom may be sufficient to achieve accurate dose volume metrics. If dose metrics for the entire volume (all tissues) are desired and dose is scored in an averaged tissue, inaccuracies in the photon energy fluence will partially compensate for inaccuracies in scoring dose in an averaged tissue rather than in gland and adipose separately. While 


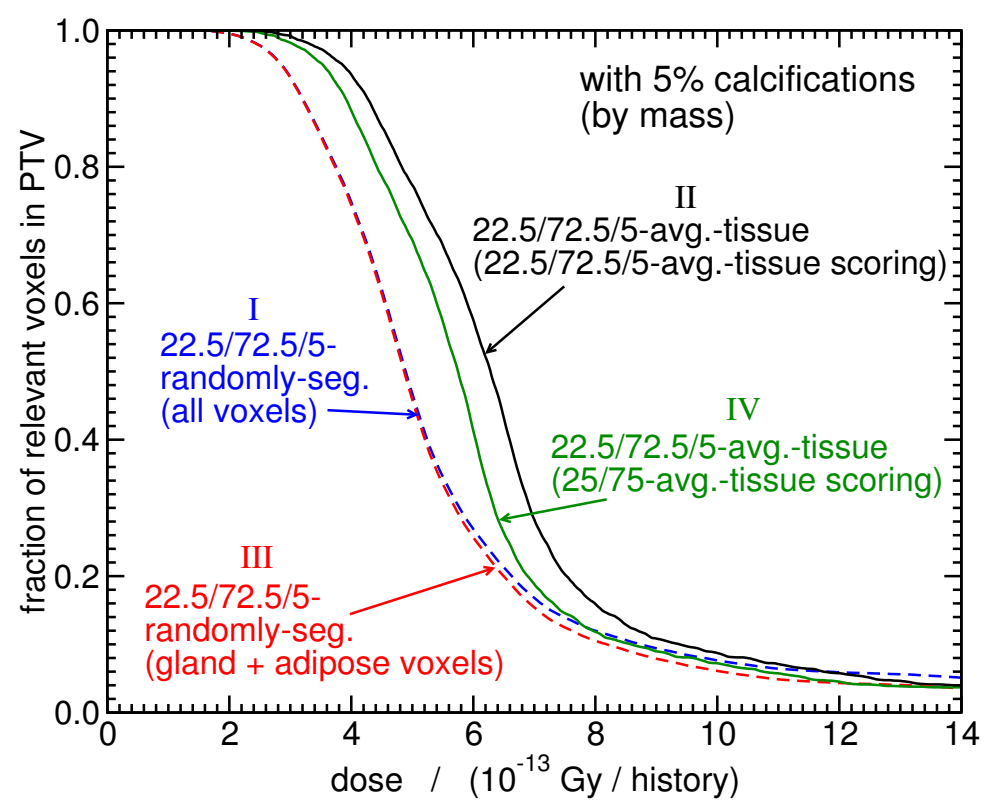

Figure 3.9: DVHs for phantoms composed of $22.5 \%$ fibroglandular tissue, $72.5 \%$ adipose tissue, and $5 \%$ calcification by mass. Curves $I$ and $I I$ show the DVHs for all the voxels in the PTV for the 22.5/72.5/5-randomly-segmented phantom and 22.5/72.5/5-averaged-tissue (22.5/72.5/5-averaged-tissue scoring) phantom respectively. Curve $I I I$ is the DVH for the gland and adipose voxels of the 22.5/72.5/5-randomly-segmented phantom and curve $I V$ is the $\mathrm{DVH}$ for the 22.5/72.5/5-averaged-tissue (25/75-averaged-tissue scoring) phantom.

significant dose differences may exist on a voxel by voxel basis, reasonably correct dose volume metrics will result.

Regardless of whether the dose to a single tissue or an entire volume is desired, the consideration of calcifications in a breast phantom necessitates the use of realistic segmentation as the effect of the composition of calcifications on the photon energy fluence is large.

Further work is required to extend the conclusions of the current work for ${ }^{103} \mathrm{Pd}$ to other low energy brachytherapy sources (e.g. ${ }^{125} \mathrm{I}$ and $50 \mathrm{kV}$ electronic brachytherapy sources). While the ratio of mass energy absorption coefficients of gland to adipose remain relatively constant in the energy range of interest, the ratio of mass attenuation coefficients of gland to adipose converge as energy increases, reaching approximately 
1.05 at $50 \mathrm{keV}$. It is expected that the observed effects are not as important for higher-energy sources such as ${ }^{192}$ Ir because the mass energy absorption and mass attenuation coefficients of the breast tissues are in much closer agreement at these photon energies.

The assignment of voxel densities based on CT data to an averaged tissue phantom reduces differences seen in photon energy fluence but not to such a degree that differences in dose volume metrics disappear.

It is likely that the methods commonly used in mammography radiation protection calculations overestimate the average dose to the glandular tissue. This arises because averaged tissue phantoms do not accurately reflect the photon energy fluence in the gland of a realistic breast. 


\section{Chapter 4}

\section{Exploring model-based dose calculations for ${ }^{125}$ I lung brachytherapy}

Although ${ }^{125}$ I lung brachytherapy has enjoyed considerable success, the standard practices for dosimetry and treatment planning involve significant approximations. Due to the clinical nature of the implant, the final placement of the seeds cannot be precisely predetermined and a simple nomogram of planar implant is used for pre-planning ${ }^{9}$. A seed activity look up table is used to determine the intended row spacing of the seeds to achieve approximately $100 \mathrm{~Gy}$ at a central point $5 \mathrm{~mm}$ from the plane of the implant. Post-treatment analysis typically involves computed tomography (CT) imaging and contouring the suture line and other volumes of interest. In addition, the seed positions are identified and dose calculation is performed according to the TG-43 formalism 25 , i.e., seeds in a homogeneous water environment and no interseed attenuation. Thus, clinical dose calculations for lung brachytherapy neglect the highly heterogeneous lung geometry. In reality, the patient treatment geometry involves lung and other tissues which differ in atomic composition and density from water. Considering the significant differences in mass energy absorption and mass attenuation coefficients of various tissues and water at the energies of photons emitted by ${ }^{125} \mathrm{I}$ seeds, it is expected that heterogeneities may significantly affect dosimetry for

lung brachytherapy ${ }^{24}$. While this was confirmed by a preliminary investigation $\sqrt{55}$, no 
calculations using voxelized patient data for permanent implant lung brachytherapy had been reported before the work presented in this chapter.

The focus of this chapter is the application of full Monte Carlo simulations of ${ }^{125}$ I lung brachytherapy, including considerations that must be addressed in order for patient CT data to be used in model-based dose calculation algorithms (MBDCA) for lung brachytherapy. While many of the considerations are applicable to low-energy brachytherapy in general and have been identified in the literature, changes in dose as a result of these considerations have been infrequently reported and, in the case of lung brachytherapy, have never been investigated.

In ${ }^{125} \mathrm{I}$ lung brachytherapy, CT scans are taken after implantation of ${ }^{125} \mathrm{I}$ seeds. The high-Z, high density nature of these seeds leads to streaking artifacts which, if uncorrected, may lead to large discrepancies between assigned and true voxel compositions and densities 50 . The correction of these CT artifacts presents a challenge due to the small size and significant number of seeds. Approaches to mitigating artifacts due to brachytherapy seeds remains an active area of research $\underline{50}+52$. Additionally, a naive use of CT data of small spatial dimensions may lead to a phantom model of insufficient extent to accurately model back-scattered photons and so padding of the CT data may be required.

Beyond ensuring the accuracy of the CT phantom, accurate assignment of material composition and density to each voxel can pose a challenge. A sophisticated tissue segmentation method - the stoichiometric method - has been introduced ${ }^{44}$; however, this method may not be feasible in all clinics and its accuracy for low-energy photon dose calculations is unknown. The degree of detail in the tissue assignment scheme (i.e., number and choice of tissues) may have a significant effect on the dose. Finally, the effect of errors in voxel density assignment on dose has not been studied. 
In this chapter, Monte Carlo calculations are performed with the EGSnrc usercode BrachyDose to determine dose distributions for six patients treated with intraoperative ${ }^{125} \mathrm{I}$ lung brachytherapy at the Mayo Clinic Rochester. Modelling considerations as well as their effect on patient dose distributions are studied. Additionally, doses calculated with the Monte Carlo technique are compared to those found via the TG-43 approach.

\subsection{Methods}

Six patients were treated for stage I non-small cell lung cancer with ${ }^{125} \mathrm{I}$ (GE Healthcare/Oncura, Arlington Heights IL USA, model 6711) mesh brachytherapy in conjunction with sublobar resection at the Mayo Clinic Rochester. Source air kerma strengths ranged from approximately $0.70 \mu \mathrm{Gy} \cdot \mathrm{m}^{2} / \mathrm{h}$ to $0.86 \mu \mathrm{Gy} \cdot \mathrm{m}^{2} / \mathrm{h}$. As per protocol Z4032 of the American College of Surgeons Oncology Group (2005), post-implant CT scans (GE LightSpeed RT 16) were taken within 30 days of implantation. In each of the anterior-posterior and lateral directions, the scans have 512 voxels with equal widths ranging from approximately $0.02 \mathrm{~cm}$ to $0.04 \mathrm{~cm}$ for total image widths of approximately $11 \mathrm{~cm}$ to $20 \mathrm{~cm}$. In the superior-inferior direction, the scans have a consistent voxel width of $0.0625 \mathrm{~cm}$ with the number of voxels ranging from 125 to 253 for a total image width ranging from $8 \mathrm{~cm}$ to $16 \mathrm{~cm}$. From the CT images, seed positions are determined, suture lines are contoured, and PTV structures are delineated from the suture lines: PTV05, PTV07, PTV10, and PTV20 are $5 \mathrm{~mm}, 7 \mathrm{~mm}, 10 \mathrm{~mm}$ and $20 \mathrm{~mm}$ expansions of the suture line, respectively.

Seeds are fully modelled ${ }^{77}$ and are superimposed on a voxelized geometry specified by EGSnrc phantom files (.egsphant). Unless otherwise stated, dose is scored in the local medium in each voxel. Simulations are performed with $2.7 \times 10^{10}$ histories to achieve average statistical uncertainties of at most $0.2 \%$ in PTV20 volumes. 
Simulations of fewer histories achieving less than $2 \%$ average statistical uncertainty in PTV05 volumes take less than 65 minutes on one $3.0 \mathrm{GHz}$ Woodcrest core for the small voxel sizes used (approximately $0.7 \mathrm{~mm} \times 0.7 \mathrm{~mm} \times 0.625 \mathrm{~mm}$ ); running calculations in parallel on a modest cluster of 30 cores would reduce calculations to a couple of minutes.

\subsubsection{Correcting metallic artifacts}

Patient CT data contain streaking artifacts which result from the presence of forty brachytherapy seeds in the treatment region. A number of methods are used to correct CT data for these metallic artifacts.

The first metallic artifact reduction method considered, referred to in the following as virtual sinogram metallic artifact correction and denoted "vsMAR", uses a filtered back-projection on a modified virtual sinogram and is an example of a projection completion method. This method, which employs a virtual sinogram, was introduced by Abdoli et al. ${ }^{103 / 104}$ for circumstances when raw sinogram data are

unavailable and is a variation of similar methods using raw sinogram data $49 \mid 105+107$ often denoted "MAR". In the MAR method, metallic objects in a CT image are segmented using a CT number threshold. A metal-only image is extracted and the image is forward projected to obtain a sinogram corresponding to metallic objects. The metal-only sinogram is used to delineate the bins of raw sinogram data that correspond to projections containing metallic artifacts. These bins are removed from the sinogram and replaced by an interpolation of the surrounding projections. The corrected sinogram is then reconstructed to produce an artifact-reduced image.

Raw sinogram data were unavailable for the patient CT data used in the present work and hence the vsMAR method is used ${ }^{103] 104}$. A virtual sinogram created by 
performing forward projection on the CT image is used in place of the raw sinogram. A threshold of 2000 Hounsfield units (HU) is used to identify metallic objects and the projections passing through these objects are identified and deleted from the virtual sinogram. A spline-interpolation is used to refill deleted portions of the virtual sinogram. Built-in Radon transforms and inverse Radon transforms with a ramp filter are used in MATLAB (The Mathworks, Natick Mass. USA) for forward and back projections, respectively; 180 projections were used over 180 degrees. While CT numbers in images undergoing Radon and inverse Radon transforms cannot be perfectly preserved, differences are less than image noise. Regions of interest drawn in homogeneous areas (tissue, adpiose, etc. outside of areas affected by metallic artifacts) see mean CT numbers that differ by less than $15 \mathrm{HU}$ with equal standard deviations of approximately $30 \mathrm{HU}$. Dose metrics calculated using a phantom generated after artificially shifting CT numbers by $15 \mathrm{HU}$ differ by less than $1 \%$ (and in most cases by less than $0.5 \%$ ) from those computed with the original phantom.

With the vsMAR method, the streak artifacts throughout the CT image are reduced to the same magnitude as the noise elsewhere in the image. Figure 4.1 shows a line profile of the CT numbers for a corrected and uncorrected image. The profile passes through metallic streak artifacts in the vicinity of ${ }^{125} \mathrm{I}$ seeds. An example of a portion of an uncorrected and vsMAR corrected image can be seen in figures 4.2 and $b$, respectively.

In projection completion methods of artifact reduction, two challenges arise: accurately determining projections that pass through metallic objects and correction of these projections. When only virtual sinograms are available, the surrounding projections used to correct deleted projections corresponding to metal will themselves have been affected by the artifacts. Consequently, the interpolated values will remain somewhat corrupted (if less so) and resulting CT numbers in the immediate vicinity 


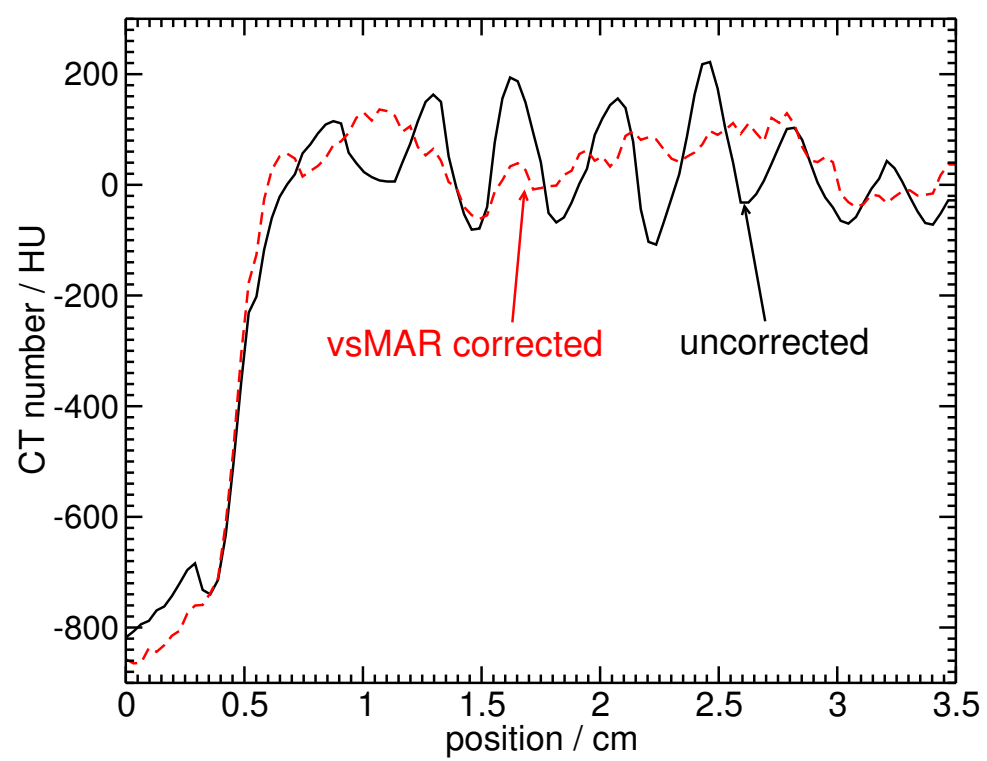

Figure 4.1: CT profiles for uncorrected and vsMAR corrected data for patient 5. The profile passes from lung to soft tissue through metallic artifact streaks near a seed.

of the seeds will be higher than they would if the original raw sinogram data were available for interpolation. In the present work, these high $\mathrm{CT}$ numbers result in the assignment of bony media to the affected voxels. As BrachyDose models the ${ }^{125} \mathrm{I}$ seeds separately from the voxelized patient geometry, the resulting model may include seeds surrounded by bony media. Consequently, a second metallic artifact reduction method, "simple threshold replacement" (STR), is considered. In this method, a $1 \mathrm{~cm}$ cube is defined about each seed position. Within each cube, if the CT number lies above a certain threshold, it is reassigned to a lower number. The threshold is selected to be $88 \mathrm{HU}$ so that for all tissue assignment schemes employed (Fig. 4.4 and section 5.1.2, no cartilage or bony media are modelled in these regions. This is reasonable on anatomic grounds: visual inspection of the CT images confirms that there is no bone in these regions. Unless otherwise stated, CT numbers above the threshold value of $88 \mathrm{HU}$ are replaced with $-200 \mathrm{HU}$, corresponding to the most dense lung tissue. A replacement value of $0 \mathrm{HU}$ is also used for several simulations to compare the effect 


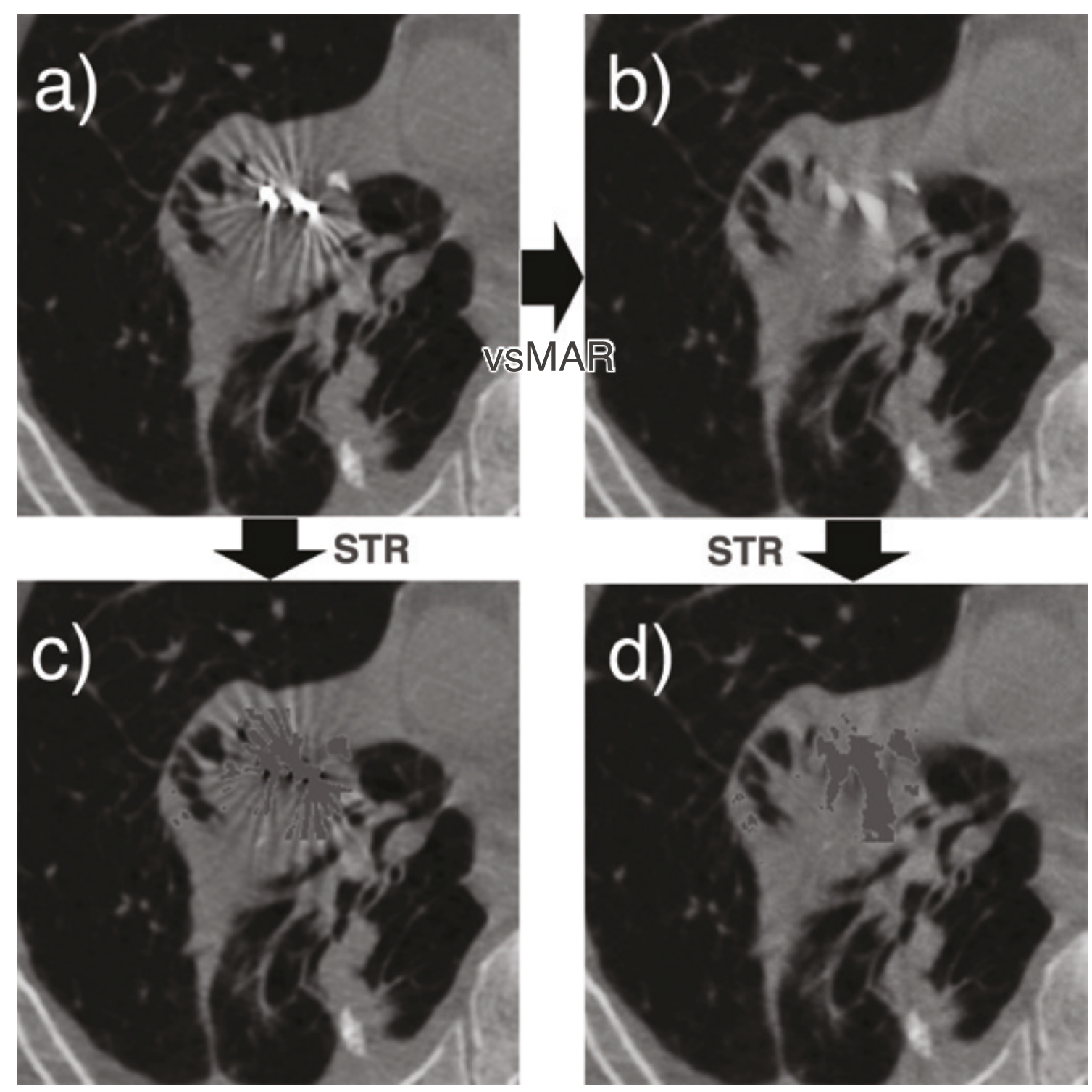

Figure 4.2: A portion of a CT image showing (a) no artifact correction, (b) metallic artifact correction (vsMAR), (c) simple threshold replacement on an uncorrected image (STR), and (d) simple threshold replacement on a metallic artifact corrected image (vsMAR+STR).

of different replacement values on the dose.

Phantoms are created with the STR correction alone, as well as the vsMAR correction followed by the STR correction (denoted "vsMAR+STR"). Figures 4.2. and d show examples of the STR method applied to uncorrected and vsMAR corrected CT data, respectively. 
Calculations to compare metallic artifact correction schemes are performed using the simple tissue assignment scheme, described in the following section.

\subsubsection{Tissue assignment schemes}

Before CT phantoms are converted to EGSnrc phantom files (.egsphant), the voxels are resized and resampled such that the maximum number of voxels in any direction is 240 for computational efficiency. This results in phantom voxels with dimensions of approximately $0.07 \mathrm{~cm} \times 0.07 \mathrm{~cm} \times 0.0625 \mathrm{~cm}$. Phantoms are created in which voxel elemental composition and mass densities are determined by the $\mathrm{CT}$ numbers of the corresponding voxels in the CT phantoms.

Tissue compositions taken from Woodard and White ${ }^{\sqrt[85]{5}}$ and White et $_{\text {al. }}^{\left[{ }^{86}\right.}$ (also listed in ICRU report 46 (Ref. 87)) are given in table 2.1 (page 19). The mass attenuation and energy absorption coefficients for the media used in this chapter for the mean emitted photon energy of ${ }^{125} \mathrm{I}(28 \mathrm{keV})$ can be seen in table 4.1. The density of each voxel is determined by the experimentally derived CT number to mass density calibration in table 4.2 .

In this study, the use of a stoichiometric calibration or other simpler tissue calibrations was unavailable and so the tissue assignment schemes used represent a com-

promise between those listed in the literature $44|45| 47$ and the features that could be distinguished from the frequency distribution of CT numbers in the phantoms (see figure 4.3). 
Table 4.1: Mass attenuation (from $\mathrm{XCOM}^{[30}$ ) and mass energy absorption coefficients (calculated with EGSnrc user-code g using XCOM) for $28 \mathrm{keV}$ photons.

\begin{tabular}{ccc}
\hline \hline Material & $\begin{array}{c}\mu / \rho(28 \mathrm{keV}) \\
\left(\mathrm{cm}^{2} / \mathrm{g}\right)\end{array}$ & $\begin{array}{c}\mu_{e n} / \rho(28 \mathrm{keV}) \\
\left(\mathrm{cm}^{2} / \mathrm{g}\right)\end{array}$ \\
\hline Adipose 1 & 0.360 & 0.127 \\
Adipose 2 & 0.348 & 0.116 \\
Adipose 3 & 0.348 & 0.105 \\
Air (TG-43) & 0.414 & 0.189 \\
Cartilage & 0.485 & 0.242 \\
Cortical bone & 1.617 & 1.317 \\
Heart 1 & 0.431 & 0.191 \\
Heart 3 & 0.443 & 0.200 \\
Heart & 0.446 & 0.204 \\
Lung & 0.443 & 0.201 \\
Muscle 1 & 0.435 & 0.194 \\
Muscle 2 & 0.439 & 0.198 \\
Muscle 3 & 0.444 & 0.203 \\
Red marrow & 0.397 & 0.161 \\
Ribs (2nd, 6th) & 1.104 & 0.830 \\
Ribs (10th) & 1.243 & 0.962 \\
Skin 1 & 0.414 & 0.176 \\
Skin 2 & 0.420 & 0.181 \\
Skin 3 & 0.427 & 0.187 \\
Water & 0.436 & 0.192 \\
Yellow Marrow & 0.341 & 0.110 \\
\hline \hline
\end{tabular}


Table 4.2: CT number / mass density calibration data.

\begin{tabular}{cc}
\hline \hline CT number $(\mathrm{HU})$ & mass density $\left(\mathrm{g} / \mathrm{cm}^{3}\right)$ \\
\hline-1000 & 0.00111 \\
-992 & 0.00121 \\
-976 & 0.00131 \\
-480 & 0.5 \\
-96 & 0.95 \\
0 & 1 \\
48 & 1.05 \\
128 & 1.1 \\
528 & 1.334 \\
976 & 1.603 \\
1488 & 1.85 \\
1824 & 2.1 \\
2224 & 2.4 \\
2640 & 2.7 \\
2832 & 2.83 \\
\hline
\end{tabular}

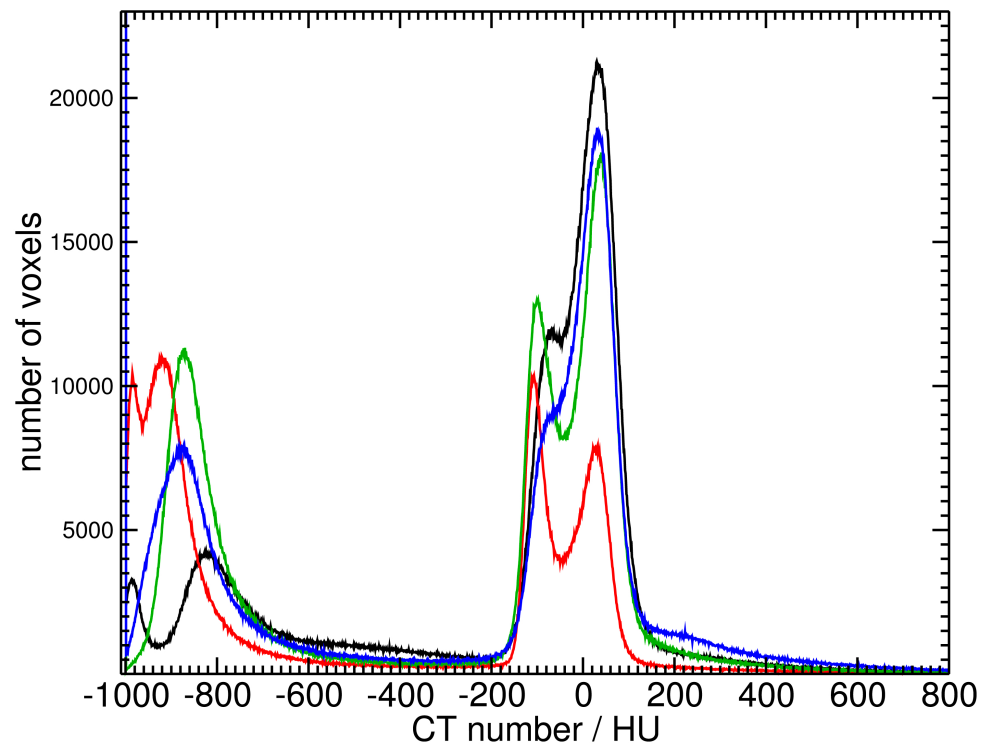

Figure 4.3: Histogram showing the frequency of CT numbers in several patient $\mathrm{CT}$ phantoms. Of note is the separation of peaks at $-50 \mathrm{HU}$, likely distinguishing adipose tissue from other soft tissues. Data from four of six patients are shown for readability and CT numbers above 800 (corresponding to bones and seeds) are not shown since they are relatively infrequent. 
Three tissue assignment schemes of varying complexity are used and can be seen in figure 4.4. The first scheme, denoted "detailed", contains 20 media and includes all tissues that may possibly be found in the CT images. Because each voxel is assigned a tissue based on its CT number, this scheme will result in tissues in nonsensical locations (eg. marrow outside of bones) and so illustrates the naive application of detailed tissue assignment. The second assignment scheme, denoted "moderate", contains 11 media and represents a more anatomically realistic, but still fairly detailed assignment. The three adipose tissues, each covering a reasonably large range of CT numbers, remain separate, while other soft tissues are now assigned to muscle 2 or heart - bloodfilled. The last scheme, denoted "simple", contains only 5 media and represents the traditional method of restricting the number of tissues to only a few. The comparison of these three schemes is used to study the differences in dose and dose metrics that occur with varying levels of tissue specificity. The differences in dose between phantoms created with these schemes also provide an estimate of the error that can be introduced by poor CT number to tissue conversion.

To investigate the result of an inaccurate CT number to mass density calibration, phantoms are created that have the same tissue assignment but densities determined by a calibration that is shifted by $\pm 50 \mathrm{HU}$.

As the CT data do not cover the full extent of the patient, $10 \mathrm{~cm}$ of water is added to every side of each phantom to ensure that the phantom extent is sufficient to properly model photon backscatter. However, comparisons with phantoms that lack this water padding indicate that this may not be necessary as local disagreement only exceeds $1 \%$ within approximately $3 \mathrm{~cm}$ of phantom boundaries. The largest volume considered, PTV20, does not approach this region in any of the six patients and the dose volume histograms are virtually identical for padded and non-padded phantoms. Tissue assignment schemes are compared using phantoms that have received 


\begin{tabular}{|c|c|c|c|}
\hline $\begin{array}{c}\text { CT } \\
\text { number }\end{array}$ & $\begin{array}{c}\text { detailed } \\
\text { air }\end{array}$ & $\begin{array}{c}\text { moderate } \\
\text { air }\end{array}$ & $\begin{array}{c}\text { simple } \\
\text { air }\end{array}$ \\
\hline$-950 /-949$ & lung - blood filled & lung - blood filled & lung - blood filled \\
\hline$-200 /-199$ & adipose 3 & adipose 3 & \multirow{3}{*}{ adipose 2} \\
\hline \multirow{2}{*}{$\begin{array}{l}-88 /-87 \\
-66 /-65\end{array}$} & adipose 2 & adipose 2 & \\
\hline & adipose 1 & adipose 1 & \\
\hline$-511-50$ & yellow marrow & \multirow{5}{*}{ muscle 2} & \multirow{11}{*}{ muscle 2} \\
\hline$-19 /-18$ & red marrow & & \\
\hline 26/27 & muscle 1 & & \\
\hline $\begin{array}{l}40 / 41 \\
42 / 43\end{array}$ & heart 1 & & \\
\hline $43 / 44$ & muscle 2 & & \\
\hline $44 / 45$ & heart 3 & \multirow{5}{*}{ heart - blood filled } & \\
\hline & heart - blood filled & & \\
\hline \multirow{3}{*}{$\begin{array}{l}73 / 74 \\
75 / 76\end{array}$} & skin 1 & & \\
\hline & skin 2 & & \\
\hline & skin 3 & & \\
\hline $89 / 90$ & cartilage & cartilage & \\
\hline $300 / 301$ & ribs (2nd, 6th) & ribs (2nd, 6th) & \multirow{3}{*}{ cortical bone } \\
\hline $750 / 751$ & ribs (10th) & ribs (10th) & \\
\hline $1183 / 1184$ & cortingl han & cortical hone & \\
\hline
\end{tabular}

Figure 4.4: Three tissue assignment schemes used. Row heights not to scale. CT number boundaries are indicated (eg. for detailed scheme, yellow marrow includes CT numbers -50 to -19$)$.

vsMAR+STR correction.

\subsubsection{Comparison with TG-43}

For each patient, simulations are repeated in a phantom in which all voxels are water (density of $0.998 \mathrm{~g} / \mathrm{cm}^{3}$ ) with no interseed attenuation (denoted TG43-sim). To es- 
timate dose differences between TG-43 calculations and heterogeneous Monte Carlo calculations, the TG43-sim doses are compared to those from simulations performed in phantoms obtained with the vsMAR+STR correction method and the simple tissue assignment scheme. In addition to simulations with these phantoms in which dose to the local tissue composing each voxel is scored (dose to medium in medium,

$\left.\mathrm{D}_{m, m}\right)$, these phantoms are also used to calculate collision kerma to water in medium which approximates dose to water voxels in medium, $\mathrm{D}_{w, m}$. This quantity is calculated by simulating radiation transport in the heterogeneous, non-water phantom (vsMAR+STR corrected with simple tissue assignment scheme) and using mass energy absorption coefficients for water in place of those for the local tissue (and thus energy deposition in water is calculated). These calculations of $\mathrm{D}_{m, m}$ and $\mathrm{D}_{w, m}$ permit investigation of the relative impacts of photon attenuation and energy deposition in non-water media compared to TG-43.

\subsection{Results}

\subsubsection{Correcting metallic artifacts}

The various metallic artifact reduction schemes considered affect the dose in a number of ways. This is illustrated in figure 4.5 which shows the dose profiles along a line passing through metallic artifact streaks in the vicinity of the seeds in patient 5 for the different metallic artifact correction schemes. The CT numbers for the uncorrected image along this line are also shown in the plot. Figure 4.6 shows the profile line superimposed on the uncorrected CT phantom. 


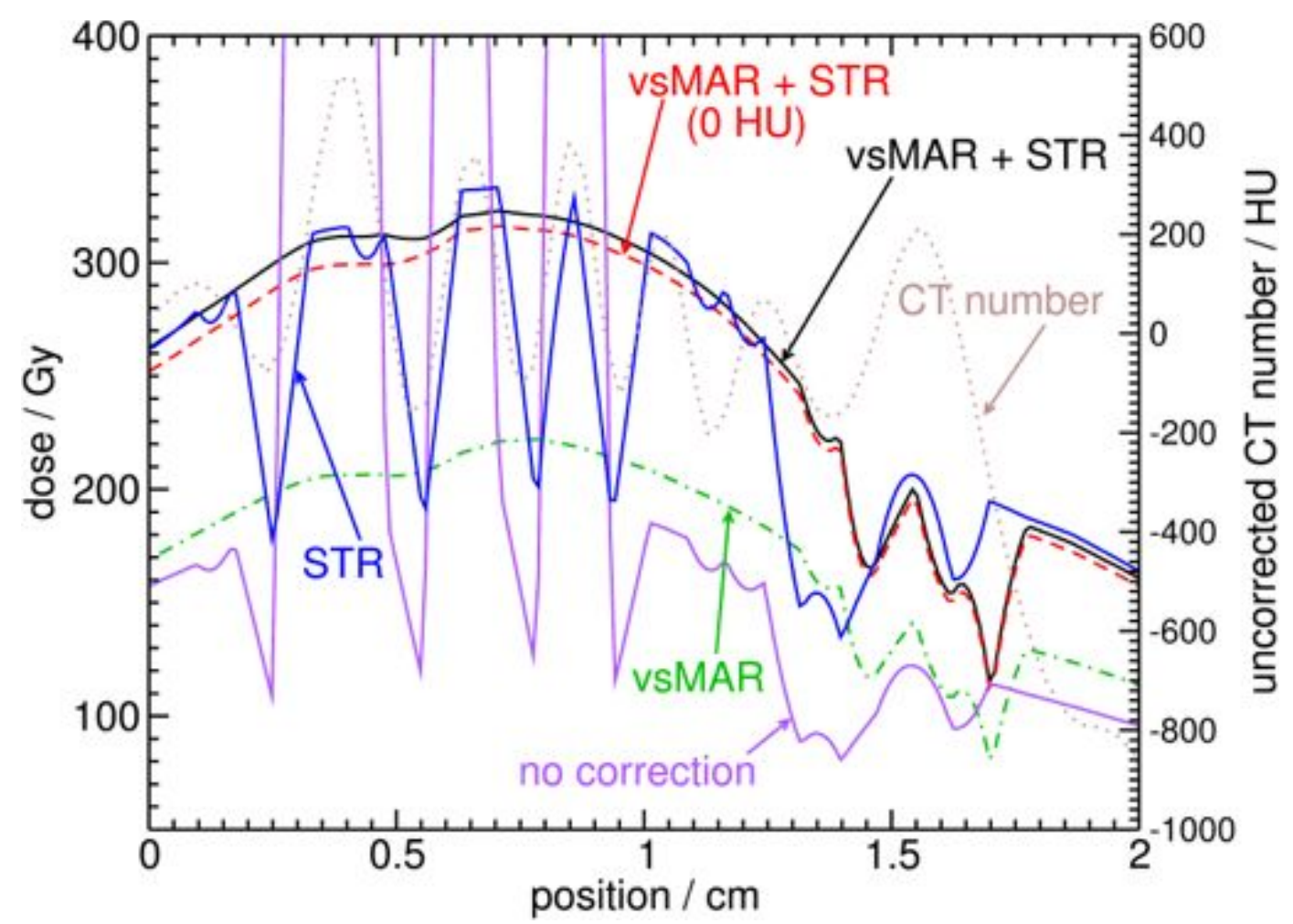

Figure 4.5: Dose (left axis) and uncorrected CT number (right axis) profile for patient 5 for calculations with the simple tissue assignment scheme applied to phantoms with vsMAR + STR correction, vsMAR + STR (with replacement value of $0 \mathrm{HU}$ ), STR correction alone, vsMAR correction alone, and no correction. The horizontal axis gives the position along the line shown in figure 4.6 .

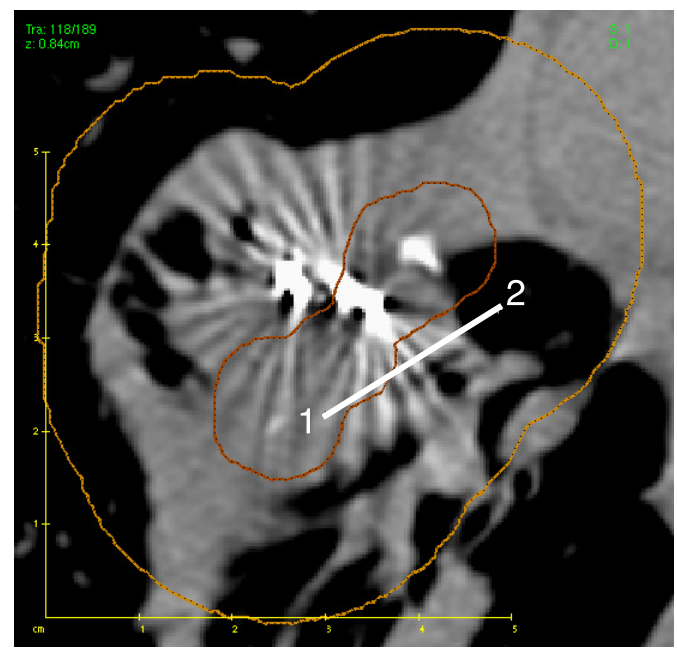

Figure 4.6: CT image for patient 5 showing the $\sim 2 \mathrm{~cm}$ line (going from 1 to 2 ) used for the profiles in figure 4.5. Outlines for PTV05 and PTV20 are also shown. 
The phantom with no correction has large spikes in dose where the CT numbers are high enough that the voxels are assigned to cortical bone regardless of tissue assignment scheme. Cortical bone has a high mass energy absorption coefficient: 1.317 $\mathrm{cm}^{2} / \mathrm{g}$ for $28 \mathrm{keV}$ photons versus $0.201 \mathrm{~cm}^{2} / \mathrm{g}$ and $0.198 \mathrm{~cm}^{2} / \mathrm{g}$ for lung and muscle-2, respectively. Additionally, in this uncorrected phantom, the voxels surrounding the seeds are modelled as bone, resulting in significant attenuation of emitted photon fluence. The dose is thus lower elsewhere in the phantom compared with all other phantoms considered. In figure 4.5, this is illustrated in lower doses in voxels with CT numbers below those of bone.

In the vsMAR corrected phantom, the streak artifacts are greatly reduced (see figure $4.2 \mathrm{~b}$, page 49 and so the dose profile of figure 4.5 no longer passes through bone. However, the region immediately surrounding the seeds is still modelled as bone but now having a lower density. So while there are now no dose spikes and the dose has increased compared to the uncorrected phantom, the dose is still lower than that obtained by other metallic artifact reduction methods due to the attenuation of emitted photons.

Applying the STR correction to the vsMAR corrected phantom (vsMAR+STR) decreases the photon attenuation significantly as the seeds are no longer surrounded by low density bone but are surrounded by lung tissue. Since the photon attenuation is decreased, the dose increases. It is noteworthy that the dose profiles for phantoms with replacement values of $-200 \mathrm{HU}$ (lung) and $0 \mathrm{HU}$ (muscle) differ by only a small amount and reflect only the difference in upstream attenuation between lung and denser muscle.

For the phantom with only the STR correction, the dose alternates between high and low; higher in regions where the CT numbers have been replaced with $200 \mathrm{HU}$ (lung) and lower in regions where the CT number has not been replaced 
and is mostly in the range of adipose which has a significantly lower mass energy absorption coefficient $\left(0.116 \mathrm{~cm}^{2} / \mathrm{g}\right.$ for $28 \mathrm{keV}$ photons versus $0.201 \mathrm{~cm}^{2} / \mathrm{g}$ for lung). The implications of the presence of adipose and muscle in the vicinity of the seeds are discussed in section 4.3 ,

Table 4.3 shows the volume that receives at least the prescription dose of 100 Gy $\left(\mathrm{V}_{100}\right)$ and the minimum dose that $90 \%$ of the volume receives $\left(\mathrm{D}_{90}\right)$ for PTV05, as well as $\mathrm{D}_{90}$ for PTV20 for all six patients for the metallic artifact correction methods discussed above. The percent difference with vsMAR+STR is also shown. As expected from figure 4.5, the vsMAR corrected phantoms and the uncorrected phantoms have significantly lower doses than the vsMAR+STR corrected phantoms. Interestingly, there is generally good agreement (differences are less than $2 \%$ ) between the vsMAR+STR corrected phantoms and the phantoms receiving only STR correction despite the reduction of streaks with the application of vsMAR. This is due to the fact that while significant dose differences occur in the regions of metallic artifacts, the majority of the voxels in the PTV05 and PTV20 volumes have similar or even equal assignments of material density and composition for the vsMAR+STR and STR correction methods. As long as the photon fluences in the vicinity of the seeds for the phantoms reasonably agree, the dose metrics will not differ significantly. This is further confirmed by figure 4.7 which shows dose volume histograms for patient 1 phantoms with various correction methods for PTV05 and PTV20.

Dose metrics for phantoms with vsMAR+STR that use an STR replacement value of $0 \mathrm{HU}$ (not shown) agree with those with vsMAR+STR that use an STR replacement value of $-200 \mathrm{HU}$ within approximately $2 \%$. 
Table 4.3: $\mathrm{V}_{100}$ and $\mathrm{D}_{90}$ for PTV05 and $\mathrm{D}_{90}$ for PTV20 for various artifact correction methods. For vsMAR, STR, and no corrections, the percent differences with vsMAR + STR correction are shown.

\begin{tabular}{|c|c|c|c|c|}
\hline Patient & vsMAR+STR & vsMAR & STR & none \\
\hline & \multicolumn{4}{|c|}{$\mathrm{V}_{100}$ (volume fraction) for PTV05 } \\
\hline 1 & 0.634 & $0.542(-14.6 \%)$ & $0.636(0.3 \%)$ & $0.451(-28.9 \%)$ \\
\hline 2 & 0.798 & $0.717(-10.2 \%)$ & $0.798(-0.1 \%)$ & $0.668(-16.3 \%)$ \\
\hline 3 & 0.736 & $0.670(-9.0 \%)$ & $0.734(-0.2 \%)$ & $0.602(-18.2 \%)$ \\
\hline 4 & 0.783 & $0.685(-12.4 \%)$ & $0.781(-0.3 \%)$ & $0.628(-19.8 \%)$ \\
\hline 5 & 0.941 & $0.853(-9.3 \%)$ & $0.940(-0.9 \%)$ & $0.801(-14.9 \%)$ \\
\hline \multirow[t]{2}{*}{6} & 0.921 & $0.731(-20.6 \%)$ & $0.916(-0.6 \%)$ & $0.633(-31.2 \%)$ \\
\hline & \multicolumn{4}{|c|}{$\mathrm{D}_{90}(\mathrm{~Gy})$ for PTV05 } \\
\hline 1 & 59.2 & $51.3(-13.3 \%)$ & $59.2(-0.0 \%)$ & $45.4(-23.4 \%)$ \\
\hline 2 & 64.4 & $46.9(-27.2 \%)$ & $63.5(-1.5 \%)$ & $41.0(-36.3 \%)$ \\
\hline 3 & 41.0 & $33.6(-18.1 \%)$ & $41.1(0.4 \%)$ & $30.0(-26.7 \%)$ \\
\hline 4 & 50.7 & $32.8(-35.3 \%)$ & $50.3(-0.8 \%)$ & $28.7(-43.4 \%)$ \\
\hline 5 & 130.2 & $85.3(-34.5 \%)$ & $128.5(-1.3 \%)$ & $74.5(-42.8 \%)$ \\
\hline \multirow[t]{2}{*}{6} & 107.3 & $77.8(-27.5 \%)$ & $105.6(-1.6 \%)$ & $69.8(-34.9 \%)$ \\
\hline & \multicolumn{4}{|c|}{$\mathrm{D}_{90}(\mathrm{~Gy})$ for PTV20 } \\
\hline 1 & 20.7 & $18.1(-12.6 \%)$ & $20.8(0.7 \%)$ & $16.0(-22.5 \%)$ \\
\hline 2 & 28.1 & $21.7(-22.9 \%)$ & $28.5(1.2 \%)$ & $19.2(-31.8 \%)$ \\
\hline 3 & 16.3 & $13.8(-15.3 \%)$ & $16.2(-0.6 \%)$ & $12.1(-26.0 \%)$ \\
\hline 4 & 17.9 & $11.9(-33.5 \%)$ & $18.1(1.1 \%)$ & $10.6(-41.0 \%)$ \\
\hline 5 & 31.3 & $21.9(-30.2 \%)$ & $31.6(0.8 \%)$ & $19.5(-37.7 \%)$ \\
\hline 6 & 22.9 & $16.5(-27.9 \%)$ & $23.1(0.9 \%)$ & $15.3(-33.2 \%)$ \\
\hline
\end{tabular}

\subsubsection{Tissue assignment schemes}

Table 4.4 shows $\mathrm{V}_{100}$ and $\mathrm{D}_{90}$ for PTV05, as well as $\mathrm{D}_{90}$ for PTV20 for all six patients using the simple, moderate, and detailed tissue assignment schemes. In all cases, phantoms that received the vsMAR+STR correction are used. Also shown is the percent difference in metrics of moderate and detailed schemes to the simple scheme. Example dose volume histograms for PTV05 and PTV20 for patient 3 using these tissue assignment schemes can be seen in figure 4.8 .

The greatest difference in dose metrics is for patient 6 with an $8.0 \%$ difference in $\mathrm{D}_{90}$ between the simple and detailed schemes. In general, the moderate scheme 


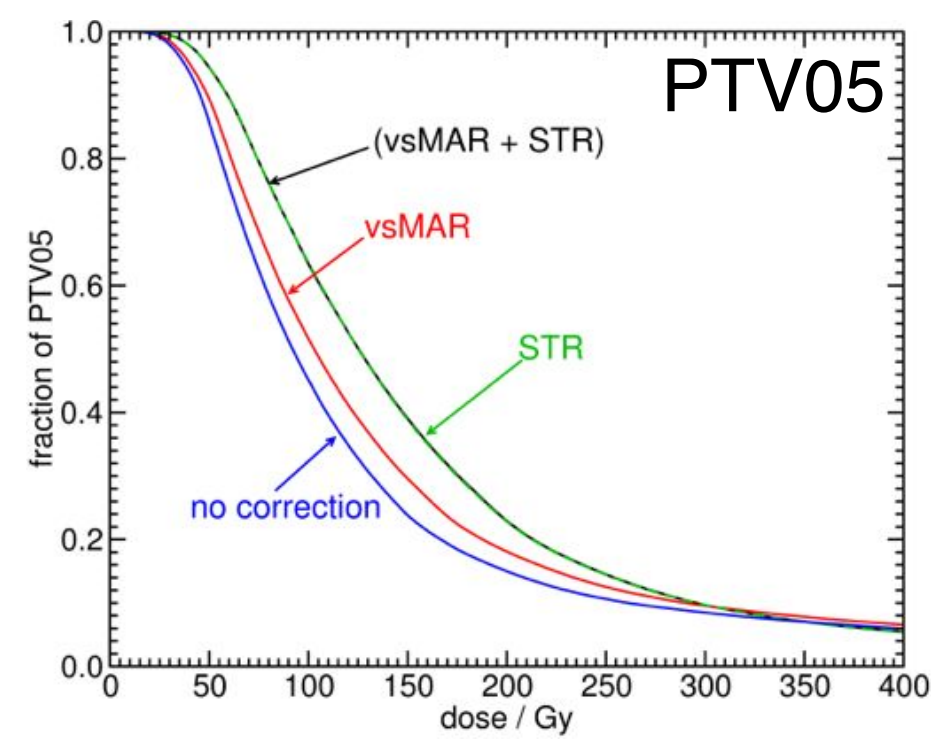

(a)

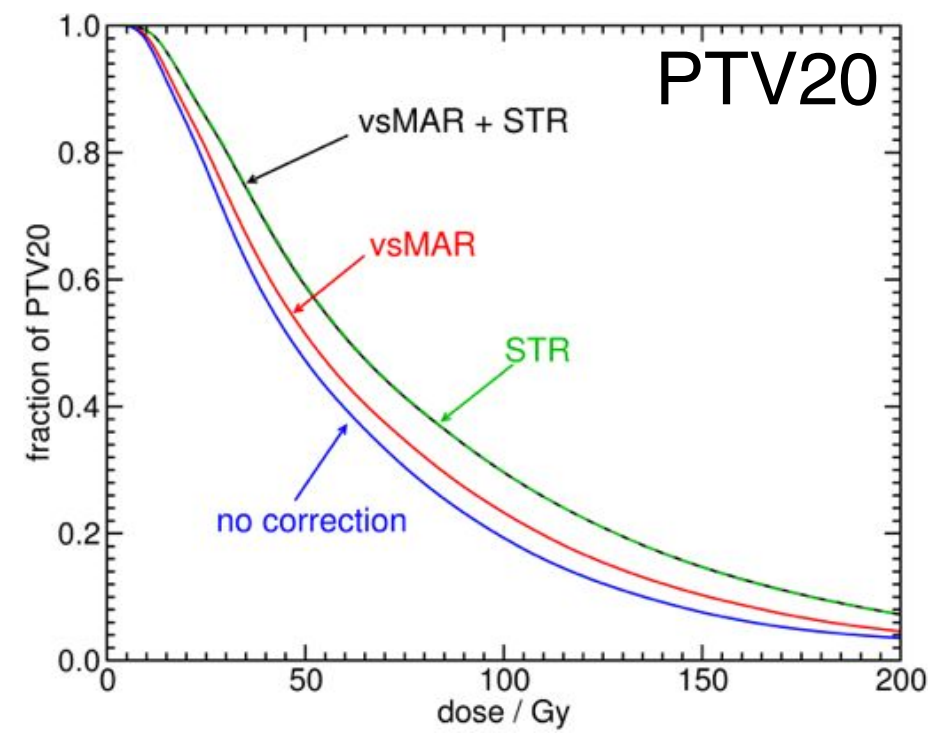

(b)

Figure 4.7: Dose volume histograms for patient 1 with various correction schemes in (a) PTV05 and (b) PTV20. Phantoms with vsMAR + STR correction, vsMAR correction, STR correction and no correction are shown. The simple tissue assignment scheme is used.

produces results that are close to the simple scheme while the detailed scheme differs more significantly. No clear trend in dose metrics or DVHs is seen; agreement or lack thereof tends to vary significantly between patients and dose metrics considered. 
Table 4.4: $\mathrm{V}_{100}$ and $\mathrm{D}_{90}$ for PTV05 and $\mathrm{D}_{90}$ for PTV20 for lung brachytherapy patients using various tissue assignment schemes. For moderate and detailed, the percent differences with simple are shown. All phantoms have vsMAR plus STR correction.

\begin{tabular}{cccc}
\hline \hline Patient & simple & moderate & detailed \\
\hline & $\mathrm{V}_{100}$ (volume fraction) for PTV05 \\
\cline { 2 - 4 } 1 & 0.634 & $0.651(2.7 \%)$ & $0.613(-3.4 \%)$ \\
2 & 0.798 & $0.798(0.0 \%)$ & $0.790(-1.0 \%)$ \\
3 & 0.736 & $0.738(0.3 \%)$ & $0.722(-1.9 \%)$ \\
4 & 0.783 & $0.785(0.3 \%)$ & $0.774(-1.1 \%)$ \\
5 & 0.941 & $0.941(0.0 \%)$ & $0.935(-0.6 \%)$ \\
6 & 0.921 & $0.926(0.5 \%)$ & $0.896(-2.8 \%)$ \\
\cline { 2 - 4 } 1 & \multicolumn{4}{c}{$\mathrm{D}_{90}(\mathrm{~Gy})$ for PTV05 } \\
\cline { 2 - 4 } 2 & 59.2 & $60.0(1.4 \%)$ & $56.7(-4.2 \%)$ \\
2 & 64.4 & $65.5(1.6 \%)$ & $62.6(-2.8 \%)$ \\
3 & 41.0 & $42.4(3.5 \%)$ & $39.6(-3.4 \%)$ \\
4 & 50.7 & $52.9(4.3 \%)$ & $50.8(0.2 \%)$ \\
5 & 130.2 & $131.5(1.0 \%)$ & $122.2(-6.2 \%)$ \\
6 & 107.3 & $109.1(1.6 \%)$ & $98.8(-8.0 \%)$ \\
\cline { 2 - 4 } 1 & \multicolumn{4}{c}{$\mathrm{D}_{90}(\mathrm{~Gy})$ for PTV20 } \\
\cline { 2 - 4 } 2 & 20.7 & $21.3(3.1 \%)$ & $21.0(1.5 \%)$ \\
3 & 16.1 & $28.4(1.1 \%)$ & $28.2(0.4 \%)$ \\
4 & 17.9 & $17.0(4.3 \%)$ & $16.5(1.2 \%)$ \\
5 & 31.3 & $31.8(1.4 \%)$ & $31.2(-0.3 \%)$ \\
6 & 22.9 & $24.1(5.2 \%)$ & $23.3(1.5 \%)$ \\
\hline \hline
\end{tabular}

Figure 4.9 shows a dose profile in patient 5 for the simple, moderate, and detailed schemes as well as a TG43-sim calculation. Figure 4.10 shows the profile line superimposed on the vsMAR+STR corrected phantom. Regions where the dose in all three tissue assignment schemes dips down represent voxels that are assigned to adipose tissue whereas regions where only the dose for the detailed scheme dips represents voxels that are assigned to marrow in the detailed scheme and muscle in the other two schemes.

Phantoms created with a CT number to mass density calibration shifted by \pm 50 HU showed no difference in dose metrics or dose volume histograms. 


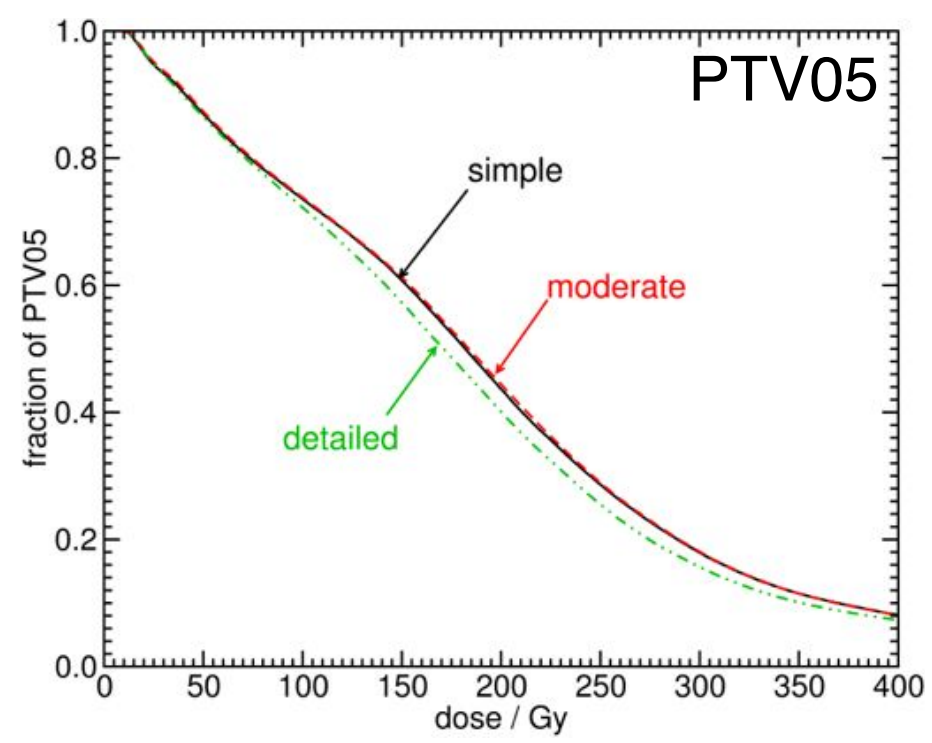

(a)

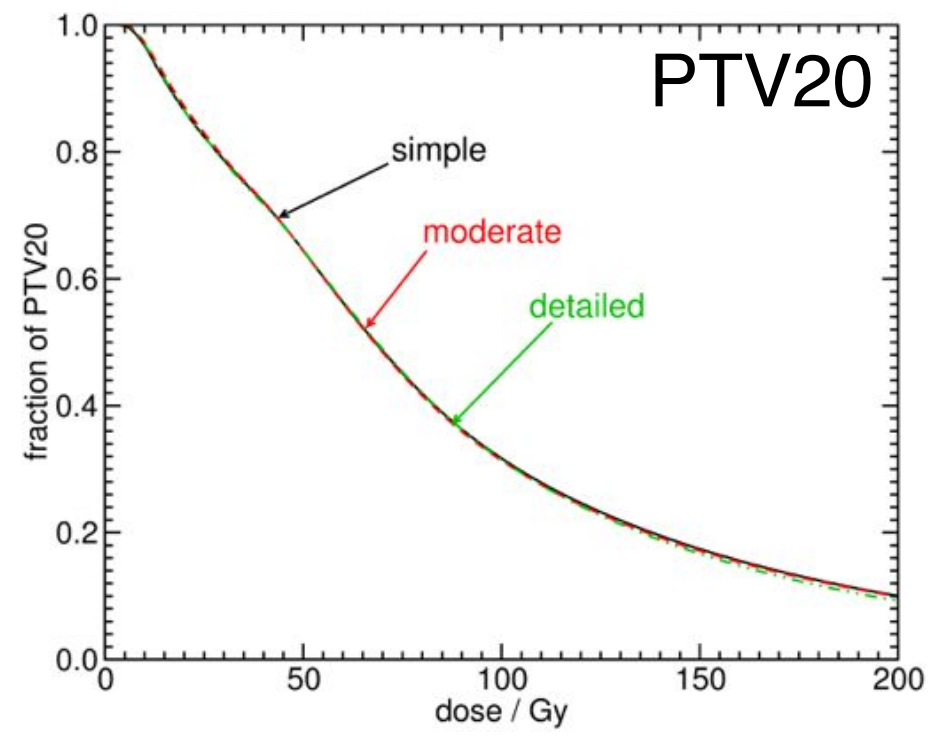

(b)

Figure 4.8: Dose volume histograms for patient 3 with various tissue assignment schemes in (a) PTV05 and (b) PTV20. The simple, moderate, detailed tissue assignment schemes are applied to a vsMAR + STR corrected phantom.

\subsubsection{Comparison with TG-43}

Table 4.5 reports $\mathrm{V}_{100}$ and $\mathrm{D}_{90}$ for PTV05 as well as $\mathrm{D}_{90}$ for PTV20 for all six patients from TG43-sim calculations and from calculations using vsMAR+STR cor- 


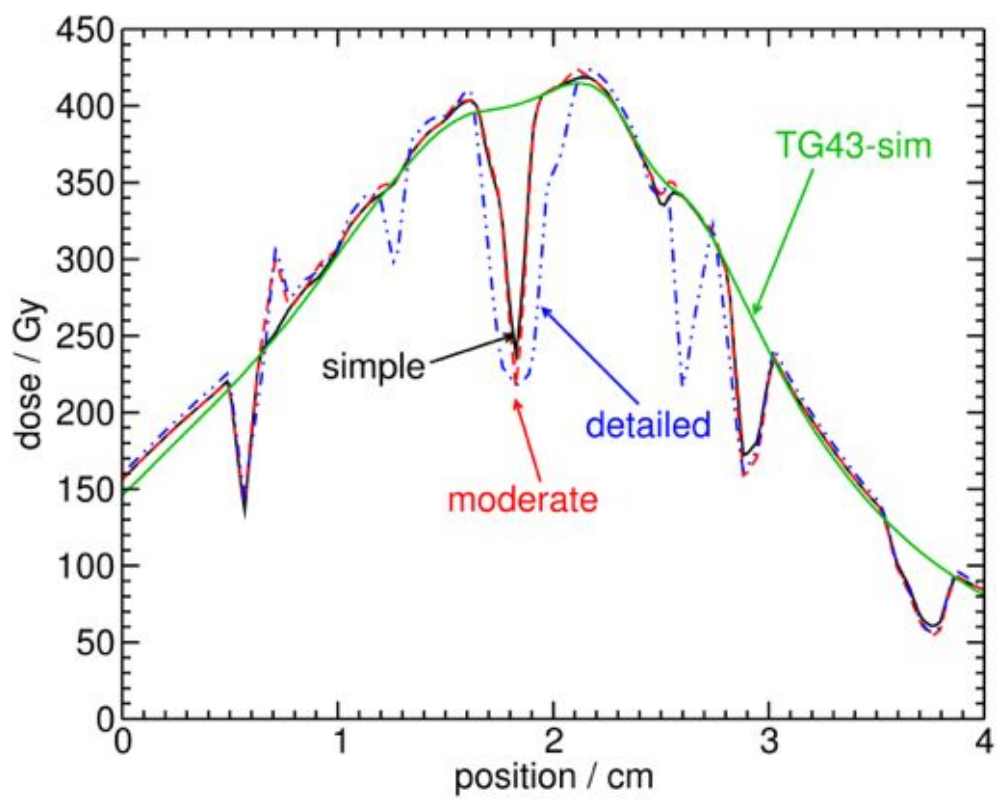

Figure 4.9: Dose profile for patient 5 showing doses in phantoms with vsMAR + STR correction with the simple, moderate, and detailed tissue assignment schemes as well as the TG43-sim dose. The horizontal axis gives the position along the line shown in figure 4.10 .

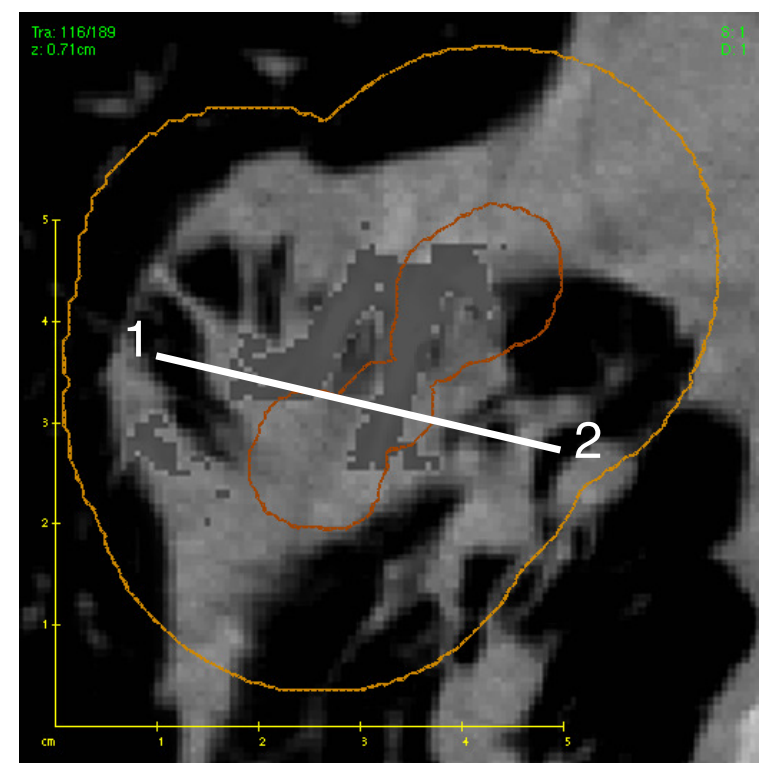

Figure 4.10: CT image for patient 5 showing the line (going from 1 to 2) used for the dose profile in figure 4.9. The outlines for PTV05 and PTV20 are also shown. 
rected phantoms with the simple tissue assignment scheme for both energy deposition in the local tissue in each voxel $\left(\mathrm{D}_{m, m}\right)$ and in water voxels $\left(\mathrm{D}_{w, m}\right)$. In PTV05, agreement in dose metrics from TG43-sim and patient derived phantoms varies considerably between patients; however, a trend of greater disagreement between dose metrics from TG43-sim and patient derived phantoms emerges when considering the PTV20 volumes. This can be seen in figure 4.11 which shows example dose volume histograms for these calculations in PTV05 and PTV20 and this is a result of the relative proportion of lung tissue voxels compared to muscle tissue voxels in the volumes. In PTV05 volumes, the majority of voxels are assigned to muscle whereas in PTV20 volumes, a significant proportion of voxels are less dense and are assigned to lung. In PTV20 volumes, the differences in dose volume histograms and the differences in $\mathrm{D}_{90}$ between TG43-sim and patient derived phantoms generally decreases slightly when dose is scored in water voxels $\left(\mathrm{D}_{w, m}\right)$ versus tissue voxels $\left(\mathrm{D}_{m, m}\right)$.

Figure 4.12 provides example dose profiles (in patient 4 ) of TG43-sim, $\mathrm{D}_{m, m}$, and $\mathrm{D}_{w, m}$ along a line in PTV05 near seeds (Fig. 4.12a) and near the edge of PTV20 in a region composed mainly of lung tissue (Fig. 4.12b). Near the seeds (Fig. 4.12a), the photon energy fluence is relatively similar in water and patient models since patient derived phantom voxels are mostly assigned to muscle and photons have traveled relatively short distances from seeds; thus the effects of differences in density and mass attenuation coefficients are small. However, within PTV05 significant differences between $D_{m, m}$ and $D_{w, m}$ are observed in voxels where the mass energy absorption coefficient differs considerably from that for water. In some regions, $\mathrm{D}_{m, m}$ is closer to TG43-sim than $\mathrm{D}_{w, m}$ indicating that differences in mass energy absorption coefficients are compensating for differences in fluence. Further from the seeds (Fig. 4.12b), differences in photon energy fluence in water and patient models are more substantial, resulting in a different profile shape for TG43-sim compared to $\mathrm{D}_{w, m}$ and $\mathrm{D}_{m, m}$. The 
Table 4.5: $\mathrm{V}_{100}$ and $\mathrm{D}_{90}$ for PTV05 and $\mathrm{D}_{90}$ for PTV20 from calculations of TG43sim and for phantoms with vsMAR plus STR correction and the simple tissue assignment scheme with dose scored in the local medium of each voxel $\left(\mathrm{D}_{m, m}\right)$ and dose scored in water voxels $\left(\mathrm{D}_{w, m}\right)$. The percent difference of TG43-sim from $\mathrm{D}_{m, m}$ and $\mathrm{D}_{w, m}$ calculations are also shown.

\begin{tabular}{|c|c|c|c|c|c|}
\hline Patient & $\overline{\overline{\mathrm{D}_{m, m}}}$ & $\overline{\mathrm{D}_{w, m}}$ & TG43-sim & \% diff. from $\mathrm{D}_{m, m}$ & $\%$ diff. from $\mathrm{D}_{w, m}$ \\
\hline & \multicolumn{5}{|c|}{$\mathrm{V}_{100}$ (volume fraction) for PTV05 } \\
\hline 1 & 0.634 & 0.643 & 0.653 & $2.9 \%$ & $1.6 \%$ \\
\hline 2 & 0.798 & 0.796 & 0.788 & $-1.3 \%$ & $-1.0 \%$ \\
\hline 3 & 0.736 & 0.730 & 0.736 & $0.0 \%$ & $0.9 \%$ \\
\hline 4 & 0.783 & 0.761 & 0.779 & $-0.5 \%$ & $2.4 \%$ \\
\hline 5 & 0.941 & 0.937 & 0.950 & $1.0 \%$ & $1.3 \%$ \\
\hline \multirow[t]{2}{*}{6} & 0.921 & 0.925 & 0.927 & $0.6 \%$ & $0.2 \%$ \\
\hline & \multicolumn{5}{|c|}{$\mathrm{D}_{90}(\mathrm{~Gy})$ for PTV05 } \\
\hline 1 & 59.2 & 61.1 & 62.1 & $4.9 \%$ & $1.6 \%$ \\
\hline 2 & 64.4 & 62.9 & 58.4 & $-9.3 \%$ & $-7.2 \%$ \\
\hline 3 & 41.0 & 39.4 & 40.3 & $-1.7 \%$ & $2.2 \%$ \\
\hline 4 & 50.7 & 47.9 & 59.2 & $16.8 \%$ & $23.5 \%$ \\
\hline 5 & 130.2 & 127.8 & 138.7 & $6.5 \%$ & $8.5 \%$ \\
\hline \multirow[t]{2}{*}{6} & 107.3 & 107.8 & 108.2 & $0.8 \%$ & $0.4 \%$ \\
\hline & \multicolumn{5}{|c|}{$\mathrm{D}_{90}(\mathrm{~Gy})$ for PTV20 } \\
\hline 1 & 20.7 & 19.8 & 16.3 & $-21.0 \%$ & $-17.8 \%$ \\
\hline 2 & 28.1 & 26.6 & 18.1 & $-35.6 \%$ & $-32.0 \%$ \\
\hline 3 & 16.3 & 16.1 & 13.4 & $-17.8 \%$ & $-16.7 \%$ \\
\hline 4 & 17.9 & 16.8 & 14.6 & $-18.7 \%$ & $-13.2 \%$ \\
\hline 5 & 31.3 & 31.0 & 29.0 & $-7.5 \%$ & $-6.5 \%$ \\
\hline 6 & 22.9 & 21.7 & 24.9 & $8.5 \%$ & $14.9 \%$ \\
\hline
\end{tabular}

differences in $\mathrm{D}_{m, m}$ and $\mathrm{D}_{w, m}$ in Fig. 4.12 $\mathrm{p}$ are due to the different mass energy absorption coefficients for lung and water.

\subsection{Discussion}

Four computational phantoms based on patient CT data were constructed using different methods of metallic artifact correction (none, vsMAR, STR, vsMAR+STR). The considerable dose differences between the uncorrected and corrected phantoms underline the importance of correcting CT data. The much lower doses for the uncor- 


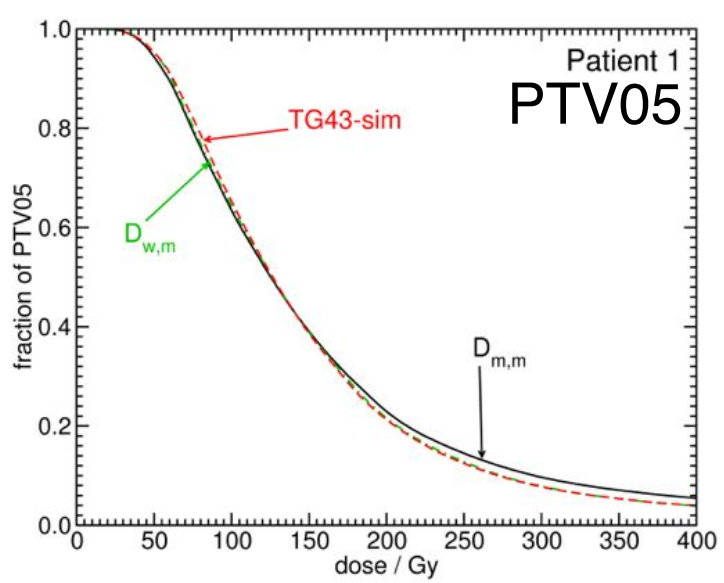

(a)

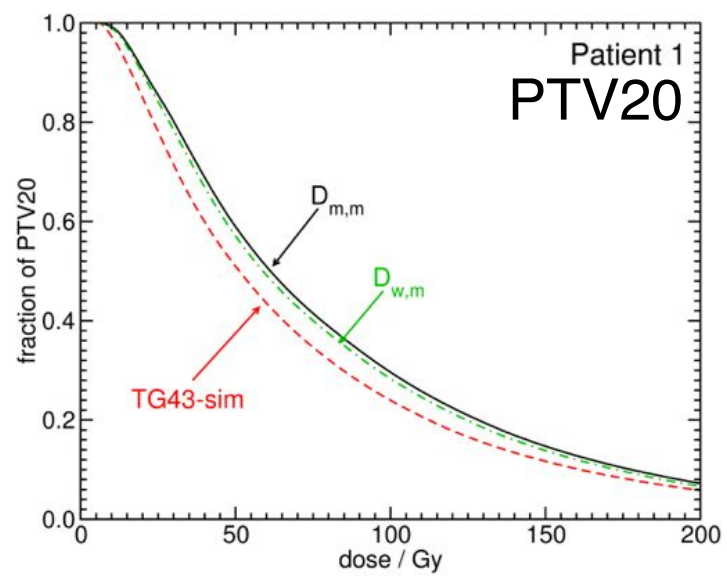

(c)

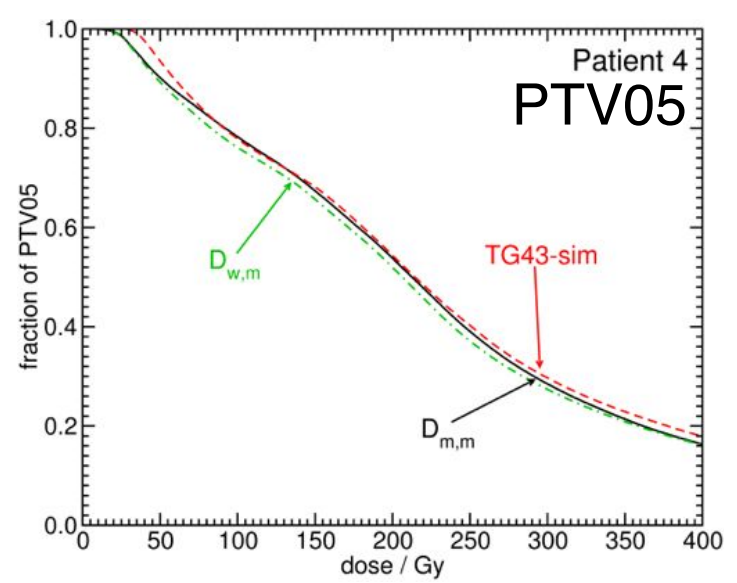

(b)

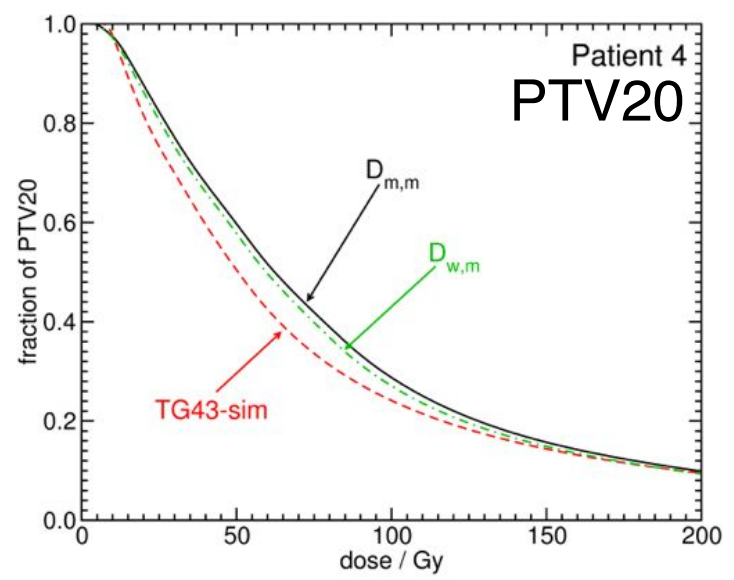

(d)

Figure 4.11: Dose volume histograms comparing TG43-sim and calculations performed with phantoms obtained using vsMAR + STR correction and the simple tissue assignment scheme with dose scored in the local medium of each voxel $\left(\mathrm{D}_{m, m}\right)$ and dose scored in water voxels $\left(\mathrm{D}_{w, m}\right)$ for $(\mathrm{a})$ patient 1 - PTV05, (b) patient 4 - PTV05, (c) patient 1 - PTV20, (d) patient 4 - PTV20.

rected and vsMAR corrected phantoms (up to $40 \%$ for $\mathrm{D}_{90}$ of uncorrected compared to vsMAR+STR) suggests that simply ensuring that emitted photon fluence is realistically simulated (ie. emitted photons pass through realistic tissues) is the most important step in ameliorating the effects of metallic artifacts. The large differences for phantoms with residual seed artifacts arises primarily from seeds being modelled in bone. 


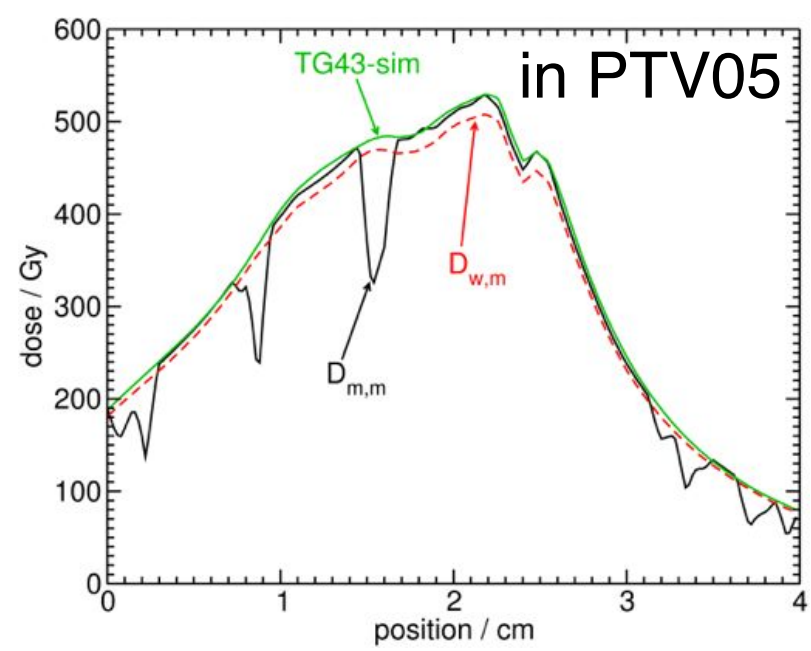

(a)

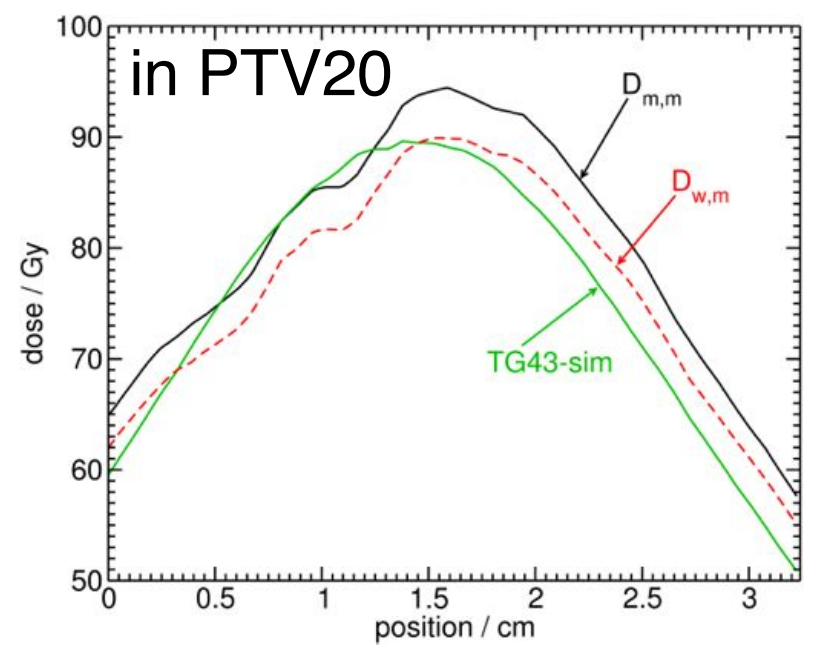

(b)

Figure 4.12: Dose profiles for patient 5 (a) in PTV05 passing near seeds and (b) at the edge of PTV20 passing through mainly lung tissue. Doses are shown from TG43-sim and from calculations performed with phantoms obtained using vsMAR + STR correction and the simple tissue assignment scheme with dose scored in the local medium of each voxel $\left(\mathrm{D}_{m, m}\right)$ and dose scored in water voxels $\left(\mathrm{D}_{w, m}\right)$.

The close agreement between dose volume histograms and dose metrics for the vsMAR+STR and STR only corrected phantoms suggests that the STR method of replacing highly attenuating voxels, though relatively crude, may be clinically sufficient, at least for early adopters of MBDCAs who may want to take advantage 
of the lower computational demand of such a method. However, if local doses in the region of metallic artifacts are desired then more rigorous methods are likely necessary.

The use of raw sinogram data for metallic artifact correction would allow projections passing through metallic objects to be replaced by interpolated values of unaffected projections. This may alleviate the need for supplementary correction. However, the accurate segmentation of seeds in CT images and the resulting delineation of projections in sinograms passing through these seeds still generally poses a challenge in brachytherapy. For example, if one fails to segment every seed completely, then metal influenced projections will be used to interpolate deleted portions of the sinogram. If one uses a wide margin or low CT number to segment seeds, then too many projections may be removed for interpolation to accurately capture the tissue heterogeneity and in extreme cases new artifacts could arise. While recent work ${ }^{[50}$ has shown promising results in prostate treatments, the heterogeneous nature of the implant sites and the possibility of seeds clustering in lung treatments may make accurate application of projection completion-based methods difficult. As such, further study using raw sinograms and investigating other artifact reduction methods ${ }^{52}$ is warranted.

Limited knowledge of the tissues found in the vicinity of the seeds makes voxel tissue assignment in this region a challenge. Although CT numbers for voxels in these regions correspond to adipose or muscle tissues (using the different tissue assignment schemes), it is more probable that these voxels represent fibrotic or scar tissue. However, due to the absence of material composition data for lung fibrotic tissue in the literature and the $\mathrm{CT}$ number range that might represent such tissue, the present work employs the same tissue assignments in the vicinity of the seeds as elsewhere in the phantoms. One might assign only lung tissue with mass density determined 
by voxel CT numbers in the vicinity of the seeds but there is no evidence that the composition of fibrotic tissue is closer to that of healthy lung than to muscle or other tissues. Fibrotic tissues or mixtures of lung and fibrotic tissues might cover a wide range of CT numbers. Regardless, since there is a degeneracy of tissue parameters for a given CT number, and the elemental composition of fibrotic tissue is unknown, the assignment of any tissue is still somewhat arbitrary and represents an uncertainty. The sensitivity of dose distributions on the modelling of fibrotic tissue is an issue that is approached in chapter 5 .

The effect of different tissue assignment schemes on dose varies from patient to patient. The varied nature of the differences that can arise emphasizes the need for a rigorous and accurate method of assigning material compositions. The use of dual energy CT ${ }^{108}$ or other novel methods may be required to determine voxel parameters. Additionally, the uncertainty of the elemental composition of tissues themselves is unclear and more study of tissue composition is required.

This uncertainty in tissue assignment means that absolute comparisons with TG43 doses in the immediate vicinity of the seeds (PTV05) should be undertaken with caution. However, it is possible to conclude that in volumes that contain a significant amount of healthy lung tissue, TG-43 calculations are likely to underestimate the dose by as much as 35\%. Compared to water, lung tissue has a higher mass energy absorption coefficient (approximately $4.7 \%$ difference for $28 \mathrm{keV}$ photons), a lower density, and a relatively similar mass attenuation coefficient (approximately $1.6 \%$ difference for $28 \mathrm{keV}$ photons); lung voxels will attenuate photon fluence less while simultaneously absorbing more dose than water. Thus, the more healthy lung a volume contains, the more TG-43 will underestimate dose as illustrated in table 4.5 and figures 4.11 and 4.12. For the patient data sets considered, scoring energy deposition in water voxels $\left(\mathrm{D}_{w, m}\right)$ rather than in the local tissue $\left(\mathrm{D}_{m, m}\right)$ does not gen- 
erally improve agreement with TG-43 calculated doses; only a small change in percent difference of $\mathrm{D}_{90}$ between TG43-sim and model-based doses is seen in PTV20. This implies that for volumes containing greater proportions of lung tissue, discrepancies between TG-43 and model-based doses are mainly due to differences in calculated photon energy fluence.

Beyond the issues already discussed, there remain a number of unanswered questions that are relevant to using MBDCAs for lung brachytherapy dosimetry. The most immediate are related to the accuracy of using CT data from a snapshot in time to calculate the dose that is absorbed by the patient over weeks. Seed positions may change with time as air kerma strength diminishes, affecting doses delivered. The effects of breathing motion on patient dosimetry is probably much lower than for external beam treatments; this has been studied for ${ }^{192} \mathrm{Ir}$ bile duct implants ${ }^{109}$ but not for ${ }^{125} \mathrm{I}$ lung implants. Additionally, the tissue composition in the patient is likely to change as fibrotic and scar tissue develops and swelling subsides following surgery. In the future, MBDCAs will be instrumental in assessing the impact of these effects on doses delivered.

\subsection{Conclusions}

Full Monte Carlo calculations were performed for six ${ }^{125} \mathrm{I}$ lung brachytherapy patients treated at the Mayo Clinic Rochester using BrachyDose. Different metallic artifact and tissue assignment schemes were considered and their effects on dose distributions were investigated.

Large differences between phantoms that receive metallic artifact correction and no correction underline the necessity of mitigation of the effects of metallic artifacts for MBDCAs for lung brachytherapy. The agreement in DVHs and dose metrics be- 
tween phantoms receiving vsMAR+STR and STR corrections suggest that simpler correction methods may be clinically sufficient providing they correct for voxels that would otherwise cause unrealistically high attenuation of emitted photon fluence. The challenges posed by seed artifacts found in these patients motivates further investigation of metallic artifact reduction schemes for lung brachytherapy in chapter 5 .

The three different tissue assignment schemes used resulted in disagreement in dose metrics that varied unpredictably for each patient. This suggests that a rigorous and accurate method of determining voxel parameters and tissue assignment is necessary. However, results suggest that patient dose is insensitive to small errors in assigning mass density assuming elemental composition remains constant. A source of uncertainty is the composition of the tissue in the vicinity of the seeds which may be lung fibrotic tissue (see chapter 5). Rigorous implementation of MBDCAs may require knowledge of the elemental composition of this tissue.

Despite the uncertainties introduced with MBDCAs, significant differences in DVHs and dose metrics between MBDCAs and TG-43 are likely. Generally, TG-43 calculations underestimate doses in volumes containing significant amounts of healthy lung tissue. 


\section{Chapter 5}

\section{Metallic artifact mitigation and organ-constrained tissue assignment for lung brachytherapy}

As a continuation of the previous chapter which identified the salient modelling issues, this chapter presents an investigation of improved techniques for the accurate calculation of permanent implant lung brachytherapy dose distributions. Specifically, this chapter seeks methods to improve the accuracy of CT-derived computational phantoms by further examining the issue of metallic artifact reduction as well as investigating the practice of constraining tissue assignments within organ contours.

The investigation of metallic artifact reduction techniques is continued by comparing three techniques: the simple threshold replacement technique of chapter 4, a technique involving the filtered back projection of a modified virtual sinogram (an improvement on the vsMAR technique), and a 3D adaptive median filter technique $\sqrt{52}$.

Before accurately assigning discrete tissues from CT numbers, two challenges must be addressed: a number of human tissues are poorly distinguished by CT number with multiple tissues of differing atomic composition corresponding to the same CT number ${ }^{44}$ and voxel CT numbers may correspond to tissues that are anatomically erroneous, particularly in heterogeneous regions such as the lung, due to noise, scatter, 
and voxel volume averaging in CT imaging. Additionally, the presence of fibrotic tissue of unknown atomic composition in lung brachytherapy patients is a source of unresolved uncertainty.

The report of Task Group 186 on model-based dose calculation methods in brachytherapy beyond the TG-43 formalism ${ }^{29}$ recommends that the assignment of tissues be guided by organ contours. Organ-constrained tissue assignment has been shown to affect dose distributions in external beam radiotherapy where dose is less sensitive to atomic composition 110 . By applying separate tissue assignment schemes within organ contours, the mis-assignment of tissues may be reduced and the effect of incomplete artifact correction may be minimized. While organ-constrained tissue assignment schemes may resolve some problems in the assignment of elemental compositions to voxels in computational phantoms, it does not address the incorrect assignment of voxel density due to metallic artifacts. The TG-186 report recommends that if metallic artifacts are present, the density of the tissue in question be over-ridden.

This chapter uses Monte Carlo simulations and data from the lung brachytherapy patients of chapter 4 to investigate metallic artifact mitigation and tissue assignment by calculating dose distributions for a variety of computational phantom models. Computational phantoms are generated from uncorrected CT images as well as CT images on which different metallic artifact reduction techniques have been applied. Tissue assignment schemes are applied both globally or constrained within lung contours. Voxel densities are assigned using a CT number to density calibration or using the nominal density of the voxel tissue. Phantoms with muscle tissue assigned within lung contours are used to investigate the magnitude of dose differences that might arise due to the presence of fibrotic tissue (composition unknown). 


\subsection{Methods}

\subsubsection{Metallic artifact reduction}

The patient CT images of chapter 4 are treated with three metallic artifact reduction techniques. The first method is the "simple threshold replacement" method presented in chapter 4 (page 48). The second method, a fan beam virtual sinogram method labeled "fan", is an improved version of the method used in the previous chapter: in lieu of the radon transform used previously, a built-in MATLAB (The Mathworks, Natick Mass. USA) fan-beam transform is used to create a virtual sinogram. A threshold of $1500 \mathrm{HU}$ is used to delineate segments of the original image corresponding to metallic seeds. Portions of the virtual sinogram corresponding to projections passing through the seeds are deleted and replaced with cubic spline interpolations of adjacent projections. A filtered back-projection is then performed using an inverse fan beam transform on the resulting virtual sinogram to produce the corrected image. For the fan beam transform, an arc sensor geometry is used with a sensor spacing of 0.1 degrees and a rotation increment of 0.1 degrees. The distance in pixels from the fan-beam vertex to the centre of rotation is determined using the distance from source to detector and pixel sizes defined in the CT image meta-information. While the "fan" technique leaves fewer residual artifacts compared to the vsMAR method of chapter 4, some residual artifacts still remain in the immediate vicinity of the sources and so dose calculations are performed with phantoms generated from CT images that receive the "fan" method alone as well as those that receive "fan" followed by STR to help further eliminate these residual artifacts.

The third technique, labeled "median", is a 3D adaptive median filter based on the work of Basran et al. ${ }^{[52}$ but differs slightly since our aim is to remove both metal 
streak artifacts and ${ }^{125} \mathrm{I}$ seeds because BrachyDose models the seeds separately. To ensure that seeds are removed from CT images, a 30 bin histogram of the frequency of CT numbers in the image is created. Any voxel with a CT number greater than $1500 \mathrm{HU}$ is replaced with the CT number that corresponds to the greatest histogram bin that has at least $10 \%$ of image voxels. Next, a region of interest (ROI) of $5 \times 5 \times 7$ voxels is defined around each voxel and two filters are applied. In the first filter, the median CT number and standard deviation of the ROI is calculated and if the voxel CT number is greater or less than 0.25 times the standard deviation from the median value in the ROI, it is replaced by the adjacent voxel value in one of the two neighbouring slices that is nearest to the median. To reduce noise introduced by the first filter, a second filter is applied where the median value of the 3D ROI is computed and replaces each voxel value. The 3D ROI size is chosen so as to enhance artifact reduction while reducing loss of soft tissue detail and other algorithm parameters are chosen for effectiveness.

Table 5.1 summarizes the labels and descriptions of the metallic artifact reduction techniques.

\subsubsection{Tissue assignment schemes and development of computational phantoms}

Phantoms from three representative patients (patients 1,2 and 5) from chapter 4 are chosen for dose calculations. In each case, CT images are resampled to a maximum of 300 voxels in each direction for computational efficiency before being converted to EGSnrc phantom files (.egsphant). Tissue assignment schemes are created consisting of air, lung, adipose, muscle, and cortical bone. Tissue compositions taken from Woodard and White ${ }^{85}$ are given in table 2.1 (page 19). 
Table 5.1: Summary of metallic artifact reduction techniques

\begin{tabular}{cl}
\hline \hline Label & Description \\
\hline uncorr. & Original, uncorrected CT image \\
STR & Simple threshold replacement: Within a $1 \times 1 \times 1 \mathrm{~cm}^{3}$ cube cen- \\
& tred around each seed position, if a voxel CT number is \\
& greater than or equal to $88 \mathrm{HU}$, it is replaced with a CT \\
& number of - $200 \mathrm{HU}$. \\
fan & Fan beam virtual sinogram: A fan beam transform is used \\
& to create a virtual sinogram. Projections passing through \\
& voxels with CT numbers greater than or equal to $1500 \mathrm{HU}$ are \\
& deleted and replaced with spline interpolations of surrounding \\
& projections. A filtered back projection is performed using an \\
& inverse fan beam transform to recreate the image. \\
median & $\begin{array}{l}\text { Median filter: Each voxel value is compared with a 3D local } \\
\text { median value and replaced with an adjacent voxel value if it } \\
\text { differs by } 0.25 \text { times the standard deviation. A second filter } \\
\text { is used to smoth the image. }\end{array}$ \\
\hline \hline
\end{tabular}

Four tissue assignment schemes are used to generate computational phantoms from uncorrected and corrected CT images and are represented in figure 5.1. The first assignment scheme is labeled "unconstrained" and is assigned globally. The second assignment scheme, labeled "lung, air" is identical to "unconstrained" except inside lung contours where only lung and air are assigned. The third scheme, "lung, air, muscle" is similar to "lung, air" but allows the assignment of muscle tissue to approximately investigate the effect of the fibrotic tissue of unknown composition (as fibrotic tissue may form in the vicinity of the implant following surgery). For "unconstrained", "lung, air", and "lung, air, muscle", the mass density of each voxel is determined by the CT number to mass density calibration in table 4.2. The last scheme, "lung, air (nominal)" has the same tissue assignment as "lung, air" but, throughout the phantom, voxel densities are defined as the nominal density of the local voxel tissue. The tissue assignment schemes of this chapter are based on the "simple" scheme of chapter 4 , the CT number boundaries between tissues represent a 
compromise between those listed in the literature ${ }^{44 \mid 45 / 47}$ and the features distinguished from CT images such as the frequency distribution of CT numbers in the phantoms and the CT numbers found in the pneumothorax of one patientt*

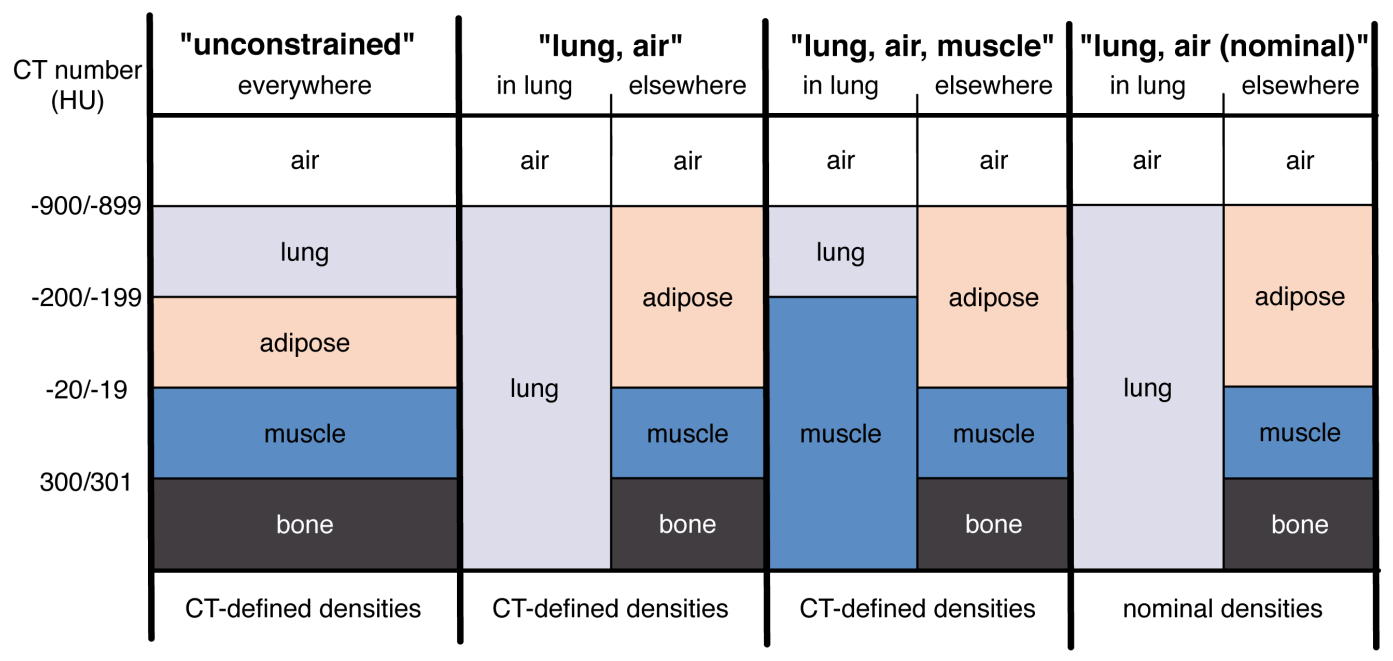

Figure 5.1: The four tissue assignment schemes used: "unconstrained", "lung, air", "lung, air, muscle", and "lung, air (nominal)". CT number boundaries (in HU) are denoted (e.g., in all schemes, CT numbers less than or equal to -900 are assigned to air). The "unconstrained" scheme uses a single tissue assignment scheme that is applied globally to all voxels. The other three schemes assign tissues differently depending on whether they are inside lung contours ("in lung") or outside ("elsewhere") (e.g., for the "lung, air" scheme, a voxel with a CT number of $0 \mathrm{HU}$ will be assigned to lung if it is within the lung contour and assigned to muscle otherwise.) The method of determining voxel mass density (from a CT-mass density calibration or using the nominal mass density of the voxel tissue) is denoted below each scheme.

${ }^{*} \mathrm{CT}$ number boundaries between the tissue assignment schemes of this chapter and those of chapter 4 differ and so dose distributions and metrics are not directly comparable 


\subsection{Results and Discussion}

\subsubsection{Metallic artifact reduction}

Figure 5.2 shows a slice of an uncorrected CT image for patient 2 (section 5.2.2): seeds and streaking artifacts are visible. Figure 5.3 shows the same slice treated using the "STR", "fan", and "median" metallic artifact reduction techniques as well as difference images between treated and uncorrected images. The features observed in figures 5.2 and 5.3 are generalizable to all the images for the six patients.

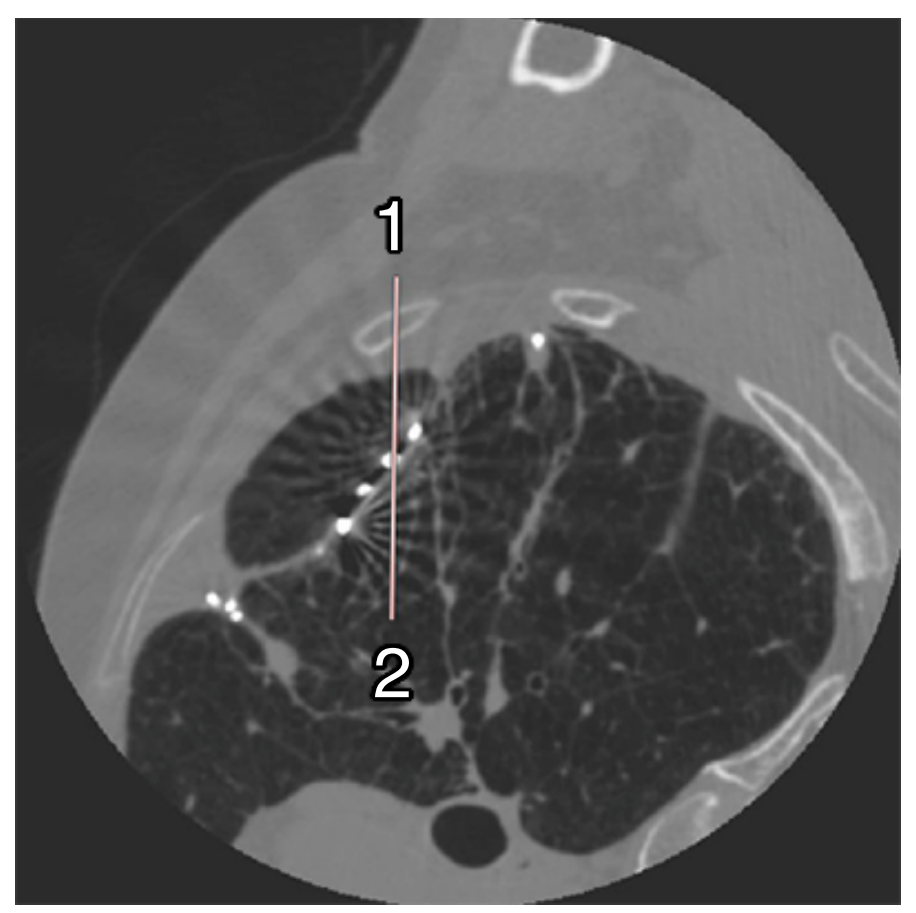

Figure 5.2: A slice of an uncorrected CT image from patient 2. Seeds and metal artifacts are visible. The line (going from 1 to 2 ) denotes the profile used for figures 5.4 and 5.5 .

For "STR" corrected images (figure 5.3 $\mathrm{a}, \mathrm{b}$ ), the high CT numbers in the vicinity of the seeds are reduced but streaking artifacts beyond the region of interest (1 $1 \times 1 \times 1 \mathrm{~cm}^{3}$ cube around each seed) remain unaltered. Suture staples are visible 


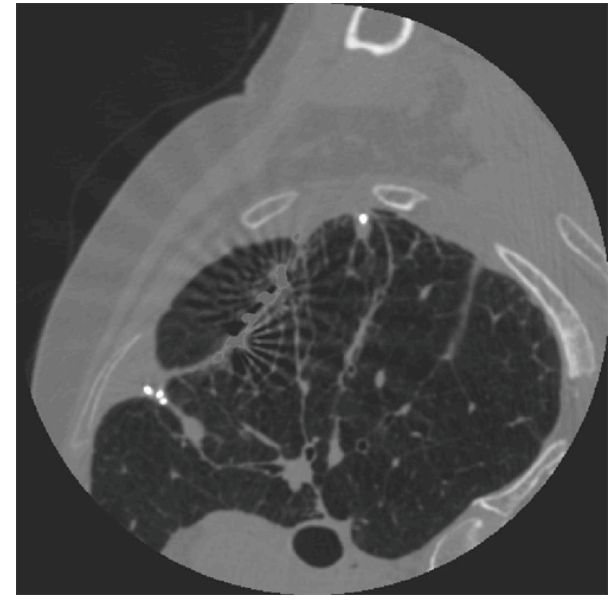

(a)

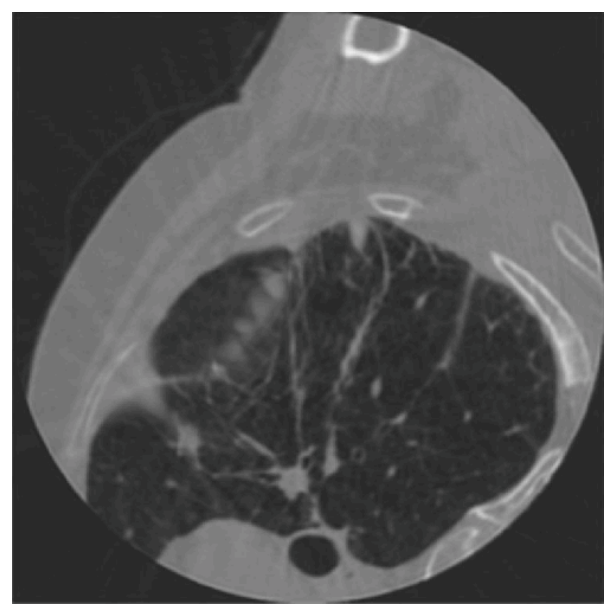

(c)

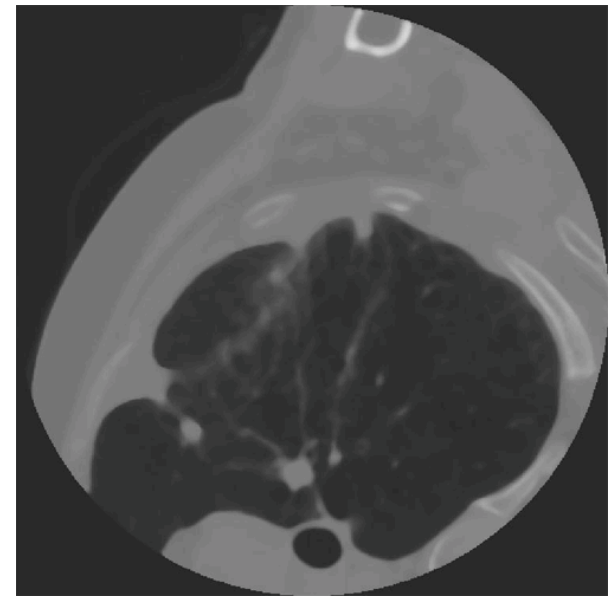

(e)

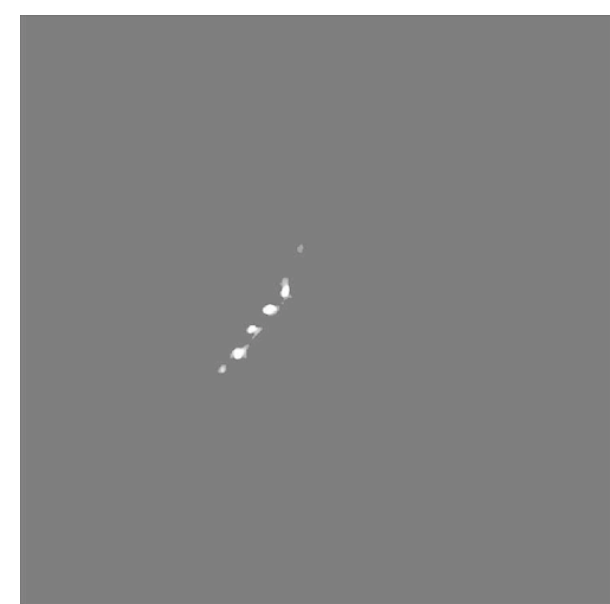

(b)

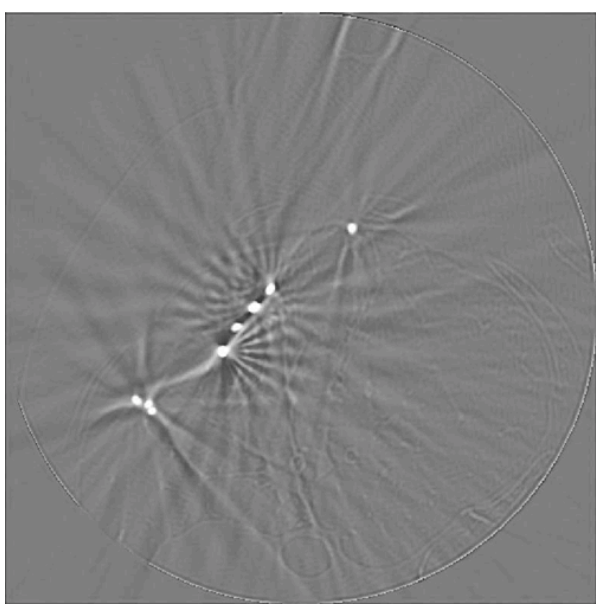

(d)

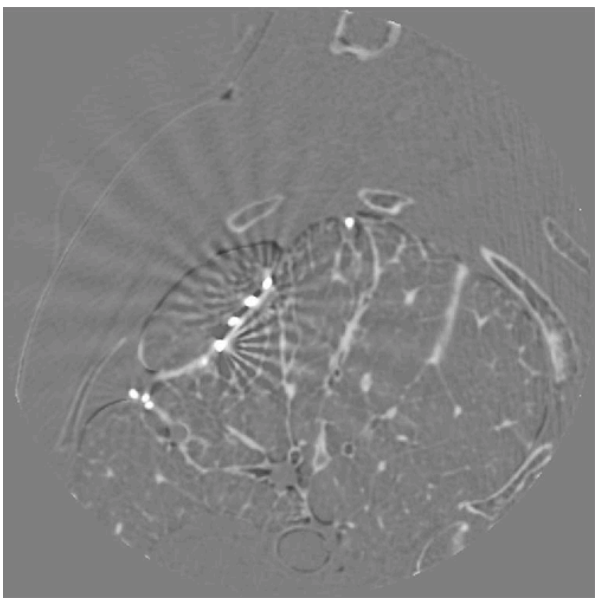

(f)

Figure 5.3: Slices of CT images from patient 2 treated with the (a) "STR", (c) "fan", and (e) "median" metallic artifact reduction techniques. Also shown are difference images between (b) "STR", (d) "fan", (f) "median" treated images and the uncorrected image (figure 5.2 . 
in figure 5.3 (a) and may cause mis-assignment of tissues since metal is not included in tissue assignment schemes. However, the staples are small and are not likely to considerably affect dose. Since the algorithm is only applied in the vicinity of the seeds, elsewhere, soft tissue and bone details from the original image remains intact.

For "fan" corrected images (figure 5.3., d), high CT numbers due to seeds and staples as well as metallic streaking artifacts are reduced; however, some residual artifacts remain and are observed as subtle streaks in figure 5.3c. Soft tissue and bone details are preserved. Since projections that pass near but not through seeds are used for the spline interpolations in the virtual sinograms, interpolations are calculated using data that has itself been affected by streaking artifacts, possibly contributing to the residual artifacts. Metallic artifacts are more severe in the immediate vicinity of the seeds and so one might consider adding a margin to voxels corresponding to seed positions when delineating projections in virtual sinograms that correspond to seeds. This would mean that interpolations over deleted segments of virtual sinograms are performed using data that is less affected by artifacts but over a larger gap of missing data. Selecting the projections in virtual sinograms to delete and replace with interpolations, either by adjusting the CT number threshold that corresponds to seeds or by adding margins around detected seeds, requires finding a balance: if deleted segments are too large then interpolation breaks down and new artifacts are created, however, smaller deletions leave existing artifacts and high CT numbers uncorrected. Access to original sinogram data might make this balance easier to achieve as projections passing near but not through seeds are less likely to be affected by metallic artifacts in original sinograms. However, it is unclear whether any benefit gained from using original sinogram data would justify the greater complexity of adopting such an approach clinically.

For "median" corrected images (figure 5.3 ,f), high CT numbers due to seeds 
and metallic streaking artifacts are reduced with no residual artifacts visible. Suture staples are also removed. However, while the second smoothing filter reduces noise, it also impacts some of the heterogeneities present in the images. This is most apparent in the loss of detail in the lung as well as a reduction of intensity in ribs. The median filter method introduced by Basran et al. ${ }^{52}$ uses a CT number threshold of $2000 \mathrm{HU}$ above which voxels are left unchanged. This is to preserve CT numbers that correspond to bone and seeds. However, our goal is to remove seeds from CT images so that they can be modelled separately by BrachyDose, and so the use of such a threshold is inappropriate. Basran et al. also discuss how the median filter reduces contrast within regions with a large range of CT intensities and suggest modifying the filter to ignore voxels with very low CT numbers and CT numbers corresponding to bone. Unfortunately, since lung brachytherapy implants are found in regions with large ranges of CT number, such an approach would greatly reduce the ability of the median filter to reduce metallic artifacts in the images used in this study.

For each of the images shown in figures 5.2 (page 78) and 5.3 (page 79), figure 5.4a presents the CT number profile along the line shown in figure 5.2, while figure 5.4b displays the tissue that would be assigned along that profile if the "unconstrained" tissue assignment were used for each slice. All three metallic artifact reduction schemes reduce the high CT numbers associated with the seed at approximately $4 \mathrm{~cm}$. Beyond this region, the CT numbers for the "STR" treated image exactly match those for the uncorrected image. As a result, the unmitigated streaking artifacts seen between 4 and $7 \mathrm{~cm}$ result in the erroneous assignment of voxels to air and adipose for these two images. Both "fan" and "median" treated profiles reduce high CT numbers due to seeds and streaking artifacts sufficiently such that lung tissue is properly assigned although the density assigned along the profiles would differ between the two. The two peaks in CT number at approximately $1 \mathrm{~cm}$ corresponding to a rib are not fully 
preserved in the "median" treated profile and consequently these voxels would not be assigned to bone. Additionally, both "fan" and "median" treated images can raise or lower the CT numbers at tissue boundaries, effectively changing the positions at which the boundaries between assigned tissues occur, e.g. at $2 \mathrm{~cm}$ along the profile in figure 5.4 .

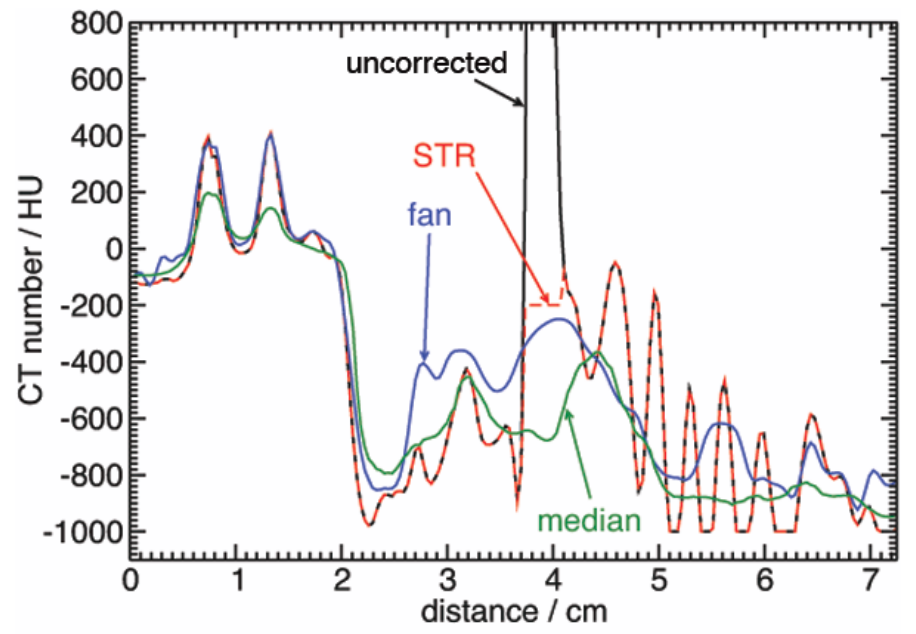

(a)

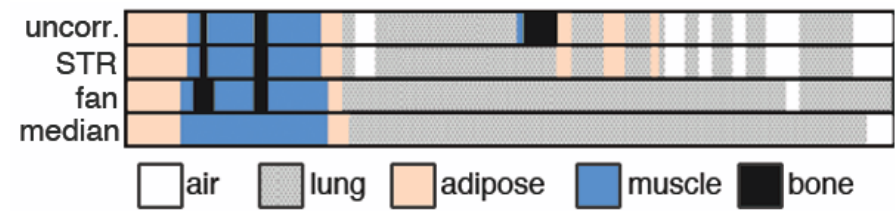

Figure 5.4: (a) CT number profile for patient 2 for uncorrected, STR, fan, and median CT images. The horizontal axis gives the position along the line shown in figure 5.2. (b) Diagram showing the tissue assigned to each voxel in the profile (a) based on the "unconstrained" tissue assignment scheme for each CT image.

The three metallic artifact reduction techniques have varying effects on image quality. The simple threshold replacement leaves image quality unchanged compared with the uncorrected image. The "fan" technique introduces noise in the form of new or residual artifacts and the "median" technique reduces image noise. These differences will affect calculated dose distributions only insofar as they affect the assignment of voxel media and, to a lesser degree, voxel mass density. 


\subsubsection{Tissue assignment schemes and dose}

Tables $5.2-5.4$ provide the minimum dose in the hottest $90 \%$ and the volume $\left(\mathrm{D}_{90}\right)$ and the percent volume that receives at least the prescription dose of $100 \mathrm{~Gy}\left(\mathrm{~V}_{100}\right)$ for PTV05 and PTV20 for patients 1, 2, and 5, respectively. Universally, the dose metrics calculated using uncorrected, unconstrained phantoms are lower than for metallic artifact reduced and/or constrained phantoms due to higher CT numbers in the vicinity of seeds resulting in voxels being assigned to dense bone (which considerably attenuates photons emitted from the seeds). Table 5.5 lists the percentage of voxels that are assigned to bone in the immediate vicinity of seeds (which is defined as any voxel within a $1 \times 1 \times 1 \mathrm{~cm}^{3}$ cube of a seed centre, corresponding to the volumes used for "STR") for a subset of phantom models. Visual inspection of CT images indicates that a very small percentage of voxels in the seed ROIs of patients 1 and 2 are found in the ribs but that no voxels in the seed ROIs of patient 5 are found in anatomical bone. In agreement with the results of chapter 4, the mis-assignment of voxels to bone causes the greatest difference in dose distributions between various models.

For all three patients, dose metrics increase dramatically when metallic artifact correction methods are used. Dose metrics in unconstrained phantoms for patient 1 are approximately equal for phantoms that received "STR" and "fan+STR", but differ in phantoms that received "fan" and "median". For patient 2, "STR", "fan+STR", and "median" treated phantoms have dose metrics that differ from those of "fan" treated phantoms for patient 2. In patient 5, D 90 for PTV05 differs considerably between phantoms derived using all four metallic artifact reduction techniques. However, the considerably lower dose for "fan" and the relatively high percentage of voxels assigned to bone for the "fan" unconstrained phantom for this patient (which should have no bone in the vicinity of the seeds), suggests that the "fan" method did 
not sufficiently reduce erroneously high CT numbers in the vicinity of seeds. Additionally, the lower dose for the "median" phantom and the relatively higher percentage of voxels assigned to bone for this phantom compared to those of patients 1 and 2 , suggest that the "median" technique also did not sufficiently reduce erroneously high CT numbers in the vicinity of seeds.

Comparison of phantoms created with "unconstrained" and "lung, air" tissue assignments illustrates the effect of constraining tissue assignment schemes. Dose metrics for phantoms created with constrained tissue assignment schemes are larger than for those using the unconstrained scheme, partially due to reduced mis-assignment

Table 5.2: $\mathrm{D}_{90}$ and $\mathrm{V}_{100}$ for PTV05 and PTV20 for the various computational phantoms for patient 1 .

\begin{tabular}{|c|c|c|c|c|c|}
\hline \multirow[b]{2}{*}{ MAR } & \multirow[b]{2}{*}{ assign. scheme } & \multicolumn{2}{|c|}{ PTV05 } & \multicolumn{2}{|c|}{ PTV20 } \\
\hline & & $\mathrm{D}_{90}(\mathrm{~Gy})$ & $\mathrm{V}_{100}(\%)$ & $\mathrm{D}_{90}(\mathrm{~Gy})$ & $\mathrm{V}_{100}(\%)$ \\
\hline \multirow{4}{*}{ uncorr. } & unconstrained & 42.1 & 41.9 & 15.3 & 18.6 \\
\hline & lung, air & 63.0 & 64.9 & 19.7 & 28.3 \\
\hline & lung, air, muscle & 62.7 & 64.4 & 19.7 & 28.2 \\
\hline & lung, air (nom.) & 85.5 & 82.4 & 25.9 & 35.5 \\
\hline \multirow{4}{*}{ STR } & unconstrained & 55.4 & 60.3 & 20.0 & 28.9 \\
\hline & lung, air & 66.1 & 68.2 & 20.8 & 30.1 \\
\hline & lung, air, muscle & 65.7 & 67.6 & 20.8 & 30.0 \\
\hline & lung, air (nom.) & 85.9 & 82.5 & 26.6 & 36.2 \\
\hline \multirow{4}{*}{ fan } & unconstrained & 52.9 & 57.2 & 20.3 & 27.9 \\
\hline & lung, air & 65.3 & 67.3 & 20.9 & 29.9 \\
\hline & lung, air, muscle & 65.0 & 66.7 & 21.0 & 29.7 \\
\hline & lung, air (nom.) & 85.9 & 82.5 & 27.1 & 36.1 \\
\hline \multirow{4}{*}{ fan+STR } & unconstrained & 55.0 & 59.4 & 20.5 & 28.9 \\
\hline & lung, air & 65.8 & 67.9 & 20.8 & 30.0 \\
\hline & lung, air, muscle & 65.5 & 67.3 & 20.8 & 29.9 \\
\hline & lung, air (nom.) & 85.7 & 82.3 & 26.7 & 36.1 \\
\hline \multirow{4}{*}{ median } & unconstrained & 57.4 & 61.0 & 21.8 & 28.8 \\
\hline & lung, air & 65.9 & 67.7 & 21.9 & 29.5 \\
\hline & lung, air, muscle & 65.5 & 67.1 & 21.9 & 29.5 \\
\hline & lung, air (nom.) & 87.2 & 83.2 & 28.8 & 36.3 \\
\hline
\end{tabular}


Table 5.3: $\mathrm{D}_{90}$ and $\mathrm{V}_{100}$ for PTV05 and PTV20 for the various computational phantoms for patient 2 .

\begin{tabular}{cccccc}
\hline \hline \multirow{2}{*}{ MAR } & & \multicolumn{2}{c}{ PTV05 } & \multicolumn{2}{c}{ PTV20 } \\
& assign. scheme & $\mathrm{D}_{90}(\mathrm{~Gy})$ & $\mathrm{V}_{100}(\%)$ & $\mathrm{D}_{90}(\mathrm{~Gy})$ & $\mathrm{V}_{100}(\%)$ \\
\hline \multirow{4}{*}{ uncorr. } & unconstrained & 37.9 & 63.6 & 17.3 & 19.0 \\
& lung, air & 59.5 & 78.3 & 25.2 & 30.4 \\
& lung, air, muscle & 59.2 & 78.2 & 25.3 & 30.4 \\
& lung, air (nom.) & 78.3 & 83.9 & 33.2 & 34.8 \\
\multirow{5}{*}{ STR } & unconstrained & 58.8 & 77.7 & 26.2 & 32.0 \\
& lung, air & 63.4 & 79.8 & 26.8 & 32.1 \\
& lung, air, muscle & 63.0 & 79.7 & 26.8 & 32.1 \\
& lung, air (nom.) & 78.4 & 83.9 & 33.2 & 34.9 \\
& unconstrained & 51.6 & 74.8 & 24.4 & 30.8 \\
& lung, air & 61.7 & 79.2 & 26.2 & 31.8 \\
& lung, air, muscle & 61.5 & 79.1 & 26.3 & 31.8 \\
& lung, air (nom.) & 78.3 & 83.9 & 33.0 & 34.7 \\
& & & & & \\
\multirow{5}{*}{ fan+STR } & unconstrained & 58.6 & 77.7 & 26.5 & 32.2 \\
& lung, air & 63.3 & 79.8 & 26.7 & 32.1 \\
& lung, air, muscle & 63.0 & 79.78 & 26.7 & 32.1 \\
& lung, air (nom.) & 78.4 & 83.9 & 33.1 & 34.8 \\
& & & & & \\
median & unconstrained & 58.9 & 78.1 & 27.3 & 32.7 \\
& lung, air & 64.5 & 79.9 & 27.1 & 32.4 \\
& lung, air, muscle & 64.1 & 79.9 & 27.1 & 32.4 \\
& lung, air (nom.) & 78.3 & 84.0 & 32.9 & 34.2 \\
\hline \hline
\end{tabular}


Table 5.4: $\mathrm{D}_{90}$ and $\mathrm{V}_{100}$ for PTV05 and PTV20 for the various computational phantoms for patient 5 .

\begin{tabular}{cccccc}
\hline \hline \multirow{2}{*}{ MAR } & & \multicolumn{2}{c}{ PTV05 } & \multicolumn{2}{c}{ PTV20 } \\
& assign. scheme & $\mathrm{D}_{90}(\mathrm{~Gy})$ & $\mathrm{V}_{100}(\%)$ & $\mathrm{D}_{90}(\mathrm{~Gy})$ & $\mathrm{V}_{100}(\%)$ \\
\hline uncorr. & unconstrained & 68.1 & 77.4 & 18.6 & 18.4 \\
& lung, air & 92.6 & 87.8 & 24.5 & 25.7 \\
& lung, air, muscle & 92.3 & 87.7 & 24.5 & 25.7 \\
& lung, air (nom.) & 102 & 90.6 & 28.4 & 29.5 \\
\multirow{5}{*}{ STR } & unconstrained & 116 & 92.7 & 30.1 & 32.7 \\
& lung, air & 124 & 93.9 & 31.6 & 33.8 \\
& lung, air, muscle & 124 & 93.9 & 31.6 & 33.8 \\
& lung, air (nom.) & 135 & 96.0 & 35.2 & 37.1 \\
& unconstrained & 94.7 & 88.8 & 26.3 & 28.6 \\
& lung, air & 109 & 91.8 & 28.4 & 30.5 \\
& lung, air, muscle & 109 & 91.8 & 28.5 & 30.5 \\
& lung, air (nom.) & 118 & 94.0 & 31.9 & 33.7 \\
& & & & & \\
fanconstrained & 114 & 92.3 & 30.3 & 32.3 \\
& uncong, air & 123 & 93.7 & 31.3 & 33.3 \\
& lung, air, muscle & 122 & 93.7 & 31.3 & 33.3 \\
& lung, air (nom.) & 134 & 95.9 & 35.0 & 36.9 \\
& & & & & \\
& unconstrained & 110 & 91.6 & 30.2 & 31.3 \\
median & lung, air & 123 & 93.7 & 30.4 & 32.0 \\
& lung, air, muscle & 124 & 93.6 & 30.5 & 32.0 \\
& lung, air (nom.) & 139 & 96.3 & 34.6 & 35.8 \\
\hline \hline
\end{tabular}


Table 5.5: Percentage of voxels assigned to bone within the volume defined by the combination of $1 \times 1 \times 1 \mathrm{~cm}^{3}$ cubes around each seed position for patients 1,2 and 5 .

\begin{tabular}{ccccc}
\hline \hline \multirow{2}{*}{ MAR } & assign. scheme & Patient 1 & Patient 2 & Patient 5 \\
\hline \multirow{2}{*}{ uncorr. } & unconstrained & 4.4 & 5.1 & 10 \\
& lung, air & 0.48 & 0.80 & 4.5 \\
\multirow{2}{*}{ STR } & unconstrained & 0 & 0 & 0 \\
& lung, air & 0 & 0 & 0 \\
\multirow{5}{*}{ fan } & unconstrained & 1.2 & 1.8 & 4.1 \\
& lung, air & 0.073 & 0.74 & 1.8 \\
fan+STR & unconstrained & 0 & 0 & 0 \\
& lung, air & 0 & 0 & 0 \\
& unconstrained & 0.041 & 0.073 & 0.88 \\
& lung, air & 0.041 & 0.049 & 0.49 \\
\hline \hline
\end{tabular}

of voxels to bone. This is illustrated in the dramatic increase in $\mathrm{D}_{90}$ for uncorrected phantoms created with "lung, air" versus "unconstrained". However, improvement in the accuracy of dose metrics from constraining tissue assignments within the lung is contingent on seed positions being within the lung contours. For patients 1 and 2, all 40 seeds are found within the lung contours and, consequently, phantoms created from uncorrected images but with constrained assignment ("lung, air") have dose metrics that are in reasonable agreement with phantoms that have been treated with metallic artifact reduction techniques and the same constrained tissue assignment scheme. Conversely, only 27 of the 40 seeds for patient 5 are found within the lung contour and as a result, the dose metrics for the uncorrected "lung, air" phantom are considerably lower than those of the "lung, air" phantoms that received metallic artifact reduction.

Additionally, $\mathrm{D}_{90}$ is greater for phantoms with constrained tissue assignments than those created using the unconstrained scheme even for phantoms where metallic artifact reduction is assumed to have been effective. This implies that the erroneous 
assignment of adipose tissue in phantoms created using unconstrained tissue assignments causes a small but notable decrease in dose metrics since the mass energy absorption coefficient of adipose tissue is approximately $40 \%$ lower than that of lung tissue.

The effects of metallic artifact reduction and constrained tissue assignment presented thus far are demonstrated in figure 5.5 which shows dose profiles along the line shown in figure 5.2 (page 78) for phantoms derived from uncorrected and metallic artifact corrected images using the "unconstrained" (fig. 5.5a) and "lung, air" (fig. 5.5b) tissue assignment schemes. In figure 5.5a, the dose for the phantom that received no artifact mitigation is considerably lower throughout due to the attenuation of photons passing through erroneous bone tissue. The dose profiles for "STR", "fan", and "median" treated phantoms are generally in reasonable agreement with a few exceptions: near $1 \mathrm{~cm}$, the phantom that received the "median" algorithm does not model the rib (see figure $5.4 \mathrm{~b}$ ) resulting in a lower dose in these voxels; between 4 and $6 \mathrm{~cm}$, voxels of air and adipose tissue in the phantom that received "STR" absorb less dose than the lung voxels in the phantoms that received "fan" or "median"; and at approximately $0.6 \mathrm{~cm}$ where more voxels are assigned bone in for the "fan" treated phantom and consequently the dose is higher in those voxels. In figure $5.5 \mathrm{~b}$, voxels beyond $2 \mathrm{~cm}$ from the start of the profile are inside the lung contour and are only assigned to lung or air for each phantom. Consequently, the dose profiles are all in reasonable agreement with only small differences in dose due to differences in voxel densities.

Phantoms created with constrained tissue assignments that allow lung, air, and muscle to be assigned within the lung contour produce dose metrics that are in close agreement with phantoms that have only lung and air within lung contours (tables $5.2,5.4$. While lung and muscle tissues differ in atomic composition, their mass at- 


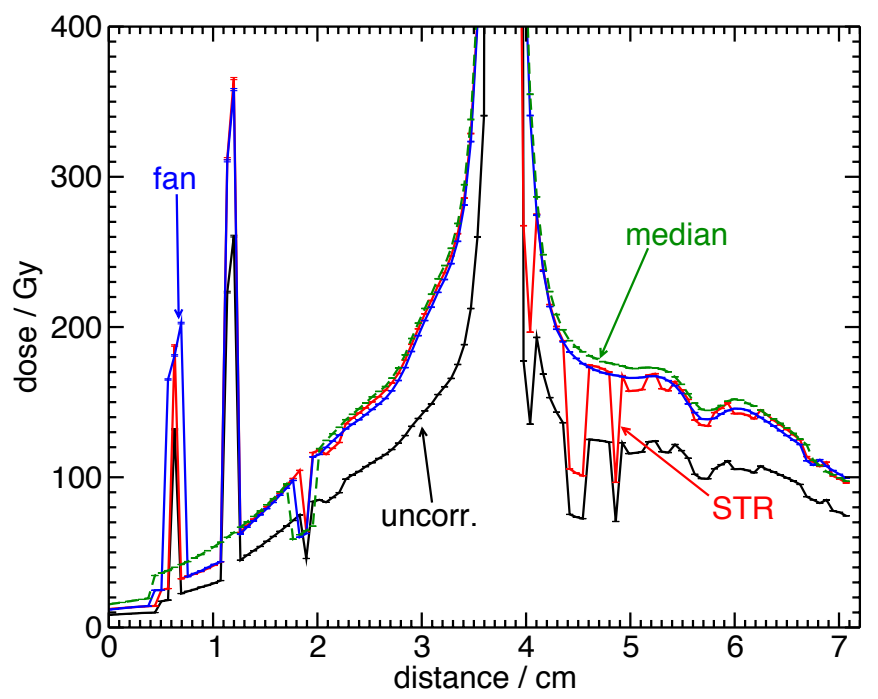

(a)

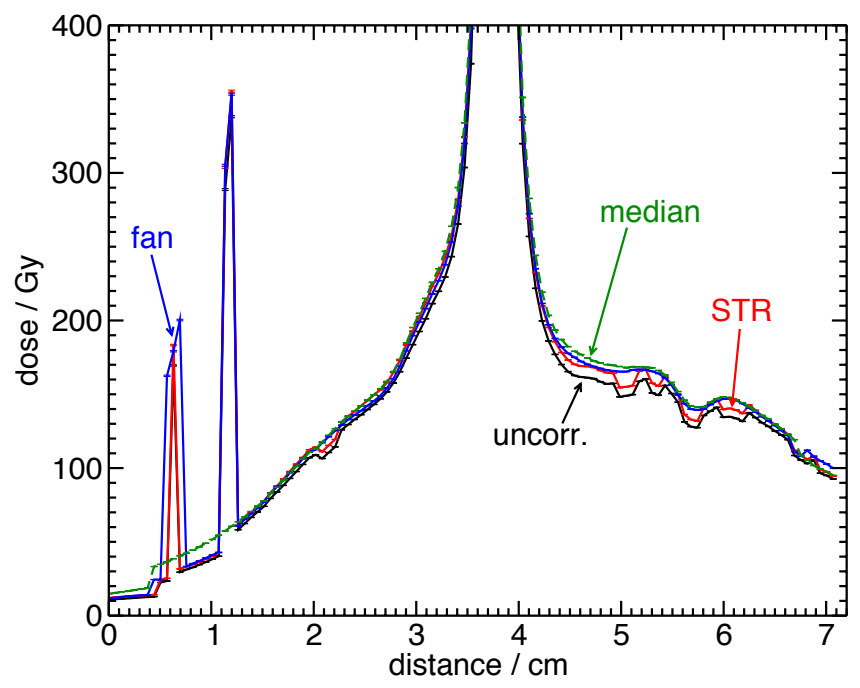

(b)

Figure 5.5: Dose profiles for patient 2 calculated using computational phantoms derived from uncorrected, STR, fan, and median treated images using the (a) "unconstrained" and (b) "lung, air" tissue assignment schemes. The horizontal axes give the position along the line shown in figure 5.2 .

tenuation and absorption coefficients only differ by approximately $0.9 \%$ and $1.5 \%$, respectively, for $28 \mathrm{keV}$ photons ${ }^{111}$. This implies that, so long as the atomic composition of lung fibrotic tissue is relatively similar to that of lung or muscle, it can be omitted in tissue assignment schemes without considerably affecting calculated dose 
distributions.

Dose metrics for phantoms generated using the "lung, air (nominal)" tissue assignment schemes are considerably greater than those for tissue assignments schemes that assigned voxel densities based on CT number. This results from the fact that tissues present in the lung can cover a range of densities and, in the vicinity of the ${ }^{125}$ I implants, may contain higher density fibrotic tissue. As a consequence, phantoms that have voxel densities derived from CT numbers will have higher density voxels in the vicinity of the seeds compared to phantoms where all lung voxels are assigned the nominal density of lung $\left(0.26 \mathrm{~g} / \mathrm{cm}^{3}\right)$. Thus, photon fluence will be attenuated more in these CT-density derived phantoms and doses will be lower than in nominal density phantoms. This is illustrated in figure 5.6 which shows dose and density profiles for phantoms of patient 5 derived from "fan" treated images using the "lung, air" and "lung, air (nominal)" tissue assignment schemes. The lines used for the profiles of figure 5.6 are shown in figure 5.7 which displays composite images of dose distributions and densities for the two phantoms. In figure 5.6, at the start of the profile, both the doses and densities of the CT-defined ("lung, air") and nominal ("lung, air (nominal)") phantoms are approximately equal. As the profile moves farther from the region of the seeds, between approximately 0.25 and $1 \mathrm{~cm}$, the density of the "lung, air" phantom is higher than the "lung, air (nominal)" phantom and the dose along the profile in the "lung, air (nominal)" phantom becomes increasingly greater than that of the "lung, air" phantom. Beyond about $1 \mathrm{~cm}$, the densities for both phantoms are approximately equal and the doses in both phantoms stop diverging. Given the inability of nominal density phantoms to be used to accurately simulate photon fluence, nominal density phantoms are not suitable for overcoming erroneous density assignments caused by uncorrected metallic artifacts in lung brachytherapy patient CT images. 


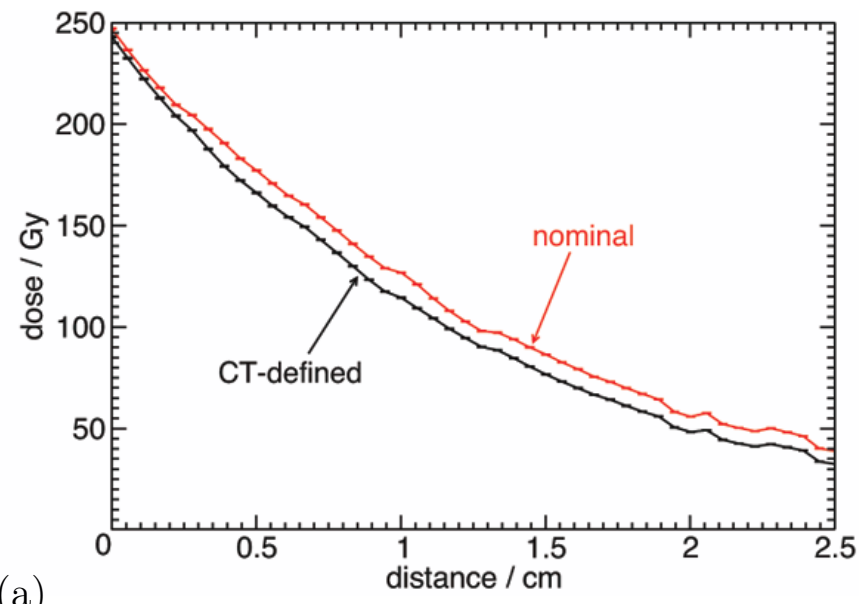

(a)

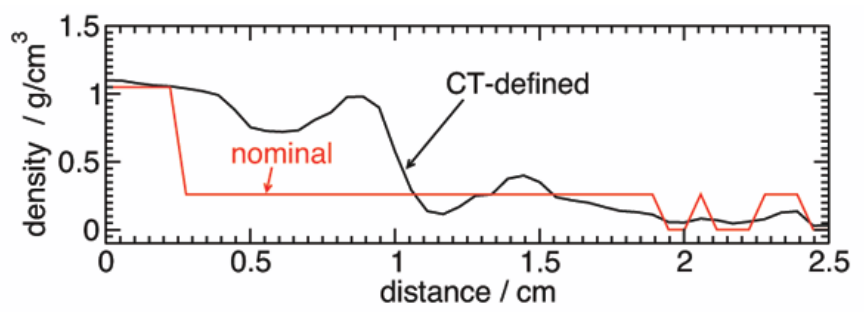

(b)

Figure 5.6: (a) Dose and (b) density profiles for the computational phantoms for patient 5 derived from "fan" treated images using CT-defined ("lung, air") and nominal ("lung, air (nominal)") densities. The horizontal axes give the position from left to right along the lines shown in figure 5.7.

\subsection{Conclusions}

Four metallic artifact reduction methods were applied to CT images for six ${ }^{125} \mathrm{I}$ lung brachytherapy patients treated at the Mayo Clinic Rochester. Simple threshold replacement ("STR") completely removes high CT numbers due to the presence of seeds and metallic streaking artifacts in the immediate vicinity of the seeds. Beyond the vicinity of the seeds, CT numbers are identical to the original image, preserving both soft tissue details and streaking artifacts. The fan beam virtual sinogram method ("fan") reduces high CT numbers due to seeds and metallic streaking artifacts throughout the images while preserving soft tissue detail. However, the "fan" algo- 


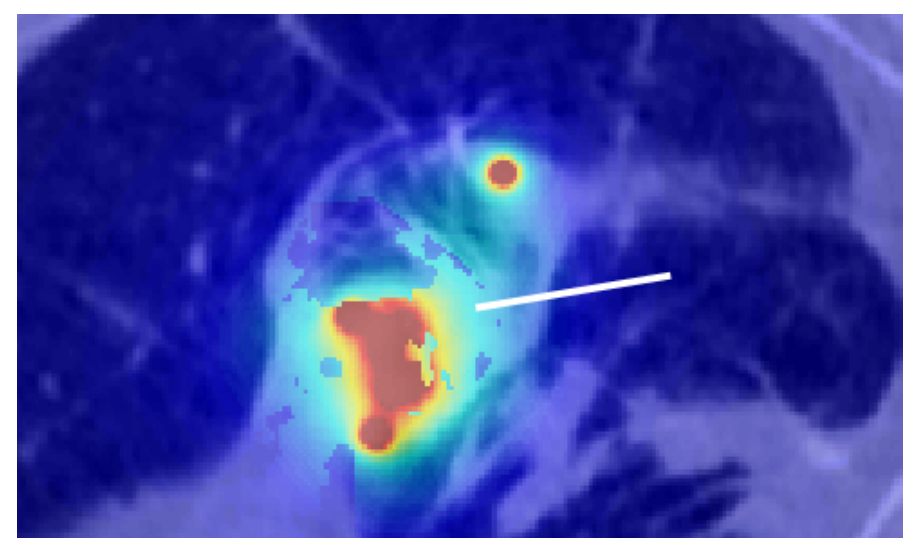

(a)

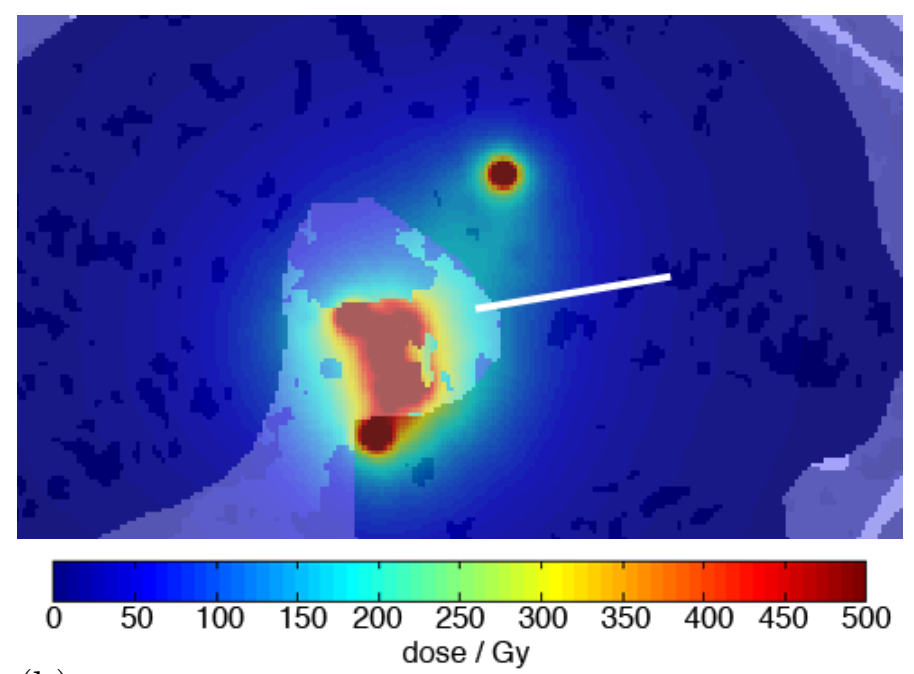

(b)

Figure 5.7: Composite images showing dose distributions and densities for a portion of computational phantoms for patient 5 derived from "fan" treated images using (a) CT-defined ("lung, air") and (b) nominal ("lung, air (nominal)") densities. The lines used for the profiles in figure 5.6 are shown.

rithm cannot be relied upon to sufficiently reduce all high CT numbers in the vicinity of the seeds such that erroneous bone tissue assignment is avoided in this region when generating computational phantoms. The median filter algorithm ("median") reduces metallic artifacts throughout the images and sufficiently reduces high CT numbers in the vicinity of the seeds such that erroneous bone tissue assignment is reduced when generating computational phantoms. However, while the median filter reduces noise in regions of muscle and adipose tissue, it removes regions of heterogeneity in lung 
tissue and can reduce the CT numbers for voxels representing bone tissue such that tissue mis-assignment occurs. Median filter techniques might be appropriate in treatment sites such as the prostate where seeds are located in regions that have less tissue heterogeneity. A combination of a fan beam virtual sinogram treatment followed by a simple threshold replacement ("fan+STR") sufficiently reduces high CT numbers due to seeds such that bone mis-assignment is avoided, while simultaneously reducing streaking artifacts and preserving tissue heterogeneities beyond the immediate vicinity of the seeds.

Full Monte Carlo calculations were performed for three of the six Mayo Clinic patients using computational phantoms generated by applying various tissue assignment schemes to the CT image sets. Constrained tissue assignment within lung contours reduces misassignment of voxels to bone in lung contours and so can be used to ameliorate the effects of suboptimal metallic artifact reduction. However, this improvement is contingent on the ${ }^{125}$ I seeds being found within the lung contour, which may not always be the case. Additionally, lung-constrained tissue assignment impedes the misassignment of voxels to adipose tissue, avoiding inaccuracies in dose distributions and dose metrics caused by the considerably lower mass attenuation and energy absorption coefficients of adipose tissue compared to lung tissue.111

A tissue assignment scheme that allows the assignment of muscle tissue within lung contours produces phantoms with resulting dose distributions that are approximately equal to those produced by a tissue assignment scheme that only allows lung and air to be assigned. This implies that, as long as the unknown atomic composition (and resulting mass attenuation and energy absorption coefficients) of lung fibrotic tissue does not differ considerably from that of lung or muscle, the presence of fibrotic tissue can be omitted from tissue assignment schemes without reducing the accuracy of calculated dose distributions. 
Computational phantoms with voxel densities assigned as the nominal density of the local voxel medium are not suitable for generating accurate dose distributions for lung brachytherapy patients as the nominal density of lung $\left(0.26 \mathrm{~g} / \mathrm{cm}^{3}\right)$ does not accurately represent the varied density of the lung tissue in the vicinity of the seeds.

The model that produced the most accurate Monte Carlo calculated dose distributions for lung brachytherapy patients (of the phantom models discussed) is a phantom generated by applying a tissue assignment scheme where only lung and air are assigned within lung contours to CT images that have been treated with a fan beam virtual sinogram algorithm followed by a simple threshold replacement algorithm. Since misassignment of tissues can considerably affect doses even when metallic artifacts are reduced, following the recommendation by the AAPM Task Group report 186 (Ref. 29) of using organ contours to guide tissue assignment will produce more realistic computational phantoms and consequently more accurate model-based dose calculations for brachytherapy in general. 


\section{Chapter 6}

\section{A Monte Carlo investigation of}

\section{lung brachytherapy treatment planning}

This chapter investigates avenues for an improved paradigm for lung brachytherapy treatment planning, drawing on the knowledge of media heterogeneity effects from chapters 4 and 5. Significant approximations are used in the standard practices for dosimetry and treatment planning for permanent implant lung brachytherapy. The area at risk of recurrence is determined intra-operatively and treatment planning only involves the use of a seed activity nomogram or look-up table to determine the seed spacing for the grid pattern mesh implant 1915 . Nomograms are typically calculated using the TG-43 formalism ${ }^{25} \mid 27$, i.e. doses are calculated assuming sources in a homogeneous water environment, with no interseed effects, and the point source approximation of the TG-43 formalism is often used.

In addition to ${ }^{125} \mathrm{I}$ seeds, ${ }^{131} \mathrm{Cs}$ seeds have also been used for lung brachytherapy and TG-43 calculated dose distributions have been compared between the two radionuclides ${ }^{21}$. However, having different emitted photon energy spectra, the deviations between TG-43 and model-based (including non-water media) calculated doses

will differ between ${ }^{125} \mathrm{I}$ and ${ }^{131} \mathrm{Cs}$ treatments due to differences in mass attenuation and energy absorption coefficients. 
Given the significant deviations between TG-43 and MC dose distributions found in chapter 4 , it is likely that there are significant differences between prescribed and delivered doses. Additionally, implantation of the planar mesh grid in patients leads to deformation of the plane of sources used for treatment planning and for prescription dose calculation. This deformation varies with implant site and, in conjunction with differences in the composition of surrounding tissues at different implant sites, will likely lead to large discrepancies between planned and delivered doses.

In this chapter, the accuracy of lung brachytherapy prescription doses calculated using TG-43 derived nomograms is investigated. Nomogram doses for planar source configurations are recalculated with BrachyDose using a variety of tissue models and these doses are compared to TG-43 calculated prescription doses. To investigate the changes in dose distributions between those of nomogram models and deformed implants in patients, a method of generating patient specific virtual implants is developed. Virtual implants are modelled for several example resection sites and Monte Carlo calculated dose distributions in a CT-derived patient phantom for these implants are compared to nomogram model dose distributions. Calculations are performed using both ${ }^{125} \mathrm{I}$ and ${ }^{131} \mathrm{Cs}$ seeds.

\subsection{Methods}

This chapter is comprised of two parts, described in the following sections: Section 6.1.1 investigates the effects of non-water media and interseed attenuation on prescription dose, assuming the planar seed configuration currently used for treatment planning. Section 6.1.2 considers deformation of the planar seed configuration, reflecting more realistic seed configurations encountered in patient treatments.

Calculations are for ${ }^{125} \mathrm{I}$ GE Healthcare/Oncura (Arlington Heights IL USA) 
Table 6.1: The mass attenuation ${ }^{30}$ and mass energy absorption coefficients (calculated with EGSnrc user-code g using XCOM) for 28 and $30 \mathrm{keV}$ photons (the approximate mean emitted photon energies of ${ }^{125} \mathrm{I}$ and ${ }^{131} \mathrm{Cs}$ respectively).

\begin{tabular}{ccccc}
\hline \hline \multirow{2}{*}{ Material } & \multicolumn{2}{c}{$\mu / \rho\left(\mathrm{cm}^{2} / \mathrm{g}\right)$} & \multicolumn{2}{c}{$\mu_{e n} / \rho\left(\mathrm{cm}^{2} / \mathrm{g}\right)$} \\
\cline { 2 - 5 } & $28 \mathrm{keV}$ & $30 \mathrm{keV}$ & $28 \mathrm{keV}$ & $30 \mathrm{keV}$ \\
\hline Adipose 2 & 0.348 & 0.321 & 0.116 & 0.095 \\
Air (TG-43) & 0.414 & 0.371 & 0.189 & 0.154 \\
Cortical bone & 1.617 & 1.353 & 1.317 & 1.069 \\
Lung - blood-filled & 0.443 & 0.398 & 0.201 & 0.164 \\
Muscle 2 & 0.439 & 0.395 & 0.198 & 0.162 \\
Water & 0.436 & 0.392 & 0.192 & 0.156 \\
\hline Ratio of lung to water & 1.016 & 1.015 & 1.047 & 1.051 \\
\hline \hline
\end{tabular}

model 6711 and ${ }^{131}$ Cs IsoRay (Richland WA USA) CS-1 Rev2 seeds. The ${ }^{131}$ Cs seed model is constructed using the dimensions reported by Rivard ${ }^{112}$. Initial photon energies and probabilities for ${ }^{125} \mathrm{I}$ and ${ }^{131} \mathrm{Cs}$ are sampled using the photon spectra quoted in TG-43U1 (Ref. 26) and the National Nuclear Data Center ${ }^{113}$ (NNDC), respectively. A subset of calculations were performed using the most recent NNDC ${ }^{125}$ I photon spectrum and results agreed within statistical uncertainty. The mean energies of photons emitted by ${ }^{125} \mathrm{I}$ and ${ }^{131} \mathrm{Cs}$ are approximately 28 and $30 \mathrm{keV}$, respectively. Mass attenuation and energy absorption coefficients at these energies for media used in this study are presented in table 6.1. Tissue elemental compositions are taken from Woodard and White ${ }^{\sqrt{85}}$ - see table 2.1 (page 19).

\subsubsection{Effect of non-water media on prescription dose}

In this part of the chapter, nomograms calculated using the TG-43 formalism are compared to BrachyDose simulations involving various tissue models. The phantoms used for BrachyDose calculations are $30 \times 30 \times 30 \mathrm{~cm}^{3}$; dose is scored in $1 \mathrm{~mm}^{3}$ voxels. Sources are arranged in rows of 10 seeds with $1 \mathrm{~cm}$ centre-to-centre between adjacent seeds within a row; four seed configurations with differing numbers of rows and row 
Table 6.2: The number of rows and spacing between rows for the four seed configurations considered (based on Johnson et al.155). In all four configurations, each row contains 10 seeds with $1 \mathrm{~cm}$ centre-to-centre distance.

\begin{tabular}{ccc}
\hline \hline Label & Number of rows & Row spacing $(\mathrm{cm})$ \\
\hline $\mathrm{A}$ & 6 & 0.8 \\
$\mathrm{~B}$ & 5 & 1.0 \\
$\mathrm{C}$ & 4 & 1.3 \\
$\mathrm{D}$ & 4 & 1.5 \\
\hline \hline
\end{tabular}

spacing are modelled (table 6.2) based on the nomogram reported by Johnson et al. ${ }^{15}$. The prescription dose is defined as the average of the doses to two points $5 \mathrm{~mm}$ above the plane of the implant: a point at the centre of the grid between rows and a point above a central row of seeds at the centre of the row (figure 6.1). Simulations are performed with $10^{9}$ histories to achieve statistical uncertainties of at most $0.4 \%$ for the prescription dose.

Using the analytical TG-43 point source formalism (TG- $43_{\text {point }}$ ) and BrachyDose derived TG-43 parameters for the ${ }^{125}$ I model 6711 (Ref. 77) and ${ }^{131}$ Cs CS-1 Rev2 seeds, the activity per seed required to produce the prescription dose (100 Gy) at the averaged prescription point is calculated. These activities are used to calculate the dose to the averaged prescription point for four phantom models (table 6.3): unit

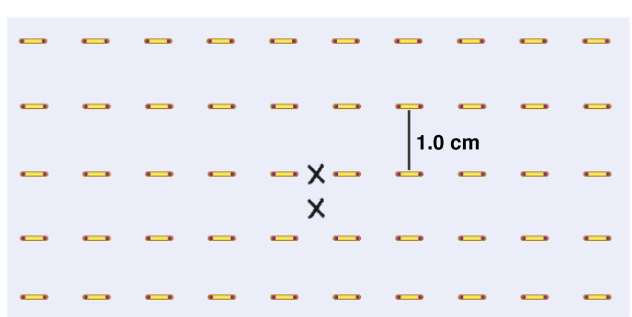

(a)

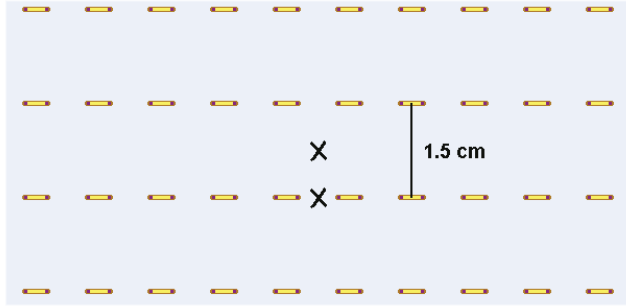

(b)

Figure 6.1: Diagram showing (a) the fives rows $(1.0 \mathrm{~cm}$ spacing) (configuration $\mathrm{B}$ ) and (b) four rows (1.5 cm spacing) (configuration D) seed configurations. The two points used to determine the prescription dose $(5 \mathrm{~mm}$ above the plane of the seeds) are marked with Xs. 
Table 6.3: Dose calculation descriptions and notation for prescription dose analysis. TG- $43_{\text {point }}$ and TG- $43_{\text {line }}$ are analytical calculations using the TG-43 formalism 27 and all other calculations are performed using BrachyDose. Water has unit density.

\begin{tabular}{|c|c|c|c|}
\hline phant. for photon transport & dose scoring medium & interseed effects & symbol \\
\hline \multicolumn{2}{|c|}{ - TG-43 point source model - } & no & TG- $43_{\text {point }}$ \\
\hline \multicolumn{2}{|c|}{ - TG-43 line source model - } & no & TG-43 line \\
\hline water & water & no & $\mathrm{TG}-43_{\operatorname{sim}}$ \\
\hline water & water & yes & $\mathrm{D}_{\mathrm{w}, \mathrm{w}}$ \\
\hline lung $\left(1.0 \mathrm{~g} / \mathrm{cm}^{3}\right)$ & water & yes & $\mathrm{D}_{\mathrm{w}, \mathrm{l}(1.0)}$ \\
\hline $\operatorname{lung}\left(1.0 \mathrm{~g} / \mathrm{cm}^{3}\right)$ & $\operatorname{lung}\left(1.0 \mathrm{~g} / \mathrm{cm}^{3}\right)$ & yes & $\mathrm{D}_{1,1(1.0)}$ \\
\hline $\operatorname{lung}\left(0.26 \mathrm{~g} / \mathrm{cm}^{3}\right)$ & water & yes & $\mathrm{D}_{\mathrm{w}, \mathrm{l}(0.26)}$ \\
\hline $\operatorname{lung}\left(0.26 \mathrm{~g} / \mathrm{cm}^{3}\right)$ & lung $\left(0.26 \mathrm{~g} / \mathrm{cm}^{3}\right)$ & yes & $\mathrm{D}_{1,1(0.26)}$ \\
\hline lung (CT derived den.) & water & yes & $\mathrm{D}_{\mathrm{w}, \mathrm{l}(\mathrm{CT})}$ \\
\hline lung (CT derived den.) & lung (CT derived den.) & yes & $\mathrm{D}_{1,1(\mathrm{CT})}$ \\
\hline
\end{tabular}

density water, unit density lung, nominal density $\left(0.26 \mathrm{~g} / \mathrm{cm}^{3}\right)$ lung, and lung with voxel densities defined by segments of lung from two patient CT data sets (denoted CT and CT2). The segments of lung in CT and CT2 are repeated to fill a volume of $30 \times 30 \times 30 \mathrm{~cm}^{3}$. Dose is generally scored in the local voxel medium. In a subset of calculations, dose to water voxels is scored to distinguish between the effects of voxel medium on photon energy fluence and absorbed dose, e.g., $\mathrm{D}_{\mathrm{w}, \mathrm{l}(0.26)}$ is dose to water voxels in nominal density lung. Calculations are performed for both ${ }^{125} \mathrm{I}$ and ${ }^{131} \mathrm{Cs}$ seeds.

\subsubsection{Effect of seed grid deformation and patient-defined media}

The combined effects of non-water tissues and deformation of the planar grid of seeds on dose distributions are investigated using CT images and organ structure data for a patient who underwent sublobar resection but not ${ }^{125} \mathrm{I}$ brachytherapy at the Mayo Clinic Rochester. As no brachytherapy sources were present during post- 


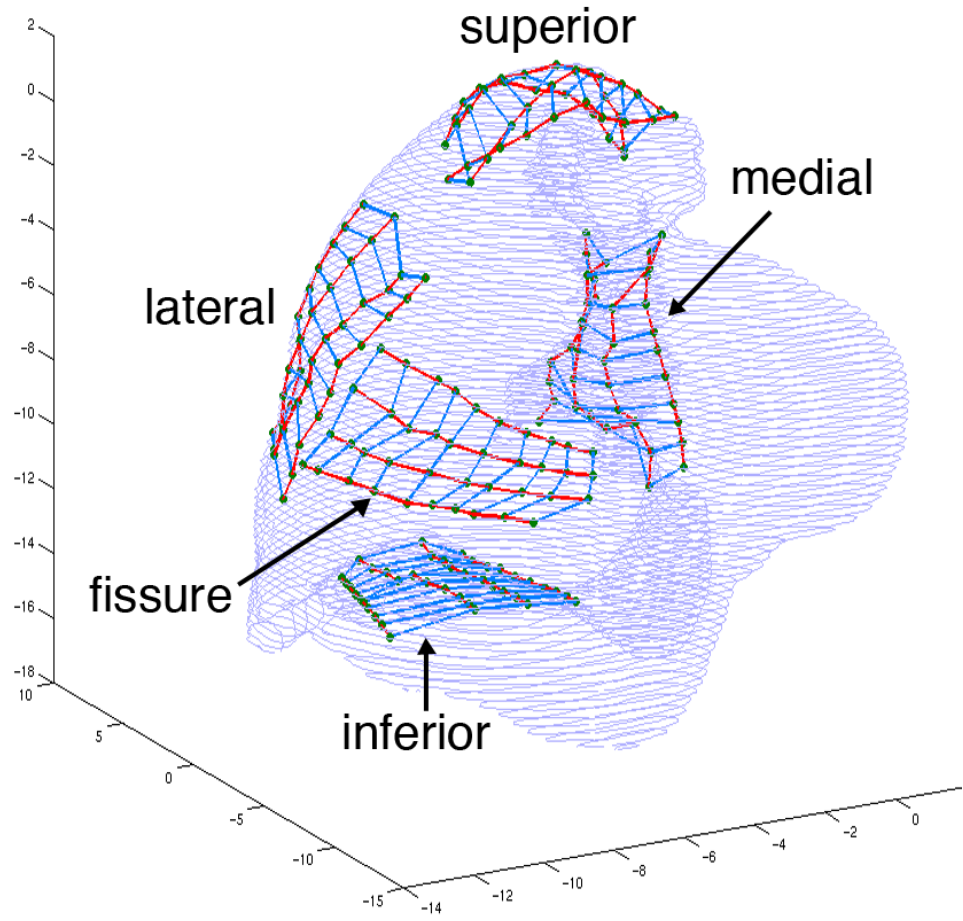

Figure 6.2: Illustration showing the 5 virtual implants and the contours of the right lung. Lines between sources indicate the 4 strands in an implant as well as the distance between seeds in adjacent strands. Axes are in centimetres.

treatment CT scans, the CT images do not suffer from streaking artifacts. Hence, resulting uncertainties in tissue assignment in the vicinity of artifacts ${ }^{111}$ are avoided in developing the computational phantoms used in the current study.

Virtual implants are created for five different sites for the patient's right lung using the lung contours: implants on the lateral, medial, superior and inferior surfaces of the lung and one implant approximating an implant on the horizontal fissure of the lung (figure 6.2). In practice, implants are placed on resection sites rather than undisturbed lung tissue; however, the five virtual implants illustrate the range of dose differences that are possible due to a variety of deformations and various tissues.

An algorithm was developed in MATLAB to generate the virtual implants by deforming the planar implant to conform to the points defining the lung structure 


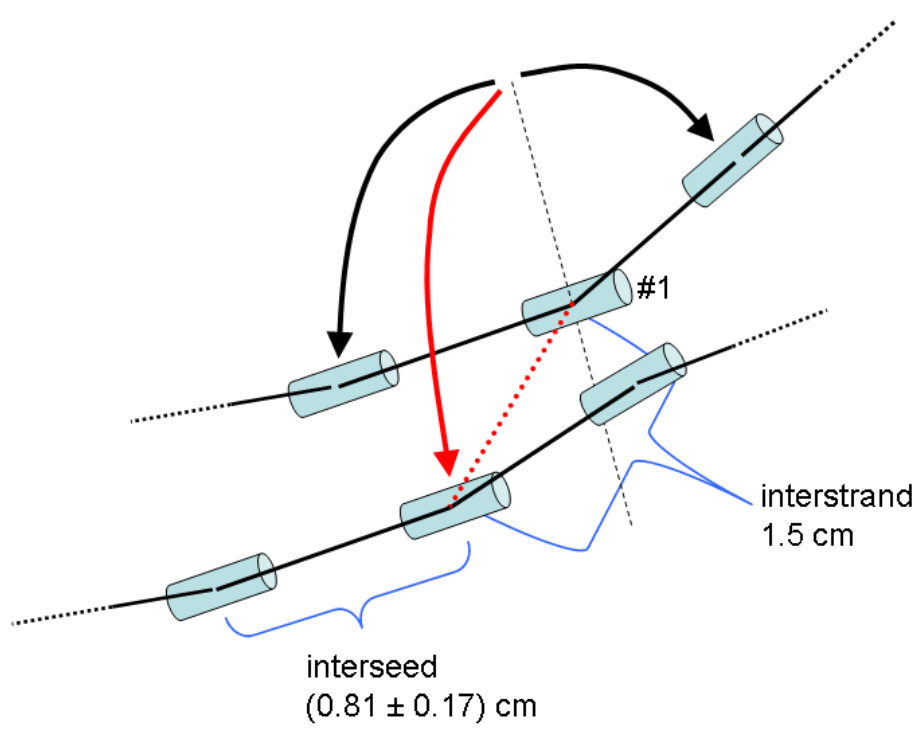

Figure 6.3: Illustration of the virtual implant creation algorithm. Starting from the seed labeled \#1, adjacent seeds in a strand are found by constraining the interseed distance to $0.81 \pm 0.17 \mathrm{~cm}$ and finding the minimum distance to lung contour points. The initial seed of an adjacent strand is found by constraining the distance from seed \#1 to $1.5 \mathrm{~cm}$ and then finding the minimum distance to lung contour points.

contours (figure 6.3). A point for the centre of the implant and the three direction vectors for the orientation of the implant are chosen. Seed positions are determined one strand at a time: starting with the central seed in a strand, adjacent positions are constrained by a specified interseed distance from the most recently determined seed and are determined by finding the point that has the minimum distance to the lung contour points. Once a single strand has been placed, adjacent strands are placed by moving across the implant from the central seed; first finding the point that has the minimum distance to the contour points that is also constrained by an interstrand distance and then placing the remaining seeds of that strand as before. A virtual suture line is needed for tabulating doses to volumes about the implant and is determined in the same manner as a strand of seeds in the centre of the implant.

Although treatment planning nomograms assume $1 \mathrm{~cm}$ spacing between seeds in 
a strand, interseed distance varies following implantation. Interseed distances of four Mayo Clinic patients treated with ${ }^{125}$ I lung mesh brachytherapy were calculated and are found to vary randomly with a mean value of $0.81 \mathrm{~cm}$ and standard deviation of $0.17 \mathrm{~cm}$. This distribution is used to randomly assign interseed distances when generating the virtual implants. An interstrand distance of $1.5 \mathrm{~cm}$ corresponding to configuration D in table 6.2 is used for the central seeds of each strand where distances between strands is constrained.

A phantom is created using CT data and a tissue assignment scheme that includes adipose, air, cortical bone, lung, and muscle, corresponding to the "simple" assignment scheme of chapter 4. This phantom is cropped to span approximately 30 $\mathrm{cm}$ in each direction centred on the right lung and, using original voxels sizes, has 239 voxels with a width of $0.125 \mathrm{~cm}$ in the anterior-posterior and lateral directions and 119 voxels with a width of $0.25 \mathrm{~cm}$ in the superior-inferior direction.

Monte Carlo calculated dose distributions for brachytherapy are sensitive to the conversion of CT image pixel values to atomic composition $\underline{48 / 108}$. By varying atomic composition, Landry et al. $\stackrel{42}{4}$ found up to $4 \%$ and $10 \%$ differences in the the minimum dose received by $90 \%$ of planning treatment volumes $\left(\mathrm{D}_{90}\right)$ for prostate and breast brachytherapy treatments, respectively. Chapters 4 and 5 presented significant differences in dose metrics for lung brachytherapy patients based on the use of different tissue assignment schemes. Consequently, the dose distributions presented in section 6.2.2 are dependent on the tissue assignment scheme chosen and they will be affected by errors in tissue assignment and so should be considered examples of the effects discussed.

Dose to water voxels in a water phantom with no interseed attenuation (TG$\left.43_{\text {sim }}\right)$, dose to water voxels in a CT-derived tissue phantom $\left(\mathrm{D}_{\mathrm{w}, \mathrm{m}(\mathrm{CT})}\right)$, and dose to the local voxel medium in a CT-derived tissue phantom $\left(\mathrm{D}_{\mathrm{m}, \mathrm{m}(\mathrm{CT})}\right)$ are calculated. 
For comparison, TG- $43_{\text {sim }}$ doses and $D_{1,1(0.26)}$ for the planar nomogram configuration D from table 6.2 (page 98) and section 6.1.1 (denoted as "planar D") as well as for a modified configuration where the in-row interseed spacing is $0.81 \mathrm{~cm}$ (denoted "planar (0.81) D") are calculated. Doses from ${ }^{125} \mathrm{I}$ and ${ }^{131} \mathrm{Cs}$ seeds are calculated and, in all cases, calculations are performed using the activity that would produce a dose of 100 Gy to the averaged prescription point of planar configuration D using TG- $43_{\text {sim }}$. For each of the different implant configurations, dose volume histograms are calculated for volumes consisting of voxels with centres that are within $5 \mathrm{~mm}$ and $20 \mathrm{~mm}$ of each implant's suture line (referred to as PTV05 and PTV20, respectively). Simulations are performed with $4 \times 10^{9}$ histories taking approximately $23 \mathrm{CPU}$ hours on a single 3.0 $\mathrm{GHz}$ Woodcrest core to achieve a maximum statistical uncertainty of less than $0.4 \%$ in PTV20 volumes.

\subsection{Results and discussion}

\subsubsection{Effect of non-water media on prescription dose}

Figure 6.4 shows the dose to the averaged prescription point calculated for seed configuration A (6 rows with $0.8 \mathrm{~cm}$ spacing) for each of the phantom models divided by the TG- $43_{\text {point }}$ calculated dose and using the same source activity; results are shown

for both ${ }^{125} \mathrm{I}$ and ${ }^{131} \mathrm{Cs}$ seeds. This dose ratio shows the deviation in the prescription dose for each dose calculation model compared to the TG- $43_{\text {point }}$ derived nomogram.

Focusing on the results in figure 6.4 for ${ }^{125} \mathrm{I}$, the TG-43 line calculation is $2 \%$ smaller than TG-43 point due largely to differences between the anisotropy function and anisotropy factor. TG- $43_{\text {sim }}$ is smaller than TG- $43_{\text {point }}$ by less than one percent; this difference is $2 \%$ when interseed attenuation is included $\left(\mathrm{D}_{\mathrm{w}, \mathrm{w}}\right)$. For $\mathrm{D}_{\mathrm{w}, \mathrm{l}(1.0)}$, the 


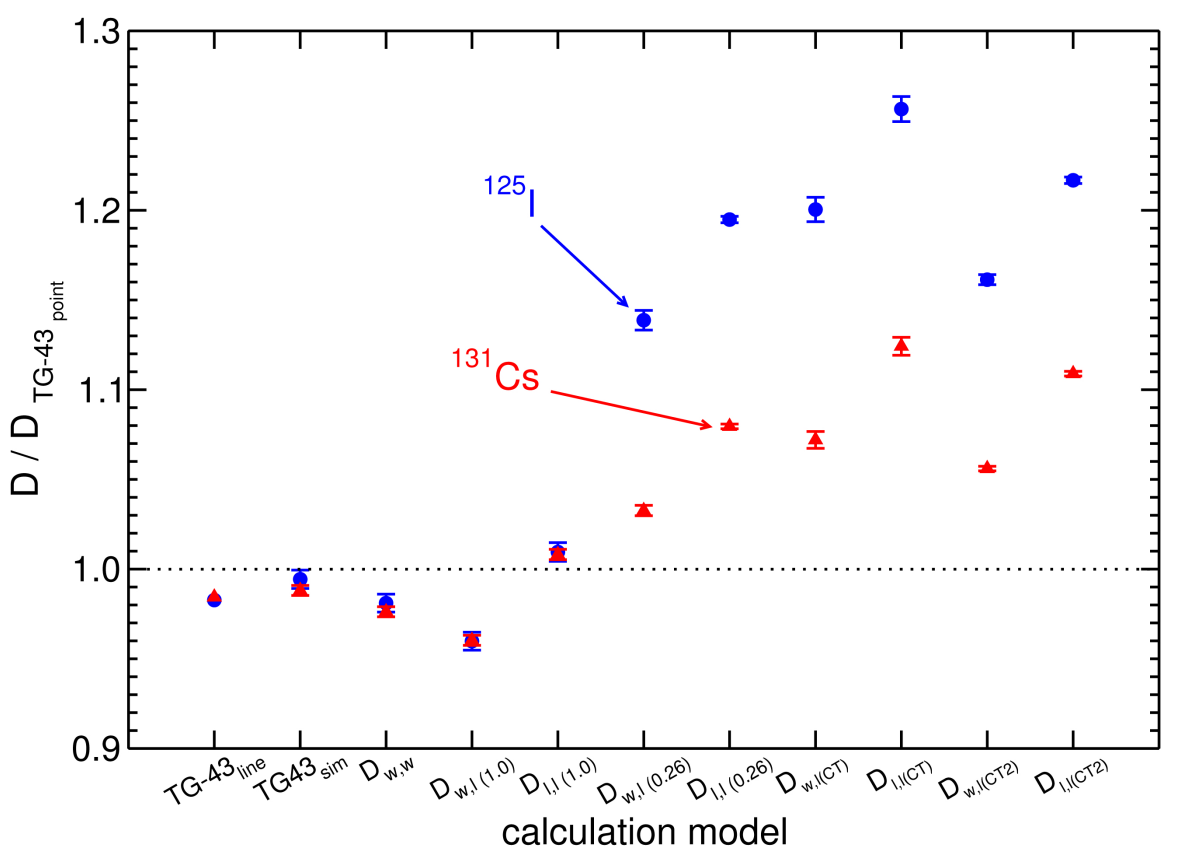

Figure 6.4: Doses to the averaged prescription point for the different models considered divided by the dose calculated by TG- $43_{\text {point }}$ for seed configuration A (6 rows, $0.8 \mathrm{~cm}$ row spacing).

larger mass attenuation coefficient of lung tissue compared to water $(1.6 \%$ for 28 $\mathrm{keV}$ photons) leads to a relative decrease in photon fluence and, consequently, the dose to the averaged prescription point is $4 \%$ smaller compared to TG- $43_{\text {point }}$. For $\mathrm{D}_{1,1(1.0)}$, this effect is compensated for by the larger mass energy absorption coefficient of lung (4.7\% for $28 \mathrm{keV}$ photons) resulting in a $1 \%$ larger dose than TG- $43_{\text {point }}$. For the calculations where the nominal density of lung is used $\left(\mathrm{D}_{\mathrm{w}, 1(0.26)}, \mathrm{D}_{1,1(0.26)}\right)$, the lower density results in reduced attenuation of photons compared to unit density lung or water and so the doses to the averaged prescription point are larger than TG- $43_{\text {point }}$ by $14 \%\left(\mathrm{D}_{\mathrm{w}, 1(0.26)}\right)$ and $19 \%\left(\mathrm{D}_{1,1(0.26)}\right)$. This effect is similarly observed in the calculations using CT-derived tissue densities with $20 \%$ and $25 \%$ larger doses for $\mathrm{D}_{\mathrm{w}, \mathrm{l}(\mathrm{CT})}$ and $\mathrm{D}_{\mathrm{l}, \mathrm{l}(\mathrm{CT})}$, respectively, and $16 \%$ and $22 \%$ larger doses for $\mathrm{D}_{\mathrm{w}, \mathrm{l}(\mathrm{CT} 2)}$ and $\mathrm{D}_{1,1(\mathrm{CT} 2)}$, respectively. The differences between $\mathrm{D}_{1,1(0.26)}, \mathrm{D}_{1,1(\mathrm{CT})}$, and $\mathrm{D}_{1,1(\mathrm{CT} 2)}$ are caused by differences in density and highlight the heterogeneous and patient-variable 
nature of the lung.

The trends of dose to the averaged prescription point with varying tissue model are less pronounced for ${ }^{131} \mathrm{Cs}$ than for ${ }^{125} \mathrm{I}$. While the mass attenuation coefficient ratio of lung to water is very similar for the mean emitted photon energies of ${ }^{125} \mathrm{I}$ and ${ }^{131} \mathrm{Cs}$, the mass attenuation coefficient of lung at the mean emitted photon energy of ${ }^{131} \mathrm{Cs}$ is approximately $10 \%$ smaller than that for ${ }^{125} \mathrm{I}$; thus, the photon fluence emitted from the ${ }^{131} \mathrm{Cs}$ sources is attenuated less than that from ${ }^{125} \mathrm{I}$. Since the average distance from a seed to the averaged prescription points remains the same, changes in photon fluence between water and nominal density lung or CT-density lung will be less pronounced for ${ }^{131} \mathrm{Cs}$. Additionally, since the mass energy absorption coefficient ratio of lung to water is similar for ${ }^{125} \mathrm{I}$ and ${ }^{131} \mathrm{Cs}$ spectra, differences between dose to lung voxels and dose to water voxels remain similar between ${ }^{125} \mathrm{I}$ and ${ }^{131} \mathrm{Cs}$ implants.

Figure 6.5 shows the isodose contours with ${ }^{125}$ I seeds comparing a TG- $43_{\text {sim }}$ calculation with an activity chosen to produce 100 Gy at the averaged prescription point and the same activity in a $\mathrm{D}_{1,1(0.26)}$ calculation for seed configuration C. The $\sim 20 \%$ difference near the averaged prescription point (fig. 6.4) is seen with the 100 Gy isodose line for TG-43 sim nearly overlapping the 125 Gy line for $\mathrm{D}_{1,1(0.26)}$. The relative difference in dose between the two calculations increases as the distance from the implant increases with the 10 Gy isodose line for TG-43 $3_{\text {sim }}$ nearly overlapping the 25 Gy line for $\mathrm{D}_{1,1(0.26)}$. Thus, beyond generally underestimating the dose to the averaged prescription point, TG-43 point also likely underestimates total lung doses and doses to organs at risk.

The differences in dose to the averaged prescription point seen in figure 6.4 can be generalized to all four seed configurations. However, the magnitude of the differences between TG-43 point and doses to nominal density lung and CT-density lung depend on seed configuration. The dose to the averaged prescription point for $\mathrm{D}_{1,1(0.26)}$ divided by 


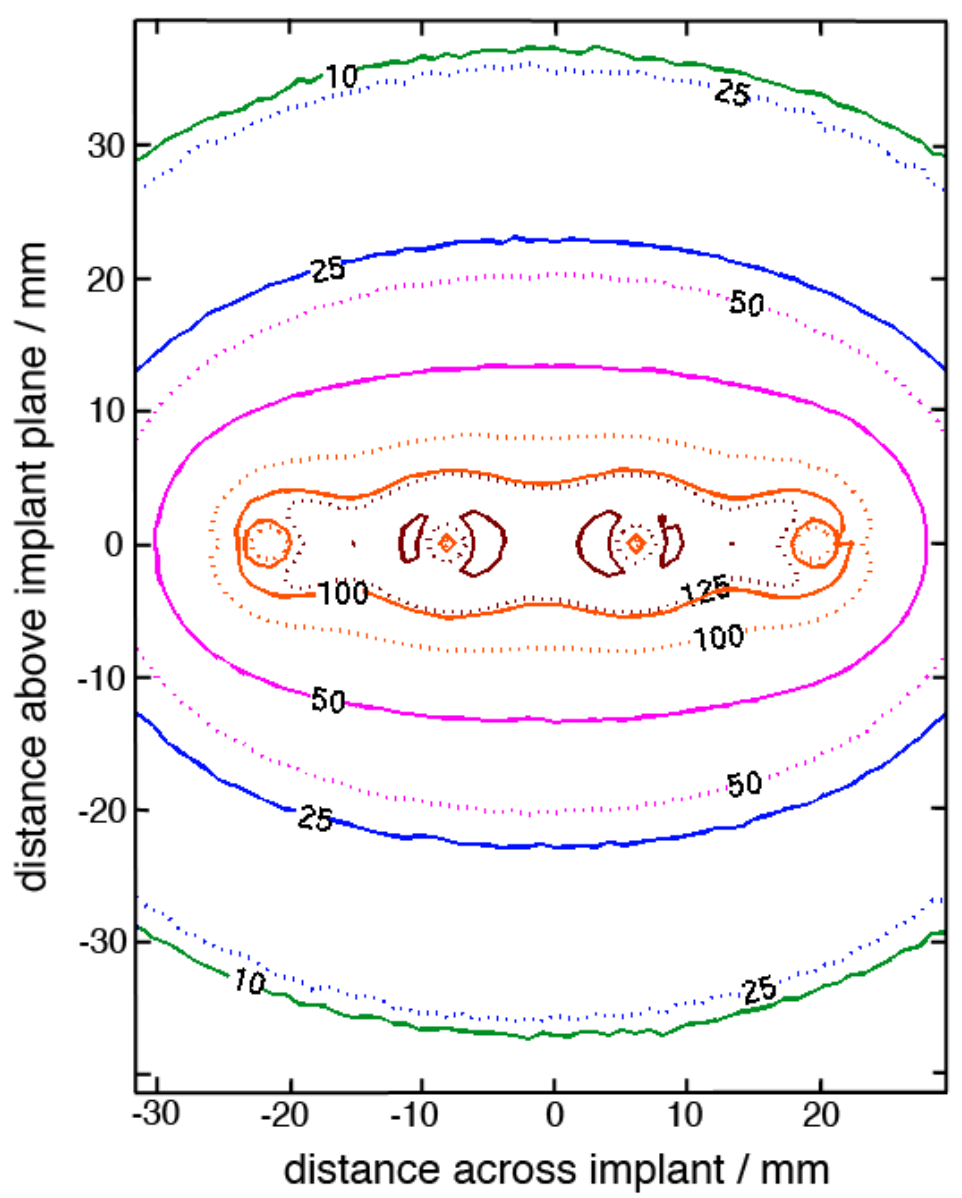

Figure 6.5: Isodose contours for the plane passing through the centre of the implant and orthogonal to the direction of the strands. Doses are calculated using ${ }^{125} \mathrm{I}$ seeds and seed configuration $\mathrm{C}$ (4 rows, $1.3 \mathrm{~cm}$ spacing) with TG$43_{\text {sim }}$ (solid lines) and $\mathrm{D}_{1,1(0.26)}$ (dotted lines). The activity is the same for both calculations and is chosen to produce 100 Gy at the averaged prescription point for TG- $43_{\text {sim }}$.

the dose calculated by TG- $43_{\text {point }}$ for all four seed configurations is shown in figure 6.6. For each configuration, the activity that would produce 100 Gy at the prescription point for TG- $43_{\text {point }}$ is used. For comparison, the data points have been arbitrarily shifted so that the points for configuration A are at 1.0. Figure 6.6 demonstrates that the deviation between TG- $43_{\text {point }}$ and $\mathrm{D}_{1,1(0.26)}$ calculated doses varies depending on the seed configuration. For more sparsely spaced seed configurations, the distance travelled by a photon to reach the averaged prescription point is generally larger 


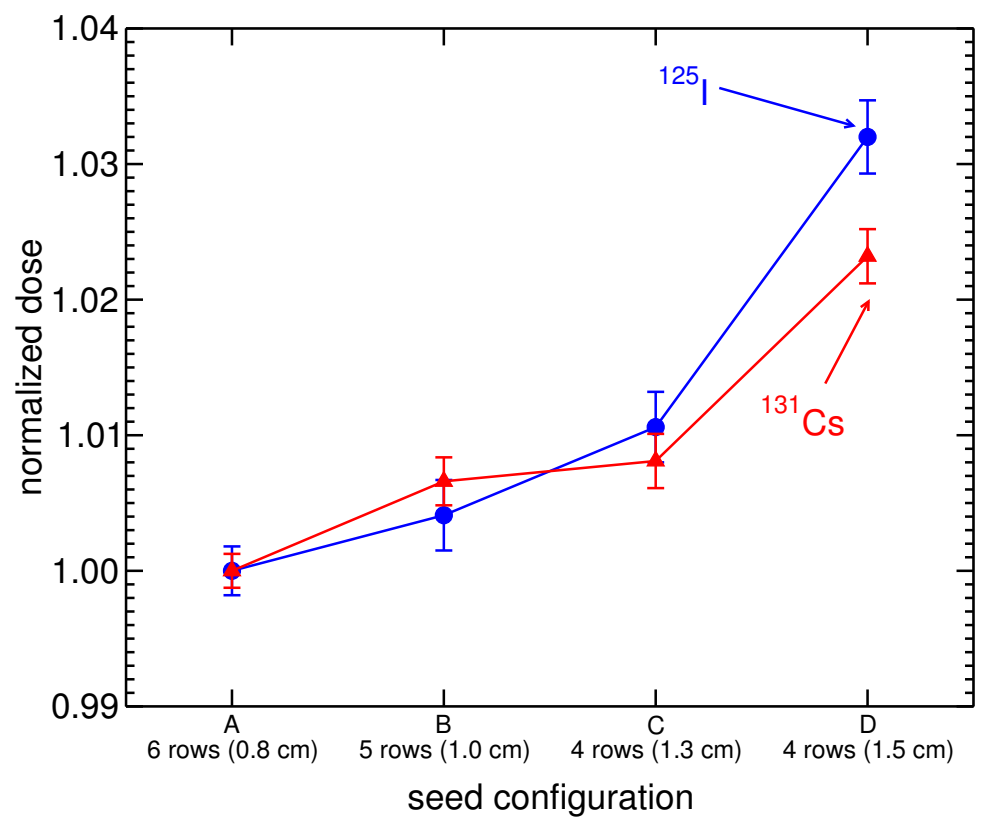

Figure 6.6: The dose to the averaged prescription point calculated using $\mathrm{D}_{1,1(0.26)}$ divided by to the dose calculated using TG- $43_{\text {point }}$ for each seed configuration. For comparison, the dose ratio points for each radionuclide have been arbitrarily shifted so that the points for configuration A are at 1.0. Lines connecting points are to aid the eye.

and so the difference in attenuation coefficients between nominal density lung and water leads to a greater photon fluence in lung at the averaged prescription point and, consequently, a larger dose for $\mathrm{D}_{1,1(0.26)}$ compared to TG-43 $3_{\text {point }}$. Thus, while two patients prescribed different implant configurations may seem to have the same dose to the averaged prescription point with a TG- $43_{\text {point }}$ calculation, in nominal density lung the doses may differ by more than $3 \%$ for ${ }^{125} \mathrm{I}$.

Table 6.4 shows a sample nomogram for ${ }^{125} \mathrm{I}$ and ${ }^{131} \mathrm{Cs}$ with the activity normalized to the prescription dose calculated for TG-43 $43_{\text {point }}$ or $\mathrm{D}_{1,1(0.26)}$. In other words, table 6.4 shows the activity that would produce a prescription dose of 1 Gy. The activity per seed calculated for $\mathrm{D}_{1,1(0.26)}$ is lower by more than $16 \%$ for ${ }^{125} \mathrm{I}$ and $7 \%$ for ${ }^{131}$ Cs from those calculated for TG- $43_{\text {point }}$. If TG- $43_{\text {point }}$ derived nomograms continue to be used for lung brachytherapy pre-planning then the clinician must be aware that 
Table 6.4: Nomograms for TG- $43_{\text {point }}$ and $\mathrm{D}_{1,1(0.26)}$ with activities normalized to prescription dose. Percent difference between activities per seed determined using TG- $43_{\text {point }}$ and $\mathrm{D}_{1,1(0.26)}$ calculations is shown.

\begin{tabular}{cccccc}
\hline \hline Label & $\begin{array}{c}\text { No. of } \\
\text { rows }\end{array}$ & $\begin{array}{c}\text { Row spacing } \\
(\mathrm{cm})\end{array}$ & $\begin{array}{c}\text { Activity per seed } \\
\text { norm. to Rx } \\
\text { for TG-43 } \\
(\mathrm{mCi} / \mathrm{Gy})\end{array}$ & $\begin{array}{c}\text { Activity per seed } \\
\text { norm. to Rx } \\
\text { for } \mathrm{D}_{1,1(0.26)} \\
(\mathrm{mCi} / \mathrm{Gy})\end{array}$ & $\begin{array}{c}\% \text { diff } \\
(\%)\end{array}$ \\
\hline A & 6 & 0.8 & 0.0032 & 0.0027 & 16 \\
$\mathrm{~B}$ & 5 & 1.0 & 0.0040 & 0.0033 & 17 \\
$\mathrm{C}$ & 4 & 1.3 & 0.0052 & 0.0043 & 17 \\
$\mathrm{D}$ & 4 & 1.5 & 0.0058 & 0.0047 & 18 \\
\hline & & & $131 \mathrm{Cs}$ & 7 \\
\hline $\mathrm{A}$ & 6 & 0.8 & 0.0082 & 0.0076 & 8 \\
$\mathrm{~B}$ & 5 & 1.0 & 0.0101 & 0.0093 & 8 \\
$\mathrm{C}$ & 4 & 1.3 & 0.0130 & 0.0120 & 9 \\
$\mathrm{D}$ & 4 & 1.5 & 0.0145 & 0.0131 & \\
\hline \hline
\end{tabular}

the relationship between the physical dose and the pre-planning dose is different for ${ }^{131} \mathrm{Cs}$ than it is for ${ }^{125} \mathrm{I}$. This should have implications for meta-analyses comparing clinical trials using the different radionuclides and may inform choice of the prescribed dose for future clinical trials.

The results described in this section are from simulations employing relatively simple computational phantoms involving few tissues/non-water media. In reality, different tissues may be found in the vicinity of an implant and these will vary based on implant site and patient anatomy. The dose distribution produced by an implant may differ from nomogram dose distributions because of these tissue differences, in addition to the deformation of the seed grid following implantation, and the effects of both are investigated in the following section. 


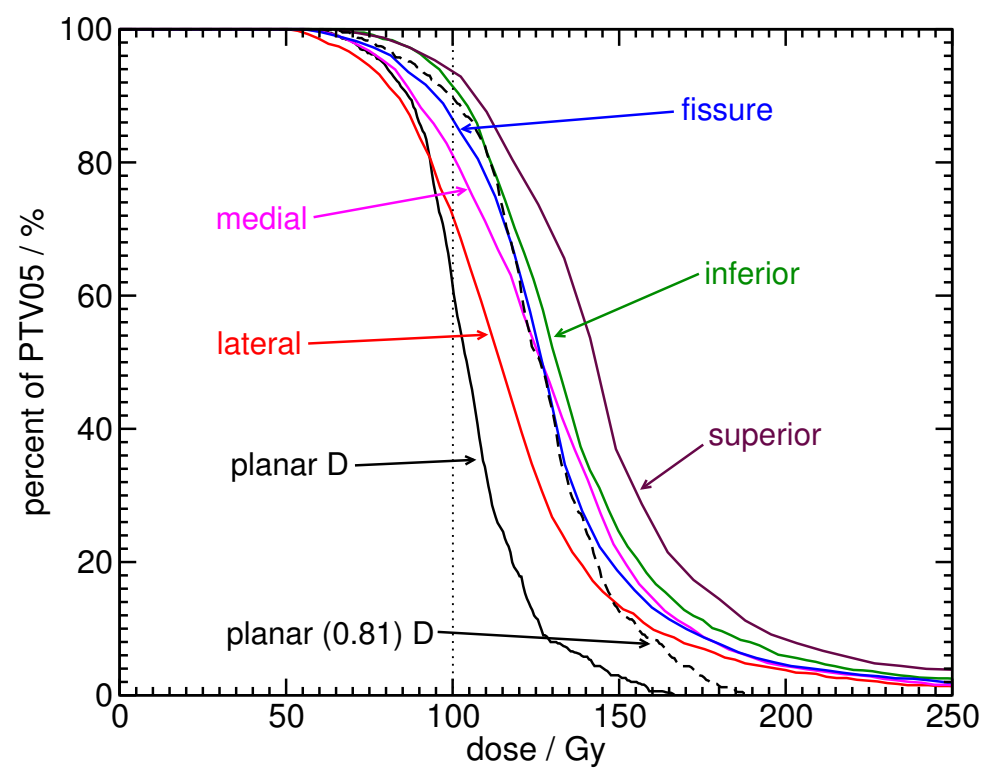

Figure 6.7: TG-43 $3_{\text {sim }}$ dose volume histograms for PTV05 for the five virtual implants (fissure, inferior, lateral, medial, and superior) and for the planar nomogram configuration D with interseed spacings $1.0 \mathrm{~cm}$ (planar D) and $0.81 \mathrm{~cm}$ (planar (0.81) D) ${ }^{125} \mathrm{I}$ seeds. Dose of $100 \mathrm{~Gy}$ is marked.

\subsubsection{Effect of seed grid deformation and patient-defined media}

Figure 6.7 presents dose volume histograms for PTV05 calculated for TG- $43_{\text {sim }}$ for the virtual implants compared to the planar nomogram configuration D and illustrates how delivered dose can change due to implant deformation. The dose distribution varies significantly based on the shape of the implant following deformation. The doses for the superior implant, which is the most curved of the virtual implants, deviate the most from those calculated using the planar configuration. In general, differences in DVHs can be understood by considering the severity of the implant deformation; implants that are quite deformed with smaller averaged distances from seeds to the suture line have larger doses.

Differences between DVHs calculated with the planar D seed configuration with 
$1.0 \mathrm{~cm}$ interseed spacing compared to calculations with the $0.81 \mathrm{~cm}$ interseed spacing (figure 6.7) illustrate how dose in the vicinity of the suture is highly sensitive to interseed spacing variations. Smaller interseed distances mean that activity is more concentrated around the suture line. As noted in section 6.1.2, investigation of Mayo Clinic patients found that average interseed distances for implants are consistently shorter than the $1 \mathrm{~cm}$ distance used for planning. This suggests that the current nomogram-derived pre-planning underestimates post-planned doses, independent of dose calculation model or variations in implant deformation.

Table 6.5 shows the percentage of PTV05 and PTV20 receiving at least 100 Gy $\left(\mathrm{V}_{100}\right)$ and table 6.6 shows the minimum dose received by $90 \%$ of PTV05 and PTV20 $\left(\mathrm{D}_{90}\right)$ for the planar D implants as well as the virtual implants for TG-43 $3_{\mathrm{sim}}, \mathrm{D}_{\mathrm{w}, \mathrm{m}(\mathrm{CT})}$, and $\mathrm{D}_{\mathrm{m}, \mathrm{m}(\mathrm{CT})}\left(\mathrm{D}_{\mathrm{l}, \mathrm{l}(0.26)}\right.$ for planar implants $)$ for ${ }^{125} \mathrm{I}$ and ${ }^{131} \mathrm{Cs}$ seeds. There is a large variation in both $\mathrm{V}_{100}$ and $\mathrm{D}_{90}$ between the different virtual implants. $\mathrm{V}_{100}$ and $\mathrm{D}_{90}$ tend to be larger for $\mathrm{D}_{\mathrm{w}, \mathrm{m}(\mathrm{CT})}$ compared to $\mathrm{TG}-43_{\text {sim }}$ since the presence of lower density lung tissue attenuates less than unit density water. However, for $\mathrm{D}_{\mathrm{m}, \mathrm{m}(\mathrm{CT})}$, the effect of the mass energy absorption coefficients of the various tissues in the treatment volumes (air, lung, adipose, muscle, and bone) often results in a lower dose than $\mathrm{D}_{\mathrm{w}, \mathrm{m}(\mathrm{CT})}$. The exception to this is the "fissure" virtual implant which is surrounded exclusively by lung tissue. The results shown in tables 6.5 and 6.6 suggest that doses in the suture vicinity cannot be accurately predicted by a TG-43 calculation with a single seed configuration; doses depend on both implant deformation and tissues in the treatment volume.

The interplay between the effect of deformation and patient media can be illustrated by comparing the "fissure" and "superior" virtual implants. Figure 6.8 shows dose volume histograms for ${ }^{125}$ I seeds for PTV05 of the "fissure" virtual implant and represents an example where this interplay is relatively simple. This implant is rela- 
Table 6.5: The percentage of the volume that receives at least $100 \mathrm{~Gy}\left(\mathrm{~V}_{100}\right)$ for PTV05 and PTV20 for the virtual implants and also some planar implants. Results are reported for TG-43 $3_{\text {sim }}, D_{\mathrm{w}, \mathrm{m}(\mathrm{CT})}$, and $\mathrm{D}_{\mathrm{m}, \mathrm{m}(\mathrm{CT})}\left(\mathrm{D}_{\mathrm{l}, \mathrm{l}(0.26)}\right.$ for planar implants) with ${ }^{125} \mathrm{I}$ and ${ }^{131} \mathrm{Cs}$ seeds.

\begin{tabular}{|c|c|c|c|c|c|c|}
\hline \multirow[b]{2}{*}{ Implant } & \multicolumn{3}{|c|}{ PTV05 } & \multicolumn{3}{|c|}{ PTV20 } \\
\hline & $\mathrm{TG}-43_{\mathrm{sim}}$ & $\mathrm{D}_{\mathrm{w}, \mathrm{m}(\mathrm{CT})}$ & $\begin{array}{l}\mathrm{D}_{\mathrm{m}, \mathrm{m}(\mathrm{CT})} \\
\left(\mathrm{D}_{1, \mathrm{l}(0.26)}\right)\end{array}$ & TG- $43_{\text {sim }}$ & $\mathrm{D}_{\mathrm{w}, \mathrm{m}(\mathrm{CT})}$ & $\begin{array}{l}\mathrm{D}_{\mathrm{m}, \mathrm{m}(\mathrm{CT})} \\
\left(\mathrm{D}_{1,1(0.26)}\right) \\
\end{array}$ \\
\hline \multicolumn{7}{|c|}{${ }^{125} \mathrm{I}$} \\
\hline planar D & 63 & - & $(93)$ & 17 & - & $(31)$ \\
\hline planar (0.81) D & 90 & - & $(96)$ & 27 & - & $(40)$ \\
\hline fissure & 80 & 93 & 95 & 25 & 37 & 40 \\
\hline inferior & 92 & 95 & 87 & 27 & 30 & 28 \\
\hline lateral & 74 & 81 & 75 & 25 & 33 & 34 \\
\hline medial & 83 & 88 & 72 & 30 & 34 & 32 \\
\hline superior & 94 & 89 & 84 & 37 & 39 & 42 \\
\hline \multicolumn{7}{|c|}{${ }^{131} \mathrm{Cs}$} \\
\hline planar D & 68 & - & $(86)$ & 18 & - & $(25)$ \\
\hline planar (0.81) D & 90 & - & (94) & 28 & - & $(34)$ \\
\hline fissure & 89 & 92 & 94 & 28 & 33 & 37 \\
\hline inferior & 93 & 93 & 86 & 28 & 29 & 27 \\
\hline lateral & 77 & 79 & 74 & 27 & 30 & 32 \\
\hline medial & 85 & 87 & 70 & 32 & 33 & 31 \\
\hline superior & 96 & 91 & 86 & 39 & 38 & 42 \\
\hline
\end{tabular}

tively flat and, as it is along the horizontal fissure, it is surrounded by lung tissue. For $\mathrm{D}_{\mathrm{w}, \mathrm{m}(\mathrm{CT})}$, the volume (PTV05) receives a larger dose compared to TG- $43_{\text {sim }}$ because of a larger photon fluence due to decreased attenuation in lower density lung (relative to water). For $\mathrm{D}_{\mathrm{m}, \mathrm{m}(\mathrm{CT})}$, the dose again increases because lung has a slightly larger mass energy absorption coefficient than water (see table 6.1).

In contrast, the combined effect of deformation and different tissues is more complicated for the "superior" implant, as demonstrated in the dose volume histograms for PTV05 and PTV20 for this implant with ${ }^{125}$ I seeds shown in figure 6.9. As the "superior" implant is directly below the clavicle, PTV05 for this implant contains approximately $8 \%$ bone, $19 \%$ muscle, $15 \%$ adipose, and $58 \%$ lung by volume. Focusing on PTV05, the percentage of the volume with doses near 100 Gy is smaller for 
Table 6.6: The minimum dose $(\mathrm{Gy})$ that $90 \%$ of the volume receives $\left(\mathrm{D}_{90}\right)$ for PTV05 and PTV20 for the virtual implants and also some planar implants. Results are reported for TG- $43_{\text {sim }}, D_{\mathrm{w}, \mathrm{m}(\mathrm{CT})}$, and $\mathrm{D}_{\mathrm{m}, \mathrm{m}(\mathrm{CT})}\left(\mathrm{D}_{\mathrm{l}, \mathrm{l}(0.26)}\right.$ for planar implants) with ${ }^{125} \mathrm{I}$ and ${ }^{131} \mathrm{Cs}$ seeds.

\begin{tabular}{|c|c|c|c|c|c|c|}
\hline \multirow[b]{2}{*}{ Implant } & \multicolumn{3}{|c|}{ PTV05 } & \multicolumn{3}{|c|}{ PTV20 } \\
\hline & TG- $43_{\text {sim }}$ & $\mathrm{D}_{\mathrm{w}, \mathrm{m}(\mathrm{CT})}$ & $\begin{array}{l}\mathrm{D}_{\mathrm{m}, \mathrm{m}(\mathrm{CT})} \\
\left(\mathrm{D}_{1,1(0.26)}\right) \\
\end{array}$ & TG- $43_{\text {sim }}$ & $\mathrm{D}_{\mathrm{w}, \mathrm{m}(\mathrm{CT})}$ & $\begin{array}{l}\mathrm{D}_{\mathrm{m}, \mathrm{m}(\mathrm{CT})} \\
\left(\mathrm{D}_{\mathrm{l}, \mathrm{l}(0.26)}\right)\end{array}$ \\
\hline \multicolumn{7}{|c|}{${ }^{125} \mathrm{I}$} \\
\hline planar D & 87 & - & $(105)$ & 12 & - & (19) \\
\hline planar (0.81) D & 99 & - & $(120)$ & 13 & - & $(22)$ \\
\hline fissure & 88 & 106 & 111 & 14 & 23 & 23 \\
\hline inferior & 104 & 109 & 84 & 18 & 22 & 19 \\
\hline lateral & 85 & 89 & 76 & 15 & 15 & 11 \\
\hline medial & 90 & 97 & 76 & 18 & 21 & 20 \\
\hline superior & 107 & 99 & 87 & 31 & 23 & 21 \\
\hline \multicolumn{7}{|c|}{${ }^{131} \mathrm{Cs}$} \\
\hline planar D & 87 & - & $(96)$ & 16 & - & $(22)$ \\
\hline planar (0.81) D & 99 & - & (109) & 18 & - & $(25)$ \\
\hline fissure & 97 & 104 & 109 & 18 & 27 & 26 \\
\hline inferior & 104 & 104 & 81 & 22 & 24 & 21 \\
\hline lateral & 86 & 87 & 76 & 20 & 19 & 14 \\
\hline medial & 93 & 95 & 75 & 22 & 24 & 23 \\
\hline superior & 115 & 104 & 90 & 24 & 18 & 16 \\
\hline
\end{tabular}

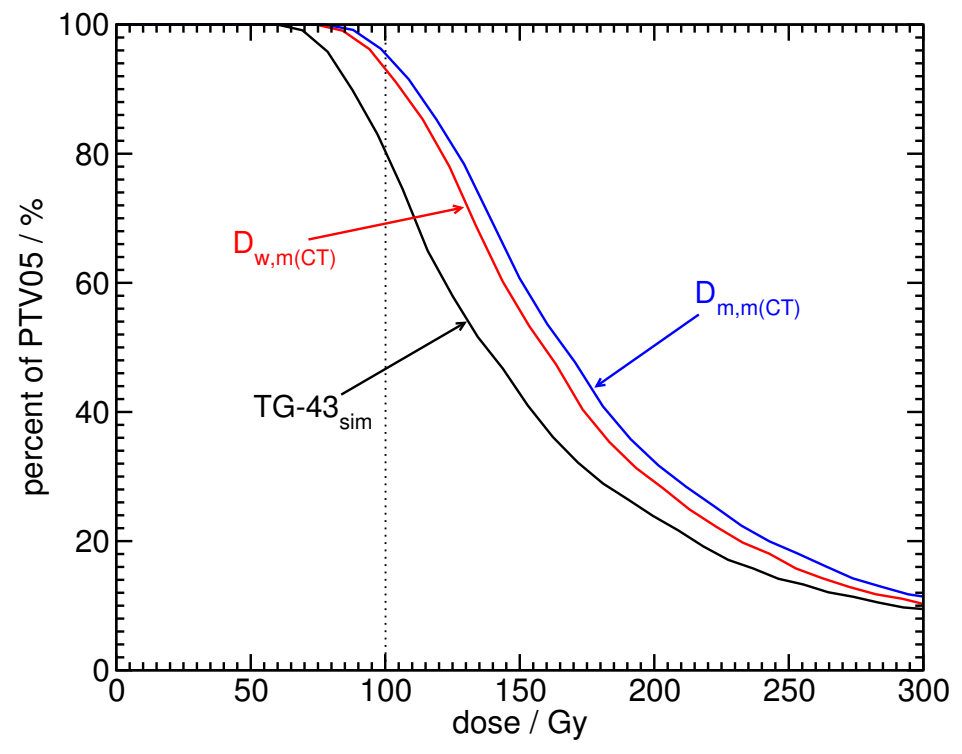

Figure 6.8: Dose volume histograms for PTV05 of "fissure" virtual implant for TG- $43_{\text {sim }}, \mathrm{D}_{\mathrm{w}, \mathrm{m}(\mathrm{CT})}$, and $\mathrm{D}_{\mathrm{m}, \mathrm{m}(\mathrm{CT})}$ and ${ }^{125} \mathrm{I}$ seeds. Dose of 100 Gy is marked. 


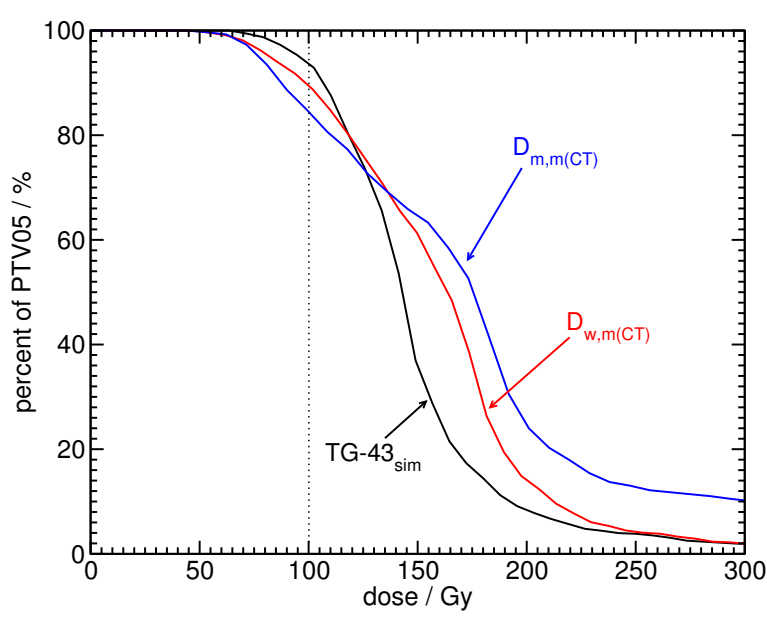

(a)

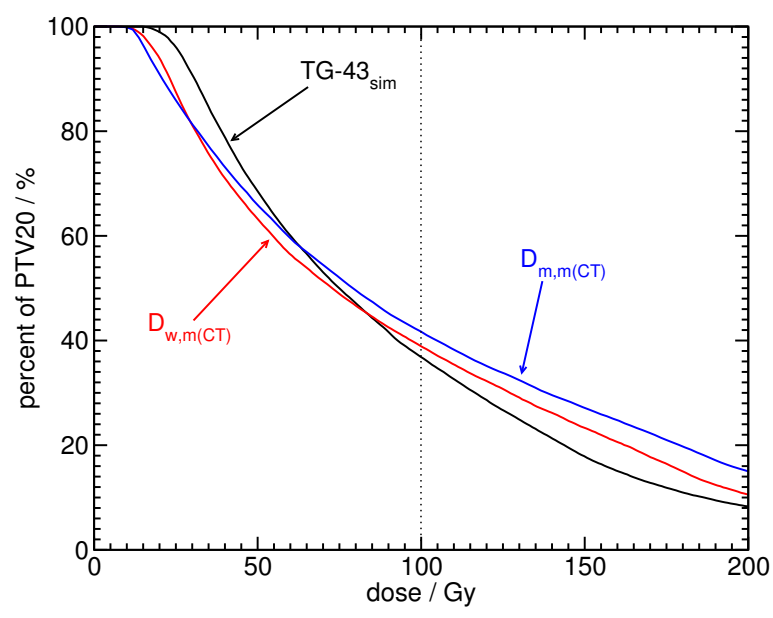

(b)

Figure 6.9: Dose volume histograms for (a) PTV05 and (b) PTV20 of the "superior" virtual implant for TG- $43_{\mathrm{sim}}, \mathrm{D}_{\mathrm{w}, \mathrm{m}(\mathrm{CT})}$, and $\mathrm{D}_{\mathrm{m}, \mathrm{m}(\mathrm{CT})}$ and ${ }^{125} \mathrm{I}$ seeds. Dose of 100 Gy is marked.

$\mathrm{D}_{\mathrm{w}, \mathrm{m}(\mathrm{CT})}$ than for TG- $43_{\text {sim }}$, while the percentage of the volume with doses between 100 and 250 Gy is larger for $\mathrm{D}_{\mathrm{w}, \mathrm{m}(\mathrm{CT})}$. The lower density of lung and adipose means that those voxels that had TG-43 sim doses near 100 Gy (voxels that were near the edge of PTV05) are receiving larger doses for $\mathrm{D}_{\mathrm{w}, \mathrm{m}(\mathrm{CT})}$. The very large mass energy absorption coefficient of bone causes $\mathrm{D}_{\mathrm{m}, \mathrm{m}(\mathrm{CT})}$ in voxels containing bone to be dramatically larger than $\mathrm{D}_{\mathrm{w}, \mathrm{m}(\mathrm{CT})}$. Consequently, the DVH for $\mathrm{D}_{\mathrm{m}, \mathrm{m}(\mathrm{CT})}$ is further reduced at approximately 100 Gy and a larger percentage of voxels have a dose exceeding 200 Gy compared to $\mathrm{D}_{\mathrm{w}, \mathrm{m}(\mathrm{CT})}$. The larger mass energy absorption coefficient of lung also serves to increase $\mathrm{D}_{\mathrm{m}, \mathrm{m}(\mathrm{CT})}$, but the effect is less dramatic than that of bone. The phenomena observed for PTV05 are reflected in the shape of the DVH for PTV20. However, since PTV20 contains a larger proportion of lung and muscle tissues, the relative magnitude of DVH differences is smaller for PTV20 than for PTV05.

The differences between the results for TG- $43_{\mathrm{sim}}, \mathrm{D}_{\mathrm{w}, \mathrm{m}(\mathrm{CT})}$, and $\mathrm{D}_{\mathrm{m}, \mathrm{m}(\mathrm{CT})}$ in the dose volume histograms for the "superior" implant may be further understood by considering figure 6.10 which presents dose and density profiles along a line passing 
near the seeds. In the left-most portion of the profile, $\mathrm{D}_{\mathrm{w}, \mathrm{m}(\mathrm{CT})}$ and $\mathrm{D}_{\mathrm{m}, \mathrm{m}(\mathrm{CT})}$ do not differ significantly because mass energy absorption coefficients for muscle and water are similar. However, both $\mathrm{D}_{\mathrm{w}, \mathrm{m}(\mathrm{CT})}$ and $\mathrm{D}_{\mathrm{m}, \mathrm{m}(\mathrm{CT})}$ are significantly larger than TG- $43_{\text {sim }}$ since the photons traveled through lung tissue to reach the muscle in the $\mathrm{D}_{\mathrm{w}, \mathrm{m}(\mathrm{CT})}$ and $\mathrm{D}_{\mathrm{m}, \mathrm{m}(\mathrm{CT})}$ calculations, resulting in greater photon fluences. In the portion of the profile that passes through lung, $\mathrm{D}_{\mathrm{m}, \mathrm{m}(\mathrm{CT})}$ is larger than $\mathrm{D}_{\mathrm{w}, \mathrm{m}(\mathrm{CT})}$ which is in turn larger than TG- $43_{\text {sim }}$. In adipose tissue, the smaller mass energy absorption coefficient causes a significant drop in $\mathrm{D}_{\mathrm{m}, \mathrm{m}(\mathrm{CT})}$ compared to $\mathrm{D}_{\mathrm{w}, \mathrm{m}(\mathrm{CT})}$. The voxels of bone cause spikes of high dose for $\mathrm{D}_{\mathrm{m}, \mathrm{m}(\mathrm{CT})}$ because of the large mass energy absorption coefficient of bone; the presence of bone results in decreases in $\mathrm{D}_{\mathrm{w}, \mathrm{m}(\mathrm{CT})}$ compared to $\mathrm{TG}-43_{\text {sim }}$ in the adjacent muscle voxels (due to the large attenuation coefficient in bone).

\subsection{Conclusions}

Significant differences in the dose to the averaged prescription point are observed between TG-43 $43_{\text {point }}$ calculations and model-based dose calculations with more realistic phantoms which include non-water media. These discrepancies are primarily due to differences in density and attenuation coefficients for the non-water media present compared to water. In general, the TG-43 approach (TG-43 point) significantly underestimates doses. Additionally, treatments with different numbers of sources and different source positions which have the same dose to the averaged prescription point according to TG-43 $3_{\text {point }}$ calculations may have different doses with model-based dose calculations.

Observed dose differences between TG-43 and model-based dose calculations vary in magnitude for the two radionuclides considered, with larger differences for 


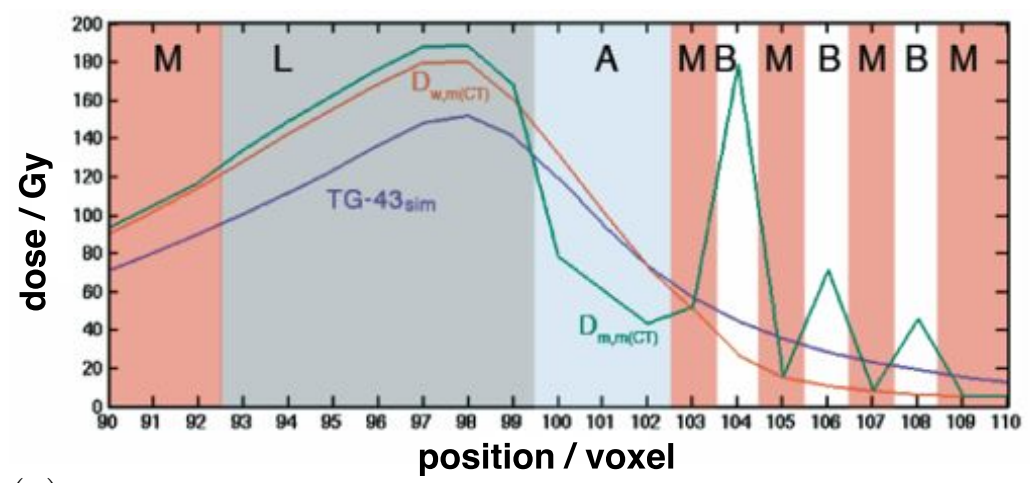

(a)

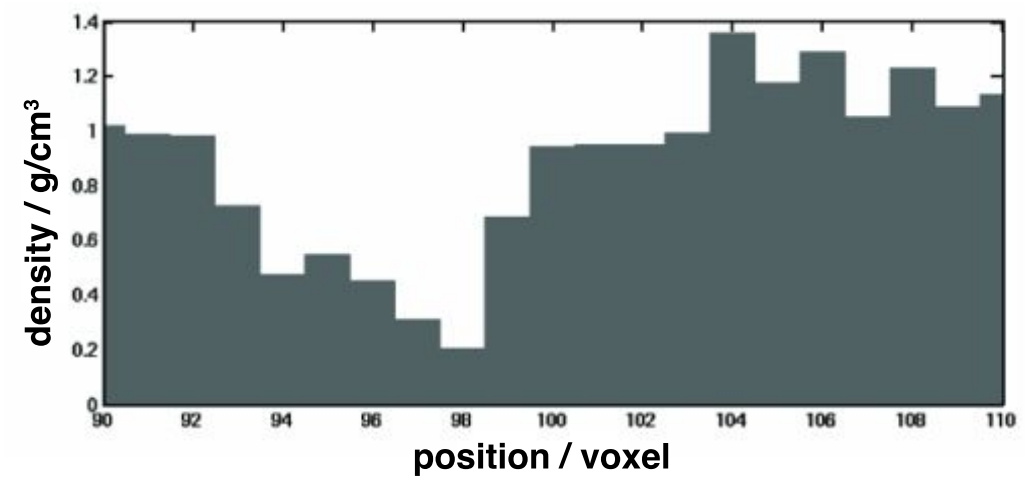

(b)

(c)

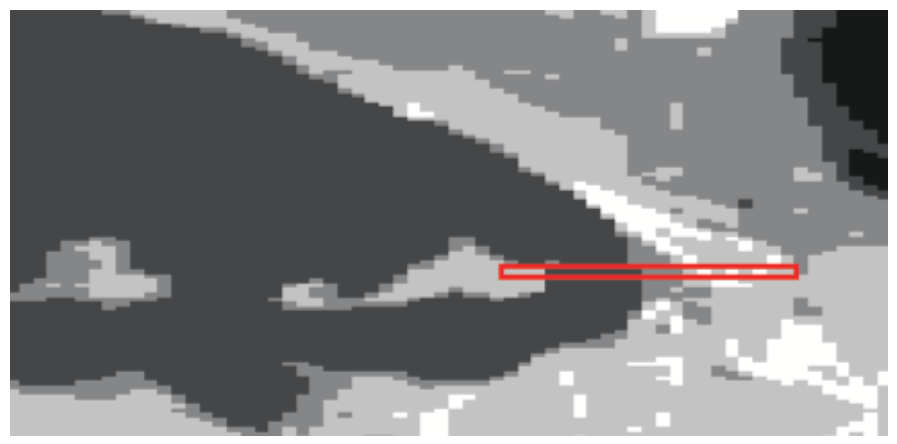

Figure 6.10: Dose (a) and density (b) profiles along the line shown in (c). Doses are calculated using the "superior" virtual implant for TG-43 $3_{\text {sim }}, \mathrm{D}_{\mathrm{w}, \mathrm{m}(\mathrm{CT})}$, and $\mathrm{D}_{\mathrm{m}, \mathrm{m}(\mathrm{CT})}$. Shading behind the dose profile curves denotes the tissue found in each voxel. From left to right the segments are: muscle (M), lung (L), adipose (A), and alternating muscle and bone. (c) Coronal slice segment of the patient phantom with an outline showing the voxels used for the profiles. Each shade corresponds to a tissue; from darkest to lightest: air, lung, adipose, muscle, and bone. The profile passes near the seeds of the "superior" virtual implant. 
treatment planning and evaluation.

Deviations in patient-specific dose distributions from those calculated using the TG-43 formalism with planar implants depend on patient geometry, the exact deformation of the implant and the tissue assignment scheme used. The dose to an averaged pre-planning reference point is insufficient for accurately producing desired dose distributions in patients. In addition to the radionuclide dependence, dose is sensitive to the actual tissues in the vicinity of the implant and to implant deformation. Although different treatments may have identical pre-plans based on the nomograms in current clinical use, actual dose distributions may differ greatly. Given the significant deviations between model-based dose calculations observed in this chapter and chapters 4 and 5 , patient-specific model-based dose calculations may be necessary to more accurately determine the dose dependence of clinical endpoints.

A more accurate method to predict dose distributions than is possible with the current treatment planning paradigm may be necessary in order to apply any knowledge of the dose dependence of clinical endpoints. The results presented here suggest that such a treatment planning approach must account, at least approximately, for seed grid deformation and the effects of non-water media. The development of a more accurate treatment planning approach could involve a virtual implant method similar to the one used in this work. Such an approach would require further research to approximately predict the shape of the surgical suture (and hence, the implant) from pre-implant CT images. The orientation and shape of sutures depends on tumour size, location, and the resection method. A study of pre- and post-implant CT images for patients receiving lung brachytherapy might shed light on common characteristics of implants and might be valuable for creating predictive models of implant geometries. 


\section{Chapter 7}

\section{Monte Carlo calculated doses to treatment volumes and organs at risk for permanent implant lung brachytherapy}

While treatments for permanent implant lung brachytherapy have currently only been reported for ${ }^{125} \mathrm{I}$ and ${ }^{131} \mathrm{Cs}$ implants ${ }^{21114}$, the use of ${ }^{169} \mathrm{Yb}$ surgical staples is being investigated (Ref. 22), ${ }^{103} \mathrm{Pd}$ has been used in the treatment of stage III NSCLC ${ }^{115}$, and the use of novel radionuclides continues to be investigated for brachytherapy in general $[116$.

Yang and Rivard ${ }^{[55}$ calculated dose distributions using a simplified lung geometry with monoenergetic point sources of various energies and found considerable differences between TG-43 and Monte Carlo calculated doses. This thesis has presented considerable differences between TG- 43 and Monte Carlo calculated dose distributions for post-treatment patient specific calculations (chapter 4), pre-planning prescription dose calculations (chapter 6), as well as differences between patient specific Monte Carlo calculated dose distributions using different radionuclides (chapter 6). Additionally, chapter 6 showed that the deformation of implants and variations in tissues in the vicinity of implants were found to considerably affect dose distributions. These effects varied in magnitude for ${ }^{125} \mathrm{I}$ and ${ }^{131} \mathrm{Cs}$ seeds, suggesting that doses to pa- 
tient treatment volumes are sensitive to the choice of radionuclide, independent of prescription doses.

The literature for permanent implant lung brachytherapy has made only sparse mention of doses to healthy tissues. A study of the dose to intrathoracic organs was reported $\frac{117}{17}$ but dose calculations were performed using the TG-43 formalism. Additionally, while ${ }^{125}$ I lung brachytherapy has been reported to be safe with virtually no increased morbidity 17 , some patients may be excluded as candidates for brachytherapy or prescription doses may be lowered due to concern for healthy tissues $^{21}$. Doses to healthy tissues will be sensitive to the photon energy spectra of the radionuclide used and differences in dose due to photon energy spectra are likely to be misrepresented by water-based calculations. Consequently, accurate model-based dose calculations are important for understanding how to safely and effectively adopt new radionuclides and to better inform the current use of ${ }^{125} \mathrm{I}$ and ${ }^{131} \mathrm{Cs}$ sources in lung brachytherapy.

This chapter investigates dosimetry for permanent implant lung brachytherapy considering a range of source energies and various implant sites in the lung. Doses to treatment volumes as well as the ipsilateral lung, heart, and aorta are studied. Monte Carlo calculations are performed for a variety of virtual implants generated using patient lung contours (see section 6.1.2, page 99) and using a computational phantom derived from patient CT and organ-contour data. Dose distributions are calculated for ${ }^{103} \mathrm{Pd},{ }^{125} \mathrm{I}$, and ${ }^{131} \mathrm{Cs}$ seeds as well as for 50 and $100 \mathrm{keV}$ monoenergetic point sources in the computational phantom and in a homogeneous water phantom with no interseed attenuation to approximate TG-43 calculations. Dose metrics for the heart, aorta, and lung as well as virtual treatment volumes are compared. 


\subsection{Methods}

Two Monte Carlo dose calculation methods are used in this chapter. In the first, denoted TG- $43_{\text {sim }}$, dose is calculated in a homogeneous water phantom with no interseed attenuation. In the second, denoted $\mathrm{D}_{\mathrm{m}, \mathrm{m}(\mathrm{CT})}$ (dose to medium in medium), dose is calculated in a patient-derived computational phantom with dose scored to the local voxel medium. The computational phantom is derived from CT and organ contour data of a Mayo Clinic Rochester patient who underwent sublobar resection but not ${ }^{125} \mathrm{I}$ brachytherapy. To increase computational efficiency, the CT image of $512 \times 512 \times 126$ voxels with voxel dimensions of $1.25 \times 1.25 \times 2.5 \mathrm{~mm}^{3}$ is cropped and resampled to an image of $300 \times 300 \times 126$ voxels with voxel dimensions of $1.73 \times 1.73 \times 2.5 \mathrm{~mm}^{3}$ before subsequent conversion to the computational phantom. An organ-constrained tissue assignment scheme (figure 7.1) is used to assign voxel media with elemental compositions taken from Woodard and White ${ }^{85}$ (see table 2.1. page 19): air and lung are assigned within lung contours; aorta and blood are assigned within the aorta contours; heart is assigned within the heart contours; and air, adipose, muscle and bone are assigned elsewhere. The mass density of each voxel is assigned using the CT to mass density calibration shown in table 4.2 (page 52 ). Table 7.1 gives the mass attenuation and absorption coefficient ratios of water to each tissue.

Seed positions for calculations are determined using the virtual implant generating algorithm described in chapter 6 (page 99) and the same source positions are used for all source types. Grid pattern mesh implants composed of 4 strands of 10 seeds each with interstrand distances of $1.5 \mathrm{~cm}$ and a distribution of interseed distances of $0.8 \pm 0.17 \mathrm{~cm}$ (chosen to approximate deviations from planned $1 \mathrm{~cm}$ distances in patients) are modelled: starting with the centre position of the implant, adjacent seed 
Table 7.1: Mass attenuation ${ }^{30}$ and energy absorption coefficient ratios (calculated with the EGSnrc user-code g) of water to tissues for the tissues used in this study. Values are shown for the mean emitted photon energies of ${ }^{103} \mathrm{Pd}(21 \mathrm{keV}),{ }^{125} \mathrm{I}$ $(28 \mathrm{keV}),{ }^{131} \mathrm{Cs}(30 \mathrm{keV})$, and 50 and $100 \mathrm{keV}$.

\begin{tabular}{ccccccc}
\hline \hline \multirow{2}{*}{ Ratio } & \multirow{2}{*}{ Tissue } & \multicolumn{5}{c}{ Photon energy $(\mathrm{keV})$} \\
\cline { 3 - 6 } & Adipose 2 & 1.391 & 1.254 & 1.224 & 1.073 & 1.014 \\
& Air (TG-43) & 1.040 & 1.053 & 1.058 & 1.087 & 1.105 \\
& Aorta & 0.967 & 0.977 & 0.980 & 1.000 & 1.010 \\
$\left(\frac{\mu}{\rho}\right)_{\text {tissue }}^{\text {water }}$ & Blood - whole & 0.964 & 0.974 & 0.977 & 0.997 & 1.007 \\
& Cortical bone & 0.214 & 0.270 & 0.290 & 0.539 & 0.914 \\
& Heart - blood-filled & 0.969 & 0.977 & 0.980 & 0.998 & 1.007 \\
& Lung - blood-filled & 0.976 & 0.983 & 0.986 & 1.000 & 1.007 \\
& Muscle 2 & 0.988 & 0.993 & 0.994 & 1.004 & 1.009 \\
& Adipose 2 & 1.690 & 1.651 & 1.638 & 1.372 & 1.047 \\
& Air (TG-43) & 1.020 & 1.014 & 1.013 & 1.031 & 1.095 \\
$\left(\frac{\mu_{e n}}{\rho}\right)^{\text {water }}{ }_{\text {tissue }}$ & Aorta & 0.943 & 0.935 & 0.933 & 0.947 & 0.998 \\
& Blood - whole & 0.941 & 0.934 & 0.934 & 0.944 & 0.995 \\
& Cortical bone & 0.152 & 0.146 & 0.146 & 0.181 & 0.555 \\
& Heart - blood-filled & 0.948 & 0.939 & 0.941 & 0.949 & 0.994 \\
& Lung - blood-filled & 0.958 & 0.953 & 0.953 & 0.965 & 0.998 \\
& Muscle 2 & 0.974 & 0.968 & 0.968 & 0.975 & 1.002 \\
\hline \hline
\end{tabular}




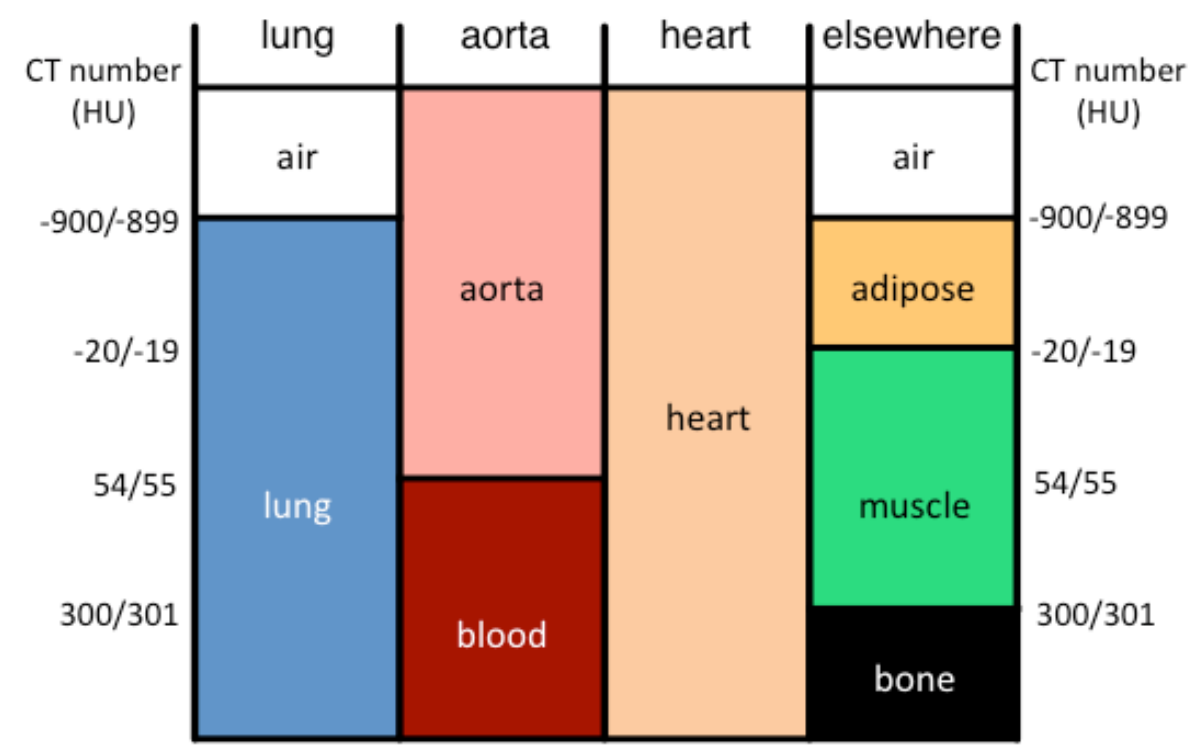

Figure 7.1: A diagram of the tissue assignment scheme used. CT number boundaries in HU are shown on the right and left. Each column is a separate scheme that is applied to voxels found outside of contours ("elsewhere") or within lung, aorta, or heart contours. For example, a voxel with a CT number of $60 \mathrm{HU}$ is assigned blood within the aorta contour but to muscle if it is not within any contour.

positions are determined one-by-one by finding the position of closest approach to the lung contours that is constrained by some (interseed/interstrand) distance. Suture lines for each implant are defined by the midpoints between adjacent seed positions of the two inner strands. Sixteen virtual implants are generated with central points defined by the intersection points of equally spaced radial lines in the axial plane starting from an arbitrary central point and the contours of the right lung. This produces implant positions arranged around the surface of the right lung that are conformed to the surface of the lung. The axial plane on which the central points of the sixteen implants are defined is chosen to approximately intersect the centres of the heart and aorta contours. A seventeenth implant is modelled within the right lung to approximate an implant placed on the horizontal fissure. The implants have differing deformations, surrounding tissues, and distances from the heart and aorta. 
Figure 7.2 shows points indicating the centres of each virtual implant overlaid on a slice of the patient CT image, as well as contours for the heart and aorta. As an example, figure 7.3 shows implant number 1 and the contours of the right lung.

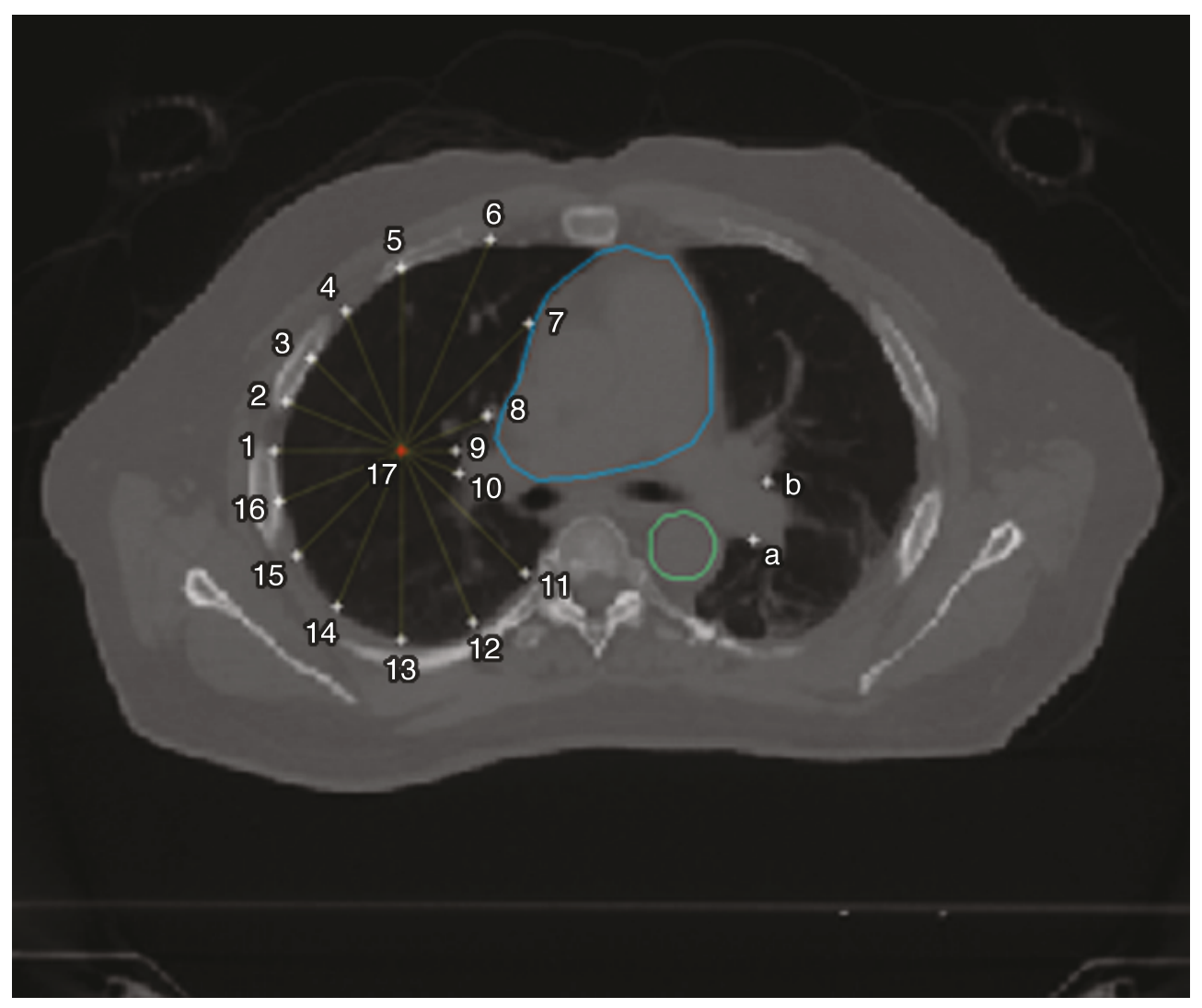

Figure 7.2: A slice of the CT image for the Mayo Clinic patient view from an inferior position. Heart (upper) and aorta (lower) contours are shown. The positions of the centres of the virtual implants in the right lung are marked and numbered 1-17. Implant 17 is modelled as an implant placed on the horizontal fissure. Two implant positions in the left lung (labelled "a" and "b") are also indicated.

Five source models are considered for this study: ${ }^{103} \mathrm{Pd}$ (mean emitted photon energy of the radionuclide, $\overline{E_{\gamma}}=21 \mathrm{keV}$ ) Theragenics Co. (Buford Georgia USA) TheraSeed 200, ${ }^{125} \mathrm{I}\left(\overline{E_{\gamma}}=28 \mathrm{keV}\right)$ GE Healthcare/Oncura (Arlington Heights IL USA) model 6711, ${ }^{131} \mathrm{Cs}\left(\overline{E_{\gamma}}=30 \mathrm{keV}\right)$ IsoRay (Richland WA USA) CS-1 Rev2, as well as 50 and $100 \mathrm{keV}$ monoenergetic point sources. Monoenergetic sources are used 


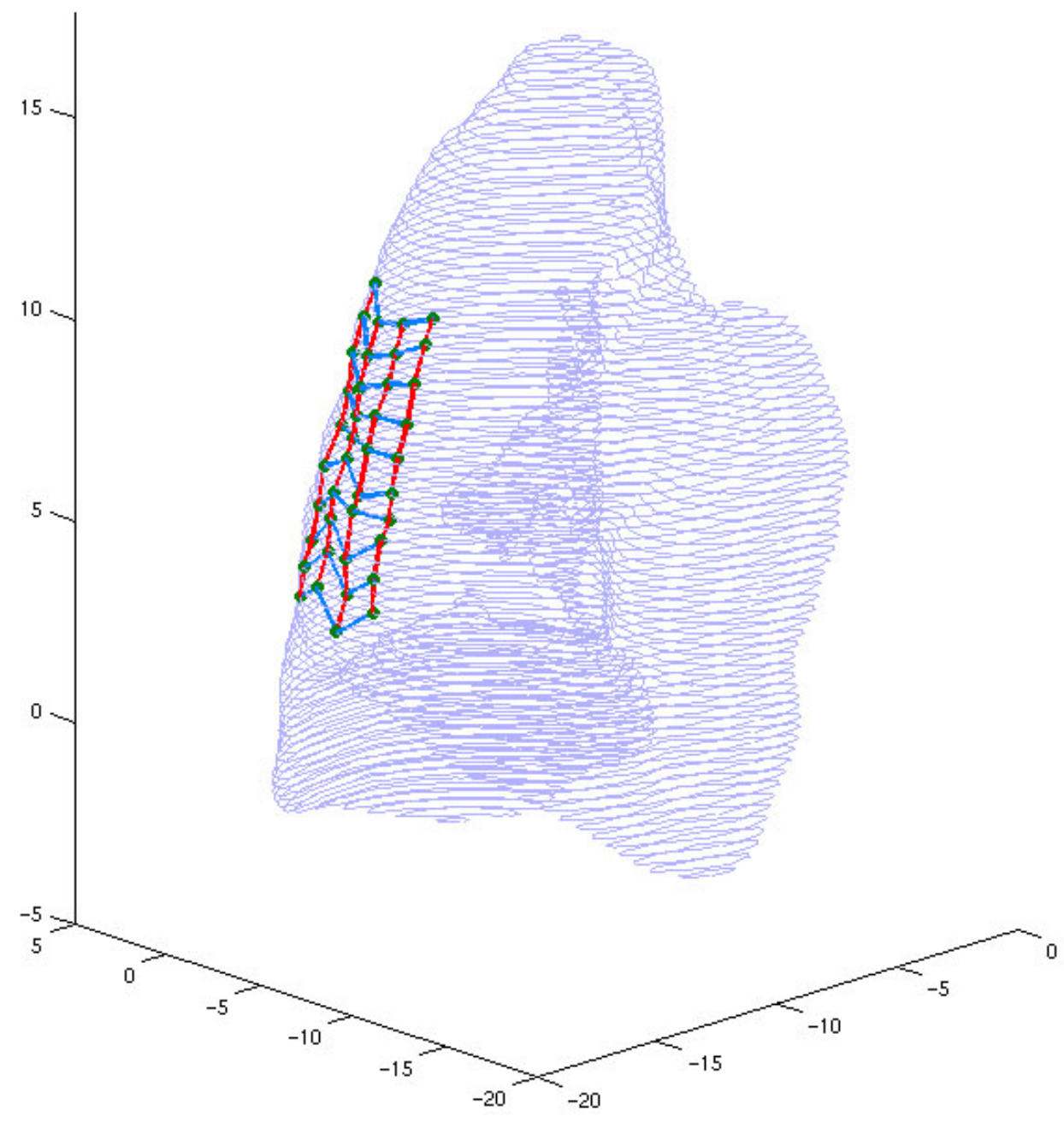

Figure 7.3: An illustration of virtual implant no. 1 and the contours of the right lung. Lines between sources indicate the four strands in the implant as well as the distance between seeds in adjacent strands. Axes are in $\mathrm{cm}$.

to extend the results of ${ }^{103} \mathrm{Pd},{ }^{125} \mathrm{I}$, and ${ }^{131} \mathrm{Cs}$ sources to intermediate energies and also to approximate ${ }^{169} \mathrm{Yb}\left(\overline{E_{\gamma}}=93 \mathrm{keV}\right)$ sources.

Dose distributions for each source type are normalized to produce a TG- $43_{\text {sim }}$ calculated prescription dose of 100 Gy for the usual planar implant $\frac{15}{15}$ : the prescription dose is defined as the average of the doses at two points $5 \mathrm{~mm}$ above a planar grid of $4 \times 10$ seeds with interstrand and interseed distances of 1.5 and $1 \mathrm{~cm}$, respectively. The two points are a point at the centre of the grid between rows and a point above 
a central row of seeds at the centre of the row (see chapter 6, page 97). An arbitrary prescription dose of $100 \mathrm{~Gy}$, while unrealistic for sources other than ${ }^{125} \mathrm{I}$, facilitates comparison between source types and results in each dose also being equivalent to the percentage of an arbitrary prescription dose $\left(\% \mathrm{D}_{\mathrm{Rx}}\right)$.

Dose metrics are reported for heart, aorta, and ipsilateral (right) lung volumes as well as for PTV05 and PTV20 treatment volumes which are defined as $5 \mathrm{~mm}$ and $20 \mathrm{~mm}$ expansions of suture lines, respectively. The whole heart volume is contoured and the aorta contours delineate $7 \mathrm{~cm}$ of the descending aorta. Mean doses to the aorta and heart, as well as the heart volume that receives at least $5 \mathrm{~Gy}\left(\mathrm{~V}_{5}\right)$ are calculated. Dose volume histograms (DVHs) for the fissure implants (position no. 17) and mean doses for all of the implants are calculated for the ipsilateral (right) lung volume. For PTV05 and PTV20, the minimum doses received by the hottest 90\% of the volumes $\left(\mathrm{D}_{90}\right)$ and the percentages of the volumes that receive at least $100 \mathrm{~Gy}$ $\left(\mathrm{V}_{100}\right.$ or, equivalently, $\left.\mathrm{V}_{\mathrm{D}_{\mathrm{Rx}}}\right)$ and $200 \mathrm{~Gy}\left(\mathrm{~V}_{200}\right)$ are calculated. Calculations, taking approximately $240 \mathrm{CPU}$ hours on a single $3.0 \mathrm{GHz}$ Woodcrest core are performed with $5 \times 10^{10}$ histories to achieve a maximum statistical uncertainty of $0.4 \%$ in PTV20 volumes for ${ }^{103} \mathrm{Pd}$ calculations (with lower uncertainties achieved for other sources).

\subsection{Results and Discussion}

\subsubsection{Doses to treatment volumes}

$\mathrm{D}_{90}$ for PTV05 and PTV20 for the various implants and radionuclides can be seen in figure 7.4. Values of $\mathrm{D}_{90}$ for $\mathrm{D}_{\mathrm{m}, \mathrm{m}(\mathrm{CT})}$ vary between implants and range between $40-$ 150 Gy and 7 - 45 Gy for PTV05 and PTV20, respectively. The range of $\mathrm{D}_{90}$ values for TG-43 $3_{\text {sim }}$ is comparable but somewhat smaller: 35 - 120 Gy for PTV05 and 13 - 
50 Gy for PTV20. Values of $\mathrm{D}_{90}$ generally have closer agreement between source types for PTV05 than for PTV20.

The means and standard deviations of $\mathrm{D}_{90}$ across the seventeen virtual implants for each source and for $\mathrm{D}_{\mathrm{m}, \mathrm{m}(\mathrm{CT})}$ and $\mathrm{TG}-43_{\text {sim }}$ are given in table 7.2 . The standard deviation in $\mathrm{D}_{90}$ across the seventeen implants is larger for ${ }^{103} \mathrm{Pd}$ than for the other source types for both PTV05 and PTV20, and both calculation methods $\left(\mathrm{D}_{\mathrm{m}, \mathrm{m}(\mathrm{CT})}\right.$ and TG-43 $\left.3_{\operatorname{sim}}\right)$. Lower-energy photons have a shorter range and undergo less scatter than higher-energy photons; hence, doses within treatment volumes are more sensitive to implant deformation for the lower-energy sources. This generally results in larger values for the standard deviation in $\mathrm{D}_{90}$ across implants for the lower-energy sources.

Table 7.2: The mean and standard deviations of $\mathrm{D}_{90}(\mathrm{~Gy})$ for the seventeen virtual implants for PTV05 and PTV20 for each source type and dose calculation method. See figure 7.4 for individual values.

\begin{tabular}{|c|c|c|c|c|c|}
\hline \multirow[b]{2}{*}{ Source } & \multirow[b]{2}{*}{ Dose calc. } & \multicolumn{2}{|c|}{ PTV05 } & \multicolumn{2}{|c|}{ PTV20 } \\
\hline & & $\overline{\mathrm{D}_{90}}$ & $\sigma_{\mathrm{D}_{90}}$ & $\overline{\mathrm{D}_{90}}$ & $\sigma_{\mathrm{D}_{90}}$ \\
\hline \multirow{2}{*}{${ }^{103} \mathrm{Pd}$} & $\mathrm{D}_{\mathrm{m}, \mathrm{m}(\mathrm{CT})}$ & 87.1 & 24.0 & 18.0 & 11.4 \\
\hline & TG- $43_{\text {sim }}$ & 93.2 & 22.0 & 18.7 & 2.6 \\
\hline \multirow[t]{2}{*}{${ }^{125} \mathrm{I}$} & $\mathrm{D}_{\mathrm{m}, \mathrm{m}(\mathrm{CT})}$ & 73.3 & 15.0 & 23.2 & 8.8 \\
\hline & TG-43 $43_{\text {sim }}$ & 91.6 & 18.0 & 26.8 & 3.0 \\
\hline \multirow{2}{*}{${ }^{131} \mathrm{Cs}$} & $\mathrm{D}_{\mathrm{m}, \mathrm{m}(\mathrm{CT})}$ & 68.6 & 12.3 & 25.0 & 7.8 \\
\hline & $\mathrm{TG}-43_{\operatorname{sim}}$ & 89.3 & 16.3 & 30.1 & 3.1 \\
\hline \multirow{2}{*}{$50 \mathrm{keV}$} & $\mathrm{D}_{\mathrm{m}, \mathrm{m}(\mathrm{CT})}$ & 68.9 & 8.7 & 32.3 & 3.8 \\
\hline & $\mathrm{TG}-43_{\mathrm{sim}}$ & 88.2 & 12.9 & 39.3 & 3.0 \\
\hline \multirow{2}{*}{$100 \mathrm{keV}$} & $\mathrm{D}_{\mathrm{m}}$ & 82.2 & 12.2 & 36.5 & 2.6 \\
\hline & $\mathrm{TG}-43_{\mathrm{sim}}$ & 88.5 & 12.9 & 40.1 & 2.9 \\
\hline
\end{tabular}

For PTV05, mean values of $\mathrm{D}_{90}$ decrease with increasing source energy for the three lowest energy sources $\left({ }^{103} \mathrm{Pd},{ }^{125} \mathrm{I}\right.$, and $\left.{ }^{131} \mathrm{Cs}\right)$ and both calculation methods 

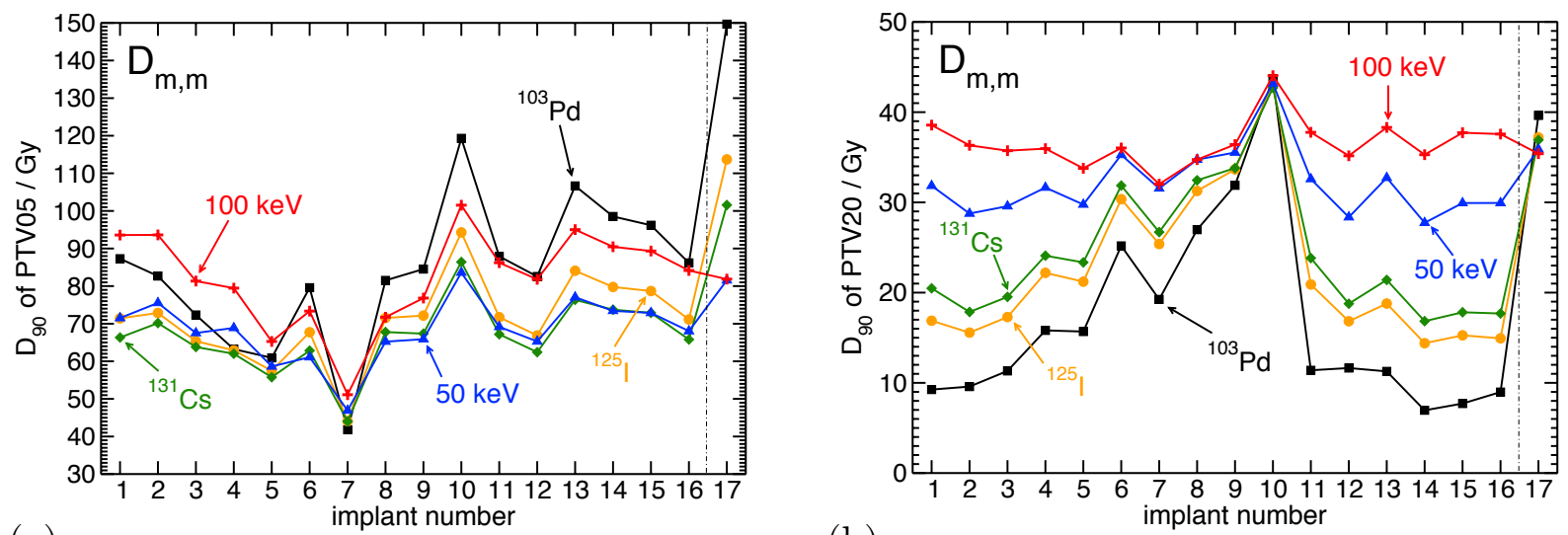

(a)
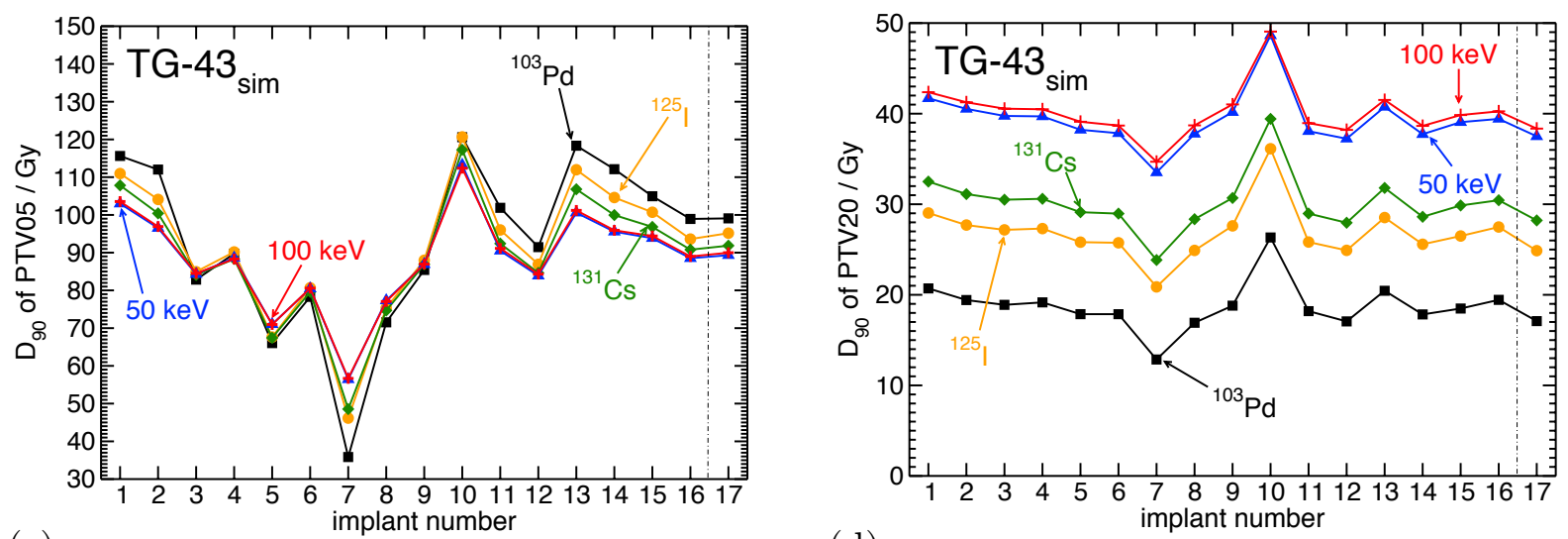

(c)

(d)

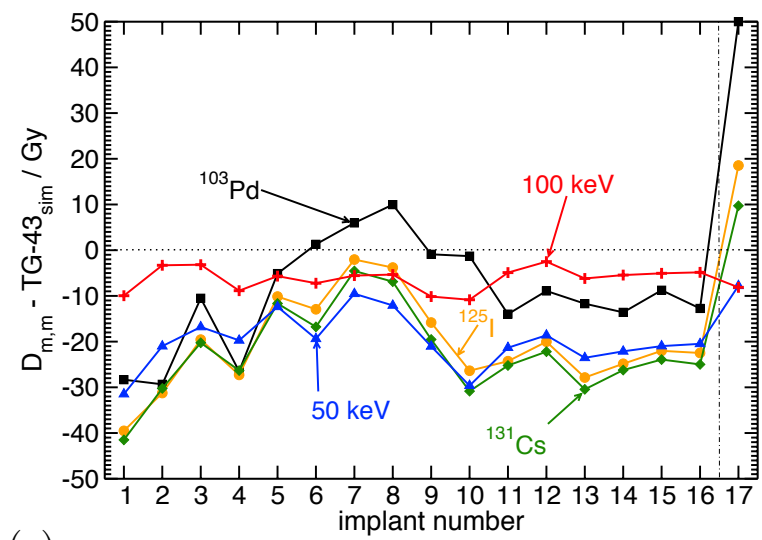

(e)

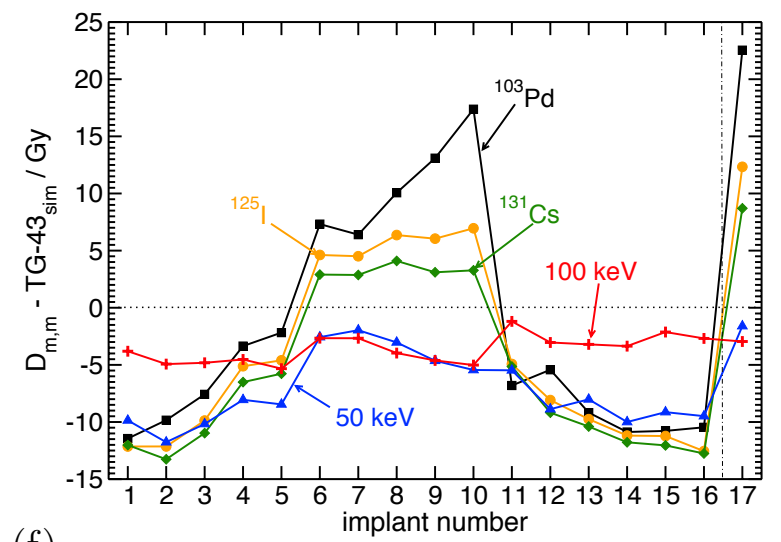

(f)

Figure 7.4: $\mathrm{D}_{90}$ of PTV05 (a and c) and PTV20 (b and d) for each source type and virtual implant for $\mathrm{D}_{\mathrm{m}, \mathrm{m}(\mathrm{CT})}$ and TG-43 $3_{\mathrm{sim}}$. The difference between $\mathrm{D}_{90}$ values for $\mathrm{D}_{\mathrm{m}, \mathrm{m}(\mathrm{CT})}$ and TG-43 4 sim for each source type and virtual implant for (e) PTV05 and (f) PTV20. Lines connecting doses for different virtual implants are provided to aid the eye. 
$\left(\mathrm{D}_{\mathrm{m}, \mathrm{m}(\mathrm{CT})}\right.$ and $\left.\mathrm{TG}-43_{\mathrm{sim}}\right)$. This reflects the fact that doses decrease more rapidly about lower-energy sources (compared to higher energy sources) and hence to achieve the same prescription dose (which is defined at $5 \mathrm{~mm}$ from the planar implants see section 7.1), doses near the sources are relatively higher for these lower-energy sources. For PTV20, values of $\mathrm{D}_{90}$ generally increase with increasing source energy due to the longer mean free path of photons for higher-energy sources (e.g., photon fluence will be greater near the edges of the PTV20 values for higher-energy sources) and the resulting more gradual dose fall-off with increasing distance from sources. Differences in $\mathrm{D}_{90}$ between TG-43 $3_{\text {sim }}$ and $\mathrm{D}_{\mathrm{m}, \mathrm{m}(\mathrm{CT})}$ calculations are due to the effects of non-water media which vary with source energy (table 7.1.

For both $\mathrm{D}_{\mathrm{m}, \mathrm{m}(\mathrm{CT})}$ and TG-43 $43_{\text {sim }}$ calculations, $\mathrm{D}_{90}$ varies to a greater degree for the implants along the medial side of the lung (implants 6-11) since these implants are the most deformed. However, $\mathrm{D}_{90}$ is generally more consistent across source types and across implants for TG-43 $3_{\text {sim }}$ than for $\mathrm{D}_{\mathrm{m}, \mathrm{m}(\mathrm{CT})}$ since TG- $43_{\text {sim }}$ calculations do not account for the effects of tissue heterogeneities and their variation with photon energy (see table 7.1). The effects of tissue heterogeneities also account for $\mathrm{D}_{90}$ generally being greater for $\mathrm{TG}-43_{\text {sim }}$ than for $\mathrm{D}_{\mathrm{m}, \mathrm{m}(\mathrm{CT})}$; however, this result is sensitive to the combination of source energy and tissues found in the vicinity of the implant. For example, $\mathrm{D}_{90}$ for the fissure implant (no. 17) for ${ }^{103} \mathrm{Pd}$ is much greater for $\mathrm{D}_{\mathrm{m}, \mathrm{m}(\mathrm{CT})}$ than for TG- $43_{\text {sim }}$ since this implant is surrounded solely by low density lung tissue which attenuates photons less and absorbs more dose than water at lower photon energies. The effects of tissue heterogeneities observed here are in agreement with the results presented in chapter 6 (in which the effects of lung brachytherapy tissue heterogeneities for ${ }^{125} \mathrm{I}$ and ${ }^{131} \mathrm{Cs}$ are discussed in greater detail).

Figure 7.5 shows $\mathrm{V}_{100}$ for PTV05 and PTV20 for the various implants and radionuclides with mean and standard deviations across the seventeen implants for 
$\mathrm{V}_{100}$ and $\mathrm{V}_{200}$ given in table 7.3 . Similar to $\mathrm{D}_{90}$, large variations in values of $\mathrm{V}_{100}$ are observed between source types and implants. In general, $\mathrm{V}_{100}$ is greatest for ${ }^{103} \mathrm{Pd}$ seeds as a result of a consistent prescription dose of 100 Gy being used for each source type: ${ }^{103} \mathrm{Pd}$ emits lower energy photons with shorter mean free paths than higher energy sources; consequently the dose to the prescription points for ${ }^{103} \mathrm{Pd}$ implants is largely produced by the contribution of photons from seeds closest to the prescription points. In contrast, higher energy sources can achieve the prescription dose by relying on the contribution from more sources since more emitted photons will reach the prescription points and beyond. This is confirmed by considering the mean and standard deviation of $\mathrm{V}_{200}$ across the seventeen implants for each source type: ${ }^{103} \mathrm{Pd}$ implants produce the greatest values of $\mathrm{V}_{200}$.

Table 7.3: The mean and standard deviations of $V_{100}$ and $V_{200}$ for the seventeen virtual implants for PTV05 and PTV20 (in percent of volume) for each source type and dose calculation method. See figure 7.5 for individual values of $V_{100}$.

\begin{tabular}{|c|c|c|c|c|c|c|c|c|c|}
\hline \multirow[b]{2}{*}{ Source } & \multirow[b]{2}{*}{ Dose calc. } & & \multicolumn{2}{|c|}{ PTV05 } & \multirow[b]{2}{*}{$\sigma_{\mathrm{V}_{200}}$} & \multirow[b]{2}{*}{$\overline{\mathrm{V}_{100}}$} & \multicolumn{2}{|c|}{ PTV20 } & \multirow[b]{2}{*}{$\sigma_{\mathrm{V}_{200}}$} \\
\hline & & $\overline{V_{100}}$ & $\sigma_{\mathrm{V}_{100}}$ & $\overline{V_{200}}$ & & & $\sigma_{\mathrm{V}_{100}}$ & $\overline{V_{200}}$ & \\
\hline \multirow{2}{*}{${ }^{103} \mathrm{Pd}$} & $\mathrm{D}_{\mathrm{m}, \mathrm{m}(\mathrm{CT})}$ & 83.2 & 9.2 & 41.1 & 11.1 & 43.4 & 4.4 & 14.0 & 2.2 \\
\hline & TG- $43_{\text {sim }}$ & 84.2 & 13.1 & 19.8 & 7.4 & 29.5 & 2.3 & 7.9 & 1.7 \\
\hline \multirow{2}{*}{${ }^{125} \mathrm{I}$} & $\mathrm{D}$ & 72.6 & 9.0 & 8.3 & 1.9 & 35.1 & 3.2 & 8.1 & 2.0 \\
\hline & TG & 83.6 & 12.0 & 11.6 & 6.8 & 29.1 & 2.6 & 5.0 & 1.5 \\
\hline \multirow{2}{*}{${ }^{131} \mathrm{Cs}$} & $\mathrm{D}_{\mathrm{m}, \mathrm{m}(\mathrm{CT})}$ & 67.8 & 9.6 & 12.9 & 5.5 & 31.3 & 3.2 & 7.5 & 2.5 \\
\hline & TG-4 & 82.1 & 11.7 & 9.0 & 6.2 & 28.3 & 2.7 & 4.3 & 1.3 \\
\hline \multirow{2}{*}{$50 \mathrm{keV}$} & & 59.5 & 9.5 & 8.6 & 4.9 & 25.1 & 4.6 & 7.5 & 4.4 \\
\hline & TG & 80.5 & 10.8 & 4.8 & 4.1 & 27.6 & 2.9 & 2.7 & 1.0 \\
\hline \multirow{2}{*}{$100 \mathrm{keV}$} & & 72. & $12.3^{\circ}$ & 8.3 & 4. & 26 & 4.7 & 4.2 & 1.3 \\
\hline & & 80.4 & 11.1 & 5.2 & 4.3 & 27.5 & 2.8 & 2.9 & 1.0 \\
\hline
\end{tabular}

Values of $\mathrm{V}_{100}$ are more consistent across source types for TG- $43_{\text {sim }}$ than for $\mathrm{D}_{\mathrm{m}, \mathrm{m}(\mathrm{CT})}$. This is due to the effects of media heterogeneities in $\mathrm{D}_{\mathrm{m}, \mathrm{m}(\mathrm{CT})}$ calculations which vary with source energy; further, dose is prescribed assuming a water 

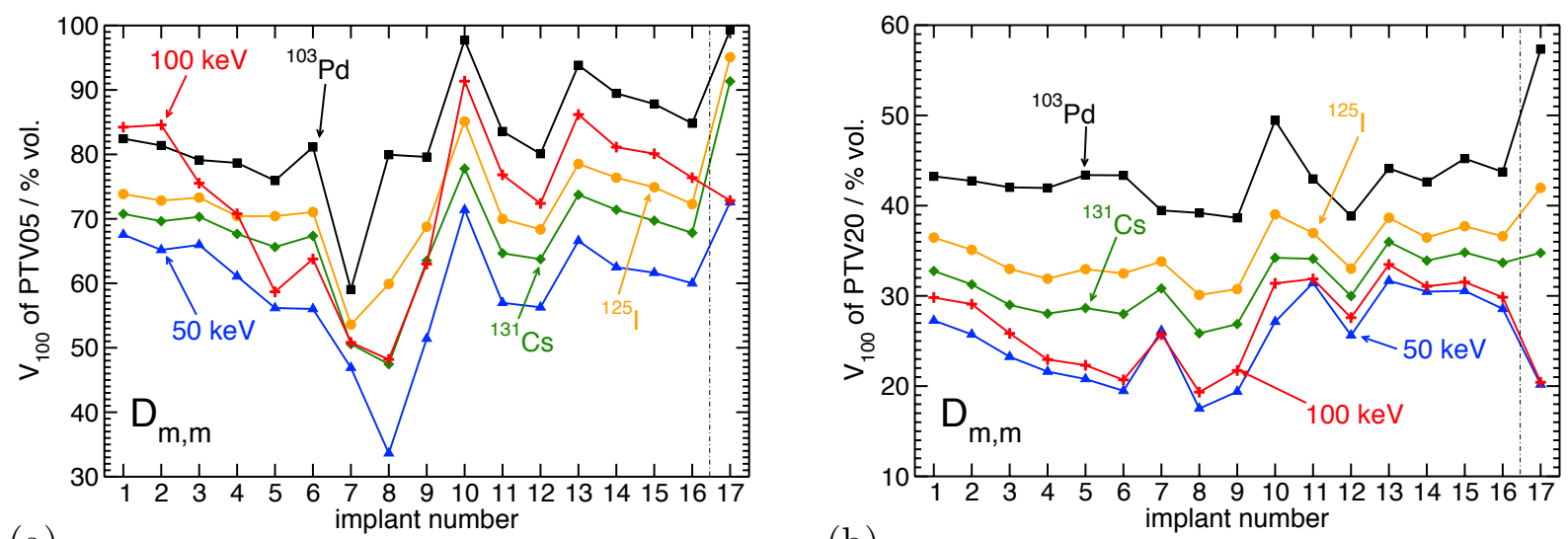

(a)
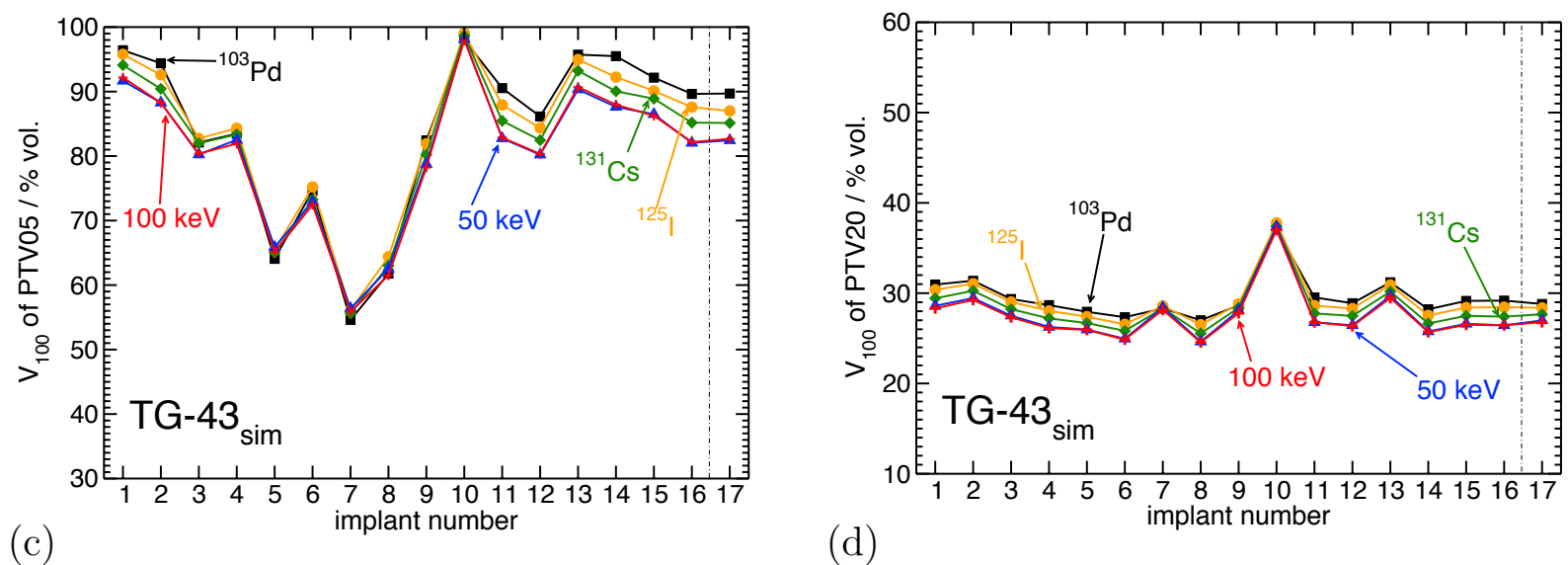

(d)

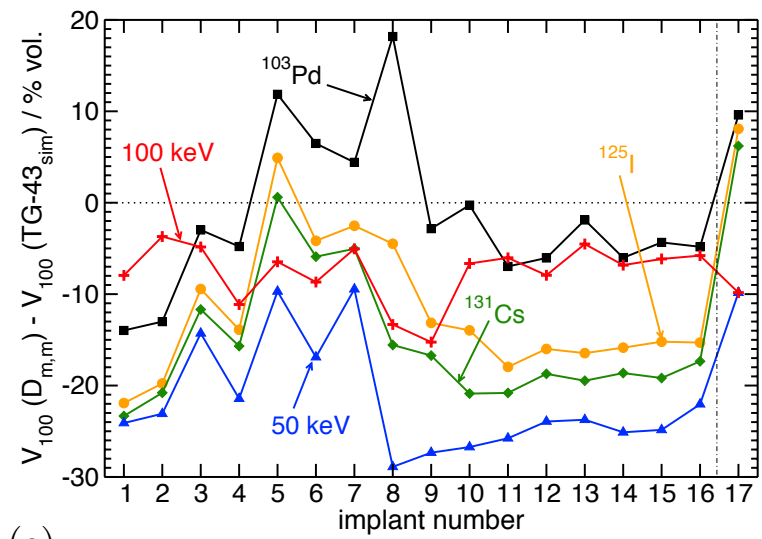

(e)

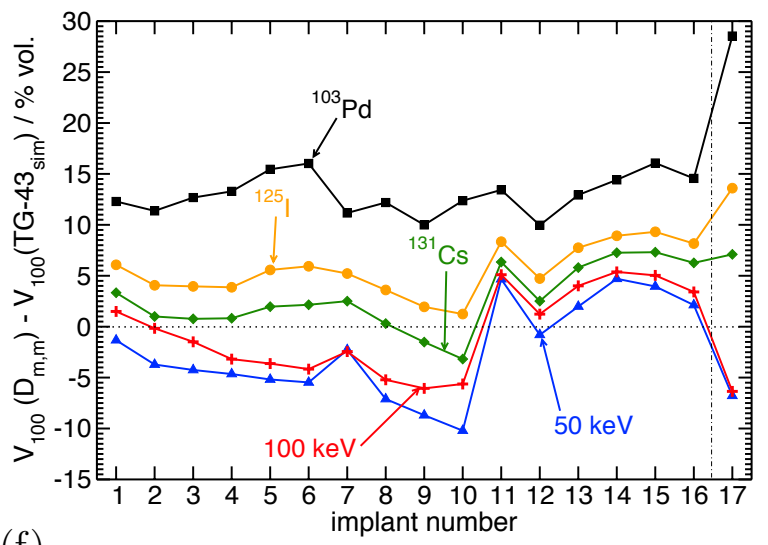

Figure 7.5: $\mathrm{V}_{100}$ of (a and c) PTV05 and (b and d) PTV20 for each source type and virtual implant for $\mathrm{D}_{\mathrm{m}, \mathrm{m}(\mathrm{CT})}$ and TG-43sim. The difference between $\mathrm{V}_{100}$ values for $\mathrm{D}_{\mathrm{m}, \mathrm{m}(\mathrm{CT})}$ and TG-43 $3_{\text {sim }}$ for each source type and virtual implant for (e) PTV05 and (f) PTV20. Lines connecting doses for different virtual implants are provided to aid the eye. 
environment (and ignoring the effects of non-water media). The effects of tissue heterogeneities generally result in larger values of $\mathrm{V}_{100}$ for $\mathrm{TG}-43_{\text {sim }}$ compared to $\mathrm{D}_{\mathrm{m}, \mathrm{m}(\mathrm{CT})}$ for PTV05; for PTV20, values of $\mathrm{V}_{100}$ for TG- $43_{\text {sim }}$ are generally less than or similar to those for $\mathrm{D}_{\mathrm{m}, \mathrm{m}(\mathrm{CT})}$.

For ${ }^{103} \mathrm{Pd},{ }^{125} \mathrm{I}$, and ${ }^{131} \mathrm{Cs}$ sources, $\mathrm{D}_{\mathrm{m}, \mathrm{m}(\mathrm{CT})}$ values of $\mathrm{V}_{100}$ are greater for implant 17 compared to other implants since implant 17 is surrounded solely by lung. The reduced attenuation in lung and increased energy absorption in lung tissue compared to water for lower energy photons (table 7.1) result in these relatively large values of $\mathrm{V}_{100}$ for $\mathrm{D}_{\mathrm{m}, \mathrm{m}(\mathrm{CT})}$ compared to TG-43 $3_{\text {sim }}$. Mass attenuation and absorption coefficient ratios of water to lung are closer to unity for $50 \mathrm{keV}$ and $100 \mathrm{keV}$ photons (table 7.1) and so these sources have lower values of $\mathrm{V}_{100}$ for $\mathrm{D}_{\mathrm{m}, \mathrm{m}(\mathrm{CT})}$ compared to TG- $43_{\text {sim }}$ due to a reduced contribution of dose from back-scattered photons.

Actual patient implants will differ from the virtual implants considered in this study due to varying resection sites and source implantation methods. However, the results presented in this section demonstrate that treatment volume dose metrics vary considerably with implant site and source type for both $\mathrm{D}_{\mathrm{m}, \mathrm{m}(\mathrm{CT})}$ and TG- $43_{\text {sim }}$ calculations. These variations are due to the combined effects of implant deformation, source energy, and tissues near the implant site $\left(\mathrm{D}_{\mathrm{m}, \mathrm{m}(\mathrm{CT})}\right.$ only); studying each effect in isolation dose not enable prediction of dose metrics for arbitrary treatment sites.

\subsubsection{Doses to the right lung}

Figure 7.6 shows the mean dose to the right lung for the seventeen implants and five sources for $\mathrm{D}_{\mathrm{m}, \mathrm{m}(\mathrm{CT})}$ and TG-43 $3_{\mathrm{sim}}$. Mean lung doses range between a maximum value of $27 \mathrm{~Gy}$ for ${ }^{103} \mathrm{Pd}$ and $\mathrm{D}_{\mathrm{m}, \mathrm{m}(\mathrm{CT})}$ for implant 17 and a minimum of 6 Gy for ${ }^{103} \mathrm{Pd}$ and TG- $43_{\text {sim }}$ for implant 6 . Differences between $D_{m, m(C T)}$ and $T G-43_{\text {sim }}$ values of mean 


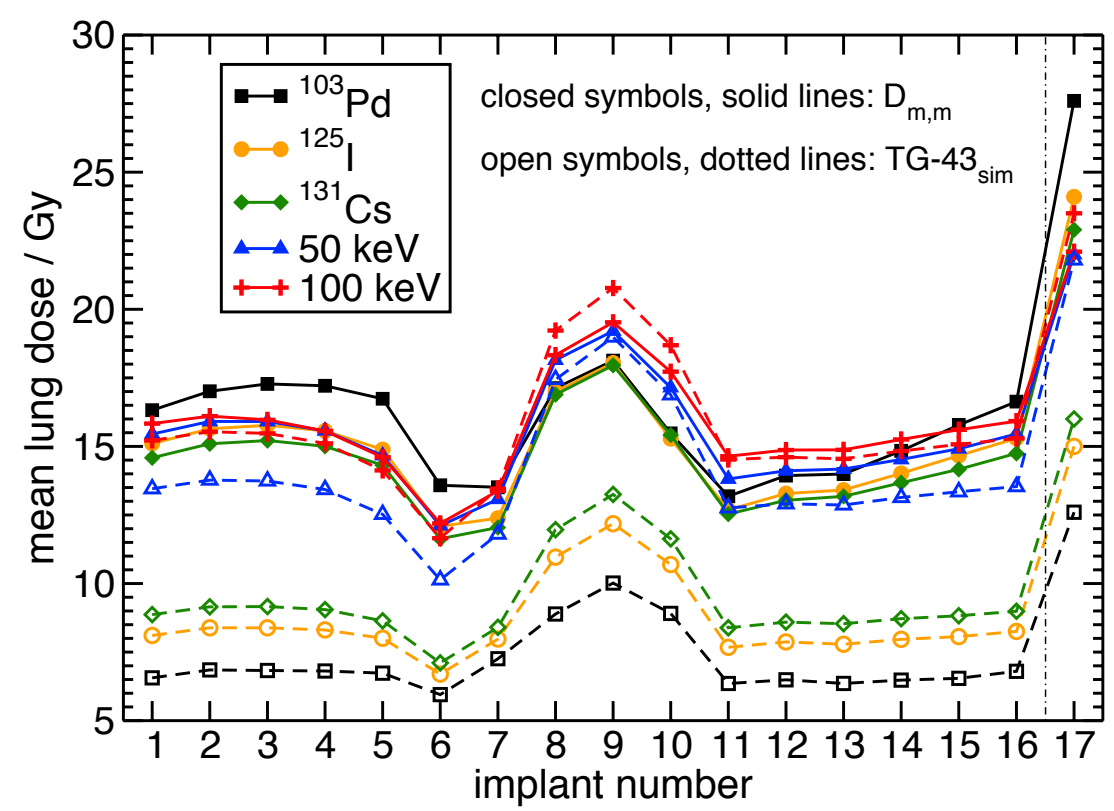

(a)

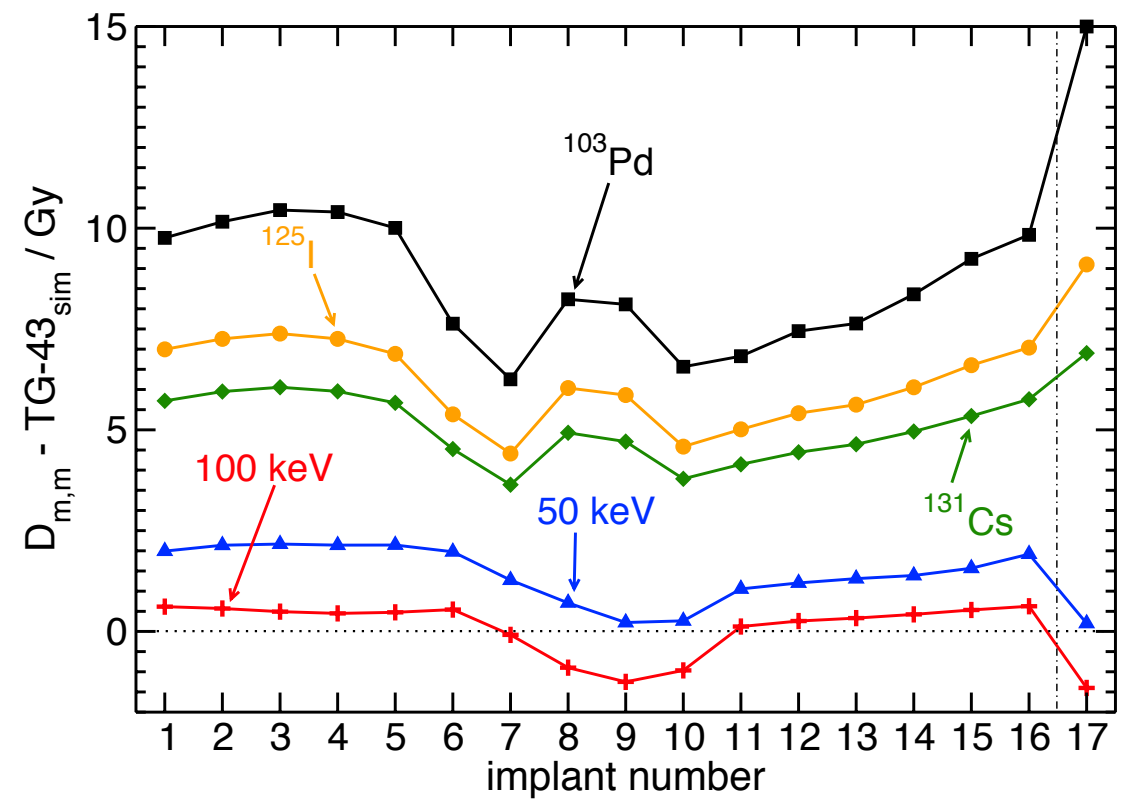

(b)

Figure 7.6: (a) Mean dose to the right (ipsilateral) lung for each source type and virtual implant for $\mathrm{D}_{\mathrm{m}, \mathrm{m}(\mathrm{CT})}$ and TG-43 $3_{\text {sim. }}$. (b) The difference between $\mathrm{D}_{\mathrm{m}, \mathrm{m}(\mathrm{CT})}$ and $\mathrm{TG}-43_{\text {sim }}$ for each source type and virtual implant. Lines connecting doses for different virtual implants are provided to aid the eye.

lung dose increase with decreasing photon energy: differences are greatest at 7 to 15 Gy for ${ }^{103} \mathrm{Pd}$ and smallest at -3 to 1 Gy for $100 \mathrm{keV}$ sources. For all source types, 
dose to the lung generally varies with distance of the implant from the centre of the lung: of the sixteen implants on the surface of the lung, implant 6 is the furthest from the centre of the lung (see figure 7.2 , page 122 and has the lowest mean doses while implant 9 is the closest to the centre and has the greatest. The fissure implant (no. 17) has considerably greater mean lung doses compared to the other implants since it is approximately in the centre of the lung.

The differences between $\mathrm{D}_{\mathrm{m}, \mathrm{m}(\mathrm{CT})}$ and TG-43 $3_{\text {sim }}$ for the mean lung dose of each implant (fig. 7.6p) consistently increase with decreasing energy and this can be explained by studying the dose distributions for the fissure implant (no. 17) in more detail. Figure 7.7 shows $\mathrm{D}_{\mathrm{m}, \mathrm{m}(\mathrm{CT})}$ and $\mathrm{TG}-43_{\text {sim }}$ dose volume histograms for the right lung volume calculated using the five sources and the fissure implant (no. 17). For TG- $43_{\text {sim }}$, the dose to the lung increases considerably with increasing mean emitted photon energy of the sources since higher energy photons are more penetrating in the modelled water. The mean dose to the lung (see table 7.4 increases approximately by a factor of two between ${ }^{103} \mathrm{Pd}$ and $100 \mathrm{keV}$ sources for TG- $43_{\text {sim }}$.

Table 7.4: Mean dose to the right lung for the fissure implant (no. 17) for each source model for $\mathrm{D}_{\mathrm{m}, \mathrm{m}(\mathrm{CT})}$ and TG- $43_{\mathrm{sim}}$.

\begin{tabular}{ccc}
\hline \hline \multirow{2}{*}{ Source } & \multicolumn{2}{c}{ Mean dose $(\mathrm{Gy})$} \\
\cline { 2 - 3 } & $\mathrm{D}_{\mathrm{m}, \mathrm{m}(\mathrm{CT})}$ & TG-43 $3_{\text {sim }}$ \\
\hline${ }^{103} \mathrm{Pd}$ & 27.6 & 12.6 \\
${ }^{125} \mathrm{I}$ & 24.1 & 15.0 \\
${ }^{131} \mathrm{Cs}$ & 22.9 & 16.0 \\
$50 \mathrm{keV}$ & 22.0 & 21.8 \\
$100 \mathrm{keV}$ & 22.1 & 23.5 \\
\hline \hline
\end{tabular}

The difference in photon attenuation and energy absorption between nominaldensity water and lower-density lung generally results in greater $\mathrm{D}_{\mathrm{m}, \mathrm{m}(\mathrm{CT})} \mathrm{DVHs}$ compared to TG-43 sim. Photon fluence will be higher in lower-density lung compared to 


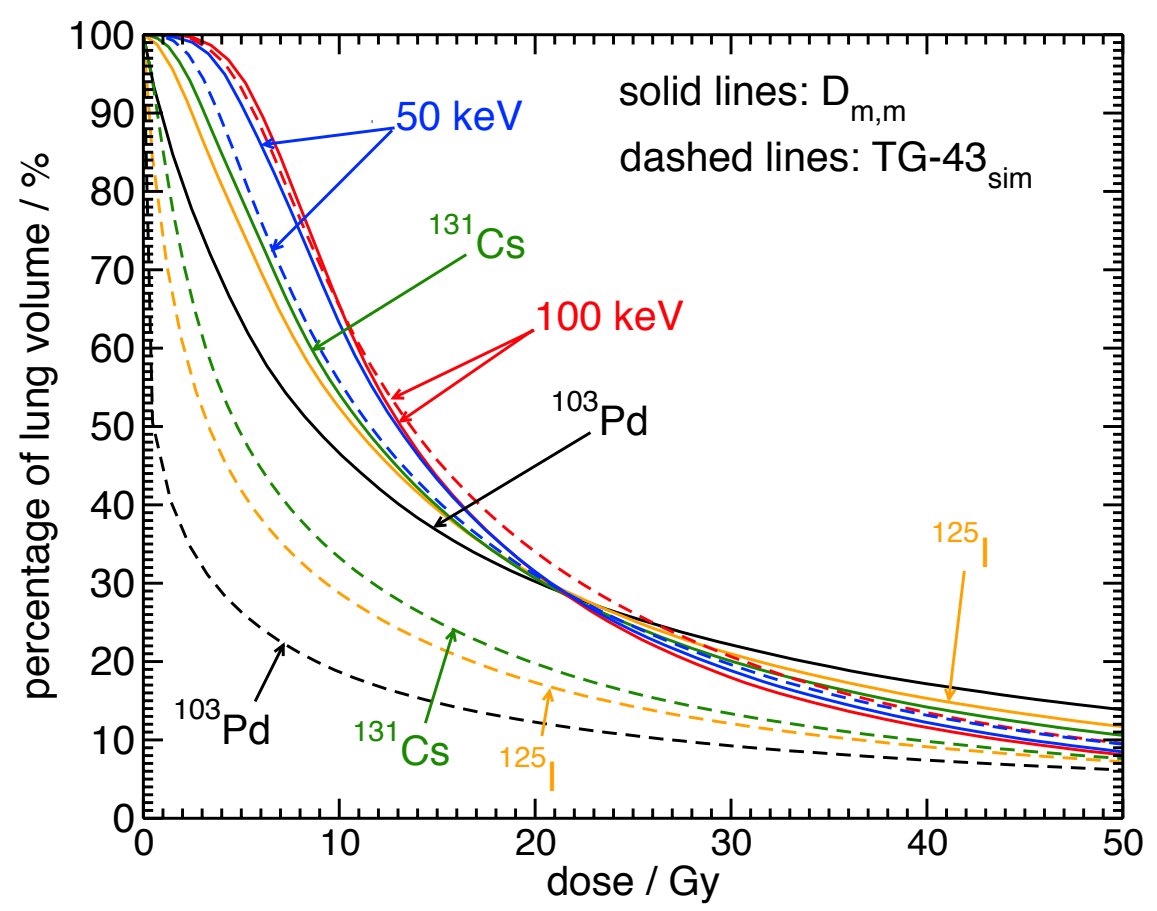

Figure 7.7: Dose volume histogram for the right lung for the fissure (no. 17) implant. DVHs for each radionuclide for $\mathrm{D}_{\mathrm{m}, \mathrm{m}(\mathrm{CT})}$ and $\mathrm{TG}-43_{\text {sim }}$ are shown.

in water and this greater fluence combined with the greater mass energy absorption coefficients of lung compared to water (table 7.1) result in higher $\mathrm{D}_{\mathrm{m}, \mathrm{m}(\mathrm{CT})}$ than TG- $43_{\text {sim }}$ for the lower-energy sources. As source energy increases, photon range increases and the influence that scattered photons have on mean dose to the lung becomes more significant. Thus, as source energy increases, the effect of a lower photon fluence in water compared to lung becomes reduced due to increased photon range and is increasingly compensated by a greater proportion of photons being scattered and contributing dose to the lung volume for TG- $43_{\text {sim }}$ compared to $\mathrm{D}_{\mathrm{m}, \mathrm{m}(\mathrm{CT})}$ where photons may escape the lung and contribute no dose. For many implants (including implant 17) the combined effects of differences in photon fluence and scattering conditions causes the trend in mean lung dose with source energy to reverse: for TG- $43_{\text {sim }}$ mean lung dose increases with source energy while it decreases with source energy for $\mathrm{D}_{\mathrm{m}, \mathrm{m}(\mathrm{CT})}$. 
Marks et al.118 suggest that for external beam radiotherapy treatments of the lung with conventional fractionation, $\mathrm{V}_{20}$ should be kept below $30-35 \%$ and that the mean lung dose be kept below 20-23 Gy to limit the risk of symptomatic radiation pneumonitis to less than or equal to $20 \%$. These guidelines may not be appropriate for brachytherapy due to radiobiological considerations (such as the effect of differences in dose rate and treatment length between brachytherapy and external beam radiotherapy). Additionally, prescription doses for radionuclides other than ${ }^{125}$ I will likely differ from the arbitrary 100 Gy used in this study. However, it is important to note that, for the prescription dose used in this work, TG-43 calculations indicate that lower energy sources produce lung doses well below the thresholds suggested by Marks et al. ${ }^{118}$, while the doses determined by model-based, $\mathrm{D}_{\mathrm{m}, \mathrm{m}(\mathrm{CT})}$ calculations are approximately equal to or greater than the thresholds. This underlines the importance of model-based dose calculations for comparing different radionuclides in low-energy brachytherapy.

\subsubsection{Doses to the aorta}

Figure $7.8 \mathrm{a}$ shows the mean aorta dose for each source type and virtual implant for $\mathrm{D}_{\mathrm{m}, \mathrm{m}(\mathrm{CT})}$ and $\mathrm{TG}-43_{\text {sim }}$ with differences between $\mathrm{D}_{\mathrm{m}, \mathrm{m}(\mathrm{CT})}$ and TG-43 $3_{\text {sim }}$ shown in figure 7.8b. For $100 \mathrm{keV}$ sources, doses range between approximately $1.5-11$ Gy for TG- $43_{\text {sim }}$ and between approximately 1.8 - 8 Gy for $D_{m, m(C T)}$ while doses for both TG- $43_{\text {sim }}$ and $\mathrm{D}_{\mathrm{m}, \mathrm{m}(\mathrm{CT})}$ stay below $0.5 \mathrm{~Gy}$ for ${ }^{103} \mathrm{Pd}$ seeds. Mean dose to the aorta (as a function of $\% \mathrm{D}_{\mathrm{Rx}}$ ) increases with increasing mean emitted photon energy and there is approximately an order of magnitude difference in mean aorta dose between ${ }^{103} \mathrm{Pd}$ and $100 \mathrm{keV}$ sources for implants near the aorta (e.g., implant 10) since the higher energy photons for 50 and $100 \mathrm{keV}$ monoenergetic sources are more penetrating. For

implants on the lateral side of the lung, aorta doses are near zero for ${ }^{103} \mathrm{Pd},{ }^{125} \mathrm{I}$, and ${ }^{131} \mathrm{Cs}$; however, aorta doses are non-zero for $50 \mathrm{keV}$ and $100 \mathrm{keV}$ sources. 


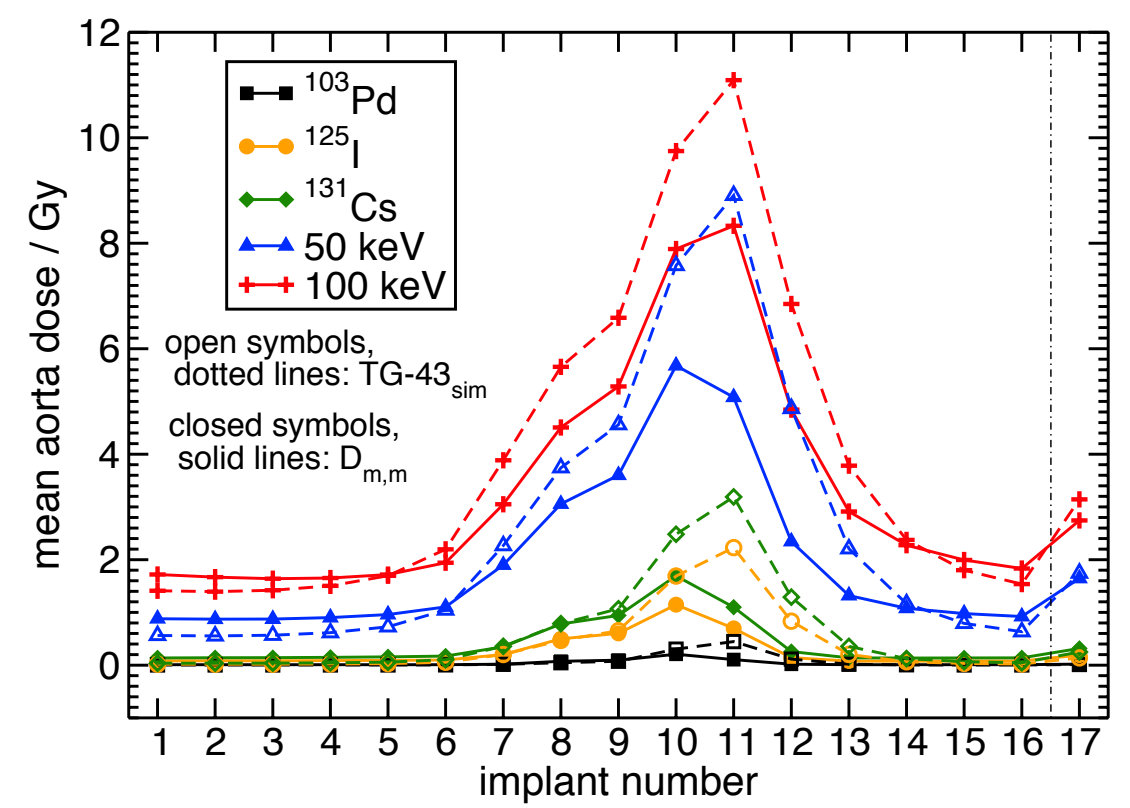

(a)

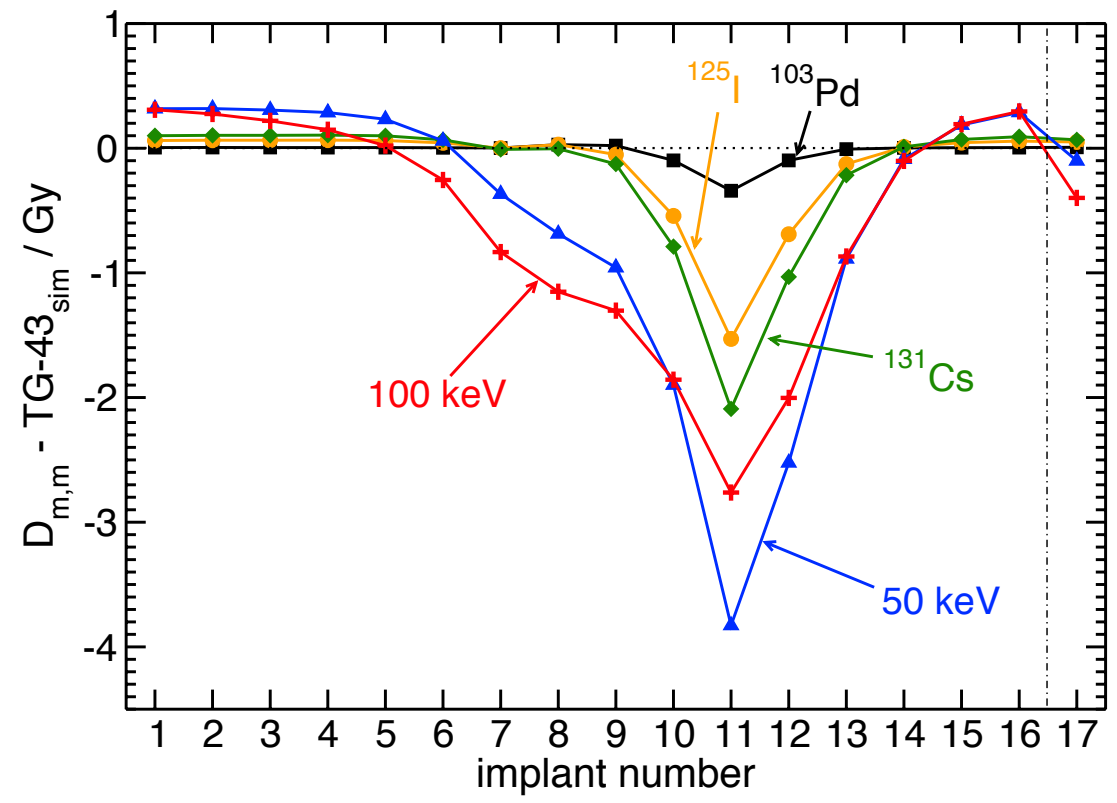

(b)

Figure 7.8: (a) The mean aorta dose for each source type and virtual implant for $\mathrm{D}_{\mathrm{m}, \mathrm{m}(\mathrm{CT})}$ and TG-43 $3_{\text {sim. }}$. (b) The difference between $\mathrm{D}_{\mathrm{m}, \mathrm{m}(\mathrm{CT})}$ and TG-43 $3_{\text {sim }}$ for each source type and virtual implant. . Lines connecting doses for different virtual implants are provided to aid the eye. 
The difference between mean aorta dose for $\mathrm{D}_{\mathrm{m}, \mathrm{m}(\mathrm{CT})}$ and TG-43 $3_{\text {sim }}$ is sensitive to implant position and photon energy spectra. For implants 7-13 where many photons travel through non-lung tissues to reach the aorta, the increased attenuation in these tissues compared to water results in larger values of TG- $43_{\operatorname{sim}}$ compared to $\mathrm{D}_{\mathrm{m}, \mathrm{m}(\mathrm{CT})}$. For implants on the lateral side of the lung (1-5 and 14-16) the majority of the dose to the aorta is produced by photons passing through the lung; the greater attenuation in water compared to lower-density lung results in TG- $43_{\text {sim }}$ being less than $\mathrm{D}_{\mathrm{m}, \mathrm{m}(\mathrm{CT})}$ for those source types for which doses are non-zero.

Weber et al. $\frac{119}{19}$ reported that the interstitial tolerance limit of rodent carotid arteries may exceed 13 Gy. In the context of permanent implant lung brachytherapy, Trombetta et al. ${ }^{117}$ suggested that the tolerance of the human aorta may exceed 12 Gy. Given this suggested tolerance, the mean doses reported in figure 7.8 are not likely to be of clinical concern. However, it is important to note that higher energy sources generally produce greater doses than lower energy sources and that TG-43 calculations generally overestimate the dose in cases where the dose is more significant. Additionally, a subset of calculations for implants modelled in the left lung show considerably higher doses, e.g., ${ }^{125} \mathrm{I}$ implants with centres at positions $a$ and $b$ (see figure 7.2 produce a mean aorta $\mathrm{D}_{\mathrm{m}, \mathrm{m}(\mathrm{CT})}$ of 90.2 and 15.5 Gy (89.9 and 13.1 Gy for TG-43 $3_{\text {sim }}$ ), respectively. Consequently, lung brachytherapy implants placed in the immediate vicinity of the aorta may produce mean aorta doses in excess of tolerances. Given the considerably higher doses for 50 and $100 \mathrm{keV}$ sources in figure 7.8, above-tolerance doses are even more likely for brachytherapy sources with mean emitted photon energies greater than that of ${ }^{125} \mathrm{I}$. 


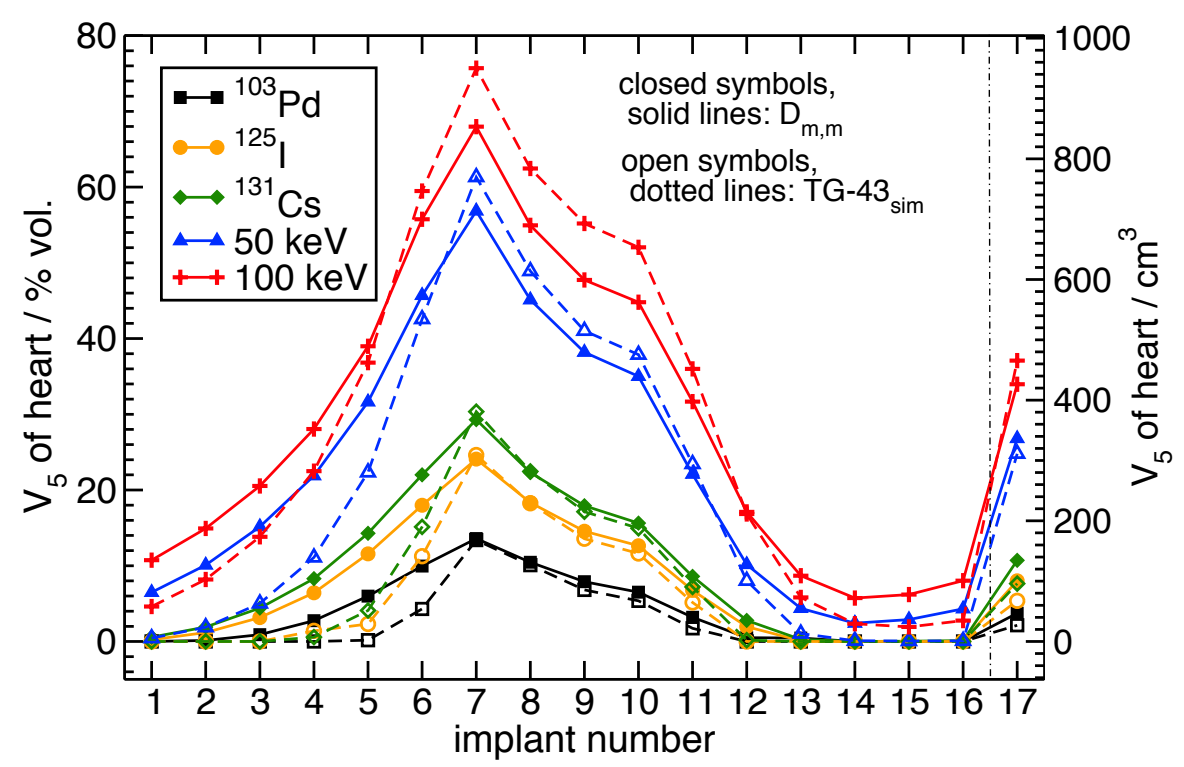

(a)

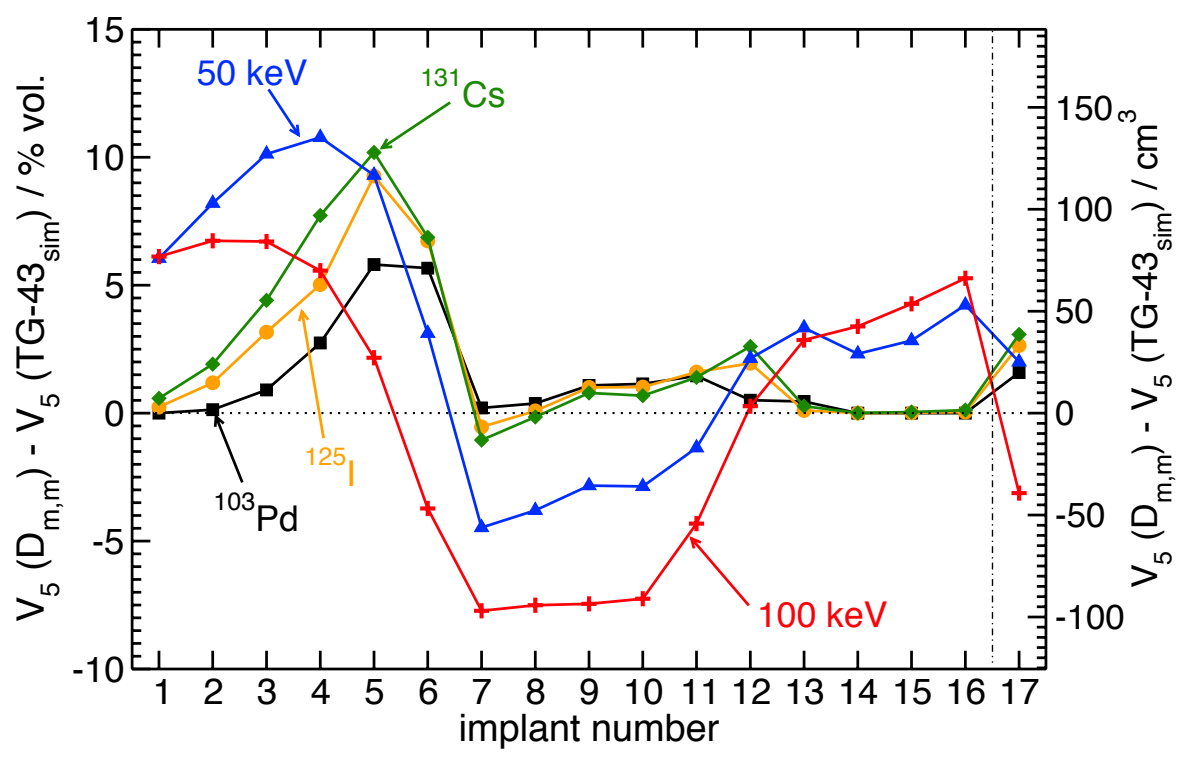

(b)

Figure 7.9: (a) The volume that receives at least 5 Gy $\left(\mathrm{V}_{5}\right)$ as a percent of the total heart volume and in $\mathrm{cm}^{3}$ for the heart for each source type and virtual implant for $\mathrm{D}_{\mathrm{m}, \mathrm{m}(\mathrm{CT})}$ and TG-43 $3_{\mathrm{sim}}\left(\mathrm{D}_{\mathrm{Rx}}=100 \mathrm{~Gy}\right)$. (b) The difference between $\mathrm{D}_{\mathrm{m}, \mathrm{m}(\mathrm{CT})}$ and $\mathrm{TG}-43_{\text {sim }}$ for each source type and virtual implant. Lines connecting doses for different virtual implants are provided to aid the eye. 


\subsubsection{Doses to the heart}

Figure 7.9 shows $V_{5}$ of the heart for the seventeen virtual implants and five source types for $\mathrm{D}_{\mathrm{m}, \mathrm{m}(\mathrm{CT})}$ and TG-43 $3_{\text {sim. }}$. Values of $\mathrm{V}_{5}$ are greatest for $100 \mathrm{keV}$ sources and lowest for ${ }^{103} \mathrm{Pd}$ with $\mathrm{V}_{5}$ values for $100 \mathrm{keV}$ sources ranging between approximately $10-70 \%$ for $\mathrm{D}_{\mathrm{m}, \mathrm{m}(\mathrm{CT})}$ and $5-75 \%$ for $\mathrm{TG}-43_{\mathrm{sim}}$ and volumes for ${ }^{103} \mathrm{Pd}$ ranging between $0-12 \%$ for both $\mathrm{D}_{\mathrm{m}, \mathrm{m}(\mathrm{CT})}$ and TG- $43_{\mathrm{sim}}$. For implants 7 and $8, \mathrm{~V}_{5}$ for $\mathrm{D}_{\mathrm{m}, \mathrm{m}(\mathrm{CT})}$ and TG-43 sim are in close agreement for ${ }^{103} \mathrm{Pd},{ }^{125} \mathrm{I}$, and ${ }^{131} \mathrm{Cs}$ implants while for 50 and $100 \mathrm{keV}$ implants, $\mathrm{V}_{5}$ is greater for TG-43 $3_{\text {sim }}$ than for $\mathrm{D}_{\mathrm{m}, \mathrm{m}(\mathrm{CT})}$ despite heart having a greater mass energy absorption coefficient than water at these energies. This is due to TG-43 calculations modelling more photons scattering in water for higherenergy sources and contributing more dose in the organ at risk than in model-based calculations where lower-density lung is modelled. For implants where photons must travel through lung tissue before reaching the heart (eg. implants 1-5 and 13-16), $\mathrm{V}_{5}$ is greater for $\mathrm{D}_{\mathrm{m}, \mathrm{m}(\mathrm{CT})}$ than for $\mathrm{TG}-43_{\text {sim }}$ as a result of reduced photon attenuation in lung than in water. $\mathrm{V}_{5}$ increases dramatically as source energy increases; $100 \mathrm{~cm}^{3}$ of the heart $(\approx 10 \%)$ receives 5 Gy for even the most distant $100 \mathrm{keV}$ source implants.

Increases in dose with source energy as well as differences between TG- $43_{\text {sim }}$ and $\mathrm{D}_{\mathrm{m}, \mathrm{m}(\mathrm{CT})}$ are also illustrated in figure 7.10 which shows the mean dose to the heart for the seventeen virtual implants and five source types. While mean $\mathrm{D}_{\mathrm{m}, \mathrm{m}(\mathrm{CT})}$ for the heart is greater for all implants and source types compared to mean $\mathrm{D}_{\mathrm{m}, \mathrm{m}(\mathrm{CT})}$ for the aorta, differences between TG- $43_{\text {sim }}$ and $\mathrm{D}_{\mathrm{m}, \mathrm{m}(\mathrm{CT})}$ for the mean heart dose are smaller than those shown for the aorta (figure 7.8).

Several studies (Darby et al. ${ }^{[120}$ and references therein) have suggested that the risk of death from heart disease has a linear relationship with mean dose to the heart with a recent study reporting an increase in major coronary events of $7.4 \%$ per Gy 121 . 


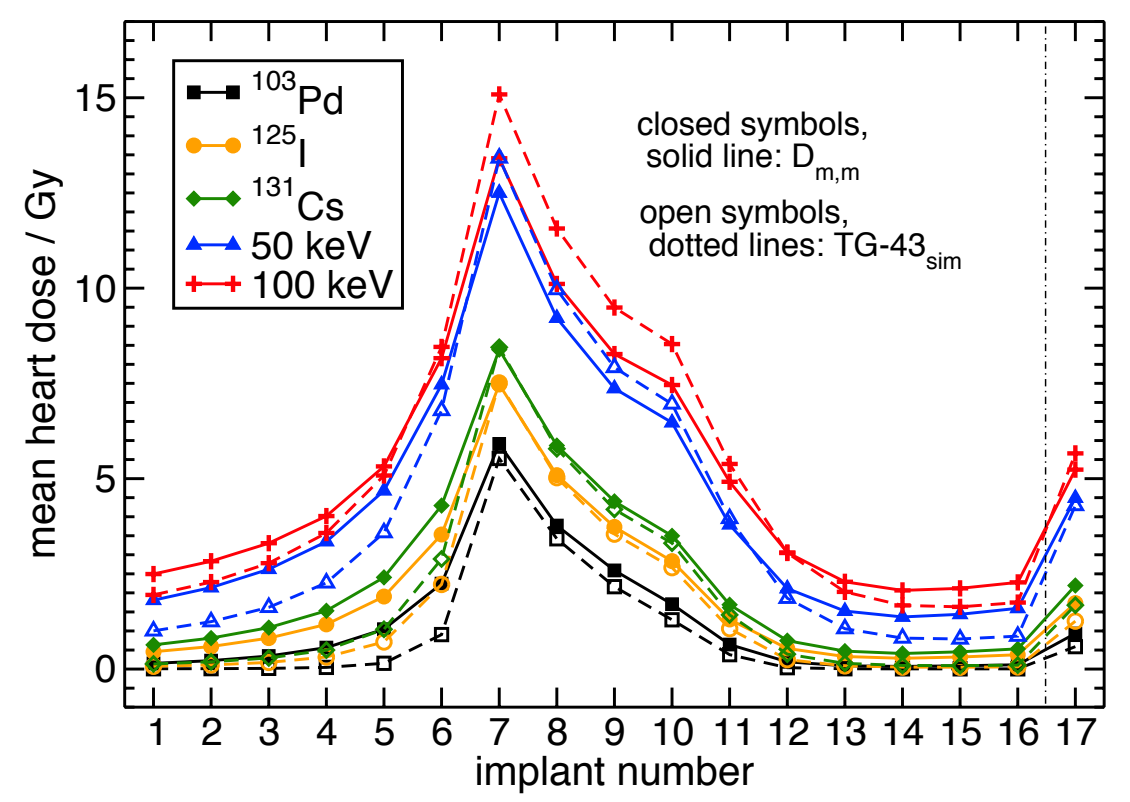

(a)

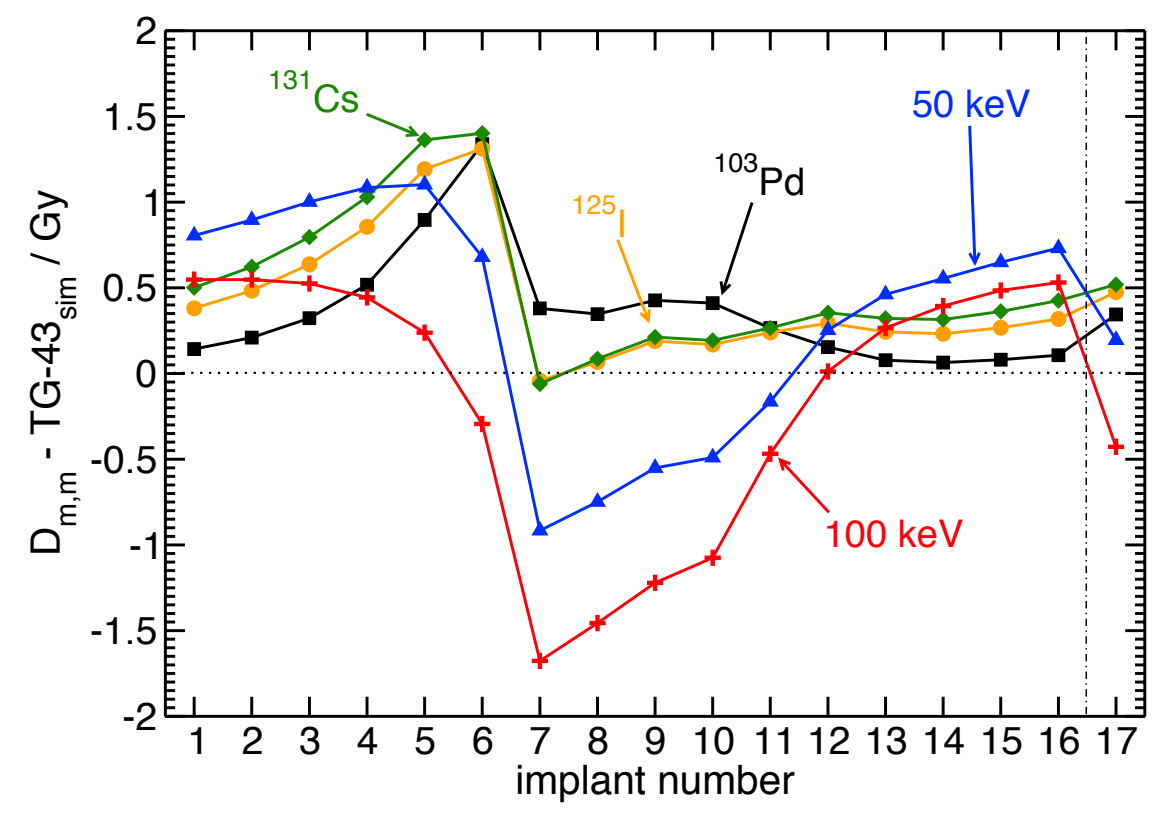

(b)

Figure 7.10: (a) The mean heart dose for each source type and virtual implant for $\mathrm{D}_{\mathrm{m}, \mathrm{m}(\mathrm{CT})}$ and TG-43 $3_{\text {sim. }}$. (b) The difference between $\mathrm{D}_{\mathrm{m}, \mathrm{m}(\mathrm{CT})}$ and TG- $43_{\text {sim }}$ for each source type and virtual implant. . Lines connecting doses for different virtual implants are provided to aid the eye. 
Given this apparent relationship of heart disease to dose, the modest over-and underestimations in mean dose due to TG-43 calculations as well as the greater mean heart doses for higher energy sources presented here should be taken into consideration.

\subsection{Conclusions}

Monte Carlo calculated doses to treatment volumes as well as to the ipsilateral lung, heart, and aorta were calculated for a variety of virtual implants within a computational phantom derived from patient CT and organ-contour data. Doses were calculated for ${ }^{103} \mathrm{Pd},{ }^{125} \mathrm{I}$, and ${ }^{131} \mathrm{Cs}$ seeds in addition to $50 \mathrm{keV}$ and $100 \mathrm{keV}$ monoenergetic point sources with full model-based calculations and TG-43-based calculations. Considerable variation with source energy and differences between model-based and TG-43-based doses were found for both treatment volumes and critical organs.

Differences in dose metrics to treatment volumes are due to the combined effects of source energy, implant deformation, and the attenuation, absorption, and scattering conditions due to the tissues in the vicinity of the implants (as discussed for ${ }^{125} \mathrm{I}$ and ${ }^{131} \mathrm{Cs}$ implants in chapter 6). Dose metrics for model-based calculations vary to a greater degree between source types and between implants than for TG-43-based calculations as model-based calculations account for tissue heterogeneity effects. The minimum doses received by the $90 \%$ hottest volumes $\left(\mathrm{D}_{90}\right)$ are generally greater for TG-43-based calculations than for model-based dose calculations but this is not true universally. In contrast with the results reported by Yang and Rivard ${ }^{55}$ (who found that mean PTV doses differed by only a few percent), treatment volume dose metrics were found to differ considerably (up to 50\%) between model-based and TG-43-based calculations. Dose metrics vary with source energy but the effect of source energy depends on the particular metrics and treatment volumes considered. 
For model-based calculations, mean doses to the ipsilateral lung decrease with increasing source energy while for TG-43-based calculations, mean doses to the lung increase considerably with increasing source energy. Differences between model-based and TG-43-based calculated mean dose to the lung increase for lower energy sources, underlining the importance of model-based dose calculations for comparing radionuclides in brachytherapy.

Doses to the heart and aorta generally increase with increasing source energy. For $100 \mathrm{keV}$ sources, clinically-relevant doses to the heart are calculated for even those implants on the lateral side of the lung and these doses are underestimated by approximately $50 \%$ by TG-43-based calculations. This has implications for the adoption of ${ }^{169} \mathrm{Yb}$ treatments with its mean emitted photon energy of $93 \mathrm{keV}$. TG43-based calculations generally underestimate the dose to the heart and aorta, in agreement with the results of Yang and Rivard 55 . However, for the implants nearest to the heart and aorta, TG-43-based calculations can overestimate the dose to these organs due to differences in attenuation and scattering conditions between water and patient-based phantoms.

The considerable differences in doses to treatment volumes and organs at risk between model-based and TG-43-based calculations as well as the strong variation in dose with source energy presented in this chapter suggest that the adoption of new radionuclides for brachytherapy should be undertaken with caution. The results of this chapter suggest that model-based dose calculations are crucial for the accurate study of radiobiological effects, selection of prescription doses, and comparison of clinical endpoints for permanent implant lung brachytherapy. 


\section{Chapter 8}

\section{Conclusions}

\subsection{Overview}

Compared to external beam radiation therapy, the use of patient-specific Monte Carlo calculations has only recently been utilized for brachytherapy. The predominant photon interactions for the energies of low-energy permanent implant brachytherapy cause significant differences between model-based dose calculations and the current water-based TG-43 approach. While considerable research had been done on this subject when the work for this thesis was initiated, many issues were still unresolved and, in the case of permanent implant brachytherapy, completely unexplored. This thesis studied the accurate application of Monte Carlo dose calculations to permanent implant breast and lung brachytherapy.

Current model-based dose calculation practices for permanent implant breast brachytherapy have previously involved the modelling of patient breasts with a single tissue with an elemental composition that averaged adipose and fibroglandular tissues. The changes in Monte Carlo calculated doses that occur with the segmentation of breast tissues in computational phantoms were investigated. Despite significant clinical adoption of permanent implant ${ }^{125} \mathrm{I}$ lung brachytherapy treatments and the

highly heterogeneous nature of the lung, doses for ${ }^{125}$ I lung brachytherapy have been 
calculated nearly exclusively with the water-based TG-43 formalism and no patientspecific model-based dose calculations had ever been reported before this work. In this thesis, the use of model-based dose calculations to determine patient-specific dose distributions for permanent implant lung brachytherapy patients was explored: the modelling choices for creating CT-derived patient-specific computational phantoms that significantly affect dose distributions were discovered and differences between model-based calculations and water-based TG-43 calculations were determined. Improved techniques for generating lung brachytherapy computational phantoms were studied by investigating various metallic artifact mitigation techniques and the use of organ-constrained tissue assignment schemes. Finally, Monte Carlo calculations were used to better understand the limitations of current treatment planning practices for permanent implant lung brachytherapy and to gain a comprehensive understanding of doses to treatment volumes and organs at risk and the variation of those doses with source energy.

\subsection{Summary of conclusions}

Chapter 3 presented a study of the segmentation of breast tissues in computational phantoms and found that the current practices at the time of using averaged tissue phantoms were insufficient for accurately simulating photon energy fluence for permanent implant breast brachytherapy. However, averaged-tissues were found to be sufficient for calculating dose volume metrics provided that the metrics were reported for gland and adipose tissues combined and that no calcifications were present. The methods commonly used in mammography radiation protection calculations were found to overestimate the average dose to glandular tissue.

Chapter 4 presented an initial exploration of model-based dose calculations for 
${ }^{125}$ I lung brachytherapy. Patient specific Monte Carlo dose calculations for permanent implant lung brachytherapy differed significantly from water-based TG-43 calculations and were sensitive to metallic artifact reduction techniques and tissue assignment schemes. Doses were found to be insensitive to small errors in the assignment of voxel mass density and to the padding of computational phantoms with regions of water.

Improvements on the patient-specific Monte Carlo calculations of chapter 4 were presented in chapter 5 which investigated the use of various metallic artifact mitigation techniques and organ-constrained tissue assignment for lung brachytherapy. Of the phantom models studied, the most accurate model was determined to be a computational phantom generated using a tissue assignment scheme that constrained assignment within lung contours to only lung and air that was applied to CT images that received virtual sinogram metallic artifact reduction followed by simple threshold replacement. Improved dose distributions for phantoms generated using organ-constrained tissue assignment schemes served to reinforce the recommendations in the report of the AAPM Task Group 186 on model-based dose calculation methods in brachytherapy beyond the TG-43 formalism. Results suggested that the variation in the unknown composition of fibrotic tissue was not likely to significantly affect dose distributions.

Chapter 6 used Monte Carlo methods to investigate lung brachytherapy treatment planning. Calculated prescription doses were found to change significantly with the medium in which dose was calculated. Calculating a prescription dose to two points defined above a planar grid was found to be insufficient for reliably producing desired dose distributions in patients. The results of this chapter suggested that any effective treatment planning practice for permanent implant lung brachytherapy would need to account, at least approximately, for the deformation of implants in 
patients and the tissues found in the vicinity of the implants.

Chapter 7 presented Monte Carlo calculated doses to treatment volumes and organs at risk for permanent implant lung brachytherapy. Differences between modelbased and TG-43-based doses and the variation of doses with source energy were studied. Considerable differences between model-based and TG-43-based doses were observed for both treatment volumes and critical organs. Doses to critical organs and treatment volumes as well as differences between model-based and TG-43-based calculated doses varied significantly with source energy. TG-43-based calculations generally underestimated the dose to the heart and aorta except for implants nearest to the heart and aorta where TG-43-based calculations overestimated the dose to these organs due to differences in attenuation and scattering conditions between water- and patient-based phantoms. These results underline the importance of using model-based dose calculation algorithms to achieve accurate dose calculations of permanent lung implant brachytherapy and that MBDCAs must play a crucial role in future study of radiobiological effects, the selection of prescription doses, and in the comparison of clinical endpoints for these treatments.

\subsection{Impact and future work}

This thesis represents a contribution towards the accurate application of patient specific Monte Carlo calculations for permeant implant breast and lung brachytherapy and for low-energy brachytherapy in general. The use of tissue segmentation in brachytherapy, which had previously been largely ignored, was shown to significantly affect dose distributions. The comparison of metallic artifact methods in chapters 4 and 5 and the calculation of resulting dose distributions from artifact corrected phantoms as well as the comparison of unconstrained and organ-constrained tissue 
assignments for brachytherapy represent novel contributions to the field of modelbased dose calculations. Additionally, this work presented insights into the treatment planning, doses to treatment volumes and organs at risk, and the variation in doses with radionuclides for permanent implant lung brachytherapy which, to date, had been informed entirely by water-based TG-43 calculations. Despite these contributions, continuation of the work of this thesis as well as improvements in the application of model-based dose calculations to low-energy brachytherapy in general are required before these methods can be adopted clinically.

The model-based dose calculation techniques for lung brachytherapy patients described in chapters 4 and 5 would likely require experimental validation before clinical adoption. This could be achieved by creating a physical phantom of a realistic human chest containing a removable lung brachytherapy implant. By acquiring CT images of the phantom with and without brachytherapy seeds, the performance of the metallic artifact reduction methods could be tested by comparing dose distributions generated from explicitly defined and CT-derived phantom models. Additionally, given prior knowledge of the elemental composition of the phantom structures, tissue assignment schemes which convert CT number to elemental compositions could be validated.

The work presented in this thesis illustrated the strong sensitivity of dose distributions to voxel elemental compositions for low-energy brachytherapy. Confirmation of the elemental compositions of human tissues, which have been reported by only a few studies in the past 30 years, as well as a comprehensive study of the variation of tissue compositions between people is necessary to reach sufficient confidence in patient-specific Monte Carlo dose calculations of low-energy brachytherapy for clinical adoption. 


\section{References}

[1] Canadian Cancer Society, Canadian Cancer Statistics 2013, Technical report, Canadian Cancer Society, Toronto, Canada, 2013. (page 1)

[2] Canadian Cancer Society, Radiation Therapy: A guide for people with cancer 2013, Technical report, Canadian Cancer Society, Toronto, Canada, 2013. (page 1)

[3] National Cancer Institute, Radiation therapy for cancer, 2013. (page 1)

[4] E. B. Podgorsak, editor, Radiation Oncology Physics: A Handbook for Teachers and Students, Educational Report Series, IAEA, Vienna, Austria, 2005. (page2)

[5] B. S. Hilaris, U. K. Henschke, and J. G. Holt, Clinical experience with long half-life and low-energy encapsulated radioactive sources in cancer radiation therapy, Radiology 91, 1163-1167 (1968). (page 2)

[6] J.-P. Pignol, B. Keller, E. Rakovitch, R. Sankreacha, H. Easton, and W. Que, First report of a permanent breast ${ }^{103} \mathrm{Pd}$ seed implant as adjuvant radiation treatment for early-stage breast cancer, Int. J. Radiat. Oncol. Biol. Phys. 64, $176-181$ (2006). (pages 2, 3)

[7] B. Keller, R. Sankreacha, E. Rakovitch, P. O'Brien, and J.-P. Pignol, A permanent breast seed implant as partial breast radiation therapy for early-stage patients: A comparison of palladium-103 and iodine-125 isotopes based on radiation safety considerations, Int. J. Radiat. Oncol. Biol. Phys. 62, $358-365$ (2005). (page 2)

[8] T. A. d'Amato, M. Galloway, G. Szydlowski, A. Chen, and R. J. Landreneau, Intraoperative brachytherapy following thoracoscopic wedge resection of stage I lung cancer, Chest 114, 1112 - 1115 (1998). (pages 2, 4)

[9] A. Chen, M. Gallowy, R. Landreneau, T. d'Amato, A. Colonias, S. Karlovits, A. Quinn, T. Santucci, S. Kalnicki, and D. Brown, Intraoperative ${ }^{125} \mathrm{I}$ brachytherapy for high-risk stage I non-small cell lung carcinoma, Int. J. Radiat. Oncol. Biol. Phys. 44, 1057 - 1063 (1999). (pages 2, 4, 43, 95)

[10] T. Whelan, R. MacKenzie, J. Julian, M. Levine, W. Shelly, L. Grimard, B. Lada, H. Lukka, F. Perera, and A. F. et.al., Randomized trial of breast irradiation schedules after lumpectomy for women with lymph node-negative breast cancer, Journal of the National Cancer Institute 94, 1143-1150 (2002). (page 3) 
[11] Canadian Cancer Society, Canadian Cancer Statistics 2011, Technical report, Canadian Cancer Society, Toronto, Canada, 2011. (page 4)

[12] American Cancer Society, Cancer Facts and Figures, Technical report, American Cancer Society, 2011. (page 4)

[13] R. Rami-Porta and M. Tsuboi, Sublobar resection for lung cancer, European Respiratory Journal 33, 426 - 435 (2009). (page 4)

[14] W. H. Warren and L. P. Faber, Segmentectomy versus lobectomy in patients with stage I pulmonary carcinoma: five year survival and patterns of intrathoracic recurrence, J. Thorac. Cardiovasc. Surg. 107, 1087-1094 (1994). (page 4)

[15] M. Johnson, A. Colonias, D. Parda, M. Trombetta, O. Gayou, B. Reitz, and M. Miften, Dosimetric and technical aspects of intraoperative I-125 brachytherapy for stage I non-small cell lung cancer, Phys. Med. Biol. 52, 1237 - 1245 (2007). (pages 4, 95, 98, 123)

[16] W. Lee, B. D. T. Daly, T. A. DiPetrillo, D. M. Morelli, A. C. Neuschatz, J. Morr, and M. J. Rivard, Limited resection for non-small cell lung cancer: Observed local control with implantation of I-125 brachytherapy seeds, Ann. Thorac. Surg. 75, $237-243$ (2003). (page 4)

[17] R. Santos, A. Colonias, D. Parda, M. Trombetta, R. H. Maley, R. Macherey, S. Bartley, T. Santucci, R. J. Keenan, and R. J. Landreneau, Comparison between sublobar resection and ${ }^{125}$ Iodine brachytherapy after sublobar resection in high-risk patients with Stage I non-small-cell lung cancer, Surgery 134, 691 - 697 (2003). (pages 4,118 )

[18] G. Voynov, D. E. Heron, C. J. Lin, S. Burton, A. Chen, A. Quinn, R. Santos, A. Colonias, and R. J. Landreneau, Intraoperative ${ }^{125}$ I Vicryl mesh brachytherapy after sublobar resection for high-risk stage I nonsmall cell lung cancer, Brachytherapy 4, 278 - 285 (2005). (page 4)

[19] A. J. Stewart, S. Mutyala, C. L. Holloway, Y. L. Colson, and P. M. Devlin, Intraoperative seed placement for thoracic malignancy - A review of technique, indictations, and published literature, Brachytherapy 8, 63-69 (2009). (page 4)

[20] S. Mutyala, A. Steward, A. J. Khan, R. A. Cormack, D. O'Farrell, D. Sugarbaker, and P. M. Devlin, Permanent iodine-125 interstitial planar seed brachytherapy for close or positive margins for thoracic malignancies, Int. J. Radiat. Oncol. Biol. Phys. 76, 1114 - 1120 (2010). (page 4)

[21] B. Parashar, A. G. Wernicke, A. Pavese, P. Singh, S. Trichter, A. Sabbas, D. I. Kutler, W. Kuhel, J. L. Port, P. C. Lee, D. Nori, and K. S. C. Chao, Cesium-131 permanent seed brachytherapy: Dosimetric evaluation and radiation exposure to surgeons, radiation oncologists, and staff, Brachytherapy 10, $508-513$ (2011). (pages 4, 95, 117, 118) 
[22] K. L. Leonard and T. A. DiPetrillo and J. J. Munro and D. E. Wazer, A novel ytterbium-169 brachytherapy source and delivery system for use in conjunction with minimally invasive wedge resection of early-stage lung cancer, Brachytherapy 10, 163 - 169 (2011). (pages 4, 117)

[23] H. C. Fernando, R. J. Landreneau, S. J. Mandrekar, S. L. Hillman, F. C. Nichols, B. Meyers, T. A. DiPetrillo, D. Heron, D. R. Jones, and B. D. T. Daly, The impact of adjuvant brachytherapy with sublobar resection on pulmonary function and dyspnea in high-risk patients with operable disease: preliminary results from the American College of Surgeons Oncology Group Z4032 trial, The Journal of thoracic and cardiovascular surgery 142, 554-562 (2011). (page 4)

[24] M. J. Rivard, J. L. M. Venselaar, and L. Beaulieu, The evolution of brachytherapy treatment planning, Med. Phys. 36, 2136 - 2153 (2009). (pages 5, 7, 8, 43.

[25] R. Nath, L. L. Anderson, G. Luxton, K. A. Weaver, J. F. Williamson, and A. S. Meigooni, Dosimetry of interstitial brachytherapy sources: Recommendations of the AAPM Radiation Therapy Committee Task Group No. 43, Med. Phys. 22, 209 - 234 (1995). (pages 5, 43, 95)

[26] M. J. Rivard, B. M. Coursey, L. A. DeWerd, M. S. Huq, G. S. Ibbott, M. G. Mitch, R. Nath, and J. F. Williamson, Update of AAPM Task Group No. 43 Report: A revised AAPM protocol for brachytherapy dose calculations, Med. Phys. 31, 633 - 674 (2004). (pages 5, 6, 7, 19, 43, 95, 97)

[27] M. J. Rivard, W. M. Butler, L. A. DeWerd, M. S. Huq, G. S. Ibbott, A. S. Meigooni, C. S. Melhus, M. G. Mitch, R. Nath, and J. F. Williamson, Supplement to the 2004 update of the AAPM Task Group No. 43 Report, Med. Phys. 34, $2187-2205$ (2007). (pages 5, 43, 95, 99)

[28] N. Papanikolaou, J. J. Battista, A. L. Boyer, C. Kappas, E. Klein, T. R. Mackie, M. Sharpe, and J. van Dyk, Tissue inhomogeneity corrections for megavoltage photon beams, AAPM Report 85, Medical Physics Pub for AAPM, New York, 2004. (page 8)

[29] L. Beaulieu, A. C. Tedgren, J.-F. Carrier, S. D. Davis, F. Mourtada, M. J. Rivard, R. M. Thomson, F. Verhaegen, T. A. Wareing, and J. F. Williamson, Report of the Task Group 186 on model-based dose calculation methods in brachytherapy beyond the TG-43 formalism: Current status and recommendations for clinical implementation, Med. Phys. 39, 6208 - 6236 (2012). (pages 8 , 73. 94.

[30] M. J. Berger and J. H. Hubbell, XCOM: Photon cross sections on a personal computer, Report NBSIR87-3597, National Institute of Standards Technology (NIST), Gaithersburg, MD 20899, U.S.A., 1987. (pages 9, 16, 21, 51, 97, 120)

[31] P. Mobit and I. Badragan, Dose perturbation effects in prostate seed implant brachytherapy with I-125, Phys. Med. Biol. 49, 3171 - 3178 (2004). (page 9) 
[32] B. Reniers, F. Verhaegen, and S. Vynckier, The radial dose function of lowenergy brachytherapy seeds in different solid phantoms: comparison between calculations with the EGSnrc and MCNP4C Monte Carlo codes and measurements, Phys. Med. Biol. 49, 1569 - 1582 (2004). (page 9)

[33] O. Chibani, J. F. Williamson, and D. Todor, Dosimetric effects of seed anisotropy and interseed attenuation for ${ }^{103} \mathrm{Pd}$ and ${ }^{125} \mathrm{I}$ prostate implants, Med. Phys. 32, 2557 - 2566 (2005). (pages 9, 10)

[34] J.-F. Carrier, L. Beaulieu, F. Therriault-Proulx, and R. Roy, Impact of interseed attenuation and tissue composition for permanent prostate implants, Med. Phys. 33, 595 - 604 (2006). (pages 9, 10)

[35] H. Afsharpour, M. D’Amours, B. Coté, J.-F. Carrier, F. Verhaegen, and L. Beaulieu, A Monte Carlo study on the effect of seed design on the interseed attenuation in permanent prostate implants, Med. Phys. 35, $3671-3681$ (2008). (pages 9, 10)

[36] R. E. Taylor, Monte Carlo Calculations for Brachytherapy, MSc Thesis, Carleton University, Ottawa, Ontario (2006). (page 9)

[37] J.-F. Carrier, M. D’Amours, F. Verhaegen, B. Reniers, A.-G. Martin, E. Vigneault, and L. Beaulieu, Postimplant dosimetry using a Monte Carlo dose calculation engine: a new clinical standard, Int. J. Radiat. Oncol. Biol. Phys. 68, 1190 - 1198 (2007). (pages 9, 10)

[38] C. S. Melhus and M. J. Rivard, COMS eye plaque brachytherapy dosimetry simulations for ${ }^{103} \mathrm{Pd},{ }^{125} \mathrm{I}$, and ${ }^{131} \mathrm{Cs}$, Med. Phys. 35, $3364-3371$ (2008). (pages 9, 10)

[39] R. M. Thomson, R. E. P. Taylor, and D. W. O. Rogers, Monte Carlo dosimetry for ${ }^{125} \mathrm{I}$ and ${ }^{103} \mathrm{Pd}$ eye plaque brachytherapy, Med. Phys. 35, $5530-5543$ (2008). (pages 9, 10)

[40] C. Furstoss et al, Monte Carlo study of LDR seed dosimetry with an application in a clinical brachytherapy breast implant, Med. Phys. 36, 1848 - 1858 (2009). (pages 9, 10, 22)

[41] H. Afsharpour, J.-P. Pignol, B. Keller, J.-F. Carrier, B. Reniers, F. Verhaegen, and L. Beaulieu, Influence of breast composition and interseed attenuation in dose calculations for post-implant assessment of permanent breast $103 \mathrm{Pd}$ seed implant, Phys. Med. Biol. 55, 4547 - 4561 (2010). (pages 9, 10, 22)

[42] G. Landry, B. Reniers, L. Murrer, L. Lutgens, E. Bloemen-Van Gurp, J.-P. Pignol, B. Keller, L. Beaulieu, and F. Verhaegen, Sensitivity of low energy brachytherapy Monte Carlo dose calculations to uncertainties in human tissue composition., Med. Phys. 37, 5188 - 5198 (2010). (pages 9, 10, 102)

[43] S. H. Cho, O. N. Vassiliev, S. Lee, H. H. Liu, G. S. Ibbott, and R. Mohan, Reference photon dosimetry data and reference phase space data for the $6 \mathrm{MV}$ 
photon beam from Varian Clinac 2100 series linear accelerators, Med. Phys. $32,137-148$ (2005). (page 10)

[44] W. Schneider, T. Bortfeld, and W. Schlegel, Correlation between CT numbers and tissue parameters needed for Monte Carlo simulations of clinical dose distributions, Phys. Med. Biol. 45, 459 - 478 (2000). (pages 10, 18, 44, 50, 72. 77.

[45] J. V. Siebers, P. J. Keall, A. E. Nahum, and R. Mohan, Converting absorbed dose to medium to absorbed dose to water for Monte Carlo based photon beam dose calculation, Phys. Med. Biol. 45, 983 - 995 (2000). (pages 10, 18, 50, 77)

[46] H. Jiang and J. Seco and H. Paganetti, Effects of Hounsfield number conversion on CT based proton Monte Carlo dose calculations, Med. Phys. 34, 1439 - 1449 (2007). (page 10)

[47] H. Paganetti, Dose to water versus dose to medium in proton beam therapy, Phys. Med. Biol. 54, 4399 - 4421 (2009). (pages 10, 50, 77)

[48] F. Verhaegen and S. Devic, Sensitivity study for CT image use in Monte Carlo treatment planning, Phys. Med. Biol. 50, 937 - 946 (2005). (pages 10, 18, 102)

[49] M. Bazalova, L. Beaulieu, S. Palefsky, and V. Verghaegen, Correction of CT artifacts and its influence on Monte Carlo dose calculations, Med. Phys. 34, 2119 - 2132 (2007). (pages 10, 46)

[50] C. Xu, F. Vergaegen, D. Laurendeau, S. A. Enger, and L. Beaulieu, An algorithm for efficient metal artifact reductions in permanent seed implants, Med. Phys. 38, 47 - 56 (2011). (pages 10, 44, 68)

[51] Y. Takahashi, S. Mori, T. Kozuka, K. Gomi, T. Nose, T. Tahara, M. Oguchi, and T. Yamashita, Preliminary study of correction of metal artifacts due to I-125 seeds in postimplant dosimetry for prostate permanent implant brachytherapy, Radiat. Med. 24, 133 - 138 (2006). (pages 10, 44)

[52] P. S. Basran, A. Robertson, and D. Wells, CT image artifacts from brachytherapy seed implants: A postprocessing 3D adaptive median filter, Med. Phys. 38, 712 - 718 (2011). (pages 10, 44, 68, 72, 74, 81)

[53] H. Afsharpour, G. Landry, B. Reniers, J. P. Pignol, L. Beaulieu, and F. Verhaegen, Tissue modeling schemes in low energy breast brachytherapy, Phys. Med. Biol. 56, 7045 - 7060 (2011). (page 10)

[54] M. J. Rivard et.al., Comparison of dose calculation methods for brachytherapy of intraocular tumors, Med. Phys. 38, 306 - 316 (2011). (page 10)

[55] Y. Yang and M. J. Rivard, Evaluation of brachytherapy lung implant dose distributions from photon-emitting sources due to tissue heterogeneities, Med. Phys. 38, 5857 - 5862 (2011). (pages 10, 43, 117, 140, 141) 
[56] I. Kawrakow and D. W. O. Rogers, The EGSnrc Code System: Monte Carlo simulation of electron and photon transport, Technical Report PIRS-701, National Research Council of Canada, Ottawa, Canada, 2000. (page 13)

[57] I. Kawrakow, Accurate condensed history Monte Carlo simulation of electron transport. I. EGSnrc, the new EGS4 version, Med. Phys. 27, 485 - 498 (2000). (page 13)

[58] W. R. Nelson, H. Hirayama, and D. W. O. Rogers, The EGS4 code system, Report SLAC-265, Stanford Linear Accelerator Center, Stanford, California, 1985. (page 13)

[59] F. H. Attix, Introduction to Radiological Physics and Radiation Dosimetry, Wiley, New York, 1986. (page 13)

[60] E. B. Podgorsak, Radiation physics for medical physicists, Springer, Berlin, Heidelberg, New York, 2005. (page 13)

[61] H. E. Johns and J. R. Cunningham, The physics of radiology, 4th ed., Charles C. Thomas, Springfield, Illinois, 1983. (page 13)

[62] M. J. Berger, J. H. Hubbell, S. M. Seltzer, J. Chang, J. S. Coursey, R. Sukumar, D. S. Zucker, and K. Olsen, XCOM: Photon cross section database (version 1.5), Technical report, NIST, Gaithersburg, MD, http://physics.nist.gov/xcom, 2010. (page 13)

[63] M. J. Berger, Monte Carlo Calculation of the penetration and diffusion of fast charged particles, in Methods in Comput. Phys., edited by B. Alder, S. Fernbach, and M. Rotenberg, volume 1, pages 135 - 215, Academic, New York, 1963. (page 13)

[64] I. Kawrakow and A. F. Bielajew, On the condensed history technique for electron transport, Nuclear Instruments and Methods 142B, 253 - 280 (1998). (page 13)

[65] I. Kawrakow, Electron impact ionization cross sections for EGSnrc, Med. Phys. (abstract) 29, 1230 (2002). (page 14)

[66] R. E. P. Taylor, G. Yegin, and D. W. O. Rogers, Benchmarking BrachyDose: voxel-based EGSnrc Monte Carlo calculations of TG-43 dosimetry parameters, Med. Phys. 34, 445 - 457 (2007). (pages 14, 15, 16, 21)

[67] R. M. Thomson, G. Yegin, R. E. P. Taylor, J. G. H. Sutherland, and D. W. O. Rogers, Fast Monte Carlo dose calculations for brachytherapy with BrachyDose, Med. Phys. 37, (abstract) 3910 - 3911 (2010). (page 14)

[68] C. Thomason, T. Mackie, M. Lindstrom, and P. Higgins, The dose distribution surrounding Ir-192 and Cs-137 seed sources, Phys. Med. Biol. 36, 475 - 493 (1991). (page 14) 
[69] G. Luxton, Comparison of radiation-dosimetry in water and in solid phantom materials for I-125 and Pd-103 brachytherapy sources - EGS4 Monte Carlo study, Med. Phys. 21, 621 - 641 (1994). (page 14)

[70] R. Wang and R. S. Sloboda, EGS4 dosimetry calculations for cylindrically symmetric brachytherapy sources, Med. Phys. 23, 1459 - 1465 (1996). (page 14)

[71] E. Mainegra, R. Capote, and E. Lopez, Dose rate constants for ${ }^{103} \mathrm{Pd},{ }^{125} \mathrm{I}$, ${ }^{196} \mathrm{Yb},{ }^{192} \mathrm{Ir}$, brachytherapy sources: an EGS4 Monte Carlo Study, Phys. Med. Biol. 43, 1557 - 1566 (1998). (page 14)

[72] G. Luxton and G. Jozsef, Radial dose distribution, dose to water and dose rate constant for monoenergetic photon point sources from $10 \mathrm{keV}$ to $2 \mathrm{MeV}$ : EGS4 Monte Carlo model calculation, Med. Phys. 26, 2531 - 2538 (1999). (page 14)

[73] E. Mainegra, R. Capote, and E. Lopez, Radial dose functions for ${ }^{103} \mathrm{Pd},{ }^{125} \mathrm{I}$, ${ }^{196} \mathrm{Yb},{ }^{192} \mathrm{Ir}$, brachytherapy sources: an EGS4 Monte Carlo Study, Phys. Med. Biol. 45, 703 - 717 (2000). (page 14)

[74] R. E. Wallace, Empirical dosimetric characterization of model I125-SL ${ }^{125}$ Iodine brachytherapy source in phantom, Med. Phys. 27, 2796 - 2802 (2000). (page 14)

[75] R. Capote, E. Mainegra, and E. Lopez, Anisotropy functions for low energy interstitial brachytherapy sources: an EGS4 Monte Carlo Study, Phys. Med. Biol. 46, 135 - 150 (2001). (page 14)

[76] G. Yegin, A new approach to geometry modelling of Monte Carlo particle transport: an application to EGS, Nucl. Inst. Meth. B 211, 331 - 338 (2003). (pages 14, 15)

[77] R. E. P. Taylor and D. W. O. Rogers, An EGSnrc Monte Carlo-calculated database of TG-43 parameters, Med. Phys. 35, 4228 - 4241 (2008). (pages 15. 21, 23, 45, 98,

[78] R. E. P. Taylor and D. W. O. Rogers, EGSnrc Monte Carlo calculated dosimetry parameters for ${ }^{192} \mathrm{Ir}$ and ${ }^{169} \mathrm{Yb}$ brachytherapy sources, Med. Phys. 35, $4933-$ 4944 (2008). (page 15)

[79] M. Rodriguez and D. W. O. Rogers, On determining dose rate constants spectroscopically, Med. Phys. 40, 011713 (10pp) (2013). (page 15)

[80] J. H. Hubbell, Photon Mass Attenuation and Energy-absorption Coefficients from $1 \mathrm{keV}$ to $20 \mathrm{MeV}$, Int. J. Appl. Radiat. Isot. 33, 1269 - 1290 (1982). (page 16)

[81] R. P. Parker, P. A. Hobday, and K. J. Cassell, The direct use of CT numbers in radiotherapy dosage calculations for inhomogeneous media, Phys. Med. Biol. 24, 802 - 809 (1979). (page 18)

[82] I. Kawrakow, M. Fippel, and K. Friedrich, 3D Electron Dose Calculation using a Voxel based Monte Carlo Algorithm (VMC), Med. Phys. 23, 445 - 457 (1996). (page 18) 
[83] R. T. Mull, Mass estimates by computed tomography: physical density from CT numbers, American journal of roentgenology 143, 1101 - 1104 (1984). (page 18)

[84] D. Liu, M. Ajlouni, J. Jin, S. Ryu, F. Siddiqui, A. Patel, B. Movsas, and I. J. Chetty, Analysis of outcomes in radiation oncology: An integrated computational platform, Med. Phys. 36, 1680-1689 (2009). (page 18)

[85] H. Q. Woodard and D. R. White, The composition of body tissues, Brit. J. Radiol. 59, 1209 - 1219 (1986). (pages 18, 19, 24, 50, 75, 97, 119)

[86] D. R. White, H. Q. Woodard, and S. M. Hammond, Average soft-tissue and bone models for use in radiation dosimetry, Brit. J. Radiol. 60, 907 - 913 (1987). (pages 18, 19, 50)

[87] ICRU, Photon, Electron, Photon and Neutron Interaction Data for Body Tissues, ICRU Report 46, ICRU, Washington D.C., 1992. (pages 18, 19, 24, 50)

[88] National Electrical Manufacturers Association, DICOM: Digital Imaging and Communications in Medicine, 2013. (page 18)

[89] M. J. Yaffe and J. M. Boone et al., The myth of the 50-50 breast, Med. Phys. 36, 5437 - 5443 (2009). (pages 22, 23, 24, 25, 40)

[90] G. R. Hammerstein, D. W. Miller, D. R. White, M. E. Masterson, H. Q. Woodard, and J. S. Laughlin, Absorbed Radiation Dose in Mammography, Radiology 130, 485 - 491 (1979). (pages 22, 23, 25, 37)

[91] D. R. Dance, The Monte Carlo calculation of integral radiation dose in xeromammogrpahy, Phys. Med. Biol. 25, 25-37 (1980). (pages 22, 23, 25)

[92] I. Sechopoulos, A. Karellas, S. Suryanarayanan, S. Vedantham, and C. D'Orsi, Computation of the glandular radiation dose in digital tomosynthesis of the breast, Med. Phys. 34, 221 - 232 (2007). (pages 22, 23)

[93] R. C. Chen, R. Longo, L. Rigon, F. Zanconati, A. D. Pellegrin, F. Arfelli, D. Dreossi, R.-H. Menk, E. Vallazza, T. Q. Xiao, and E. Castelli, Measurement of the linear attenuation coefficients of breast tissues by synchrotron radiation computed tomography, Phys. Med. Biol. 55, 4993 - 5005 (2010). (page 22)

[94] P. C. Johns and M. J. Yaffe, X-ray characterisation of normal and neoplastic breast tissues, Phys. Med. Biol. 32, 675 - 696 (1987). (page 22)

[95] G. Landry et.al., Sensitivity of low energy brachytherapy Monte Carlo dose calculations to uncertainties in human tissue composition., Med. Phys. 37, $5188-5198$ (2010). (page 22)

[96] H. Afsharpour, J.-P. Pignol, B. Keller, J.-F. Carrier, B. Reniers, F. Verhaegen, and L. Beaulieu, Toward a more accurate dose calculation technique using a semiautomatic organ contouring in Monte Carlo postimplant assessment of breast LDR brachytherapy, Abstract book of 
MCTP2009: Second European Workshop on Monte Carlo Treatment Planning, available at http://www.mctp2009.org/images/documents/EGWMCTP\%20Workshop\%202009\%20Book\%20of\%20Abstracts.pdf ， 98 - 101 (2009). (pages 22, 30)

[97] M. Bazalova and E. E. Graves, The importance of tissue segmentation for dose calculations for kilovoltage radiation therapy, Med. Phys. 38, 3039 - 3049 (2011). (page 22)

[98] R. M. Thomson and D. W. O. Rogers, Monte Carlo dosimetry for ${ }^{125} \mathrm{I}$ and ${ }^{103} \mathrm{Pd}$ eye plaque brachytherapy with various seed models, Med. Phys. 37, 368 - 376 (2010). (page 23)

[99] J.-P. Pignol, E. Rakovitch, B. M. Keller, R. Sankreacha, and C. Chartier, Tolerance and Acceptance Results of a Palladium-103 Permanent Breast Seed Implant Phase I/II Study, Int. J. Radiat. Oncol. Biol. Phys. 73, 1482 - 1488 (2009). (page 24)

[100] E. Zastrow, S. K. Davis, M. Lazebnik, F. Kelcz, B. D. V. Veen, and S. C. Hagness, Development of Anatomically Realistic Numerical Breast Phantoms With Accurate Dielectric Properties for Modeling Microwave Interactions With the Human Breast, IEEE Transactions on Biomedical Engineering 55, 2792 2800 (2010). (pages 27, 36)

[101] O. Hassler, Microradiographic investigations of calcifications of the female breast, Cancer 23, 1103-1109 (1969). (page 28)

[102] D. M. Hansell, J. C. Cooke, C. A. Parsons, S. H. Evans, D. R. Dance, J. M. Bliss, and I. Ilesley, A quantitative analysis of the spatial relationships of grouped microcalcifications demonstrated on xeromammography in benign and malignant breast disease, Brit. J. Radiol. 61, 21-25 (1988). (page 28)

[103] M. Abdoli, M. R. Ay, A. Ahmadian, and H. Zaidi, A virtual sinogram method to reduce dental metallic implant artefacts in computed tomography-based attenuation correction for PET, Nuclear Medicine Communications 31, 22 - 31 (2010). (page 46)

[104] M. Abdoli, M. R. Ay, A. Ahmadian, R. A. J. O. Dierckx, and H. Zaidi, Reduction of dental filling metallic artifacts in CT-based attenuation correction of PET data using weighted virtual sinograms optimized by a genetic algorithm, Med. Phys. 37, 6166 - 6177 (2010). (page 46)

[105] W. A. Kalender, R. Hebel, and J. Ebersberger, Reduction of CT artifacts caused by metallic implants, Radiology 164, 576 - 577 (1987). (page 46)

[106] J. C. Roeske, C. Lund, C. A. Pelizzari, X. Pan, and A. J. Mundt, Reduction of computed tomography metal artifacts due to the Fletcher-Suit applicator in gynecology patients receiving intracavitary brachytherapy, Brachytherapy 2, $207-214$ (2003). (page 46) 
[107] M. Yazdi, L. Gingras, and L. Beaulieu, An adaptive approach to metal artifact reduction in helical computed tomography for radiation therapy treatment planning: Experimental and clinical studies, Int. J. Radiat. Oncol. Biol. Phys. 62, $1224-1231$ (2005). (page 46)

[108] G. Landry, P. V. Granton, B. Reniers, M. C. Ollers, L. Beaulieu, J. E. Wilderger, and F. Vergaegen, Simulation study on potential accuracy gains from dual energy CT tissue segmentation for low-energy brachytherapy Monte Carlo dose calculations, Phys. Med. Biol. 56, 6257 - 6278 (2011). (pages 69, 102)

[109] K. M. Furutani, R. C. Miller, L. B. McLemore, C. C. Goulet, D. H. Brinkmann, and M. G. Haddock, 4D CT dosimetric analysis of the Mayo Clinic brachytherapy technique for a cholangiocarcinoma patient, Brachytherapy 5, 84 (2006). (page 70)

[110] R. O. Ottosson and C. F. Behrens, CTC-ask: a new algorithm for conversion of CT numbers to tissue parameters for Monte Carlo dose calculations applying DICOM RS knowledge, Phys. Med. Biol. 56, N263-N274 (2011). (page 73)

[111] J. G. H. Sutherland, K. M. Furutani, Y. I. Garces, and R. M. Thomson, Modelbased dose calculations for ${ }^{125}$ I lung brachytherapy, Med. Phys. 39, $4365-4377$ (2012). (pages 89, 93, 100)

[112] M. J. Rivard, Brachytherapy dosimetry parameters calculated for a ${ }^{131} \mathrm{Cs}$ source, Med. Phys. 34, 754 - 762 (2007). (page 97)

[113] Brookhaven National Laboratory, National Nuclear Data Center, http://www.nndc.bnl.gov/nudat2. (page 97)

[114] S. Thomas, A. Colonias, H. Odau, R. K. Das, S. M. Bentzen, R. R. Patel, and D. Khuntia, Feasibility of Cs-131 for intraoperative brachytherapy for patients undergoing sublobar resections for early stage non-small cell lung cancer (NSCLC): A dosimetric comparison with I-125 and Cs-131, Brachytherapy 106, 108 (2007). (page 117)

[115] D. Nori, X. Li, and T. Pugkhem, Intraoperative brachytherapy using gelfoam radioactive plaque implants for resected stage III non-small cell lung cancer with positive margin: A pilot study, Journal of surgical oncology 60, 257-261 (1995). (page 117)

[116] S. A. Enger, D. R. Fisher, and R. T. Flynn, Gadolinium-153 as a brachytherapy isotope, Phys. Med. Biol. 58, 957 - 964 (2013). (page 117)

[117] M. G. Trombetta, A. Colonias, D. Makishi, R. Keenan, E. D. Werts, R. Landreneau, and D. S. Parda, Tolerance of the aorta using intraoperative iodine- 125 interstitial brachytherapy in cancer of the lung, Brachytherapy 7, $50-54$ (2008). (pages 118, 136)

[118] L. B. Marks et al, Radiation dose-volume effects in the lung, Int. J. Radiat. Oncol. Biol. Phys. 76, S70 - S76 (2010). (page 134) 
[119] J. L. W. S. A. Mccormick, B. Sood, A. Alieri, and B. Vikram, Tolerance of the carotid sheath contents to brachytherapy: An experimental study, The Laryngoscope 101, 587 - 591 (1991). (page 136)

[120] S. C. Darby et al., Radiation-related heart disease: current knowledge and future prospects, Int. J. Radiat. Oncol. Biol. Phys. 76 (2010). (page 138)

[121] S. C. Darby et al., Risk of Ischemic Heart Disease in Women after Radiotherapy for Breast Cancer, New England Journal of Medicine 368, 987-998 (2013). (page 138) 